\title{
Spanning connectedness and Hamiltonian thickness of graphs and interval graphs*
}

\author{
Peng $\mathrm{Li}^{1,2}$ \\ Yaokun $\mathrm{Wu}^{1} \|^{\dagger}$ \\ 1 Department of Mathematics, and MOE-LSC, Shanghai Jiao Tong University, China \\ 2 School of Mathematics and Statistics, Chongqing University of Technology, China
}

received $28^{\text {th }}$ Nov. 2013, revised $15^{\text {th }}$ Jan. 2015, accepted $9^{\text {th }}$ Nov. 2015.

\begin{abstract}
A spanning connectedness property is one which involves the robust existence of a spanning subgraph which is of some special form, say a Hamiltonian cycle in which a sequence of vertices appear in an arbitrarily given ordering, or a Hamiltonian path in the subgraph obtained by deleting any three vertices, or three internally-vertex-disjoint paths with any given endpoints such that the three paths meet every vertex of the graph and cover the edges of an almost arbitrarily given linear forest of a certain fixed size. Let $\pi=\pi_{1} \cdots \pi_{n}$ be an ordering of the vertices of an $n$-vertex graph $G$. For any positive integer $k \leq n-1$, we call $\pi$ a $k$-thick Hamiltonian vertex ordering of $G$ provided it holds for all $i \in\{1, \ldots, n-1\}$ that $\pi_{i} \pi_{i+1} \in E(G)$ and the number of neighbors of $\pi_{i}$ among $\left\{\pi_{i+1}, \ldots, \pi_{n}\right\}$ is at least $\min \{n-i, k\}$; For any nonnegative integer $k$, we say that $\pi$ is a $-k$-thick Hamiltonian vertex ordering of $G$ provided $\left|\left\{i: \pi_{i} \pi_{i+1} \notin E(G), 1 \leq i \leq n-1\right\}\right| \leq k+1$. Our main discovery is that the existence of a thick Hamiltonian vertex ordering will guarantee that the graph has various kinds of spanning connectedness properties and that for interval graphs, quite a few seemingly unrelated spanning connectedness properties are equivalent to the existence of a thick Hamiltonian vertex ordering. Due to the connection between Hamiltonian thickness and spanning connectedness properties, we can present several linear time algorithms for associated problems. This paper suggests that much work in graph theory may have a spanning version which deserves further study, and that the Hamiltonian thickness may be a useful concept in understanding many spanning connectedness properties.
\end{abstract}

Keywords: Chvátal-Erdős Theorem, fault tolerance, forward degree sequence, graph power, interval representation, local spanning cut function, normal path algorithm, path cover number, rooted path system, scattering number, spanning rail connectivity, spanning fan connectivity.

\footnotetext{
*This work was supported by the National Natural Science Foundation of China (No. 11271255) and the Chinese Ministry of Education (No. 108056).

$\dagger$ Corresponding author.
}

1365-8050 @ 2015 Discrete Mathematics and Theoretical Computer Science (DMTCS), Nancy, France 


\section{Contents}

$\begin{array}{lll}1 & \text { Introduction } & 127\end{array}$

1.1 Background . . . . . . . . . . . . . . . . . . . . . . . . . . . . . . . . 127

1.2 Interval graphs and spanning connectedness $\ldots \ldots \ldots \ldots \ldots \ldots \ldots$

1.3 Hamiltonian thickness $\ldots \ldots \ldots \ldots$. . . . . . . . . . . . . . . . 130

1.4 Our work $\ldots \ldots \ldots \ldots \ldots$. . . . . . . . . . . . . . . . . . . . . . 132

2 Spanning connectedness properties 132

2.1 Endpoint constraints . . . . . . . . . . . . . . . . . . . . . . . 133

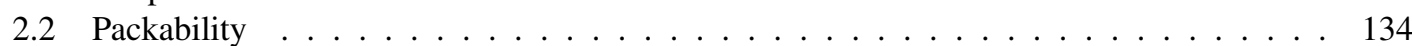

2.3 Ordering constraints . . . . . . . . . . . . . . . . . . . . . . . . . . . 134

2.4 Extension property . . . . . . . . . . . . . . . . . . . . . . . . . . . 135

2.5 Fault-tolerance $\ldots \ldots \ldots \ldots \ldots \ldots$. . . . . . . . . . . . . . . . . . . . 136

2.6 Passing through specified edges with fault-tolerance . . . . . . . . . . . . . . . . 136

$\begin{array}{lll}3 \text { Main results } & 137\end{array}$

3.1 General graphs . . . . . . . . . . . . . . . . . . . . . . . . . 137

3.1.1 Spanning connectedness properties . . . . . . . . . . . . . . . . . 137

$3.1 .2 \quad$ Scattering number and Hamiltonian thickness $\ldots \ldots \ldots$. . . . . . . . . . . . 139

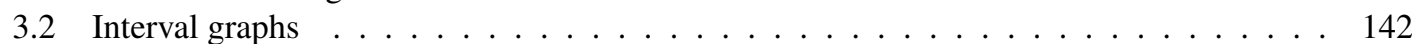

$3.2 .1 \quad$ Normal path algorithm . . . . . . . . . . . . . . . . . . . . . . . . . 142

3.2 .2 Characterizations of $\digamma^{k} \ldots \ldots \ldots \ldots \ldots \ldots$

3.2 .3 Ordering constraints . . . . . . . . . . . . . . . . . . . . . . 146

3.2.4 Equivalences among various connectedness parameters . . . . . . . . . . . . . . . 147

3.2 .5 Extension properties $\ldots \ldots \ldots \ldots \ldots$. . . . . . . . . . . . . . . . . . 148

$3.2 .6 \quad$ Several linear time algorithms . . . . . . . . . . . . . . . . . . . . . . . . . . . 149

3.2.7 Spanning version of the Chvátal-Erdős Theorem and the Menger's Theorem. . . . 151

3.2 .8 Graph power and traceable number $\ldots \ldots \ldots \ldots \ldots \ldots$

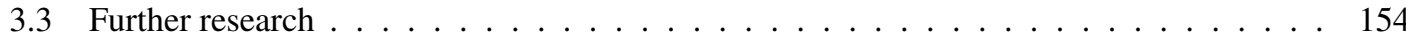

$\begin{array}{lll}4 & \text { Proofs } & 158\end{array}$

4.1 General graphs . . . . . . . . . . . . . . . . . . . . . . . . 158

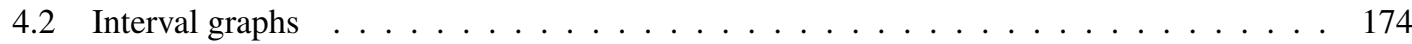

\begin{tabular}{ll}
\hline Bibliography & 203
\end{tabular} 


\section{Introduction}

\subsection{Background}

A subgraph of a graph is called a spanning subgraph if it contains all the vertices of that graph. Spanning paths and spanning cycles are widely known as Hamiltonian paths and Hamiltonian cycles, respectively. They are named in honour of Sir William Rowan Hamilton in view of the fact that Hamilton invented the Icosian Game, a game that challenges the players with various mathematical puzzles that relate to spanning subgraphs of the dodecahedral graph. E.g., as reported in Barnett (6), Cook (25), using his Icosian calculus, probably the earliest contribution to what later was to be dubbed Combinatorial Group Theory, Hamilton could show that, no matter what (simple) path of length 4 in the dodecahedral graph gets chosen as a starting path, one can always complete that path to a cyclic tour through all the vertices a fact that he noted already in a letter to his friend John T. Graves on October 17, 1856. That is, Hamilton showed not only the existence of a specific pattern of connectedness, namely that of a spanning cycle, but also the fact that this pattern is robust in the sense that any (simple) path of length four in that graph can be extended to such a subgraph. The existence of Hamiltonian cycles/paths has become one of the most studied areas of graph theory since then; See Bondy and Murty (9).

Although Hamilton himself was already not just concerned with the existence of a Hamiltonian cycle/path, it is only a relatively recent trend to systematically study various forms and degrees of connectedness of graphs in terms of the existence of certain types of connected spanning subgraphs under various kinds of robustness requirements - an endeavor that may actually be quite useful for various applications, e.g., for providing combinatorial Gray codes as discussed in Savage (99), as supplying a spanning structure yields in general a good way of displaying/generating/connecting all objects under consideration in a more or less systematic way.

Here, we will concentrate our efforts on rooted path systems, that is, certain natural generalizations of paths and cycles. To this end, we write $[k]$ for the set $\{1, \ldots, k\}$ for any positive integer $k$ and we assume that $G=(V(G), E(G))$ is a graph with vertex set $V(G)$ and edge set $E(G) \subseteq\left(\begin{array}{c}V(G) \\ 2\end{array}\right)$. A path $P$ in $G$ - i.e., a finite sequence $x_{0}, \ldots, x_{\ell}$ of pairwise distinct vertices of $G$ with $x_{i-1} x_{i} \in E(G)$ for all $i \in[\ell]-$ will also be called an $x_{0}, x_{\ell}$-path (of length $\ell>0$ ) and denoted by $P=\left(x_{0}, \ldots, x_{\ell}\right)$, the vertices $x_{0}$ and $x_{\ell}$ will be called the endpoints of this path $P$, and the set $\left\{x_{0}, \ldots, x_{\ell}\right\}$ of vertices visited by this path $P$ will be denoted by $V(P)$. For any vertex $v \in V(G)$, we will think of $v$ itself as a path in $G$ of length 0 and with a single endpoint, namely $v$ itself. For a positive integer $k$, if $\left(x_{0}, x_{1}, \ldots, x_{k+1}\right)$ is a path and $x_{0} x_{k+1}$ belongs to $E(G)$, then we say that the cyclic sequence $x_{0}, x_{1}, \ldots, x_{k+1}$ forms a cycle $C$ of length $k+2$; We adopt the notation $\left\langle x_{0}, x_{1}, \ldots, x_{k+1}\right\rangle$ for this cycle and write $V(C)$ for the set of vertices on $C$. A Hamiltonian cycle of a graph is a spanning cycle in it, i.e., a cycle through every vertex, and a Hamiltonian path is a spanning path. We refer to any cycle passing through a vertex $x$ as an $x, x$-path. For any family $R=R_{k}$ consisting of $k$ pairs of vertices $\left(s_{1}, t_{1}\right), \ldots,\left(s_{k}, t_{k}\right) \in V(G) \times V(G)$, a path system of $G$ rooted at $R$ is a family $P_{1}, \ldots, P_{k}$ of $k$ paths/cycles such that $P_{i}$ is an $s_{i}, t_{i}$-path for each $i \in[k]$ and $V\left(P_{i}\right) \cap V\left(P_{j}\right)=\left\{s_{i}, t_{i}\right\} \cap\left\{s_{j}, t_{j}\right\}$ holds for every two distinct indices $i, j \in[k]$. In contrast to path systems as considered in the context of some classical graph connectivity theorems, say Menger's Theorem, we are not merely asking for disjoint paths between two vertex sets: We specify a pairing of these endpoints and view the family consisting of these endpoint pairs as the root of the path system.

If a graph has at least $2 k$ vertices and has a path system rooted at $R_{k}$ for every family $R_{k}=\left(\left(s_{i}, t_{i}\right)\right)_{i \in[k]}$ of $k$ vertex pairs with $\left\{s_{i}, t_{i}: i \in[k]\right\}$ being a $2 k$-element subset of the graph vertex set, the graph is 
said to be $k$-linked; The property of being $k$-linked was addressed by Larman and Mani (70), Mader (79), Robertson and Seymour (95). If $\left\{s, t_{1}, \ldots, t_{k}\right\}$ is a set of $k+1$ distinct vertices, any path system rooted at $R=\left(\left(s, t_{i}\right)\right)_{i \in[k]}$ is called an $(s ; U)$-fan, or simply a $k$-fan, where $U:=\left\{t_{1}, \ldots, t_{k}\right\}$; Lin et al. (78) established some results about $k$-fans. When the family $R$ consists of the fixed pair $(s, t)$ with multiplicity $k$ and $s \neq t$, a path system rooted at $R$ is called a $k$-rail between $s$ and $t$ in Sørensen and Thomassen (102) while a spanning $k$-rail is termed a $k$-container in Hsu (49) and a spanning $k$-stave in Broersma et al. (11). A Hamiltonian path $P$ is nothing but a spanning 1-fan (1-rail) rooted at the endpoints of $P$; Forgetting its root, a spanning 2-rail yields just a Hamiltonian cycle. When $\left\{s_{1}, \ldots, s_{k}, t_{1}, \ldots, t_{k}\right\}$ has cardinality $2 k$, a spanning path system rooted at $R=\left(\left(s_{i}, t_{i}\right)\right)_{i \in[k]}$ is a path cover of $G$ of size $k$, namely a set of $k$ paths such that every vertex $v \in V(G)$ belongs to exactly one path; See Arikati and Pandu Rangan (3) for some related work. The " $k$-DPC problem" is the problem of characterizing those graphs in which there exists, for any family $R=\left(\left(s_{i}, t_{i}\right)\right)_{i \in[k]}$ of $k$ vertex pairs with $\left\{s_{1}, \ldots, s_{k}, t_{1}, \ldots, t_{k}\right\}=2 k$, a spanning path system rooted at $R$. It originated from the study of interconnection networks by Jo et al. (59), Park et al. (91) and can be viewed as a "spanning version" of the problem of checking the property of being $k$-linked.

In line with the Icosian paradigm, we care about not only the mere existence of connected spanning subgraphs that belong to certain isomorphisms classes of graphs, but also to which extent the existence of such subgraphs is rich and robust and can hold various pieces of the graph tightly with flexibility. Correspondingly, we shall propose a bunch of measures/properties that reflect various forms and degrees of connectedness of graphs in such terms. We postpone a detailed description of these requirements to $\$ 2$.

\subsection{Interval graphs and spanning connectedness}

An interval representation $\mathcal{I}$ of a graph $G$ associates, to every $v \in V(G)$, a nonempty interval $\mathcal{I}(v)=$ $\left[\ell_{\mathcal{I}}(v), r_{\mathcal{I}}(v)\right]$ on the real line such that, for all $u, v \in V(G)$, one has $u v \in E(G)$ if and only if $u \neq v$ and $\mathcal{I}(u) \cap \mathcal{I}(v) \neq \emptyset$ holds. If $\mathcal{I}$ is an interval representation of a graph $G$ such that the length of the intervals $\mathcal{I}(v)$ is a constant value for $v \in V(G)$, then we say that $\mathcal{I}$ is a unit interval representation. A graph for which an interval representation exists is called an interval graph and a graph having a unit interval representation is a unit interval graph. The combinatorics of interval graphs has both rich mathematical theory and important practical applications. Due to their simple geometric representation, many problems regarding the path and/or cycle structure of a graph can be solved much more easily for interval graphs than for arbitrary graphs. Nonetheless, there are still many interesting open problems and conjectures relating to interval graphs or even unit interval graphs.

A cocomparability graph is a graph for which one can define a partial order " $\preceq$ " on its vertex set such that, for all $u, v \in V(G)$, one has $u v \in E(G)$ if and only if neither $u \preceq v$ nor $v \preceq u$ holds. For any graph $G$ and any $v \in V(G)$, the open neighborhood of $v$ in $G$ is the set $\{w \in V(G): v w \in E(G)\}$, which is denoted by $N_{G}(v)$, while the closed neighborhood of $v$ in $G$ is $\{v\} \cup N_{G}(v)$ and denoted by $N_{G}[v]$. A simplicial vertex in a graph $G$ is a vertex such that $N_{G}(v)$ (or, equivalently, $N_{G}[v]$ ) is a clique in $G$. A graph $G$ with $n$ vertices is chordal provided it has a vertex ordering $\pi_{1} \cdots \pi_{n}$ such that $\pi_{i}$ is simplicial in $G\left[\pi_{1}, \ldots, \pi_{i}\right]$ for every $i \in[n]$. Equivalently, we define a graph $G$ to be chordal if every chord-free cycle in $G$ (if any) has exactly three vertices. Three vertices of a graph form an asteroidal triple if every two of them are connected by a path avoiding the neighbourhood of the third one. Accordingly, a graph without asteroidal triples is called AT-free. Recall that a graph is an interval graph if and only if it is both chordal and AT-free, if and only if it is both a chordal graph and a cocomparability graph, and if and only 
if it is a cocomparability graph which does not contain a 4-cycle as an induced subgraph; Many properties of these graph classes can be found in Golumbic (40). The bulk of this paper is devoted to a systematic discussion of various connectedness properties of interval graphs and some related graph classes.

Intuitively, a dense graph should be "highly connected" in whichever way this might be defined. Our main interest here is to design sparse (interval) graphs that exhibit a high degree of connectedness in terms of connected spanning subgraphs, to expose some structural aspects of the (interval) graphs that enforce a high degree of connectedness (as measured in these terms), and to elucidate the connection between various possible spanning connectedness properties of (interval) graphs.

Various parameters for measuring the "spanning connectedness" of graphs, especially their vulnerability, have been proposed in the literature. For any nonnegative integer $k$, a graph $G$ is called $k$-connected if it has at least $k+1$ vertices and the removal of no $S \in\left(\begin{array}{c}V(G) \\ k-1\end{array}\right)$ renders $G$ disconnected. The vertex connectivity $\kappa(G)$ of a graph $G$ is the maximum value of $k$ for which $G$ is $k$-connected. We call $S \subseteq V(G)$ a minimum vertex separator of $G$ provided $G-S$ is disconnected and $|S|=\kappa(G)$. For any graph $G$, let c $(G)$ denote the number of connected components of $G$. For any subset $S$ of $V(G)$, denote by $G[S]:=\left(S, V(G) \cap\left(\begin{array}{c}S \\ 2\end{array}\right)\right)$ the induced subgraph of $G$ with vertex set $S$ and put $G-S:=G[V(G) \backslash S]$. For each $v \in V(G)$, we often use $G-v$ as a shorthand for $G-\{v\}$ and call it a vertex-deleted subgraph of $G$. The toughness of a graph $G$, which was first considered by Chvátal (23), is

$$
\mathrm{t}(G):=\min \left\{\frac{|S|}{\mathrm{c}(G-S)}: S \subseteq V(G), \mathrm{c}(G-S)>1\right\}
$$

if $G$ is not a complete graph and

$$
\mathrm{t}(G):=\infty
$$

if $G$ is a complete graph, while a toughness determining set of $G$ is any subset $S$ of $V(G)$ with $\mathrm{t}(G)=$ $\frac{|S|}{\mathrm{c}(G-S)}$. Further, a graph $G$ is called $t$-tough for any real number $t$ satisfying $0 \leq t \leq \mathrm{t}(G)$. As an additive dual of the toughness parameter, the scattering number of a graph $G$, denoted by $\operatorname{sc}(G)$ and studied by Jamrozik et al. (58), Jung (60), is

$$
\max \{\mathrm{c}(G-S)-|S|: S \subseteq V(G), \mathrm{c}(G-S)>1\}
$$

if $G$ is not a complete graph, and is

$$
3-|V(G)|
$$

otherwise. Every subset $S$ of $V(G)$ satisfying $\operatorname{sc}(G)=\mathrm{c}(G-S)-|S|$ and $\mathrm{c}(G-S)>1$ is called a scattering set of $G$; See Broersma et al. (11). Excepting complete graphs, all graphs $G$ satisfy

$$
\operatorname{sc}(G) \geq 2-(|V(G)|-2)=4-|V(G)| .
$$

Broersma et al. (11) found that the scattering number determines quite a few seemingly unrelated connectedness properties of interval graphs. The path cover number of a graph $G$, denoted by $\pi(G)$, is the minimum integer $k$ such that $G$ has a path cover of size $k$. An easy observation of Shih et al. (101, Proposition 2.2) is that

$$
\operatorname{sc}(G) \leq \pi(G) .
$$

must hold for every graph $G$. 


\subsection{Hamiltonian thickness}

This subsection is to identify a graph parameter that we call "Hamiltonian thickness" which will allow us to present a unified approach for establishing various connectednesss properties of graphs and interval graphs. Hamilton was actually not the first person to study the spanning connectedness of graphs. Indeed, in 1759, Euler already solved the knight's tour problem on a chessboard of sixty-four squares Cook (25, Fig. 2.11) while Kirkman investigated Hamiltonian cycles in polyhedra one year before Hamilton. Yet, Hamilton's Icosian game exhibits many more aspects of connectedness, and so we try to honor him with the choice of the name Hamiltonian thickness.

The parameters vertex connectivity, scattering number and path cover number have minimum vertex separator, scattering set and minimal path cover, respectively, as the positive certifying structures. For the Hamiltonian thickness parameter, the positive certifying structure is some kind of vertex orderings. So, let us first of all introduce some concepts relating to such orderings. For any set $S$ and any nonnegative integer $k \leq|S|$, we denote by

$$
S^{k}:=\left\{\left(\pi_{1}, \ldots, \pi_{k}\right) \in S^{k}:\left|\left\{\pi_{1}, \ldots, \pi_{k}\right\}\right|=k\right\}
$$

the set of all sequences or words $\pi=\pi_{1} \pi_{2} \cdots \pi_{k}$ in $S^{k}$ without repetition, also known as the set of all partial $k$-permutations over $S$. If $S$ is finite and $k$ coincides with the cardinality $|S|$ of $S$, the set $S^{\underline{k}}$ is just the set of all permutations or orderings of $S$ that we also denote by $S !$. For any positive integer $k$, any word $\pi \in S^{k}$, and any two indices $p, q \in[k]$ with $1 \leq p \leq q \leq k$, we denote by $\pi[p, q]$ the word $\pi_{p} \cdots \pi_{q}$. And for any two words $\pi=\pi_{1} \pi_{2} \cdots \pi_{k} \in S^{k}$ and $\tau=\tau_{1} \tau_{2} \cdots \tau_{j} \in S^{j}$, we denote by $\pi \tau$ the word $\pi_{1} \cdots \pi_{k} \tau_{1} \cdots \tau_{j}$ of length $k+j$ obtained by concatenating the two words $\pi$ and $\tau$. Note that, given two sets $S$ and $T$ and two partial permutations $\pi \in S^{\underline{k}}$ and $\tau \in T^{j}$, their concatenation $\pi \tau \in(S \cup T)^{k+j}$ is actually a partial permutation of $S \cup T$ in case $S$ and $T$ are disjoint.

Next, given a graph $G$ on $n$ vertices, a permutation $\pi=\pi_{1} \cdots \pi_{n} \in V(G)$ ! and an index $i \in[n]$, we define the forward degree $\mathrm{d}_{G, \pi}(i)$ of the vertex $\pi_{i}$ with respect to the graph $G$ and the vertex ordering $\pi$ by putting

$$
\mathrm{d}_{G, \pi}(i):=\left|\left\{j: \pi_{j} \in N_{G}\left(\pi_{i}\right), i<j \leq n\right\}\right|,
$$

and we dubb the resulting sequence $\left(\mathrm{d}_{G, \pi}(1), \ldots, \mathrm{d}_{G, \pi}(n)\right)$ of all forward degrees of the vertices in $G$ the forward degree sequence of $G$ with respect to $\pi$. Further, given any $k \in[n-1]$, we say that $\pi$ is a $k$-thick ordering of $G$ provided $\mathrm{d}_{G, \pi}(i) \geq \min \{k, n-i\}$ holds for all $i \in[n]$, that is, if and only if the last $k+1$ vertices (relative to $\pi)\left\{\pi_{n-k}, \cdots, \pi_{n}\right\}$ form a clique and $\mathrm{d}_{G, \pi}(i) \geq k$ holds for all $i \in[n-k-1]$. An ordering $\pi=\left(\pi_{1}, \ldots, \pi_{n}\right) \in V(G)$ ! will be called a Hamiltonian vertex ordering of $G$ if the sequence $\left(\pi_{1}, \ldots, \pi_{n}\right)$ is a Hamiltonian path of $G$. For any positive integer $k$, a $k$-thick Hamiltonian vertex ordering is an ordering which is both $k$-thick and Hamiltonian. To simplify induction later on and to tackle algorithmic problems on general graphs, say finding a minimum path cover, we will also say that $\pi$ is a $-k$-thick Hamiltonian vertex ordering for some nonnegative integer $k$ provided $\left|\left\{i \in[n-1]: \pi_{i} \pi_{i+1} \notin E(G)\right\}\right| \leq k+1$ holds. For any $\pi \in V(G)$ !, the Hamiltonian thickness $\mathcal{H}_{G}(\pi)$ of $\pi$ with respect to $G$ is the maximum integer $k$ such that $\pi$ is a $k$ thick Hamiltonian vertex ordering of $G$. Note that $\mathcal{H}_{G}(\pi) \leq 0$ if and only if either $|V(G)|=n=1$ or $\mathcal{H}_{G}(\pi)=1-\left|\left\{i \in[n-1]: \pi_{i} \pi_{i+1} \notin E(G)\right\}\right|$. For every $v \in V(G)$, the height of $v$ in $G$, denoted by $\mathcal{H}_{G}(v)$, is defined by putting

$$
\mathcal{H}_{G}(v):=\max \left\{\mathcal{H}_{G}(\pi): \pi \text { is an ordering of } V(G) \text { starting at } v\right\} .
$$


For any integer $k$, denote by $\digamma^{k}$ the class of all graphs which possess a $k$-thick Hamiltonian vertex ordering. Note that $\digamma^{k} \supseteq \digamma^{\ell}$ holds for all integers $k, \ell$ with $k \leq \ell$. For every graph $G$, the thickness of $G$ is the largest integer $k$ such that $G$ has a $k$-thick ordering and the Hamiltonian thickness of $G$ is

$$
\mathcal{H}(G):=\max \left\{k: G \in \digamma^{k}\right\}=\max _{v \in V(G)} \mathcal{H}_{G}(v) .
$$

We write $K_{n}$ for the complete graph on $n$ vertices and $\overline{K_{n}}$ for the complement of $K_{n}$, namely the graph consisting of $n$ isolated vertices. Note that

$$
\mathcal{H}\left(\overline{K_{n}}\right)=\left\{\begin{aligned}
0, & \text { if } n=1 \\
2-n, & \text { else }
\end{aligned}\right.
$$

and

$$
\mathcal{H}\left(K_{n}\right)=n-1=\kappa\left(K_{n}\right)
$$

for every positive integer $n$. If $G$ has at least two vertices and $\mathcal{H}(G) \leq 1,2-\mathcal{H}(G)$ is the minimum size of a path cover of $G$, while $1-\mathcal{H}(G)$ coincides with the so-called Hamiltonian path completion number of $G$ as discussed by Damaschke et al. (30), namely the minimum number of additional edges whose addition will turn $G$ into a graph with a Hamiltonian path. We can write this observation simply as

$$
\pi(G)=\left\{\begin{aligned}
1, & \text { if } G=K_{1}, \\
\max \{1,2-\mathcal{H}(G)\}, & \text { else. }
\end{aligned}\right.
$$

If $G$ is not a complete graph, it is clear that $2-|V(G)| \leq \mathcal{H}(G) \leq|V(G)|-2$ holds. We call a vertex $v$ of $G$ universal provided $N_{G}[v]=V(G)$ holds. The Hamiltonian thickness of $G$ is not less than a nonpositive integer $k$ if and only if adding $1-k$ universal vertices to $G$ will cause the existence of a Hamiltonian path.

The concept of forward degree sequence already arises in the study of the chip firing game (divisors on graphs), say Baker and Norine (5), Remark 1.10, Lemma 3.2, Theorem 3.3), and in the study of the offer rejection problem, say Chen et al. (20). The concept of thick ordering also appears implicitly in the literature. Indeed, Bondy and Murty (9, Lemma 9.3) claims that a graph $G$ can have a $k$-thick ordering only if $k \leq \kappa(G)$ holds, that is,

$$
\mathcal{H}(G) \leq \kappa(G)
$$

must hold - this also follows both from Theorem 15 and from Theorem $22 \mathrm{~g}) \Rightarrow \mathrm{c}$ ) in this paper. It is worth mentioning that the thickness of a graph may be viewed as a concept that is somehow dual to the degeneracy parameter, which are studied in Chrobak and Eppstein (22), Erdős and Hajnal (34), Kirousis and Thilikos (64), Lick and White (75): The degeneracy of a graph $G$, denoted by $\operatorname{dgn}(G)$, is the least integer $k$ for which there exists an ordering of the vertices of $G$ in which each vertex has $k$ or fewer neighbors that occur earlier than it in the ordering. For each graph $G$, it is clear that $\operatorname{dgn}(G)$ is no smaller than the minimum degree of $G$ and hence

$$
\operatorname{dgn}(G) \geq \kappa(G)
$$

follows. If $G$ is an interval graph, Bondy and Murty (9, Exercise 14.5.10) and Woodall (106, §6.2) showed that a $\operatorname{dgn}(G)+1$ is equal to the clique number of $G$. 


\subsection{Our work}

Let us now summarize the main contribution of this paper. We find that a graph with Hamiltonian thickness $O(k)$ exhibits a high degree of connectedness in terms of rooted $k$-path systems $(\$ 3.1 .1)$ which will enable us to construct sparse graphs with good "spanning connectedness". We will discuss the interesting relationship between the scattering number of a graph and its Hamiltonian thickness as well as the behavior of the Hamiltonian thickness upon vertex deletion $(\$ 3.1 .2)$. For interval graphs, we will show that almost all spanning connectedness properties introduced in this section and in $\$ 2$ are equivalent and are characterized by the Hamiltonian thickness parameter $(33.2 .2,33.2 .3,33.2 .4$, 3.2 .5 . This includes the useful observation that, for interval graphs, Hamiltonian thickness gives the same information as the scattering number (Theorem 33). It is known that an interval graph is "cycle extendable" if and only if it is Hamiltonian; See Abueida and Srithran (1), Chen et al. (19). With the help of the equivalence of various spanning connectedness properties for interval graphs, this extension result about spanning 2-rails (Hamiltonian cycles) can be extended to general spanning $k$-rails (Theorem 46). Almost all proofs of our results are constructive and so correspond to some efficient algorithms. In 3.2.6, we demonstrate the usefulness of our work on Hamiltonian thickness in algorithm design. We develop a linear-time algorithm to determine the spanning rail connectivity of an interval graph, thus answering an open problem from Broersma et al. (11); We also get a linear-time algorithm to calculate a scattering set of an interval graph that is not complete - another linear-time algorithm for this task has recently been provided in Broersma et al. (11, Corollary 1). Using the concept of Hamiltonian thickness, we will suggest two spanning versions of the Chvátal-Erdős Theorem and a spanning version of Menger's Theorem (3.2.7). It is clear that higher powers of a graph will usually have better spanning connectedness. We try to give a quantitative description on how the operation of taking powers will improve the spanning connectedness from the viewpoint of the Hamiltonian thickness (\$3.2.8). In contrast to Eq. (3), the results in $\$ 3.2 .7$ and $\$ 3.2 .8$ provide lower bounds for Hamiltonian thickness in terms of graph connectivity. Hopefully, the work in $\$ 3.1$ and $\$ 3.2$ as well as many open questions gleaned in $\$ 3.3$. which are motivated by these work, can justify the effort to find, describe, and understand the vertex orderings with high Hamiltonian thickness and may cast new light on some apparently complex situations regarding various spanning connectedness properties.

The rest of this paper is organized as follows. In $\$ 2$, we discuss various kinds of spanning connectedness properties of graphs as well as the necessary terminology around them. Next we display all our main results and conjectures in $\$ 3$, where we only supply some short easy proofs of a few facts. Especially, the Normal Path Algorithm, a major tool for us in exposing the structures of interval graphs in this paper and a closely related one (74), is introduced in 3.2 .1 . In $\$ 4$ we provide all missing proofs for our results reported in $\sqrt{3}$

\section{Spanning connectedness properties}

In this paper, all graphs are assumed to be finite, simple, undirected and loopless. Let $G$ be a graph with vertex set $V(G)$ and edge set $E(G)$. Let $\mathcal{E}$ be a subset of $\left(\begin{array}{c}V(G) \\ 2\end{array}\right)$. We denote the graph $(V(G), E(G) \backslash \mathcal{E})$ by $G-\mathcal{E}$ and denote the graph $(V(G), E(G) \cup \mathcal{E})$ by $G+\mathcal{E}$. If $\mathcal{E}$ consists of one element $x y$, we will often write $G-x y$ and $G+x y$ in place of $G-\mathcal{E}$ and $G+\mathcal{E}$, respectively. For $\mathcal{E} \subseteq E(G), G[\mathcal{E}]$ stands for the graph $G-(E(G) \backslash \mathcal{E})$. 


\subsection{Endpoint constraints}

A graph that contains a Hamiltonian path is called a traceable graph. A graph is Hamiltonian provided it has a Hamiltonian cycle. A Hamiltonian-connected graph is a graph with at least two vertices in which every two distinct vertices are the endpoints of a Hamiltonian path. A graph is Hamiltonianconnected from a vertex $v \in V(G)$ if there is a Hamiltonian path from $v$ to every other vertex of $G$; See Dean et al. (31). A semi-Hamiltonian-connected graph is a graph in which every vertex is an endpoint of a Hamiltonian path. Let $k$ be a positive integer. A graph $G$ is spanning $k$-fan-connected if it has at least $k+1$ vertices and contains a spanning $k$ - $(x, U)$-fan for every choice of $x \in V(G)$ and $U \in\left(\begin{array}{c}V(G) \backslash\{x\} \\ k\end{array}\right)$; See Lin et al. (78). The local rail connectivity between distinct vertices $x$ and $y$ in a graph $G$ is the maximum number of pairwise internally disjoint $x, y$-paths, denoted $p_{G}(x, y)$; See Bondy and Murty (9, p. 206). For $\{x, y\} \in\left(\begin{array}{c}V(G) \\ 2\end{array}\right)$, define the local spanning rail connectivity between $x$ and $y$ to be $\widehat{p}_{G}(x, y)=\max \{k$ : there exists a spanning $t$-rail between $x$ and $y$ in $G$ for every $t \in[k]\}$ if there exists a spanning rail between $x$ and $y$ in $G$ and let $\widehat{p}_{G}(x, y)=0$ otherwise. A graph is spanning $k$-rail-connected if it has at least two vertices and there exists a spanning $k$-rail between every two different vertices of the graph; See Albert et al. (2), Lin et al. (76, 77, 78). Note

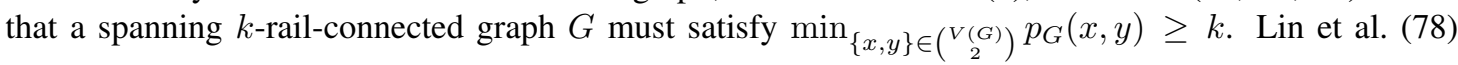
considered the so-called spanning pipeline-connectivity of a graph. As a rooted version of this concept, we call a graph $G$ with $|V(G)| \geq k+1$ rooted $k$-connected provided for any given set of $k$ vertex pairs $R=\left\{\left(s_{i}, t_{i}\right) \in V(G) \times V(G): i \in[k]\right\}$ there exists a spanning $k$-path system rooted at $R$. A graph $G$ with $|V(G)| \geq k+1$ is weakly rooted $k$-connected provided for any given set of $k$ vertex pairs $R=\left\{\left(s_{i}, t_{i}\right) \in\left(\begin{array}{c}V(G) \\ 2\end{array}\right): i \in[k]\right\}$ there exists a spanning $k$-path system rooted at $R$.

Let $H$ be a multigraph, possibly with loops, and let $G$ be a graph. For any map $f$ from $V(H)$ to $V(G)$, a spanning $H$-subdivision in $G$ with root $f$ is a set $\mathscr{S}$ of internally disjoint paths $g_{a b}$ in $G$ where $a b$ runs through all multiple edges of $H$ and $g_{a b}$ is an $f(a), f(b)$-path such that every vertex of $G$ appears in at least one path from $\mathscr{S}$ and it holds for all $v \in V(H)$ that $f(v)$ never appears as an interior vertex of any path from $\mathscr{S}$. Gould and Whalen (41) observed that many spanning connectedness problems can be unified as the problem of showing the existence of a spanning subdivision of a given multigraph $H$. For example, when $H$ is a multigraph with two vertices and several edges between the two vertices then a spanning $H$-subdivision is basically a spanning rail with given endpoints, and when $H$ is a star graph the spanning $H$-subdivision is basically a spanning fan with given endpoints. If the graph $H$ has in total $k$ multiple edges, it is not hard to see that the existence of a spanning $H$-subdivision in a graph $G$ amounts to the existence of a corresponding spanning $k$-path system. Moreover, depending on whether or not $H$ has loops and whether or not the root map $f$ is required to be injective, the existence of spanning $H$-subdivisions in $G$ with all "possible" root maps is equivalent to the requirement that $G$ is rooted $k$-connected or weakly rooted $k$-connected with corresponding "freely" chosen root set.

The spanning rail connectivity of a graph $G$, which we denote by $\operatorname{src}(G)$, is the largest integer $q$ such that $G$ is spanning $k$-rail-connected for all integers $k \in[q]$. Note that $\operatorname{src}(G)=0$ means that $G$ is not Hamiltonian-connected. The spanning fan connectivity of a graph $G$, denoted $\operatorname{sfc}(G)$, is the largest integer $q$ such that $G$ is spanning $k$-fan-connected for all integers $k \in[q]$. For $\operatorname{src}(G)$, we refer the reader to Chen et al. (21), Hsu and Lin (50), Huang and Hsu (52), Lin et al. (78) while for $\operatorname{sfc}(G)$ we refer the reader to Lin et al. (78). For a Hamiltonian-connected graph $G$, it is not hard to see the following claim of Lin et al. (78, Theorem 1):

$$
\kappa(G) \geq \operatorname{src}(G) \geq \operatorname{sfc}(G) .
$$


Given a graph $G$ and a vertex set $S \in\left(\begin{array}{c}V(G) \\ k\end{array}\right)$, a $k$-fixed-endpoint path cover of the graph $G$ with respect to $S$ is a path cover of $G$ such that the vertices from $S$ all appear as endpoints of the paths in the path cover. The $k$-fixed-endpoint path cover problem, namely the $k \mathrm{PC}$ problem, for a graph $G$ is to determine a $k$-fixed-endpoint path cover of $G$ of minimum size for any input $S \in\left(\begin{array}{c}V(G) \\ k\end{array}\right)$. The $k$-fixed-endpoint Hamiltonian path problem, namely the $k \mathrm{HP}$ problem, for a graph $G$ is to determine whether or not a $k$-fixed-endpoint path cover of $G$ of size one exists for any specific input $S \in\left(\begin{array}{c}V(G) \\ k\end{array}\right)$ and output such a Hamiltonian path if it does exist. Note that $k \mathrm{HP}$ can have a positive solution only if $k \leq 2$. Damaschke (29) indicated in 1993 that no polynomial algorithm is known for the 1HP problem or 2HP problem on interval graphs. Asdre and Nikolopoulos (4) discovered an $O\left(|V(G)|^{3}\right)$ time algorithm for the 1PC problem on any interval graph $G$, thus also solving the $1 \mathrm{HP}$ problem posed by Damaschke about 20 years ago. $\mathrm{Li}$ and $\mathrm{Wu}(74)$ recently improve the work of Asdre and Nikolopoulos (4) by designing an $O(|V(G)|+|E(G)|)$ time algorithm for the 1PC problem on any interval graph $G$. We remark that many concepts and results in this paper are motivated by the analysis and the design of this linear-time algorithm as reported in $\mathrm{Li}$ and $\mathrm{Wu}(74)$.

\subsection{Packability}

A linear forest is a forest of which every component is a path. The size of a linear forest is the sum of its number of edges and its number of length-zero paths (isolated vertices). For any graph $G, \partial(G)$ and $\Im(G)$ stand for the set of degree 1 vertices in $G$ and the set of vertices in $G$ whose degrees are at least two, respectively. Given $\mathcal{E} \subseteq E(G)$, we often write $\partial(\mathcal{E})$ for $\partial(G[\mathcal{E}])$ and write $\Im(\mathcal{E})$ for $\Im(G[\mathcal{E}])$ if no confusion will arise.

For a graph $G$ on $n$ vertices, the spanning connectedness problem is to find a sandwich graph $H$ between $\overline{K_{n}}$ and $G$, namely $\overline{K_{n}} \subseteq H \subseteq G$, which is of certain basic connection pattern, say a cycle or a path. It is natural to consider the more constrained problem obtained by replacing $\overline{K_{n}}$ by a subgraph of $G$ which is of some specific form, say being a linear forest. That is to say, we are interested in those graphs which possess a spanning graph of some connection pattern into which a given subgraph of some specific form can be packed.

A graph $G$ is $k$-packable Hamiltonian if for each linear forest $F$ in $G$ of size $k$ or less, there is a Hamiltonian cycle containing all the edges of $F$; See Pósa (92). A graph $G$ is $k$-packable traceable if for every subgraph $F$ of $G$ which is a linear forest of size at most $k$, there is a Hamiltonian path in $G$ containing all the edges of $F$. A graph $G$ is $k$-packable Hamiltonian-connected if for every subgraph $F$ of $G$ which is a linear forest of size at most $k$ and for any two vertices $u, v \in V(G) \backslash \Im(F)$ which do not fall into the same component of $F$, there is a spanning $u, v$-path in $G$ containing all the edges of $F$.

\subsection{Ordering constraints}

Let $k$ be a positive integer and let $G$ be a graph with at least $k$ vertices.

- The graph $G$ is $k$-vertex-ordered Hamiltonian if $G$ has at least $k$ vertices and for every sequence $v_{1}, \ldots, v_{k}$ of distinct vertices of $G$ there is a Hamiltonian cycle which encounters $v_{1}, \ldots, v_{k}$ cyclically in this order. Relevant discussions can be found in Chen et al. (18), Kierstead et al. (63), Ng and Schultz (

- The graph $G$ is $k$-vertex-ordered Hamiltonian-connected provided $G$ has at least $k+2$ vertices and for every sequence of $k$ distinct vertices $v_{1}, \ldots, v_{k}$, and every two distinct vertices $u$ and $u^{\prime}$ 
such that $u \notin\left\{v_{2}, \ldots, v_{k}\right\}$ and $u^{\prime} \notin\left\{v_{1}, \ldots, v_{k-1}\right\}, G$ contains a Hamiltonian path that starts at $u$, ends at $u^{\prime}$, and encounters the vertices $v_{1}, \ldots, v_{k}$ in this order. See $\mathrm{Ng}$ and Schultz (85) for some results about this property.

For any $k \geq 3$, according to $\mathrm{Ng}$ and Schultz (85, Proposition 1), a $k$-vertex-ordered Hamiltonian graph must be $(k-1)$-connected. $\mathrm{Ng}$ and Schultz $(85$. Theorem 10) also claimed that every Hamiltonian graph is 3 -vertex-ordered Hamiltonian and for any $k \geq 3$, every $(k-1)$-vertex-ordered Hamiltonianconnected graph is a $k$-vertex-ordered Hamiltonian graph. Moreover, for each integer $k \geq 4$, there exists a $(k-2)$-vertex-ordered Hamiltonian-connected graph that is not $k$-vertex-ordered Hamiltonian; $\mathrm{See} \mathrm{Ng}$ and Schultz (85, Theorem 12).

Besides passing through a sequence of vertices in order, we are also interested in the existence of a Hamiltonian cycle/path which passes through the components of a linear forest in arbitrarily prescribed order. Let us introduce the relevant spanning connectedness properties as below. To this end, we first prepare some terminology. Let $G$ be a graph and $F$ be a subgraph of $G$ which is a linear forest. Assume that $\mathscr{O}$ gives an ordering of the components of $F$, say $\mathscr{O}_{1}, \ldots, \mathscr{O}_{\ell}$. We assert that a path (cycle) $P$ of $G$ passes through $F$ according to $\mathscr{O}$ provided $F \subseteq P$ and along $P$ you will meet the components $\mathscr{O}_{1}, \ldots, \mathscr{O}_{\ell}$ in this order from one endpoint to the other endpoint (cyclically). We may think of the graph $F$ along with the ordering $\mathscr{O}$ of its components as an ordered structure and directly say a path/cycle passes through the ordered linear forest $F$ with the ordering $\mathscr{O}$ in mind. Note that a path passes through the ordered linear forest $\mathscr{O}_{1}, \ldots, \mathscr{O}_{\ell}$ if and only if it passes through its reversal $\mathscr{O}_{\ell}, \ldots, \mathscr{O}_{1}$.

Fix a positive integer $k$ and let $G$ be a graph with at least $k$ vertices.

- The graph $G$ is $k$-ordered Hamiltonian if it has at least $k$ vertices and for every linear forest $F$ in $G$ of size at most $k$ and any ordering of the components of $F$, there is a Hamiltonian cycle of $G$ which is a supergraph of $F$ and encounters the components of $F$ in that specified order, namely the Hamiltonian cycle traverses $F$ in the given ordering.

- The graph $G$ is $k$-ordered traceable if it has at least $k$ vertices and for every linear forest $F$ in $G$ of size at most $k$ and any ordering of the components of $F$, there is a Hamiltonian path of $G$ which passes through $F$ in that specified ordering.

- The graph $G$ is $k$-ordered Hamiltonian-connected provided it has at least $k+2$ vertices and for every linear forest $F$ of size at most $k$ contained in $G$ as well as an ordering $\mathscr{O}_{1}, \ldots, \mathscr{O}_{\ell}$ of its components, for every two vertices $x$ and $y$ such that $x \in(V(G) \backslash V(F)) \cup\left(V\left(\mathscr{O}_{1}\right) \backslash \Im\left(\mathscr{O}_{1}\right)\right)$, $y \in(V(G) \backslash V(F)) \cup\left(V\left(\mathscr{O}_{\ell}\right) \backslash \Im\left(\mathscr{O}_{\ell}\right)\right)$ and no component of $F$ contains both $x$ and $y$, we can find a Hamiltonian path of $G$ which leads from $x$ to $y$ and traverses through the ordered linear forest $F$.

To go one step further, for each path of positive length in the linear forest $F$, we may not only request the existence of an interconnection structure which passes through the components of $F$ in a given ordering but may even impose the direction of passing through each component of $F$.

\subsection{Extension property}

A graph $G$ is $k$-HC-extendable if it contains a path of length $k$ and if every such path is contained in some Hamiltonian cycle of $G$; A graph $G$ is strongly- $k$-HP-extendable if it contains a path of length $k$ and if every such path $P$ can be extended into a Hamiltonian path of $G$ that starts with $P$. Both of these 
properties are considered by Miklavič and Šparl (83). A graph on $n$ vertices is cycle extendable (path extendable) if for every cycle (path) $C$ of less than $n$ vertices there is another cycle (path) containing all vertices of $C$ plus a single new vertex. A graph on $n$ vertices is $k$-rail extendable if for every $k$-rail $C$ of less than $n$ vertices there is another $k$-rail which has the same root as $C$ and contains all vertices of $C$ plus a single new vertex.

To display the existence of some spanning pattern, a common two-step approach, as suggested by Gould and Whalen (41), consists of firstly showing that given conditions are sufficient to imply the existence of a particular subgraph and then demonstrating that the existing subgraph can be extended till it becomes a spanning subgraph. In this sense, we can view those extension properties as spanning connectedness properties.

\subsection{Fault-tolerance}

Let $G$ be a graph and let $k$ be a nonnegative integer. A graph $G$ is $k$-edge-fault-tolerant Hamiltonian if $G-\mathcal{E}$ is Hamiltonian for every set $\mathcal{E} \in\left(\begin{array}{c}E(G) \\ <k\end{array}\right)$; See Harary and Hayes (43), Hsieh et al. (48), Huang et al. (53). A graph $G$ is $k$-vertex-fault-tolerant Hamiltonian if $G-\mathcal{V}$ is Hamiltonian for every set $\mathcal{V} \in\left(\begin{array}{c}V(G) \\ \leq k\end{array}\right)$; Related research includes Chartrand et al. (14), Lai and Shao (68). A graph $G$ is $k$-faulttolerant Hamiltonian if $G-F$ is Hamiltonian for every set $F \in\left(\begin{array}{l}V(G) \cup E(G) \\ <k\end{array}\right)$; See Su et al. (103). A graph $G$ is $k$-vertex-fault-tolerant Hamiltonian-connected if the deletion of every set of at most $k$ vertices from $G$ results in a Hamiltonian-connected graph; See Chen and Quimpo (16), Hu et al. (51), Lai et al. (69), Ore (87). A graph $G$ is exact- $k$-edge-fault-tolerant Hamiltonian if $G-\mathcal{E}$ is Hamiltonian for every set $\mathcal{E} \in\left(\begin{array}{c}E(G) \\ k\end{array}\right)$. A graph $G$ is exact- $k$-vertex-fault-tolerant traceable if $G-\mathcal{V}$ is traceable for every set $\mathcal{V} \subseteq\left(\begin{array}{c}V(G) \\ k\end{array}\right)$ - such a graph is called $(|V(G)|-k)$-traceable in Bullock et al. (12). Similarly, we can define $k$-edge-fault-tolerant traceable graphs, $k$-fault-tolerant spanning $t$-rail-connected graphs, $k$-fault-tolerant spanning $t$-fan-connected graphs, etc.

For any two vertices $x$ and $y$ of a graph $G$, an $x, y$-vertex-cut in $G$ is a subset $S$ of $V(G) \backslash\{x, y\}$ such that $x$ and $y$ belong to different components of $G-S$. The minimum size of a vertex cut separating $x$ and $y$ is denoted by $c_{G}(x, y)$. We make the convention that $c_{G}(x, y)=\infty$ whenever $x y \in E(G)$. This function $c$ is called the local cut function on $G$. For any pair of distinct vertices $x$ and $y$ in $G$ and any positive integer $k$, the $k$ th local spanning cut function $\widehat{c}_{G}^{k}(x, y)$ is the minimum size of a set $S \subseteq V(G) \backslash\{x, y\}$ such that $G-S$ does not have a spanning $k$-rail connecting $x$ and $y$. We understand that $\widehat{c}_{G}^{1}(x, y)=\infty$ when $G$ is a complete graph and we can see that $\widehat{c}_{G}^{1}(x, y)<\infty$ when $G$ is not a complete graph. In addition, let us define the local spanning cut function for any pair of distinct vertices $x$ and $y$ in $G$ to be $\widehat{c}_{G}(x, y)=\max _{k} \widehat{c}_{G}^{k}(x, y)$, which is the minimum size of a set $S \subseteq V(G) \backslash\{x, y\}$ such that $G-S$ does not have any spanning rail between $x$ and $y$.

Note that if $G$ is $k$-vertex-fault-tolerant traceable, then it holds $\pi(G-S) \leq \max \{1,1+|S|-k\}$ for every $S \subseteq V(G)$. It is also easy to see that a graph must be $(k+2)$-connected if it is $k$-vertex-faulttolerant Hamiltonian. The converse is not true, as there exist arbitrarily highly connected nonhamiltonian graphs, say $K_{n, n+1}$ (also see Example 59).

\subsection{Passing through specified edges with fault-tolerance}

Let $G$ be a graph. The graph $G$ is $k$-vertex-super-Hamiltonian provided, for all nonnegative integers $t_{1}$ and $t_{2}$ such that $t_{1}+t_{2} \leq k$, the removal of every $t_{1}$ vertices from $G$ results in a $t_{2}$-packable Hamiltonian graph. The graph $G$ is $k$-vertex-super-traceable provided, for all nonnegative integers $t_{1}$ and $t_{2}$ such that 
$t_{1}+t_{2} \leq k$, the removal of arbitrarily given $t_{1}$ vertices from $G$ results in a $t_{2}$-packable traceable graph. The graph $G$ is $k$-vertex-super-Hamiltonian-connected provided, for all nonnegative integers $t_{1}$ and $t_{2}$ such that $t_{1}+t_{2} \leq k$, for each subset $\mathcal{V} \subseteq V(G)$ of size $t_{1}$ and each set $\mathcal{E} \subseteq E(G-\mathcal{V})$ such that $G[\mathcal{E}]$ is a linear forest of totally $t_{2}$ edges and for every two vertices $u$ and $v$ which lie in $V(G-\mathcal{V}) \backslash \Im(\mathcal{E})$ and do not appear in the same component of $G[\mathcal{E}], G-\mathcal{V}$ contains a spanning $u, v$-path passing through all edges in $\mathcal{E}$. The graph $G$ is $k$-super-Hamiltonian provided for all nonnegative integers $t_{1}, t_{2}$ and $t_{3}$ such that $t_{1}+t_{2}+t_{3} \leq k$, for every set $\mathcal{V} \subseteq V(G)$ of size $t_{1}$, every set $\mathcal{F} \subseteq E(G-\mathcal{V})$ of size $t_{2}$ and every set $\mathcal{E} \subseteq E(G-\mathcal{V}) \backslash \mathcal{F}$ such that $G[\mathcal{E}]$ is a linear forest of totally $t_{3}$ edges, $G-\mathcal{V}-\mathcal{F}$ contains a Hamiltonian cycle passing through all edges in $\mathcal{E}$. In similar fashion, we can define a graph to be $k$-super-Hamiltonian-connected, $k$-super-traceable, and so on.

\section{Main results}

\subsection{General graphs}

\subsubsection{Spanning connectedness properties}

Theorem 1 Let $k$ be a positive integer and let $G$ be an $n$-vertex graph possessing a $k$-thick Hamiltonian vertex ordering $\pi$. Then $G$ has a spanning $k$-rail between $\pi_{1}$ and $\pi_{n}$. What is more, we can require that $\pi_{n-1}$ is adjacent to $\pi_{n}$ on one of the $k$ paths in this spanning $k$-rail.

We mention that a generalization of Theorem 1 has been proved in $\mathrm{Wu}$ et al. (107): For any integers $k \geq t \geq 2$, if a graph $G$ has a $k$-thick Hamiltonian vertex ordering $\pi$, then $\widehat{c}_{G}^{t}\left(\pi_{1}, \pi_{|V(G)|}\right) \geq k-t+1$.

The next result is immediate from Theorem 1 . An alternative proof without using Theorem 1 will be presented in $\$ 4.1 .1$ and that proof is closer to the idea used in the linear-time algorithm of $\mathrm{Li}$ and $\mathrm{Wu}(74)$ for solving the 1PC problem on interval graphs.

Corollary 2 Suppose that $G$ is a graph from $\digamma^{2}$. Then, for any $v \in V(G)$, the graph $G$ has a Hamiltonian path with $v$ as an endpoint, namely $1 H P$ is always solvable on $G$.

Example 3 Let $G$ be the graph on eight vertices as depicted in Fig. 1] It is a routine matter to derive that $G[i, \ldots, 7]$ is Hamiltonian for each $i \in[5]$. Furthermore, one can check that $G$ has no 2-thick Hamiltonian vertex ordering. This means that the converse of Theorem 1 is not true when $k=2$. Note that $G$ is neither a chordal graph nor an AT-free graph (and so not a cocomparability graph by Lemma 106.).

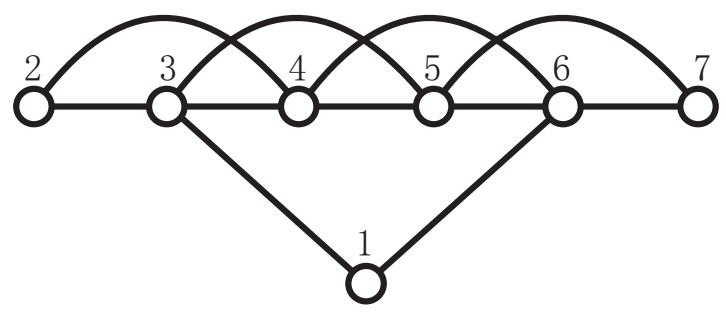

Fig. 1: The graph $G$ in Example 3 
For any graph $G$ and any $W \subseteq V(G)$, write $N_{G}(W)$ for $\left(\cup_{v \in W} N_{G}(v)\right) \backslash W$ and write $N_{G}[W]$ for $N_{G}(W) \cup W$. If a graph $G$ with $n$ vertices has a $k$-thick vertex ordering $\pi$, surely the size of $N_{G}\left(\left\{\pi_{1}, \ldots, \pi_{i}\right\}\right)$ is greater than or equal to $k$ for each $i \in[n-k]$, but not vice versa. The next example says that we could not replace the $k$-thickness condition by the latter weaker condition in Theorem 1.

Example 4 Take an integer $n \geq 5$ and let $G$ be the graph with $V(G)=\left\{\pi_{i}: i \in[n]\right\}$ and $E(G)=$ $\left\{\pi_{1} \pi_{n-1}, \pi_{2} \pi_{n}\right\} \cup\left\{\pi_{i} \pi_{i+1}: i \in[n-1]\right\}$. For every $i \in[n-2]$, it holds $\left|N_{G}\left(\left\{\pi_{1}, \ldots, \pi_{i}\right\}\right)\right| \geq 2$ and $\left|N_{G}\left(\left\{\pi_{n}, \ldots, \pi_{n+1-i}\right\}\right)\right| \geq 2$. But $G$ has no Hamiltonian cycle, namely no spanning 2-rail between $\pi_{1}$ and $\pi_{n}$.

Based on the concept of degree sequence, many sufficient conditions for the existence of a Hamiltonian cycle or a Hamiltonian path with two arbitrarily fixed endpoints have been developed. See Ozeki and Yamashita (88) and its references. Theorems 5,7 and 9 shed a little light on establishing corresponding results in terms of forward degree sequence.

Theorem 5 Let $k$ be an integer not less than 2. If $G \in \digamma^{k}$ holds, then $G$ is $(k-2)$-vertex-superHamiltonian.

Theorem 5 has the following direct consequence.

Corollary 6 Let $k \geq 2$ be an integer and let $G \in \digamma^{k}$. Take $\mathcal{V} \subseteq V(G)$ and $\mathcal{E} \subseteq E(G-\mathcal{V})$ such that $G[\mathcal{E}]$ is a linear forest and $|\mathcal{E}|+|\mathcal{V}| \leq k-2$. Then, for any vertex $v \in V(G-\mathcal{V}) \backslash \Im(\mathcal{E}), G-\mathcal{V}$ contains a Hamiltonian path starting from $v$ and passing through all the edges in $\mathcal{E}$. (See 2.2 for the definition of $\Im(\mathcal{E})$ ).

Theorem 7 For any positive integer $k$, all graphs $G \in \digamma^{k}$ are $(k-1)$-vertex-super-traceable.

Example 8 Take two integers $t$ and $n$ such that $t>1$ and $n \geq 3$. Consider the complete multipartite graph $K_{t ; n}$ whose vertex set is the union of $n$ disjoint $t$-sets $V_{1}, \ldots, V_{n}$ and there is an edge between $u$ and $v$ if and only if there exit $i \neq j$ such that $u \in V_{i}$ and $v \in V_{j}$. We can check that deleting any vertex subset of size at most $t+1$ from $K_{t ; n}$ yields a traceable graph while the Hamiltonian thickness of $K_{t ; n}$ is $n-1$.

Theorem 9 If $k$ is an integer greater than 2 , then every graph $G \in \digamma^{k}$ is $(k-3)$-vertex-super-Hamiltonianconnected.

$\mathrm{Ng}$ and Schultz (85, Problem 4) suggested to study the existence of small degree $k$-vertex-ordered Hamiltonian-connected graphs. The next result provides a method to construct such graphs.

Theorem 10 Let $k$ be a positive integer. If $G \in \digamma^{2 k+2}$, then $G$ is $k$-ordered Hamiltonian-connected.

Corollary 11 Let $k$ be a positive integer. If $G \in \digamma^{2 k}$, then $G$ is $k$-ordered traceable.

Theorem 12 Let $k$ be a positive integer. If $G \in \digamma^{2 k+1}$, then $G$ is $k$-ordered Hamiltonian.

The following result shows a link between Hamiltonian thickness and the general spanning multigraph subdivision problem as discussed in 2.1 .

Theorem 13 Let $k$ be a positive integer and pick $G \in \digamma^{2 k+1}$. Then $G$ is weakly rooted k-connected. 
Let $k$ be an integer not less than 3 and pick $G \in \digamma^{k}$. An immediate consequence of Theorem 13 is that

$$
\min \{\operatorname{sfc}(G), \operatorname{src}(G)\} \geq\left\lfloor\frac{k-1}{2}\right\rfloor .
$$

Incorporating some new idea, Wu et al. (107) deduced the stronger results that $\operatorname{sfc}(G) \geq k-1$ and $\operatorname{src}(G) \geq k$. When $G$ is specified to be an interval graph, Theorem 37 iii) to be presented in $\$ 3.2 .4$ will give even more exact information on $\operatorname{sfc}(G)$ and $\operatorname{src}(G)$.

Corollary 14 Let $G$ be a graph and let $k$ be a positive integer. If $G \in \digamma^{2 k-1}$, then $G$ is $k$-vertex-ordered traceable.

\subsubsection{Scattering number and Hamiltonian thickness}

A graph $G$ is mottling if either $G=K_{1}$ or

$$
\pi(G)=\max \{1, \operatorname{sc}(G)\}
$$

By Eq. (2) and the definition of Hamiltonian thickness, we know that $G$ is mottling if and only if $G$ is either traceable or fulfils $2=\operatorname{sc}(G)+\mathcal{H}(G)$.

We are ready to define several graph classes. Note that in the definitions of the first three graph classes given below we could replace "if and only if" by merely "if", since the "only if" part holds always for every graph. Let $\mathcal{C}$ be a graph class.

- The graph class $\mathcal{C}$ is path-tough if, for every $G \in \mathcal{C}, G$ is traceable if and only if $\operatorname{sc}(G) \leq 1$. See Kratsch et al. (67, Definition 2.1).

- We call $\mathcal{C}$ cycle-tough if, for every $G \in \mathcal{C}$ with $|V(G)| \geq 3, G$ is Hamiltonian if and only if $\operatorname{sc}(G) \leq 0$ (or equivalently, $\mathrm{t}(G) \geq 1$ ). See Kratsch et al. (67, Definition 2.1).

- The graph class $\mathcal{C}$ has the Deogun-Kratsch-Steiner property if, for every $G \in \mathcal{C}$ with $|V(G)| \geq$ $3, G$ is Hamiltonian if and only if $G-v$ is traceable for every $v \in V(G)$. See Deogun et al. (32), Kratsch et al. (67).

- The graph class $\mathcal{C}$ is a Jung's family if every graph $G \in \mathcal{C}$ is mottling, is Hamiltonian when $\operatorname{sc}(G)=0$, and is Hamiltonian-connected when $\operatorname{sc}(G) \leq-1$; See Giakoumakis et al. (38), Jung (60). Giakoumakis et al. (38, Definition 1) called every graph from a Jung's family a Jung graph.

The graph classes specified by the above-mentioned spanning connectedness properties may support quite well the design of polynomial-time certifying recognition algorithms as they possess good positive certificate and negative certificate, as argued by Deogun et al. (32, p. 100).

Cocomparability graphs are path-tough by Lehel (72), are cycle-tough by Deogun et al. (32, Theorem 10), and have the Deogun-Kratsch-Steiner property by Deogun et al. (32, Theorem 9). Hochstättler and Tinhofer (47, Theorem 2) showed that $P_{4}$-extendible graphs are mottling. In addition, Jung graphs include $P_{4}$-free graphs (cographs) according to Jung (60) and cocomparability graphs according to Deogun et al. (32. Theorem 8). For every $P_{4}$-tidy graph, a good discussion on when it is a Jung graph is given in Giakoumakis et al. (39). In the process of giving a linear-time certifying algorithm for the minimum path cover problem on interval graphs, Hung and Chang (54, Corollary 3.3) again confirmed that interval 
graphs, a special class of cocomparability graphs, are mottling. Note that our Theorem 33 will be a strengthening of this fact.

In $\$ 3.1 .1$, we suggested that a thickest Hamiltonian vertex ordering of a graph may serve as a positive certificate of its various spanning connectedness. We now propose that, as already hinted by the previous discussion in this subsection, a scattering set may often play the role of a negative certificate for several spanning connectedness properties for some graph classes, especially those graphs $G$ for which we know $\operatorname{sc}(G)+\mathcal{H}(G)$ in advance from their structural properties.

In light of Eq. (2), the first inequality in the next proposition is a small improvement of Eq. (1). The second inequality in Theorem 15 should be in the folklore.

Theorem 15 For every graph $G$, it holds $\operatorname{sc}(G)+\mathcal{H}(G) \leq 2 \leq \operatorname{sc}(G)+\kappa(G)$.

Deogun et al. (32) defined the scattering number of a graph in a way that is a bit different with ours. However, it can be checked that the proof of Deogun et al. (32, Proposition 7) can be adapted a little to give

$$
\operatorname{sc}(G)=\left\{\begin{aligned}
\max _{\substack{v \in V(G) \\
G-v \text { is not a complete graph }}} \operatorname{sc}(G-v)-1, & \text { if } G \text { is not a complete graph, } \\
\max _{v \in V(G)} \operatorname{sc}(G-v)-1, & \text { if } G \text { is a complete graph, }
\end{aligned}\right.
$$

where $G$ is any connected graph with at least two vertices. This fact will be used in our proof of Theorem 16. Note that, thanks to Theorem 15, a graph $G$ for which equality in Eq. (3) holds, i.e., $\mathcal{H}(G)=\kappa(G)$, must satisfy $\operatorname{sc}(G)+\mathcal{H}(G)=2$.

Theorem 16 Let $\mathcal{C}$ be a graph class such that $\operatorname{sc}(G)+\mathcal{H}(G)=2$ holds for all $G \in \mathcal{C}$.

i) The graph class $\mathcal{C}$ is path-tough;

ii) The graph class $\mathcal{C}$ is cycle-tough;

iii) The graph class $\mathcal{C}$ is a Jung's family;

iv) The graph class $\mathcal{C}$ has the Deogun-Kratsch-Steiner property.

Theorem 17 If $G$ is a forest, then $\operatorname{sc}(G)+\mathcal{H}(G)=2$.

Let $k$ be a positive integer and let $G$ be a graph with $n \geq k+1$ vertices. An ordering $\pi \in V(G)$ ! is said to be an exact- $k$-thick Hamiltonian vertex ordering of $G$ provided $\pi$ is a Hamiltonian vertex ordering and $\mathrm{d}_{G, \pi}(i)=\min \{k, n-i\}$ holds for all $i \in[n]$. We call $G$ an exact- $k$-thick Hamiltonian graph if it has an exact- $k$-thick Hamiltonian vertex ordering. An exact- $k$-thick Hamiltonian graph is also called a maximal $k$-degenerate graph in Lick and White (75).

Theorem 18 Let $k$ be a positive integer and let $G$ be a graph. Then $\operatorname{dgn}(G)=\mathcal{H}(G)=k$ if and only if $G$ is an exact-k-thick Hamiltonian graph. In addition, every exact- $k$-thick Hamiltonian graph $G$ satisfies $2-\operatorname{sc}(G)=\mathcal{H}(G)=\kappa(G)=\operatorname{dgn}(G)$.

Recall that any graph obtained from a graph $G$ by deleting one vertex from it is called a vertex-deleted subgraph of $G$. A graph is a hypotraceable if it is not traceable but every vertex-deleted subgraph of it is traceable; A graph is hypohamiltonian if it is nonhamiltonian but every vertex-deleted subgraph of it is Hamiltonian. We point out that there do exist hypotraceable/hypohamiltonian graphs of many vertices; 
See Thomassen (104), Wiener and Araya (105). A graph is hypo-Hamiltonian-connected if it is not Hamiltonian-connected but every vertex-deleted subgraph of it is Hamiltonian-connected.

Theorem 19 Let $G$ be a chordal graph with more than two vertices. Then $G$ is not hypotraceable, not hypohamiltonian and not hypo-Hamiltonian-connected.

Example 20 a) Let $G$ be a graph which is the disjoint union of two traceable graphs. Then $\operatorname{sc}(G)=2$ and $\mathcal{H}(G)=0$.

b) For any positive integer $m$, the graph $K_{2, m}$ is a cocomparability graph. When $m \geq 3$, we have $\operatorname{sc}\left(K_{2, m}\right)=m-2$ and $\mathcal{H}\left(K_{2, m}\right)=4-m$ and so $\operatorname{sc}\left(K_{2, m}\right)+\mathcal{H}\left(K_{2, m}\right)=2$. When $m=2$, $\operatorname{sc}\left(K_{2,2}\right)=0, \mathcal{H}\left(K_{2,2}\right)=1$ and so $\operatorname{sc}\left(K_{2,2}\right)+\mathcal{H}\left(K_{2,2}\right)=1$. Note that $K_{2, m}$ is traceable if and only if $m \leq 2$. Also recall that, as shown in Deogun et al. (32. Theorem 8), every cocomparability graph is mottling.

c) The Petersen graph $G$, also known as the Kneser graph $K G(5,2)$ or the Odd graph $O_{2}$, has vertex set $\left(\begin{array}{c}{[5]} \\ 2\end{array}\right)$ and $S, T \in\left(\begin{array}{c}{[5]} \\ 2\end{array}\right)$ are adjacent in $G$ if and only if $S \cap T=\emptyset$. It holds $\operatorname{sc}(G)=-1$ and $\mathcal{H}(G)=1$. For $\{S, T\} \in\left(\begin{array}{c}V(G) \\ 2\end{array}\right)$, there is a spanning $S, T$-path in $G$ if and only if $|S \cap T|=1$. The Petersen graph is the only hypohamiltonian graph with at most ten vertices; See Gaudin et al. (37), Thomassen (104).

d) Deleting any vertex subset of size at most 2 from the Petersen graph results in a traceable graph. But the Petersen graph does not have a 2-thick Hamiltonian path, not mentioning a Hamiltonian cycle. This means that the Petersen graph does not have the Deogun-Kratch-Steiner property.

e) (Ziqing Xiang) There are exactly three graphs $G$ with at most 9 vertices satisfying both

$$
\max _{v \in V(G)} \mathcal{H}_{G}(v)>\min _{v \in V(G)} \mathcal{H}_{G}(v)
$$

and

$$
\operatorname{sc}(G)+\mathcal{H}(G)<0 .
$$

We enumerate them as $G_{1}, G_{2}$ and $G_{3}$ in Fig. 2 One can check the following: $\left\{\mathcal{H}_{G_{1}}(v): v \in\right.$ $\left.V\left(G_{1}\right)\right\}=\{2,1\}, \operatorname{sc}\left(G_{1}\right)=-3 ;\left\{\mathcal{H}_{G_{2}}(v): v \in V\left(G_{2}\right)\right\}=\{3,2\}, \operatorname{sc}\left(G_{2}\right)=-4 ;\left\{\mathcal{H}_{G_{3}}(v): v \in\right.$ $\left.V\left(G_{3}\right)\right\}=\{4,3\}, \operatorname{sc}\left(G_{3}\right)=-5$.

f) Let $k$ be a positive integer and let $G_{k}$ be the disjoint union of $k$ copies of the graph $H$ with $V(H)=[6]$ and $E(H)=\{12,23,31,14,25,36\}$. It holds $\operatorname{sc}\left(G_{k}\right)=k, \mathcal{H}\left(G_{k}\right)=2-2 k$ and so $\operatorname{sc}\left(G_{k}\right)+$ $\mathcal{H}\left(G_{k}\right)=2-k$.

g) For the graph $G$ in Fig. 1 (Example 3), it holds $\operatorname{sc}(G)=0$ and $\mathcal{H}_{G}(v)=1$ for all $v \in V(G)$.

h) For the graph $G$ in Fig. 3 it holds $\operatorname{sc}(G)=-2$ and $\mathcal{H}_{G}(v)=1$ for all $v \in V(G)$.

Let us close this section with a result about the influence on the Hamiltonian thickness parameter by some graph modifications.

Theorem 21 Let $G$ be a graph with $\mathcal{H}(G) \leq 2 \leq|V(G)|$.

a) For every $v \in V(G)$, it holds $\mathcal{H}_{G}(v) \in\{\mathcal{H}(G), \mathcal{H}(G)-1\}$ and hence $\mathcal{H}(G-v) \geq \mathcal{H}(G)-1$.

b) For every e $\in E(G)$, it holds $\mathcal{H}(G-e) \in\{\mathcal{H}(G), \mathcal{H}(G)-1\}$. 


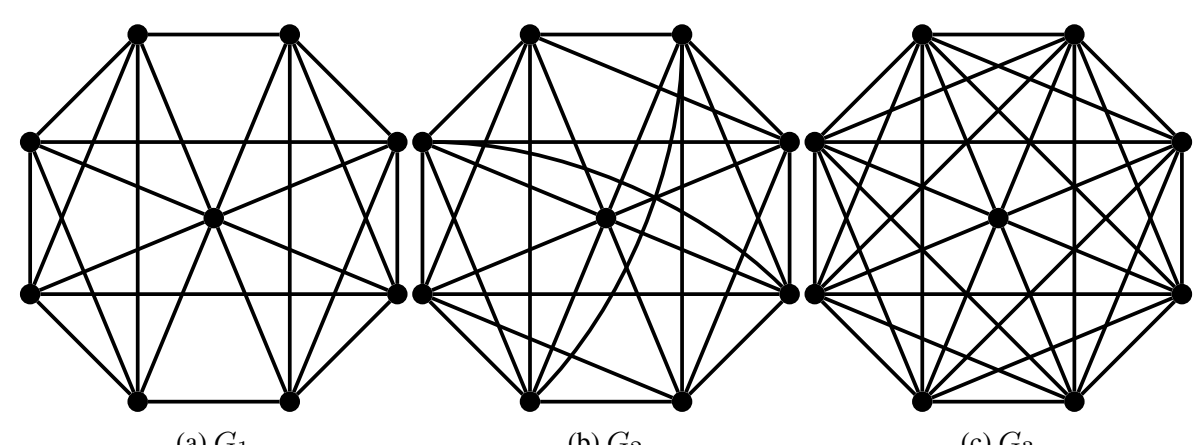

(a) $G_{1}$

(b) $G_{2}$

(c) $G_{3}$

Fig. 2: Three graphs $G$ satisfying both $\max _{v} \mathcal{H}_{G}(v)>\min _{v} \mathcal{H}_{G}(v)$ and $\operatorname{sc}(G)+\mathcal{H}(G)<0$.

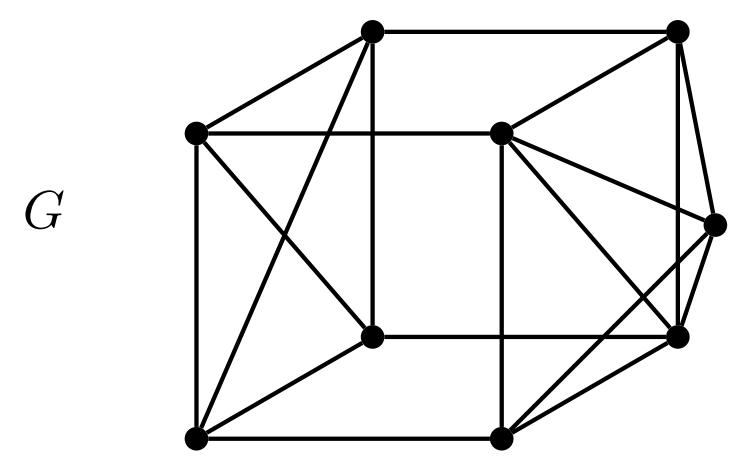

Fig. 3: $\max _{v \in V(G)} \mathcal{H}_{G}(v)=\min _{v \in V(G)} \mathcal{H}_{G}(v)=1, \operatorname{sc}(G)=-2$.

\subsection{Interval graphs}

According to Gould (42, $\S 3$ ), little has been done in developing existence of cycles in graph classes defined by forbidden subgraphs. We move on now to some discussions about interval graphs, which may form a very surprising exceptional graph class regarding the preceding statement. In 3.2 .1 we introduce a basic algorithm, which not only appears in the statement of some of our results but also plays an important role in our mathematical analysis of relevant problems. In the ensuing seven subsections we present our main work on interval graphs.

\subsubsection{Normal path algorithm}

We start with the following Normal Path Algorithm (Algorithm 3.1) for graph search, which is very similar to the rightmost neighbour algorithm proposed in Corneil et al. (27), Mertzios and Corneil (82). Roughly speaking, the algorithm greedily chooses the neighbor which appears earliest in a given vertex ordering at each step and grows a path that eventually snakes around the whole graph.

If the graph $G$ is from some structured graph class, the ordering $\xi$ is usually chosen to be a one 


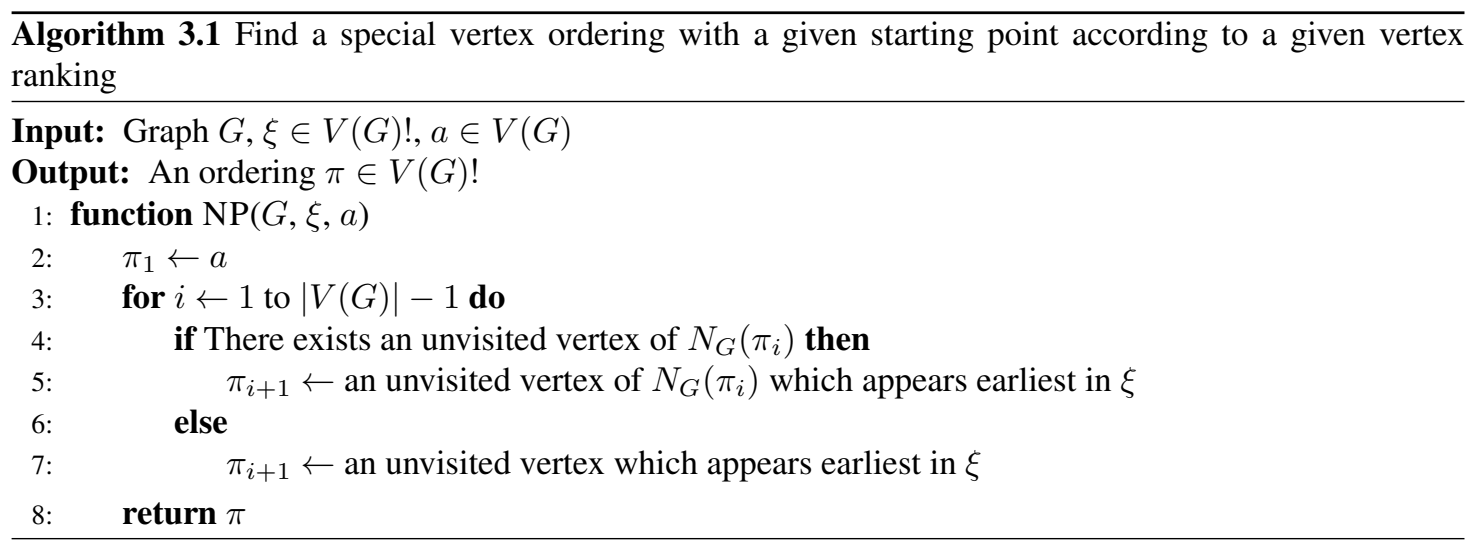

with some characteristic property, say a cocomparability ordering (umbrella-free ordering) suggested by Kratsch and Stewart (65, p. 402), a perfect elimination ordering defined by Dirac (33) or an interval ordering proposed by Raychaudhuri (93), respectively, if $G$ is a cocomparability graph, a chordal graph or an interval graph. We call an output of $\operatorname{NP}(G, \xi, a)$ a normal vertex ordering with respect to $\xi$ if $a=\xi(1)$. The normal path algorithm is very powerful in detecting the spanning connectedness of cocomparability graphs. For example, if a cocomparability graph has a Hamiltonian path, then it has a normal Hamiltonian vertex ordering with respect to an LDFS cocomp ordering as claimed by Mertzios and Corneil (82, Theorem 2), and every normal vertex ordering of $G$ with respect to an LDFS cocomp ordering is Hamiltonian by Corneil et al. (27, Theorem 4.14).

Let $\mathcal{I}$ be an interval representation of a graph $G$. With respect to the interval representation $\mathcal{I}$, we say that a maximal clique $Q$ of $G$ is to the left of another maximal clique $Q^{\prime}$ whenever $\cap_{v \in Q} \mathcal{I}(v)$ is an interval to the left of the interval $\cap_{v \in Q^{\prime}} \mathcal{I}(v)$, namely, $\min _{v \in Q} r_{\mathcal{I}}(v)<\max _{v \in Q^{\prime}} \ell_{\mathcal{I}}(v)$. Moreover, it is well-known that all maximal cliques of the interval graph $G$ can be listed as $Q_{1}, \ldots, Q_{r}$ such that $Q_{i}$ is to the left of $Q_{j}$ with respect to $\mathcal{I}$ if and only if $i<j$. We say $a \in V(G)$ is a leftmost vertex for $\mathcal{I}$ if $r_{\mathcal{I}}(a)=\min \left\{r_{\mathcal{I}}(v): v \in V(G)\right\}$ and we say $b \in V(G)$ is a rightmost vertex for $\mathcal{I}$ if $\ell_{\mathcal{I}}(b)=\max \left\{\ell_{\mathcal{I}}(v): v \in V(G)\right\}$. The right-endpoint ordering of $G$ according to $\mathcal{I}$ is an ordering $\pi$ of $V(G)$ such that $r_{\mathcal{I}}\left(\pi_{p}\right) \leq r_{\mathcal{I}}\left(\pi_{q}\right)$ whenever $p<q$. We will refer to this ordering $\pi$ as $\xi^{\mathcal{I}}$. If $a$ is a vertex belonging to the leftmost clique of $G$ with respect to $\mathcal{I}, \operatorname{NP}\left(G, \xi^{\mathcal{I}}, a\right)$ is the so-called Straight Path (Normal Path) Algorithm for interval graphs studied in Arikati and Pandu Rangan (3) and we will often write it as

$$
\operatorname{NP}(G, \mathcal{I}, a) \text {. }
$$

Note that the basic ideas of this algorithm also appear in Damaschke (29), Ioannidou et al. (56), Keil (62), Manacher et al. (80). An ordering of the vertex set of an interval graph generated by the normal path algorithm is called an almost normal vertex ordering. If we further require that $a$ is a leftmost vertex for $\mathcal{I}$ in the procedure $\operatorname{NP}(G, \mathcal{I}, a)$, then Damaschke (29) called the output ordering of $\operatorname{NP}(G, \mathcal{I}, a)$ a normal vertex ordering of $G$ with respect to $\mathcal{I}$.

\subsubsection{Characterizations of $\digamma^{k}$}

We need some more terminology before we can state our first main result on interval graphs. Two (notnecessarily distinct) vertices $u$ and $v$ of an interval graph $G$ form an opposite pair if one is a rightmost 
vertex and the other is a leftmost vertex of $G$ with respect to one of its interval representations. A typical $k$-rail of an interval graph is a spanning $k$-rail between opposite vertices. A typical $k$-fan of an interval graph with respect to an interval representation $\mathcal{I}$ of $G$ is a spanning $k$ - $(x ; U)$-fan where $x$ is a leftmost vertex and $U$ is a $k$-set lying inside the rightmost clique with respect to $\mathcal{I}$.

Theorem 22 For every interval graph $G$ and every positive integer $k<|V(G)|$, the following statements are equivalent.

a) For every interval representation $\mathcal{I}$ of $G$ and every opposite pair a and $b$ of $G$ with respect to $\mathcal{I}$, there exists a spanning $k$-rail of $G$ between $a$ and $b$.

b) The graph $G$ possesses a typical k-rail.

c) The graph $G$ is $(k-1)$-vertex-fault-tolerant traceable.

d) The graph $G$ is exact- $(k-1)$-vertex-fault-tolerant traceable.

e) All normal vertex orderings of $G$ are $k$-thick Hamiltonian vertex orderings.

f) The graph $G$ has a $k$-thick normal vertex ordering, which, surely, is a Hamiltonian vertex ordering.

g) The graph $G$ has a k-thick Hamiltonian vertex ordering, namely the graph $G$ lies in $\digamma^{k}$.

h) The graph $G$ possesses a typical k-fan.

Theorem $22 \mathrm{~b}$ ) $\Leftrightarrow \mathrm{c}$ ) for $k=2$ just says that interval graphs have the Deogun-Kratsch-Steiner property. Recall that, more generally, by Deogun et al. (32) Theorem 9), all cocomparability graphs have the Deogun-Kratsch-Steiner property.

Theorem 23 Let $G$ be a graph and let $\mathcal{I}$ be an interval representation of $G$. Let $C_{1}, \ldots, C_{s}$ be the sequence of all maximal cliques of $G$ listed from left to right according to $\mathcal{I}$. Let $L_{i}=C_{1} \cup \cdots \cup C_{i}$ for each $i \in[s]$. For any positive integer $k$, the graph $G$ has a spanning $k$-rail if and only if $G\left[L_{i}\right]$ has a typical $k$-fan with respect to $\mathcal{I}$ for every $i \in[s]$.

When $k=2$, Theorem 23 is already known by Keil and is useful in designing an efficient algorithm for deciding the Hamiltonicity of interval graphs; See Chen et al. (19, Lemma 2.3) and Keil (62).

Theorem 24 If $G$ is an interval graph, then $\left|\mathcal{H}_{G}(v)-\mathcal{H}_{G}(w)\right| \leq 1$ holds for all $v, w \in V(G)$.

Corollary 25 Let $G$ be an interval graph with $\mathcal{H}(G)=k$ and $|V(G)| \geq 2$. For every $v \in V(G)$, it holds $\mathcal{H}_{G}(v) \geq k-1$ and $G-v \in \digamma^{k-1}$.

Proof: It is a trivial consequence of Theorem 24

Lemma 26 Let $G$ be an interval graph with at least two vertices. Then $\min _{v \in V(G)} \mathcal{H}(G-v) \geq \mathcal{H}(G)-1$. 
Theorem 27 Let $G$ be a traceable interval graph with at least two vertices, namely an interval graph with Hamiltonian thickness at least 1 . Then

$$
\mathcal{H}(G)=\min _{v \in V(G)} \mathcal{H}(G-v)+1
$$

It also holds

$$
\mathcal{H}(G)=\min _{v \in V(G)} \mathcal{H}_{G}(v)+1
$$

unless $G$ is a complete graph.

Theorem 28 Let $G$ be an interval graph with at least two vertices. For every integer $k \geq 2$, the following hold.

a) The graph $G$ is a member of $\digamma^{k}$ if and only if $\mathcal{H}(G-v) \geq k-1$ for every $v \in V(G)$.

b) If $G$ is not a complete graph, then $G \in \digamma^{k}$ if and only if $\mathcal{H}_{G}(v) \geq k-1$ for every $v \in V(G)$.

For the graph $G=\overline{K_{3}}$ or $\overline{K_{2}}, \mathcal{H}_{G}(v)=\mathcal{H}(G)$ and $G-v \in \digamma^{0}$ for all $v \in V(G)$ but $G \notin \digamma^{1}$. This means that the condition of $\mathcal{H}(G) \geq 1$ in Theorem 27 and the condition of $k \geq 2$ in Theorem 28 are both necessary.

Theorem 29 Let $G$ be an interval graph. For any integer $k$ satisfying $|V(G)|>k \geq 3$, the following statements are equivalent.

i) The graph G lies in $\digamma^{k}$.

ii) For every nonnegative integer $s \leq k-3$ and every $S \in\left(\begin{array}{c}V(G) \\ s\end{array}\right)$, it holds $\operatorname{sfc}(G-S) \geq k-s-1$.

iii) The graph $G$ is spanning $(k-1)$-fan-connected.

iv) The graph $G$ is $(k-3)$-vertex-fault-tolerant Hamiltonian-connected.

For $k=2$, the next result on interval graphs basically follows from Theorem 5

Theorem 30 Let $G$ be an interval graph. For any given integers $k$ and $t$ such that $k \geq t \geq 2$, it holds $G \in \digamma^{k}$ if and only if $G$ is $(k-t)$-vertex-fault-tolerant spanning t-rail-connected. Especially, for any $k \geq 2, G \in \digamma^{k}$ if and only if $G$ is $(k-2)$-vertex-fault-tolerant Hamiltonian.

It looks much harder to obtain edge-fault-tolerance results based on an induction on the number of vertices. We mention that Lee and Park provided a good understanding of the vertex-fault-tolerant unpaired $k$-DPC problem on unit interval graphs but left its edge-fault-tolerant version as an open problem; See Lee and Park (71, p. 616). Luckily, we can get an edge version of Lemma 26, namely Theorem 31, from which Theorem 32, a result on the fault-tolerant Hamiltonian property, is only a few strides away.

Theorem 31 For every interval graph $G$ and $\mathcal{E} \subseteq E(G)$, it holds $\mathcal{H}(G-\mathcal{E}) \geq \mathcal{H}(G)-|\mathcal{E}|$.

Theorem 32 Let $G$ be an interval graph and let $k \geq 2$ be an integer. The graph $G$ lies in $\digamma^{k}$ if and only if it is $(k-2)$-fault-tolerant Hamiltonian. 
Given any nonnegative integer $k$, Theorems 30 and 32 imply that, for an interval graph, being $k$-faulttolerant Hamiltonian is equivalent to being $k$-vertex-fault-tolerant Hamiltonian. Its edge version is not correct. For example, we can consider the graph $G$ with vertex set $\left\{u, u^{\prime}, v_{1}, \ldots, v_{4 k+1}, w_{1}, \ldots, w_{4 k+1}\right\}$ and the interval representation $\mathcal{I}$ such that $\mathcal{I}(u)=\mathcal{I}\left(u^{\prime}\right)=[-1,1], \mathcal{I}\left(v_{1}\right)=\cdots=\mathcal{I}\left(v_{4 k+1}\right)=[-2,-1]$, and $\mathcal{I}\left(w_{1}\right)=\cdots=\mathcal{I}\left(w_{4 k+1}\right)=[1,2]$. Note that $G-u$ is not Hamiltonian but $G$ is $k$-edge-fault-tolerant Hamiltonian.

In some sense, Eq. (7) can be viewed as a dual of Eq. 6). Together, they strongly suggest the following result.

Theorem 33 For every interval graph $G$, it holds $\operatorname{sc}(G)+\mathcal{H}(G)=2$.

As an immediate application of Theorem 33 we can derive the following result of Broersma et al. (11).

Corollary 34 Let $G$ be an interval graph.

I) Broersma et al. (11. Theorem 2) For any positive integer $k, G$ has a typical k-rail if and only if $\operatorname{sc}(G) \leq 2-k$.

II) Broersma et al. (11] Theorem 3) For any integers $k<0, \operatorname{sc}(G)=k$ if and only if $G$ is $-(k+1)$ vertex-fault-tolerant Hamiltonian-connected but not $-k$-vertex-fault-tolerant Hamiltonian-connected.

Proof: I) Theorem 22 b) $\Leftrightarrow \mathrm{g}$ ) and Theorem 33 .

II) Theorems 29 i) $\Leftrightarrow$ iv) and Theorem 33

The join of two graphs $G_{1}$ and $G_{2}$, denoted by $G_{1} \bigvee G_{2}$, is the graph obtained from the disjoint union of $G_{1}$ and $G_{2}$, denoted by $G_{1} \cup G_{2}$, by adding all possible edges joining $V\left(G_{1}\right)$ and $V\left(G_{2}\right)$. Theorem 33 allows us obtain the following characterization of $\digamma^{k}$ for any nonpositive integer $k$.

Theorem 35 Let $G$ be an interval graph. For every integer $k \leq 0$, the following statements are equivalent.

a) The graph $G$ lies in $\digamma^{k}$.

b) $\pi(G) \leq 2-k$.

c) The graph $G \bigvee K_{1-k}$ lies in $\digamma^{1}$.

d) All normal vertex orderings of $G$ are $k$-thick Hamiltonian vertex orderings.

e) The graph $G$ has a normal $k$-thick Hamiltonian vertex ordering.

f) It holds $\operatorname{sc}(G) \leq 2-k$.

\subsubsection{Ordering constraints}

Let $G$ be a graph on $n$ vertices satisfying $\operatorname{Ore}^{\prime}$ s condition that $\operatorname{deg}_{G}(x)+\operatorname{deg}_{G}(y) \geq n$ for any nonadjacent pair of vertices $x$ and $y$. Chen et al. (18) found that such a graph is $k$-vertex-ordered Hamiltonian if either $k<\frac{n}{12}$ and $G$ is $\left\lceil\frac{k+1}{2}\right\rceil$-connected or $k \leq \frac{n}{176}$ and $G$ is $\left\lceil\frac{3 k}{2}\right\rceil$-connected. Eq. (3) suggests that there might be a counterpart of this result in terms of Hamiltonian thickness. The next result confirms this anticipation. 
Theorem 36 Let $k$ be a positive integer and let $G$ be an interval graph.

1) If $G$ has a $\left(\left\lfloor\frac{3 k}{2}\right\rfloor+2\right)$-thick Hamiltonian vertex ordering, then $G$ is $k$-ordered traceable.

2) If $G$ has a $\left(\left\lfloor\frac{3 k}{2}\right\rfloor+3\right)$-thick Hamiltonian vertex ordering, then $G$ is $k$-ordered Hamiltonian.

\subsubsection{Equivalences among various connectedness parameters}

Our first result is about the spanning rail connectivity and the spanning fan connectivity of interval graphs. Note that it is consistent with Eq. (5).

Theorem 37 Let $G$ be an interval graph.

i) If $|V(G)| \leq 3$, then $\operatorname{src}(G)=\operatorname{sfc}(G)$.

ii) If $\mathcal{H}(G) \leq 2$ and $|V(G)| \geq 4$, then $\operatorname{src}(G)=\operatorname{sfc}(G)=0$.

iii) If $\mathcal{H}(G) \geq 3$, then $\operatorname{src}(G)=\mathcal{H}(G)=\operatorname{sfc}(G)+1$.

A graph class is hereditary if it is closed under taking induced subgraphs. A hereditary graph class $\mathcal{C}$ is maroon provided, for every $G \in \mathcal{C}, G$ is connected if and only if $G$ is traceable.

Lemma 38 Let $\mathcal{C}$ be a maroon class of interval graphs. For every $G \in \mathcal{C}$, it holds

$$
\mathcal{H}(G)=\left\{\begin{aligned}
\kappa(G), & \text { if } \mathrm{c}(G)=1 \\
2-\mathrm{c}(G), & \text { if } \mathrm{c}(G)>1
\end{aligned}\right.
$$

A rigid chordal graph is a graph possessing a unique clique tree; See Ibarra (55), Li and Wu (73). A rigid chordal graph must be connected unless it is the disjoint union of two complete graphs. A rigid chordal graph is a rigid interval graph if it is also an interval graph; Li and Wu (73) studied rigid interval graphs with the help of graph search algorithms.

Lemma 39 A rigid interval graph is connected if and only if it is traceable.

Concerning the previous lemma, we remark that a connected interval graph is not necessarily traceable as can be seen from the claw graph $K_{1,3}$.

Theorem $40 \mathrm{Eq}$. 97 holds for every unit interval graph $G$.

Theorem 40 is a refinement of the known fact of Bertossi $(8)$ that every connected unit interval graph is traceable. We can also read from Theorems 29, 30 and 40 the known result of Chen and Chang (15), Chen et al. (17) that a unit interval graph is Hamiltonian if and only if it is 2-connected and a unit interval graph is Hamiltonian-connected if and only if it is 3-connected. The Matthews-Sumner Conjecture, as discussed in Broersma et al. (10), Matthews and Sumner (81), asserts that every 4-connected claw-free graph is Hamiltonian. Note that claw-free interval graphs are just unit interval graphs; See Roberts (94). Therefore, the fact that every 2-connected unit interval graph is Hamiltonian verifies the Matthews-Sumner Conjecture for interval graphs.

Let $G$ be a chordal graph which is not complete. Taking any clique tree $T$ of $G$, it is known that a set $S \subseteq V(G)$ is a minimal vertex separator of $G$ if and only if $S=C \cap C^{\prime}$ for two maximal cliques $C$ and 
$C^{\prime}$ of $G$ such that $C C^{\prime} \in E(T)$; See Ho and Lee (45). Especially, on the condition that the chordal graph $G$ is connected, we can derive that

$$
\kappa(G)=\min _{C C^{\prime} \in E(T)}\left|C \cap C^{\prime}\right| .
$$

Chen and Chang (15, Theorem 3) asserted without proof that $\kappa(G)=2-\operatorname{sc}(G)$ holds for every connected unit interval graph. The next result confirms that $\kappa(G), \operatorname{sc}(G)$ and several other parameters give the same information about a unit interval graph $G$.

Theorem 41 Let $G$ be a connected unit interval graph. Then $\kappa(G)=\mathcal{H}(G)=2-\operatorname{sc}(G)$. If $G$ has a clique tree $T$ with at least two vertices, it even holds $2 \mathrm{t}(G)=\kappa(G)=\mathcal{H}(G)=2-\operatorname{sc}(G)=$ $\min _{C C^{\prime} \in E(T)}\left|C \cap C^{\prime}\right|$.

Theorem 41 tells us that equality in Eq. (3) holds for all connected unit interval graphs $G$. We mention that equality in Eq. (4) does not necessarily hold for unit interval graphs $G$. To see this, we pick an integer $n>1$ and think of the graph $D_{n}$ obtained from two disjoint copies of $K_{n}$ by adding a universal vertex. It is easy to check that $D_{n}$ is a unit interval graph while $\operatorname{dgn}\left(D_{n}\right)=n>1=\kappa\left(D_{n}\right)$.

It is a well-established research direction to study the minimum number of edges to guarantee a Hamiltonian-connected graph. A classical result of Moon (84) says that the minimal number of edges in a Hamiltonian-connected graph with $n \geq 4$ vertices is $\left\lfloor\frac{3 n+1}{2}\right\rfloor$. Some recent studies can be found in Ho et al. (46).

Theorem 42 The minimum number of edges in a Hamiltonian-connected interval graph on $n \geq 3$ vertices is $3 n-6$, which is attained if and only if the interval graph is $K_{3}$ or an exact-3-thick Hamiltonian graph.

Theorem 43 Let $P$ be an interval poset and $G$ the cocomparability graph of $P$. The maximum Hamiltonian thickness of an ordering of $V(G)$ that is a linear extension of $P$ is equal to $\mathcal{H}(G)$.

\subsubsection{Extension properties}

For any graph $G$ with at least two vertices and $\pi \in V(G)$ !, we define

$$
f_{G}(\pi)=\left\{\begin{array}{c}
\min \left\{i: \mathrm{d}_{G, \pi}(i)=\mathcal{H}_{G}(\pi)\right\}, \text { if } \mathcal{H}_{G}(\pi)>0 \\
\max \left\{i: i<n, \mathrm{~d}_{G, \pi}(i)=0\right\}, \text { if } \mathcal{H}_{G}(\pi) \leq 0
\end{array}\right.
$$

For a graph $G$ with $\mathcal{H}(G) \geq 1$, Theorem 27 asserts the existence of a vertex $v$ such that $\mathcal{H}(G)=$ $\mathcal{H}(G-v)+1$. The next result beefs up this observation by pointing out how to get such a vertex $v$.

Theorem 44 Let $G$ be an interval graph with $\mathcal{H}(G)>0$. Let $\pi$ be any normal vertex ordering of $G$ and let $T=\left\{\pi_{j}: \pi_{j} \in N_{G}\left(\pi_{f_{G}(\pi)}\right), j>f_{G}(\pi)\right\}$. Then it holds $\mathcal{H}(G-S)=\mathcal{H}(G)-|S|$ for every $S \subseteq T$.

Theorem 45 For every integer $k \geq 2$ and every interval graph $G$, the following statements are equivalent.

i) The graph $G$ lies in $\digamma^{k}$.

ii) The graph $G$ is $(k-2)$-packable Hamiltonian.

iii) The graph $G$ is $(k-2)$-HC-extendable.

iv) The graph $G$ is strongly $(k-2)$-HP-extendable. 
v) The graph $G-S$ is traceable for every $S \in\left(\begin{array}{c}V(G) \\ \leq k-1\end{array}\right)$ such that $G[S]$ is a complete graph.

Observe that for $k=2$, Theorem $22 \mathrm{c}) \Leftrightarrow \mathrm{g}$ ) becomes Theorem $45 \mathrm{i}) \Leftrightarrow \mathrm{v}$ ).

Theorem 46 Let $k$ be a positive integer, let $G$ be an interval graph and let $S$ be a proper subset of $V(G)$. If both $G$ and $G[S]$ have typical k-rails, then there exists $x \in V(G) \backslash S$ such that $G[S \cup\{x\}]$ also has a typical $k$-rail.

In 1990, Hendry (44) conjectured that all chordal Hamiltonian graphs are cycle extendable, that is, the vertices of each non-Hamiltonian cycle are contained in a cycle of length one greater. Setting $k=1$ and 2 in Theorem 46, we derive the following two results from Theorem 22 .

Corollary 47 An interval graph is path extendable if and only if it is traceable.

Corollary 48 Abueida and Srithran (1. Theorem 4.2) Chen et al. (19. Theorem 1.2) An interval graph is cycle extendable if and only if it is Hamiltonian.

\subsubsection{Several linear time algorithms}

Theorem 49 Let $G$ be an interval graph. Then, $\mathcal{H}(G)$ equals to the Hamiltonian thickness of any normal vertex ordering of $G$ and hence can be computed in linear time.

Proof: Theorem $22 \mathrm{e}) \Leftrightarrow \mathrm{g}$ ) and Theorem $35 \mathrm{a}) \Leftrightarrow \mathrm{d})$.

Broersma et al. (11) posed the open problem of determining the computational complexity of calculating the spanning rail connectivity for interval graphs or even proper interval graphs. Our next result gives an answer to this problem.

Corollary 50 For every interval graph $G$, we can determine the parameters $\operatorname{src}(G)$ and $\operatorname{sfc}(G)$ in linear time.

Proof: According to Theorem 37, to determine $\operatorname{src}(G)$ and $\operatorname{sfc}(G)$, it suffices to compute $\mathcal{H}(G)$. It then follows from Theorem 49 that the computation of $\operatorname{src}(G)$ and $\operatorname{sfc}(G)$ can be done in linear time.

Very simple linear-time algorithm for computing the path cover number of an interval graph has been known; See Arikati and Pandu Rangan (3, Theorem 7) and Damaschke (29. Corollary 2). Recently, it is improved to be a certifying algorithm by Hung and Chang (54). Let us show that one can develop a linear time algorithm based on the concept of Hamiltonian thickness.

Corollary 51 Let $G$ be an interval graph and let $\pi$ be any normal vertex ordering of $G$. Then

$$
(\pi(G), \operatorname{sc}(G))=\left(2-\mathcal{H}_{G}(\pi), 2-\mathcal{H}_{G}(\pi)\right)
$$

if and only if $\mathcal{H}_{G}(\pi) \leq 0$, while

$$
(\pi(G), \operatorname{sc}(G))=\left(1,2-\mathcal{H}_{G}(\pi)\right)
$$

if and only if $\mathcal{H}_{G}(\pi)>0$. Therefore, $(\pi(G), \operatorname{sc}(G))$ can be determined in linear time. 
Proof: Eq. 22), Theorem 33 and Theorem 49

An $O\left(|V(G)|^{4}\right)$ time algorithm for calculating the scattering number of an interval graph $G$ was reported by Kratsch et al. (66) in 1994. In 2011, Hung and Chang (54) devised an $O(|E(G)|+|V(G)|)$ time algorithm to determine the scattering number of an interval graph $G$, on the condition that this scattering number is positive. In 2013, a linear time algorithm for getting both the scattering number and a scattering set of an interval graph was finally discovered by Broersma et al. (11, Corollary 2). Observe that our Corollary 51 already confirms that the scattering number of an interval graph can be calculated in linear time. Based on the concept of Hamiltonian thickness, the remainder of the present section will establish the correctness of the following algorithm (Algorithm 3.2) to determine a scattering set of an interval graph. Examining Algorithm 3.1 Algorithm 3.2 and Algorithm 3.3, we see easily that Algorithm $3.2(S C(G, \mathcal{I}, \pi))$ can be executed in $O(|V(G)|+|E(G)|)$ time.
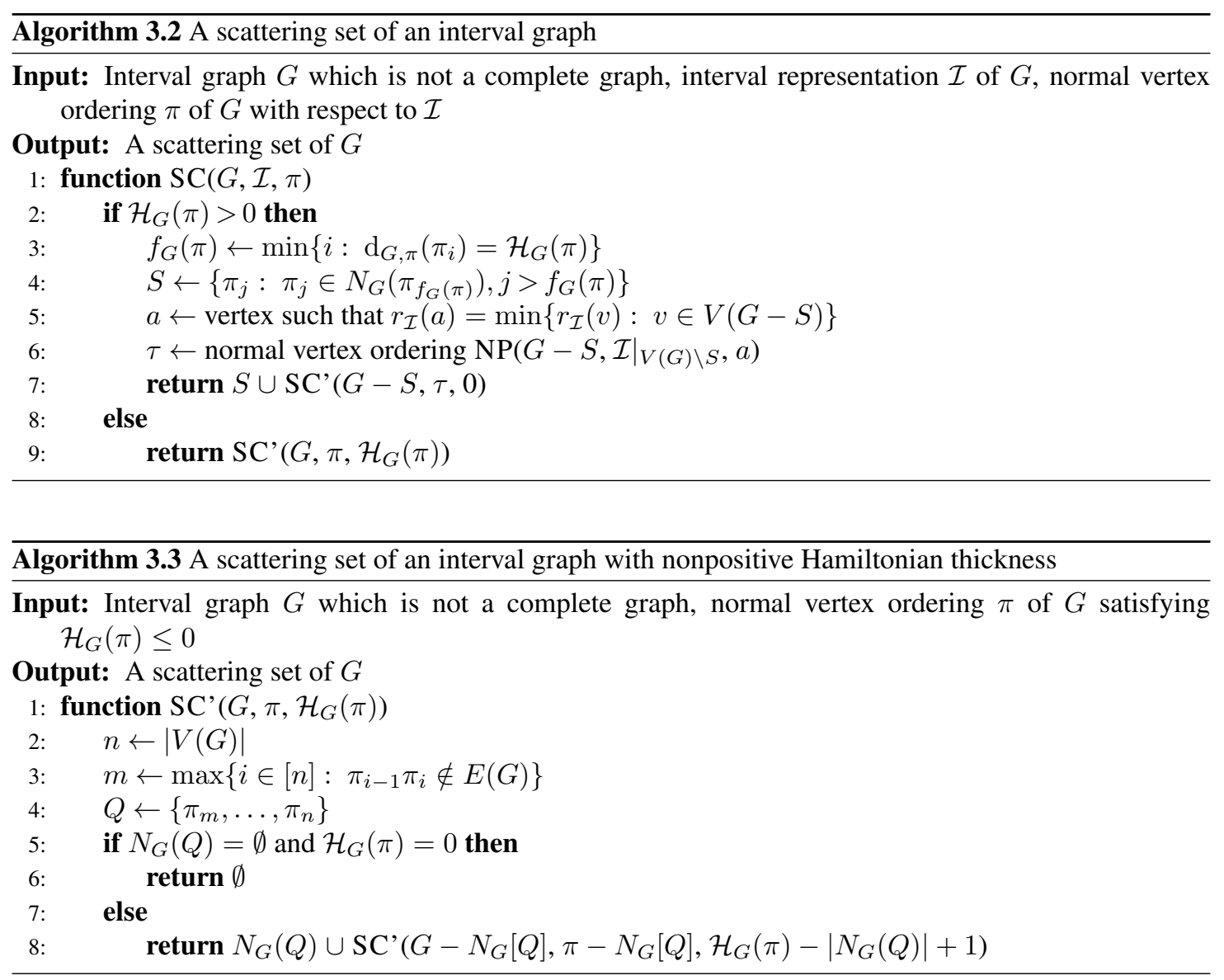

Let $S$ be a proper subset of $V$. For any permutation $\pi \in V$ !, let $\pi-S$ denote the element of $(V \backslash S)$ ! such that $x$ appears earlier than $y$ in $\pi$ if and only if $x$ appears earlier than $y$ in $\pi-S$ for every two elements $x, y \in V \backslash S$. 
Theorem 52 Let $G$ be an interval graph with $n \geq 2$ vertices and let $\pi$ be a normal vertex ordering of $G$. Let $m \in\{2, \ldots, n\}$ be an integer such that $\pi_{m-1} \pi_{m} \notin E(G)$ and let $Q=\left\{\pi_{m}, \ldots, \pi_{n}\right\}$.

(I) The ordering $\pi-N_{G}(Q)$ is a normal vertex ordering of $G-N_{G}(Q)$ and it holds $\mathcal{H}\left(G-N_{G}(Q)\right)=$ $\mathcal{H}(G)-\left|N_{G}(Q)\right|$;

(II) It holds $\pi_{m-1} \in V(G) \backslash N_{G}[Q]$. Moreover, the ordering $\pi-N_{G}[Q]$ is a normal vertex ordering of $G-N_{G}[Q]$ and $\mathcal{H}\left(G-N_{G}[Q]\right)=\mathcal{H}(G)-\left|N_{G}(Q)\right|+1+\left|\left\{t \in[m, n-1]: \pi_{t} \pi_{t+1} \notin E(G)\right\}\right|$.

Theorem 53 The algorithm $S C(G, \mathcal{I}, \pi)$ (Algorithm 3.2) is correct.

\subsubsection{Spanning version of the Chvátal-Erdős Theorem and the Menger's The- orem}

For a graph $G$, we use the notation $\alpha(G)$ for its independence number, namely the maximum size of an independent set of $G$. Chvátal and Erdős (24) opened a new vista for the study of Hamiltonian-type properties in 1972 by establishing the so-called Chvátal-Erdős Theorem: If $\alpha(G) \leq \kappa(G)-1$, then $G$ is Hamiltonian-connected; If $\alpha(G) \leq \kappa(G)$, then $G$ is Hamiltonian; If $\alpha(G) \leq \kappa(G)+1$, then $G$ is traceable. We try to give one possible spanning version of the Chvátal-Erdős Theorem for interval graphs.

Theorem 54 Let $G$ be an interval graph. Then $\mathcal{H}(G) \geq \kappa(G)-\alpha(G)+2$ if $G$ is not a complete graph and $\mathcal{H}(G)=\kappa(G)-\alpha(G)+1$ otherwise.

Let $G$ be a graph and let $v \in V(G)$. We write $\alpha_{G}(v)$ and $\kappa_{G}(v)$ for the independence number and the vertex connectivity, respectively, of the graph $G\left[N_{G}[v]\right]$. Aiming to get a localization version of the Chvátal-Erdős Theorem, Saito (98, Conjecture 5) conjectured that a connected graph $G$ with at least three vertices is Hamiltonian provided $\alpha_{G}(v) \leq \kappa_{G}(v)$ holds for each $v \in V(G)$. This conjecture was proved true by Oberly and Sumner $(86)$ when $\alpha_{G}(v) \leq 2 \leq \kappa_{G}(v)$ for each $v \in V(G)$. As implied by the next theorem, this conjecture also holds true for interval graphs.

Theorem 55 Let $G$ be a connected interval graph and $k$ be any nonnegative integer. If $\alpha_{G}(v) \leq \kappa_{G}(v)-$ $k$ holds for all $v \in V(G)$, then $G \in \digamma^{k+2}$ unless $G$ is a complete graph in which case we have $G \in \digamma^{k+1}$.

For a graph $G$, we have introduced the functions $p_{G}, c_{G}$ and $\widehat{c}_{G}^{1}$ in $\$ 2.1$ and $\$ 2.5$. The well-known Menger's Theorem, namely Bondy and Murty (9. Theorem 9.1), asserts that $c_{G}(u, v)=p_{G}(u, v)$ for any two nonadjacent vertices $u$ and $v$ in a graph $G$. Let us report below our effort of trying to deduce a spanning version of this Menger's Theorem for interval graphs.

Theorem 56 Let $G$ be an interval graph with $|V(G)|-1>\mathcal{H}(G) \geq 0$. For any two distinct vertices $u$ and $v$ in $G$, it holds $\widehat{c}_{G}^{1}(u, v) \leq \mathcal{H}(G)$.

Theorem 57 Let $G$ be an interval graph which is not any complete graph. For any two distinct vertices $u$ and $v$ in $G$, it holds

$$
\widehat{c}_{G}^{1}(u, v) \leq \widehat{p}_{G}(u, v)
$$

Example 58 Let $G$ be a graph with vertex set $\left\{v_{i}: i \in[7]\right\}$ and with an interval representation $\mathcal{I}$ such that $\mathcal{I}\left(v_{1}\right)=[1,3], \mathcal{I}\left(v_{2}\right)=[2,5], \mathcal{I}\left(v_{3}\right)=\mathcal{I}\left(v_{4}\right)=[4,7], \mathcal{I}\left(v_{5}\right)=[6,9], \mathcal{I}\left(v_{6}\right)=[8,10], \mathcal{I}\left(v_{7}\right)=$ $[1,10]$. Then we see that

$$
\left(v_{2}, v_{1}, v_{7}, v_{6}, v_{5}\right),\left(v_{2}, v_{3}, v_{5}\right),\left(v_{2}, v_{4}, v_{5}\right)
$$

form a spanning 3-rail between $v_{2}$ and $v_{5}$ in $G$. But it is clear that $G-v_{7}$ does not have any spanning rail between $v_{2}$ and $v_{5}$, hence $1=\widehat{c}_{G}^{1}\left(v_{2}, v_{5}\right)<\widehat{p}_{G}\left(v_{2}, v_{5}\right)=3$. 
Example 59 Take an integer $n \geq 4$. Let $G=\left(K_{1, n} \cup K_{1, n}\right) \bigvee K_{n}$ and let $u$ and $v$ be the only two vertices whose degree is $2 n$ in $G$. Then $G$ is an interval graph which is not traceable and has local spanning rail connectivity $\widehat{p}_{G}(u, v)=n$. This example also says that there exist nontraceable graphs with arbitrarily high vertex connectivity and arbitrarily high local spanning rail connectivity.

Example 60 (Xuding Zhu) Let $G$ be the Coxeter graph as defined in Bondy and Murty (9), Coxeter (28). Let $u$ and $v$ be two vertices whose distance in $G$ is 3 or 4 . Then $\widehat{c}_{G}^{1}(u, v) \geq 2>\widehat{p}_{G}(u, v)$.

\subsubsection{Graph power and traceable number}

Let $G$ be a connected graph. For any $u, v \in V(G)$, $\operatorname{dist}_{G}(u, v)$ denotes the distance between $u$ and $v$ in $G$. For any positive integer $k$, we define the $k$ th power of $G$, denoted by $G^{k}$, to be the graph with $V\left(G^{k}\right)=V(G)$ and $E\left(G^{k}\right)=\left\{u v: 1 \leq \operatorname{dist}_{G}(u, v) \leq k\right\}$. For any word $\pi=\pi_{1} \cdots \pi_{n}$ over $V(G)$, let $\operatorname{dist}_{G}(\pi)=\sum_{i \in[n-1]} \operatorname{dist}_{G}\left(\pi_{i}, \pi_{i+1}\right)$. Following Saenpholphat et al. (97), the traceable number of $G$, denoted as $\operatorname{trn}(G)$, is defined to be

$$
\min \left\{\operatorname{dist}_{G}(\pi): \pi \text { is an ordering of } V(G)\right\} .
$$

A trivial observation is that $\operatorname{trn}(G) \leq(|V(G)|-1) k$ provided $G^{k}$ is traceable.

It is natural to expect that sufficiently large power of a graph will have strong spanning connectedness property. A classical result says that the cube of every connected graph is Hamiltonian-connected; See Karaganis (61), Sekanina (100). In 2013, Park and Ihm (90, Theorem 2) showed that the cube of a connected graph with at least four vertices is spanning 3-rail-connected; More generally, Sabir and Vumar (96. Theorem 5) proved that if a connected graph $G$ has at least $k+1 \geq 4$ vertices then $G^{k}$ is spanning $k$-rail-connected. It is also noteworthy that Park and Ihm (90, Theorem 1) characterized those connected graphs $G$ for which $G^{3}$ are spanning 3-fan-connected. Recall that the powers of an interval graph are still interval graphs; See Raychaudhuri (93). Thus, when restricting to interval graphs, an application of Theorem 30 gives the interpretation of the Sabir-Vumar Theorem (96, Theorem 5) that $G^{k} \in \digamma^{k}$ for any connected interval graph $G$ with at least $k+1 \geq 4$ vertices. Since we have shown that high Hamiltonian thickness will guarantee various spanning connectedness properties, we will follow this line of research by developing lower bounds of the Hamiltonian thickness of graph powers, including a generalization of the aforementioned interpretation of the Sabir-Vumar Theorem for interval graphs (Corollary 67).

Following Corneil et al. (26, Definition 1.2), for any graph $G$, we call an element $\pi \in V(G)$ ! a path ordering of $G$ provided for every three vertices $\pi_{i}, \pi_{j}$ and $\pi_{k}$ with $1 \leq i<j<k \leq|V(G)|$, it holds either $\pi_{i} \pi_{k} \in E(G)$ or $\pi_{i}$ and $\pi_{k}$ fall in different components of $G-N_{G}\left[\pi_{j}\right]$. Corneil et al. (26, Definition 1.2) named a graph possessing a path ordering a path orderable graph. Corneil et al. (26, p. 106) pointed out that all cocomparability graphs are path orderable.

Theorem 61 Let $G$ be a connected graph with a path ordering $\pi$. Then $\pi$ is a Hamiltonian vertex ordering of $G^{3}$.

Theorem 62 Let $k$ and $p$ be two positive integers and let $G$ be a p-connected path orderable graph. If $k \geq 4$ and $G$ has at least $(k-1) p+1$ vertices, then it holds $G^{k} \in \digamma^{(k-1) p}$. If $k=3$ and $G$ has at least $(k-2) p+1=p+1$ vertices, then it holds $G^{k}=G^{3} \in \digamma^{p}$.

Let $G$ be a graph with $n$ vertices and let $\pi=\pi_{1} \cdots \pi_{n}$ be an ordering of all the vertices of $G$. We call this ordering $\pi$ a $k$-good vertex ordering of $G$ provided the following hold: 
- $n \geq k+1$;

- $\operatorname{dist}_{G}\left(\pi_{i}, \pi_{i+1}\right) \leq k$ and $G\left[\pi_{i}, \ldots, \pi_{n}\right]$ is connected for each $i \in[n-k]$.

Let $(P,<)$ be a poset. For any $x \in P$, let $x \uparrow_{P}$ denote the set $\{y \in P: x<y\}$. An element $x \in P$ is called a bottom element of $P$ if the set $x \uparrow_{P}$ has the maximum possible size. Note that a bottom element must be a minimal element but the converse may not be true. Let $\pi=\pi_{1} \cdots \pi_{n}$ be an ordering of the elements of $P$. We call $\pi$ an ordinary ordering provided $\pi_{i}$ is a bottom element in the subposet induced by $\left\{\pi_{i}, \ldots, \pi_{n}\right\}$ for every $i \in[n-1]$.

Theorem 63 Let $\pi$ be an ordinary ordering of a poset $P$ with $n$ elements. Let $G$ be the cocomparability graph of $P$. If $G$ is connected and $n \geq 3$, then $\pi$ is a 2-good vertex ordering of $G$.

Theorem 64 Let $k$ and $p$ be two positive integers. If $k \geq 2$, then for every $p$-connected cocomparability graph $G$ with at least $(k-1) p+1$ vertices, it holds $G^{k} \in \digamma^{(k-1) p}$.

For any connected graph $G$ on $n$ vertices and any $s \in[n-1]$, we define the $s$-thickness exponent of $G$, denoted by $\exp _{\mathcal{H}}(G, s)$, to be the minimum positive integer $k$ such that $G^{k} \in \digamma^{s}$.

Example 65 Let $m$ be an integer greater than 4 . Let $G$ be the graph with $V(G)=\left\{x, s_{1}, t_{1}, \ldots, s_{m}, t_{m}\right\}$ and $E(G)=\left\{x s_{i}, s_{i} t_{i}: i \in[m]\right\}$. It is not hard to check that $G^{2} \notin \digamma^{1}$ and $G^{3} \in \digamma^{1}$. This means that $\exp _{\mathcal{H}}(G, 1)=3$. This also illustrates that Theorem 64 cannot be extended to all graphs.

Theorem 66 Let $G$ be a graph and $k$ a positive integer. It holds $G^{k} \in \digamma^{k}$ as long as $G$ has a $k$-good vertex ordering.

Corollary 67 Let $k$ be an integer not less than 2 . Let $G$ be a connected cocomparability graph with at least $k+1$ vertices. Then $G^{k} \in \digamma^{k}$.

Proof: Follows from Theorems 63 and 66

Theorem 68 Let $k$ and $p$ be two positive integers. If $k \geq 2$, then for every $p$-connected interval graph $G$ with at least $k p+1$ vertices, it holds $G^{k} \in \digamma^{k p}$.

Corollary 69 Let $k$ and $p$ be two positive integers. For every p-connected unit interval graph $G$ with at least $k p+1$ vertices, it holds $G^{k} \in \digamma^{k p}$.

Proof: Theorems 40 and 68

Corneil et al. (26) showed that

$\{$ cocomparability graphs $\} \subsetneq\{$ path orderable graphs $\} \subsetneq\{$ AT-free graphs $\}$.

When restricted to cocomparability graphs, Theorem 64 says that the Hamiltonian thickness bound $(k-$ 1) $p$ in Theorem 62 applies not only for $k \geq 4$ but for all $k \geq 2$. When restricted to cocomparability graphs and $p=1$, Corollary 67 strengthens Theorem 62 by replacing $\digamma^{k-1}$ with $\digamma^{k}$. When further restricted to interval graphs and $k \geq 2$, Theorem 68 improves Theorem 62 by substituting $\digamma^{(k-1) p}$ by $\digamma^{k p}$. Finally, when considering merely unit interval graphs, Corollary 69 goes one step ahead from Theorem 68 by including the case of $k=1$.

Let us present an estimate of $\operatorname{trn}(G)$ in terms of $\mathcal{H}(G)$. 
Theorem 70 Let $G$ be a connected interval graph with $n \geq 2$ vertices. Then it holds $n-1+\beta \leq$ $\operatorname{trn}(G) \leq n-1+2 \beta$ where $\beta=\max \{0,1-\mathcal{H}(G)\}$.

Example 71 Consider the following set of intervals on the real line: $\mathcal{I}(1)=[1,2], \mathcal{I}(2)=[1,6], \mathcal{I}(3)=$ $[3,4], \mathcal{I}(4)=[7,9], \mathcal{I}(5)=[8,11], \mathcal{I}(6)=[10,12], \mathcal{I}(7)=[5,12]$. They correspond to the interval graph $G$ with $V(G)=[7]$ and $E(G)=\{12,23,27,45,47,56,57,67\}$. It is easy to see that both 1234567 and 6547231 are normal vertex orderings of $G$, for which we have $\operatorname{trn}(G)=\operatorname{dist}_{G}(6547231)=7<$ $8=\operatorname{dist}_{G}(1234567)$.

\subsection{Further research}

Question 72 Theorems 5,7 and 9 are three results on "vertex-super" properties. Will the results still hold true when we replace "vertex-super" by "super"? To tackle this question requires us consider not only the vertex constraints but also the edge constraints. It may bring us up to an entirely new difficulty level, as can be seen from our proof of Theorem 32.

Question 73 It is well-known that the chromatic number $\chi(G)$ of a graph $G$ is at most $\operatorname{dgn}(G)+1$. What are those graphs $G$ satisfying $\chi(G) \leq \mathcal{H}(G)+1$ ? By Theorem 18 . exact-k-thick Hamiltonian graphs are among those graphs.

When restricted to interval graphs, the next conjecture is confirmed by the equivalence of a) and d) in Theorem 22 (also by Theorems 16 and 33). Theorem 19 may be counted as another minor step towards a possible proof of Conjecture 74 Note that the graph $G_{k}$ in Example 20 f) is chordal and so we could not expect a proof of Conjecture 74 via a straightforward application of Theorem 16.

Conjecture 74 The class of chordal graphs have the Deogun-Kratsch-Steiner property.

Theorem 19 and the $\mathrm{c}) \Leftrightarrow \mathrm{d}$ ) implication in Theorem 22 suggest the next question.

Question 75 For which natural graph class $\mathscr{G}$ is the following statement true? Take any $G \in \mathscr{G}$ and any positive integer $k$ smaller than $|V(G)|-1$, the deletion of any $k$ vertices from $G$ always results in a traceable graph if and only if the deletion of at most $k$ vertices from $G$ always results in a traceable graph. Will it hold when $\mathscr{G}$ is the class of cocomparability graphs?

Results like Theorems 21, 27, 31, 44 and 52 should be useful for an induction approach to establish various spanning connectedness properties of graphs with sufficiently high Hamiltonian thickness. With this in mind, we put forward the following sequence of conjectures in Conjecture 76. In $\$ 3.1 .2$, we indicate that there may exist close relationship between Hamiltonian thickness and scattering number and so our next conjecture is also a pursuit of some counterpart for Eq. (6).

Conjecture 76 Let $G$ be a graph.

a) For every $v \in V(G)$, it holds $\mathcal{H}(G) \leq \mathcal{H}(G-v)+1$.

b) The set $\left\{\mathcal{H}_{G}(v): v \in V(G)\right\}$ consists of a set of consecutive integers.

c) For every $u v \in E(G)$, it holds $\mathcal{H}_{G}(v) \leq \mathcal{H}_{G}(u)+1$.

d) For every $\{u, v\} \in\left(\begin{array}{c}V(G) \\ 2\end{array}\right)$, it holds $\mathcal{H}_{G}(u) \leq \mathcal{H}_{G-v}(u)+1$.

For the four statements in Conjecture 76, we can easily see the following implications: 

a) $\Leftarrow$
d) $\Rightarrow$
c) $\Rightarrow$
b)

Ziqing Xiang verified Conjecture 76 d) with computer for all graphs with at most nine vertices.

We call a graph $G$ flat provided $\mathcal{H}_{G}(v) \in\{\mathcal{H}(G), \mathcal{H}(G)-1\}$ for every $v \in V(G)$. Observe that all flat graphs satisfy Conjecture 76 a). Theorem 21 a) says that all graphs $G$ with $\mathcal{H}(G) \leq 2$ are flat. In addition, Theorem 24 says that all interval graphs are flat. Ziqing Xiang checked via computer experiment that all graphs with at most eight vertices are all flat. However, he also found that there are exactly thirteen graphs on nine vertices which are not flat and even constructed an infinite family of graphs which are not flat (Example 77).

Example 77 (Ziqing Xiang) Let $n \geq 8$. Let $H_{n}$ be the graph with $V\left(H_{n}\right)=\left\{v_{i}: i \in[n-1] \cup\{0\}\right\}$ and $E\left(H_{n}\right)=E_{1} \cup E_{2} \cup E_{3} \cup E_{4}$ where $E_{1}=\left\{v_{0} v_{1}, \ldots, v_{n-2} v_{n-1}, v_{n-1} v_{0}\right\}, E_{2}=\left\{v_{2} v_{4}, v_{3} v_{5}\right.$, $\left.\ldots, v_{n-5} v_{n-3}\right\}, E_{3}=\left\{v_{n-3} v_{n-1}, v_{n-1} v_{1}\right\}$ and $E_{4}=\left\{v_{n-4} v_{n-1}, v_{n-3} v_{0}\right\}$. Then $\mathcal{H}_{H_{n}}\left(v_{0}\right)=2$ and $\mathcal{H}_{H_{n}}\left(v_{i}\right)=1$ for $i \in[n-1]$. Let $\widehat{H}_{n+1}$ be the graph obtained from $H_{n}$ by adding a new vertex $v_{n}$ and the set of new edges $\left\{v_{n} v_{i}: i \in[n-1]\right\}$. Then $\mathcal{H}_{\widehat{H}_{n+1}}\left(v_{0}\right)=3, \mathcal{H}_{\widehat{H}_{n+1}}\left(v_{n}\right)=1$ and $\mathcal{H}_{\widehat{H}_{n+1}}\left(v_{i}\right)=2$ for $i \in[n-1]$. See Fig. 4 for the graphs $H_{8}$ and $\widehat{H_{9}}$.

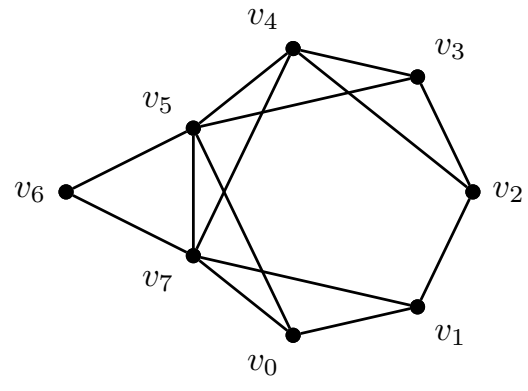

(a) $H_{8}$

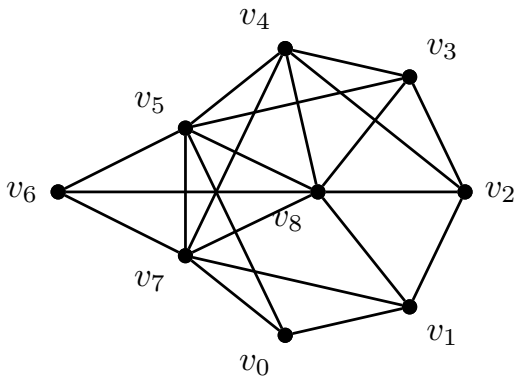

(b) $\widehat{H}_{9}$

Fig. 4: The graph $H_{8}$ is flat while the graph $\widehat{H}_{9}$ is not flat.

Question 78 Provide sufficient or necessary conditions for a (non-vertex-transitive) graph to be flat.

Let $G$ be an $n$-vertex graph, let $S$ and $T$ be two disjoint subsets of $V(G)$, and let $w \in V(G) \backslash(S \cup T)$. For any $\mu \in S$ ! and $\tau \in T$ !, let $\mathcal{H}_{G}(\mu ; \tau)$ denote

$$
\max \left\{\mathcal{H}_{G}(\pi): \pi \in V(G) !, \pi[1,|S|]=\mu, \pi[n-|T|+1, n]=\tau\right\}
$$

Let $\epsilon$ denote the empty word, namely the word of length 0 . Observe that $\mathcal{H}_{G}(\mu ; \epsilon)=\mathcal{H}_{G}\left(\mu_{1}\right)$ whenever $\mu=\mu_{1}$ is a length one word. To tackle Conjecture 76 or Question 78, and more generally to understand the Hamiltonian thickness of graphs, it may be of interest to examine the relationship between $\mathcal{H}_{G}(\mu ; \tau)$ and $\mathcal{H}_{G-w}(\mu ; \tau)$

Example 79 Let $G$ be the diamond graph depicted in Fig. 5 Unlike those statements in Conjecture 76. it does not hold $\mathcal{H}_{G}\left(v_{1} ; v_{4}\right) \leq \mathcal{H}_{G-v_{2}}\left(v_{1} ; v_{4}\right)+1$, as it occurs $\mathcal{H}_{G}\left(v_{1} ; v_{4}\right)=2$ and $\mathcal{H}_{G-v_{2}}\left(v_{1} ; v_{4}\right)=0$. 


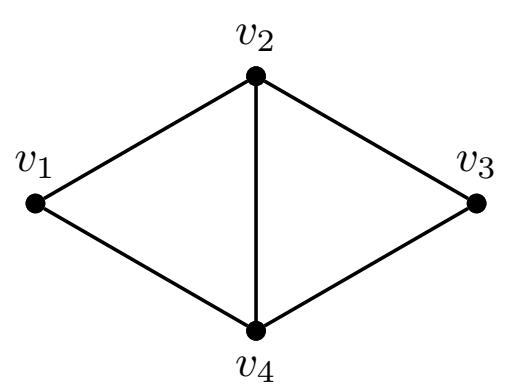

Fig. 5: The diamond.

Question 80 Are there counterparts of Theorems 22, 29, 30, or 35for strongly chordal graphs?

Theorem 36 may be strengthened as follows.

Conjecture 81 Let $G$ be a chordal graph and let $k$ be a positive integer. If $G$ has a k-thick Hamiltonian vertex ordering, then $G$ is $k$-ordered Hamiltonian.

It is an easy observation of Lin et al. (78, Lemma 1) that a graph with at least three vertices is spanning 1 -fan-connected if and only if it is spanning 2 -fan-connected. More generally, we have the next conjecture, whose truth for interval graphs is guaranteed by Theorem 37

Conjecture 82 Let $k$ be a positive integer. i) If $G$ is spanning $(k+1)$-fan-connected, then it must be spanning $k$-fan-connected. ii) If $G$ is spanning $(k+2)$-rail-connected, then it must be spanning $(k+1)$ rail-connected.

The bump number of a poset $P$ is an invariant of its cocomparability graph; Indeed, Damaschke et al. (30, Theorem 2, Theorem 3) proved that it is equal to the path cover number of its cocomparability graph. This fact together with Theorem 43 suggests the next question.

Question 83 For a poset $P$ and its cocomparability graph $G$, is the maximum thickness of a linear extension of $P$ a graph invariant? What is its relationship with $\mathcal{H}(G)$ ?

The following conjecture is a strengthening of Theorem 46 .

Conjecture 84 Given any positive integer $k$, every interval graph $G \in \digamma^{k}$ is $k$-rail extendable.

In $\$ 3.2 .5$, we mention a conjecture of Hendry (44) on chordal Hamiltonian graphs. Corollary 47 there hints at the following variant of that conjecture.

Conjecture 85 All chordal traceable graphs are path extendable.

Conjecture 86 Let $G$ be a connected graph such that $G^{2}$ is Hamiltonian. Then it holds $G^{2} \in \digamma^{2}$.

For any graph $G$, Chvátal (23) found that $\mathrm{t}(G) \geq \frac{\kappa(G)}{\alpha(G)}$ and equality holds when $G$ is a complete bipartite graph. Combined with Theorems 1 and 54 , this leads to the classical result that an interval graph with at least three vertices is Hamiltonian if and only if it is 1-tough; See Keil (62) and Chen et al. (19) Lemma 2.4). Deogun et al. (32, Theorem 10) strengthened this classical result by proving that a 
cocomparability graph with at least three vertices is 1-tough if and only if it is Hamiltonian. The next conjecture, if proved true, can provide a new proof of this result of Deogun et al.

Conjecture 87 Theorem 54 remains true if we allow the graph $G$ to be a general cocomparability graph.

Question 88 Is Theorem 55 still true when the parameter $k$ takes negative value?

Theorem 55 says that the following conjecture holds for interval graphs.

Conjecture 89 Let $G$ be a connected graph and $k$ be a nonnegative integer. If $\alpha_{G}(v) \leq \kappa_{G}(v)-k$ holds for each $v \in V(G)$, then $G \in \digamma^{k+2}$ unless $G$ is a complete graph in which case we have $G \in \digamma^{k+1}$.

The next conjecture is motivated by Theorem 57

Conjecture 90 Let $G$ be an interval graph. For any two nonadjacent vertices $u$ and $v$ in $G$, it holds $\widehat{c}_{G}(u, v) \leq \widehat{p}_{G}(u, v)$.

Question 91 Bauer et al. (7) studied sufficient conditions on the vertex degrees for guaranteeing a $t$ tough graph. Can we replace degree sequences by forward degree sequences and deduce corresponding results? Especially, for claw-free graphs, is there any relationship between the Hamiltonian thickness and the toughness?

Theorem 64 and Example 65 together leads us to the next question.

Question 92 Let $p$ be a positive integer, let $k$ be an integer not less than 3 and let $n$ be an integer not less than $(k-1) p+1$. Let $G$ be a $p$-connected graph on $n$ vertices. Is it always true that $G^{k} \in \digamma^{(k-1) p}$ ? For $p=1$, we can establish this inequality and will report it among other results elsewhere.

In view of Theorem 66, to deduce the spanning connectedness of graph powers in terms of the Hamiltonian thickness parameter, it may be useful if the following conjecture can be proved true.

Conjecture 93 Every 2-connected graph has a 2-good vertex ordering.

For every integer $p \geq 2$ and every AT-free graph $G$, Chang et al. (13, Theorem 4) found that $G^{p}$ is a cocomparability graph. As a consequence of Corollary 67, we then see that for any integers $k$ and $p$ greater than or equal to 2, it holds $G^{k p} \in \digamma^{k}$ for every connected AT-free graph $G$ with at least $k+1$ vertices. Recall Eq. (13).

Question 94 Can we deduce lower bounds of the Hamiltonian thickness of powers of AT-free graphs which, when restricted to path orderable graphs, is no worse than the bound in Theorem 62.?

Conjecture 95 Let $G$ be an interval graph with $\mathcal{H}(G) \geq 4$. Let $x$ and $y$ be two different vertices in $G$ which are of distance $d$ apart. For each integer $i \in\left[d, \frac{|V(G)|}{2}\right]$, there is a Hamiltonian cycle of $G$ such that the distance between $x$ and $y$ along $C$ is exactly $i$.

Question 96 Let $G$ be a graph which is not complete and let $\lambda(G)=\min _{\{u, v\} \in\left(\begin{array}{c}V_{2}(G) \\ (G)\end{array}\right.}\left(\operatorname{deg}_{G}(u)+\right.$ $\left.\operatorname{deg}_{G}(v)-|V(G)|+2\right)$. When $G$ is an interval graph or when $\lambda(G) \leq 1$, one can check that $G \in \digamma^{\lambda(G)}$ holds. For integers $n \geq 2$ and $t \geq 1$, if $G$ is the complete multipartite graph $K_{t ; n}$ (Example 8), we know that $\mathcal{H}(G)=n-1<(n-2) t+2=\lambda(G)$. Can we characterize those graphs $G$ for which $\mathcal{H}(G) \geq \lambda(G)$ holds? 
We have seen as above the usefulness of the concept of Hamiltonian thickness in getting new results and motivating new problems on spanning connectedness. We believe that much work lies ahead along this direction and the concept of Hamiltonian thickness will continue to intrigue and inspire. Especially, degree sequence appears in much wider research field and we wonder if one can try to replace degree sequence by forward degree sequence with the aid of a good search (vertex ordering) strategy and thus open up some hidden doors to more mathematical surprises.

\section{Proofs}

Let $G$ be a graph, $P=\left(x_{1}, \ldots, x_{s}\right)$ and $Q=\left(y_{1}, \ldots, y_{t}\right)$ be two vertex-disjoint paths of $G$. If $x_{1} x_{s} \in$ $E(G)$, we write $\langle P\rangle$ for the cycle $\left\langle x_{1}, \ldots, x_{s}\right\rangle$. If $P$ and $Q$ are vertex disjoint and $x_{s} y_{1} \in E(G)$, we call $\left(x_{1}, \ldots, x_{s}, y_{1}, \ldots, y_{t}\right)$ the path obtained from $P$ and $Q$ by connecting $x_{s}$ and $y_{1}$ and denote it by $P x_{s} y_{1} Q$. If $x_{s}=y_{1}=a$ and $V(P) \cap V(Q)=\{a\}$, we designate the path $\left(x_{1}, \ldots, x_{s}, y_{2}, \ldots, y_{t}\right)$ by $P \underline{a} Q$ and refer to it as the path obtained by connecting $P$ and $Q$ at $a$. We sometimes simply put $P Q$ to represent either $P \underline{x_{s} y_{1}} Q$ or $P \underline{a} Q$ if it is clear from the context.

\subsection{General graphs}

\subsubsection{Proofs of Theorems $1,5,7$ and 9}

Proof of Theorem 1; We may assume that $V(G)=[n]$ and $\pi_{i}=i$ for all $i \in[n]$. For any number sequence $P$ we use $\max P$ to denote the largest element in the sequence. We will define $k$ increasing sequences $P_{1}(t), \ldots, P_{k}(t)$ inductively from $t=1$ till step $t=s$ so that, for all $j \in[k]$, the last term in the increasing sequence $P_{j}(s)$ equals $n$, namely $\max P_{j}(s)=n$. Indeed, we can think that we have $k$ paths $P_{1}, \ldots, P_{k}$ which are growing as time $t$ evolves. We start from $P_{1}(1)=\cdots=P_{k}(1)=1$. Suppose that $P_{1}(t), \ldots, P_{k}(t)$ have been defined, $\min \left\{\max P_{j}(t): j \in[k]\right\}<n$ and $i$ is the smallest number such that $\max P_{i}(t)=\min \left\{\max P_{j}(t): j \in[k]\right\}$. We put $P_{j}(t+1)=P_{j}(t)$ for $j \neq i$. If there exists $q \in[n] \backslash\left(\cup_{j=1}^{k} P_{j}\right)$ such that $q \max P_{i}(t) \in E(G)$ and $\max P_{i}(t)<q$, we take the smallest such $q$ and add it to the end of the sequence $P_{i}(t)$ to produce a new increasing sequence $P_{i}(t+1)$. If no such $q$ exists, we add $n$ to the end of the sequence $P_{i}(t)$ to construct $P_{i}(t+1)$. We continue this process of growing the sequences $P_{1}, \ldots, P_{k}$ and finally arrive at $P_{1}(s), \ldots, P_{k}(s)$ for some $s$ such that $\max P_{j}(s)=n$ for all $j \in[k]$.

To complete the proof, it is enough to verify that $P_{1}(s), \ldots, P_{k}(s)$ form a spanning $k$-rail between $1=\pi_{1}$ and $n=\pi_{n}$. Indeed, since every path in this spanning $k$-rail is an increasing sequence, $\pi_{n-1}$ must be adjacent to $\pi_{n}$ on one of the $k$ paths in the asserted spanning $k$-rail.

Note that a consequence of $\mathcal{H}(G) \geq k>0$ is that $n \geq k+1$. This means that the set of second vertices on $P_{1}(s), \ldots, P_{k}(s)$ are exactly the smallest $k$ neighbors of 1 in $G$ and so $P_{1}(s), \ldots, P_{k}(s)$ must be $k$ different sequences.

We continue to show that $P_{1}(s), \ldots, P_{k}(s)$ form a $k$-rail between $1=\pi_{1}$ and $n=\pi_{n}$. For this purpose, we arbitrarily pick $j \in[k]$ and suppose that $P_{j}(s)=\left(v_{1}, \ldots, v_{m}\right)$ where $v_{1}=1$ and $v_{m}=n$. Our task is to prove that $P_{j}(s)$ is a path, or, equivalently, $v_{m-1} v_{m}=v_{m-1} n \in E(G)$. If $v_{m-1} \geq n-k$, it follows from $\mathcal{H}_{G}(\pi) \geq k$ that $v_{m-1} n \in E(G)$. Now consider the case that $v_{m-1}<n-k$, for which, in view of $\mathcal{H}_{G}(\pi) \geq k$, we have $\mathrm{d}_{G, \pi}\left(v_{m-1}\right) \geq k$. Take the integer $t$ such that $n \in P_{j}(t) \backslash P_{j}(t-1)$; Surely we have $t \leq s$. If $v_{m-1} n \notin E(G)$, the pigeonhole principle implies that there exists $\ell \in[k] \backslash\{j\}$ such that $N_{G}\left(v_{m-1}\right) \cap\left\{v_{m-1}+1, \ldots, n\right\} \cap P_{\ell}(t)$ contains two different vertices $x$ and $y$. We assume that $x<y$ and 
let $r_{1}<r_{2}$ be the two integers less than $t$ such that $x \in P_{\ell}\left(r_{1}\right) \backslash P_{\ell}\left(r_{1}-1\right)$ and $y \in P_{\ell}\left(r_{2}\right) \backslash P_{\ell}\left(r_{2}-1\right)$. By virtue of $x>v_{m-1}$, we know from the rule of growing paths that $P_{\ell}$ will stand still from time $r_{1}$ till the time $t$ when $P_{j}$ will grow in next step from $v_{m-1}$ to $n$. This contradicts the fact that $y \in P_{\ell}\left(r_{2}\right) \backslash P_{\ell}\left(r_{1}\right)$ and $r_{1}<r_{2}<t$.

We are left with the task of demonstrating that every $q \in[n]$ appears in one of the paths $P_{1}(s), \ldots, P_{k}(s)$. If this were not true, let $q$ be the minimum integer in $[n]$ which does not belong to $\cup_{j=1}^{k} P_{j}(s)$. Clearly, $q>1$. This means that there is an $\ell$ such that $q-1 \in P_{\ell}(s)$ and then we can infer from $q \notin \cup_{j \neq \ell} P_{j}(s)$ that the next number in the increasing sequence $P_{\ell}(s)$ must be $q$, which violates our assumption.

Lemma 97 Let $G$ be a graph with a 2-thick Hamiltonian vertex ordering $\pi_{1} \cdots \pi_{n}$. For any $i \in[n-1]$, there are a $j \in[i]$ and a spanning $\pi_{i}, \pi_{j}$-path of $G\left[\pi_{1}, \ldots, \pi_{i}\right]$ such that $[i] \cap\left\{k: k>j+1, \pi_{k} \pi_{j} \in\right.$ $E(G)\}=\emptyset$.

Proof: If $i=1$, then we can surely pick $j=1$. We then assume that $1<i<n$. Because $\pi$ is 2-thick, there is a sequence $\alpha_{0}-1=1<\alpha_{0}=2 \leq \alpha_{1}-1<\alpha_{1} \leq \alpha_{2}-1<\alpha_{2} \leq \alpha_{3}-1<\alpha_{3}<\cdots \leq$ $\alpha_{k}-1<\alpha_{k} \leq n$ satisfying $\alpha_{k-1} \leq i<\alpha_{k}$ and $\alpha_{t}=\min \left\{\alpha: \alpha>\alpha_{t-1}, \pi_{\alpha_{t-1}-1} \pi_{\alpha} \in E(G)\right\}$ for $t \in[k]$.

We write $P(p, q)$ for the sequence $\pi_{p} \pi_{p+1} \cdots \pi_{q}$ when $p \leq q$ and for the sequence $\pi_{p} \pi_{p-1} \cdots \pi_{q}$ when $p \geq q$. Let $j=\alpha_{k-1}-1$. If $k-1$ is even, say $k-1=2 t$, then the required spanning $\pi_{i}, \pi_{j}$-path can be chosen as $P\left(\alpha_{2 t}-1, \alpha_{2 t-1}\right) P\left(\alpha_{2 t-2}-1, \alpha_{2 t-3}\right) \cdots P\left(\alpha_{2}-1, \alpha_{1}\right) P\left(\alpha_{0}-1, \alpha_{1}-\right.$ 1) $P\left(\alpha_{2}, \alpha_{3}-1\right) \cdots P\left(\alpha_{2 t-2}, \alpha_{2 t-1}-1\right) P\left(\alpha_{2 t}, i\right)$; If $k-1$ is odd, say $k-1=2 t+1$, then the path can be chosen to be $P\left(\alpha_{2 t+1}-1, \alpha_{2 t}\right) P\left(\alpha_{2 t-1}-1, \alpha_{2 t-2}\right) \cdots P\left(\alpha_{1}-1, \alpha_{0}-1\right) P\left(\alpha_{1}, \alpha_{2}-1\right) P\left(\alpha_{3}, \alpha_{4}-\right.$ 1) $\cdots P\left(\alpha_{2 t-1}, \alpha_{2 t}-1\right) P\left(\alpha_{2 t+1}, i\right)$. This finishes the proof.

Proof of Corollary 2; We apply induction on the number $n$ of vertices of $G$. The result is obvious when $n \leq 3$. Now assume that $n \geq 4$ and the assertion holds for smaller $n$.

Let $\pi_{1} \cdots \pi_{n}$ be a 2-thick Hamiltonian vertex ordering of $G$. Take arbitrarily an $i \in[n]$. We intend to show that $G$ has a Hamiltonian path with $\pi_{i}$ as an endpoint. When $i \in\{1, n\},\left(\pi_{1}, \ldots, \pi_{n}\right)$ is a required path. We thus turn to the case of $1<i<n$. Because $\pi$ is 2-thick, Lemma 97 implies that there is $j \in[i-1]$ such that $G\left[\pi_{1}, \ldots, \pi_{i}\right]$ has a spanning $\pi_{i}, \pi_{j}$-path, say $P$, and that $\pi_{j} \pi_{k} \in E(G)$ for some $k>i$. By the induction assumption, $G\left[\pi_{i+1}, \ldots, \pi_{n}\right]$ has a Hamiltonian path $Q$ starting from $\pi_{k}$. In light of $\pi_{j} \pi_{k} \in E(G)$, we can combine $P$ and $Q$ to obtain a Hamiltonian path of $G$ starting at $\pi_{i}$, completing the proof.

Lemma 98 Let $G$ be a graph and let $\mathcal{V} \subseteq V(G)$. Take $\mathcal{E} \subseteq E(G-\mathcal{V})$ such that $G[\mathcal{E}]$ is a linear forest with one of its components being a $u$, v-path $P$. If $V(G-\mathcal{V}) \backslash V(P) \neq \emptyset$ and $\{u, v\} \cup(V(G-\mathcal{V}) \backslash V(P))$ is a clique in $G$, then $G-\mathcal{V}$ has a Hamiltonian cycle containing all edges in $\mathcal{E}$.

Proof: It is apparent that we can extend the path $P$ to arrive at a required Hamiltonian cycle.

Proof of Theorem 5: We prove the claim by an induction on $n=|V(G)|$. For the base step, namely when $n=k+1$, the graph $G$ is the complete graph $K_{k+1}$ and so the result is trivially true. We now assume $n>k+1$ and the result holds for smaller $n$. 
Let $\pi=\pi_{1} \cdots \pi_{n}$ be a $k$-thick Hamiltonian vertex ordering of $G$. Take any $\mathcal{V} \subseteq V(G)$ and $\mathcal{E} \subseteq$ $E(G-\mathcal{V})$ such that $G[\mathcal{E}]$ is a linear forest. We assume that $|\mathcal{E}|+|\mathcal{V}| \leq k-2$ and we intend to show that $G-\mathcal{V}$ contains a Hamiltonian cycle which passes through all the edges in $\mathcal{E}$.

If $\mathcal{E} \cup \mathcal{V}=\emptyset$, the result is implied by Theorem 1 , and also follows from Lemma 97 by setting $i=n-1$. It remains to consider the case of $\mathcal{E} \cup \mathcal{V} \neq \emptyset$.

CASE $1 . \Im(\mathcal{E})=\emptyset$, namely $\mathcal{E}$ is a matching.

Let $\alpha=\min \left\{i: \pi_{i} \in \partial(\mathcal{E}) \cup \mathcal{V}\right\}$ and $\mathcal{W}=\left\{\pi_{i}: \alpha<i \leq n\right\}$. Pick one endpoint from each edge in $\mathcal{E}$ and add them into $\mathcal{V}$ to form a new set $\overline{\mathcal{V}}$. Note that $|\overline{\mathcal{V}}|=|\mathcal{V}|+|\mathcal{E}| \leq k-2$.

CASE 1.1. $\pi_{\alpha} \in \mathcal{V}$.

CASE 1.1.1. $\alpha=1$.

The result follows from the induction assumption on the graph $G[\mathcal{W}]$.

CASE 1.1.2. $\alpha=2$.

It holds $\left|N_{G}\left(\pi_{1}\right)\right| \geq k>k-2 \geq \mid \overline{\mathcal{V}}$. Henceforth, $\pi_{1}$ has two neighbors outside of $\overline{\mathcal{V}}$, say $\pi_{\beta}$ and $\pi_{\gamma}$. Note that $\pi_{\beta} \pi_{\gamma} \in \mathcal{E}$ will never occur due to our choice of $\overline{\mathcal{V}}$.

CASE 1.1.2.1. $n \leq k+3$.

In this case, $G[\mathcal{W}]$ is a complete graph. Taking $P=\left(\pi_{\beta}, \pi_{1}, \pi_{\gamma}\right)$ and applying Lemma 98 gives the result.

CASE 1.1.2.2. $n>k+3$.

In this case, $\pi_{3} \cdots \pi_{n}$ is a $k$-thick Hamiltonian vertex ordering of $G[\mathcal{W}]$. Let $H$ be the graph $G[\mathcal{W}]+$ $\pi_{\beta} \pi_{\gamma}$. The induction assumption on the graph $H$ says that there is a Hamiltonian cycle $C$ of $H-\left(\mathcal{V} \backslash\left\{\pi_{\alpha}\right\}\right)$ which contains $\mathcal{E} \cup\left\{\pi_{\beta} \pi_{\gamma}\right\}$. Replacing the edge $\pi_{\beta} \pi_{\gamma}$ by the path $\left(\pi_{\beta}, \pi_{1}, \pi_{\gamma}\right)$ on $C$, we yield the required Hamiltonian cycle of $G-\mathcal{V}$ which passes through $\mathcal{E}$.

CASE 1.1.3. $\alpha-1>1$.

On account of Lemma 97, there exist $\beta \in[\alpha-2]$ and a spanning $\pi_{\alpha-1}, \pi_{\beta}$-path $Q$ of $G\left[\pi_{1}, \ldots, \pi_{\alpha-1}\right]$ such that $\left\{\gamma: \gamma>\beta+1, \pi_{\beta} \pi_{\gamma} \in E(G)\right\} \subseteq\{\alpha, \ldots, n\}$. Recall that the ordering $\pi$ is $k$-thick. So, we know that either $G\left[\pi_{\beta}, \ldots, \pi_{n}\right]$ is a complete graph or $\mathrm{d}_{G, \pi}(\beta) \geq k$. For the former case, we can appeal to Lemma 98 and obtain the result. Henceforth, we can assume that $\mathrm{d}_{G, \pi}(\beta) \geq k$, which implies that $\pi_{\beta}$ has one neighbor $v \in\left\{\pi_{\alpha}, \ldots, \pi_{n}\right\} \backslash \overline{\mathcal{V}}=\mathcal{W} \backslash \overline{\mathcal{V}}$. Likewise, we further distinguish two subcases, either $G\left[\pi_{\alpha-1}, \ldots, \pi_{n}\right]$ is a complete graph or $\mathrm{d}_{G, \pi}(\alpha-1) \geq k$.

CASE 1.1.3.1. $G\left[\pi_{\alpha-1}, \ldots, \pi_{n}\right]$ is a complete graph.

By considering the $v, \pi_{\alpha-1}$-path $\left(v, \pi_{\beta}\right) \pi_{\beta} Q$, we can use Lemma 98 again to find a desired Hamiltonian cycle of $G-\mathcal{V}$ passing through $\mathcal{E}$.

CASE 1.1.3.2. $\mathrm{d}_{G, \pi}(\alpha-1) \geq k$ and hence $\pi_{\alpha-1}$ has one neighbor $u \in \mathcal{W} \backslash(\overline{\mathcal{V}} \cup\{v\})$.

Let $H=G[\mathcal{W}]+u v$. We utilize the induction assumption on the graph $H$ to find that $H-\left(\mathcal{V} \backslash\left\{\pi_{\alpha}\right\}\right)$ has a Hamiltonian cycle $C$ passing through all edges from $\mathcal{E}$ as well as $u v$. Substituting the edge $u v$ by $\left(u, \pi_{\alpha-1}\right) \underline{\pi_{\alpha-1}} Q \underline{\pi_{\beta}}\left(\pi_{\beta}, v\right)$ in $C$ results in a Hamiltonian cycle for $G-\mathcal{V}$ containing all edges from $\mathcal{E}$.

CASE 1.2. $\pi_{\alpha} \in \partial(\mathcal{E})$. Let us assume that $\pi_{\alpha} \pi_{\alpha^{\prime}} \in \mathcal{E}$ and $\overline{\mathcal{V}}$ is chosen in a way so that $\pi_{\alpha^{\prime}} \in \overline{\mathcal{V}}$. 
CASE 1.2.1. $\quad \alpha=1$.

Since the ordering $\pi$ is $k$-thick, either $G$ is a complete graph or $\pi_{1}$ has a neighbor $v \notin \overline{\mathcal{V}}$. We need only address the latter case.

Consider the graph $H$ with $V(H)=V(G) \backslash\left\{\pi_{1}\right\}$ and $E(H)=E\left(G-\pi_{1}\right) \cup\left\{\pi_{\alpha^{\prime}} v\right\}$. We use the induction assumption on the graph $H$ and find that $H-\mathcal{V}$ has a Hamiltonian cycle $C$ passing through $\left(\mathcal{E} \backslash\left\{\pi_{1} \pi_{\alpha^{\prime}}\right\}\right) \cup\left\{\pi_{\alpha^{\prime}} v\right\}$. Substituting the edge $\pi_{\alpha^{\prime}} v$ by the path $\left(\pi_{\alpha^{\prime}}, \pi_{1}, v\right)$, we obtain from $C$ a required Hamiltonian cycle of $G-\mathcal{V}$ passing through $\mathcal{E}$.

CASE 1.2.2. $\quad \alpha>1$.

In light of Lemma 97, there exist $\beta \in[\alpha-1]$ and a spanning $\pi_{\alpha}, \pi_{\beta}$-path $Q$ of $G\left[\pi_{1}, \ldots, \pi_{\alpha}\right]$ such that $\left\{\gamma: \gamma>\beta+1, \pi_{\beta} \pi_{\gamma} \in E(G)\right\} \subseteq\{\alpha+1, \ldots, n\}$.

Because $\pi$ is a $k$-thick ordering of $G$, we see that $G\left[\pi_{\beta}, \ldots, \pi_{n}\right]$ is either complete or has Hamiltonian thickness at least $k$. The first case is easily dealt with by Lemma 98 and so we focus attention on the second case. In this case, we can choose $\beta^{\prime}>\alpha$ so that $\pi_{\beta} \pi_{\beta^{\prime}} \in E(G)$ and $\pi_{\beta^{\prime}} \notin \overline{\mathcal{V}}$. Note that $G$ has a $\pi_{\alpha^{\prime}}, \pi_{\beta^{\prime}}$-path $R=\left(\pi_{\alpha^{\prime}}, Q_{1}, \ldots, Q_{\alpha}, \pi_{\beta^{\prime}}\right)$, where $\left(\pi_{\alpha}=Q_{1}, \ldots, Q_{\alpha}=\pi_{\beta}\right)=Q$. Define $H$ to be the graph $G[\mathcal{W}]+\pi_{\alpha^{\prime}} \pi_{\beta^{\prime}}$. The graph $H$ is either complete or has Hamiltonian thickness at least $k$. By the induction assumption, $H-\mathcal{V}$ has a Hamiltonian cycle passing through $\left(\mathcal{E} \backslash\left\{\pi_{\alpha} \pi_{\alpha^{\prime}}\right\}\right) \cup\left\{\pi_{\alpha^{\prime}} \pi_{\beta^{\prime}}\right\}$. Replacing the edge $\pi_{\alpha^{\prime}} \pi_{\beta^{\prime}}$ on this cycle by the path $R$ creates a Hamiltonian cycle of $G-\mathcal{V}$ containing all edges from $\mathcal{E}$, as wanted.

CASE 2. $\Im(\mathcal{E}) \neq \emptyset$.

We may assume that $P_{1}, \ldots, P_{t}$ are all components of $G[\mathcal{E}]$ that contain degree 2 vertices. For each $i \in[t]$, let the two endpoints of $P_{i}$ be $a_{i}$ and $b_{i}$ and let $Q_{i}$ denote the set of remaining vertices on $P_{i}$. Let $H$ be the graph with vertex set $V(H)=V(G)$ and edge set $E(H)=E(G) \cup\left\{a_{i} b_{i}: i \in[t]\right\}$. It is clear that $\pi$ is also a $k$-thick Hamiltonian vertex ordering of $H$. Let $\mathcal{V}^{\prime}=\mathcal{V} \cup\left(\cup_{i \in[t]} Q_{i}\right)$ and let $\mathcal{E}^{\prime}=\left\{a_{i} b_{i}: i \in[t]\right\} \cup\left(\mathcal{E} \backslash \cup_{i \in[t]} E\left(P_{i}\right)\right)$.

According to the analysis for Case $1, H-\mathcal{V}^{\prime}$ has a Hamiltonian cycle $C$ containing $\mathcal{E}^{\prime}$. By replacing the edge $a_{i} b_{i}$ by $P_{i}$ for each $i \in[t]$, we derive from $C$ a required Hamiltonian cycle of $G-\mathcal{V}$ containing $\mathcal{E}$. This is what we wanted to show.

Proof of Theorem 7: The result is trivial when $k=1$. We now assume $k \geq 2$ and so, by Theorem 5, $G$ is $(k-2)$-vertex-super-Hamiltonian. Take any nonnegative integers $t_{1}$ and $t_{2}$ such that $t_{1}+t_{2} \leq k-1$. For any $\mathcal{V} \in\left(\begin{array}{c}V(G) \\ t_{1}\end{array}\right)$ and $\mathcal{E} \in\left(\begin{array}{c}E(G-\mathcal{V}) \\ t_{2}\end{array}\right)$ such that $\mathcal{E}$ is the edge set of a linear forest $F$, we need to find a Hamiltonian path of $G-\mathcal{V}$ passing through $\mathcal{E}$. If $t_{1} \geq 1$, we let $\mathcal{V}^{\prime}=\mathcal{V} \backslash\{v\}$ for one element $v \in \mathcal{V}$ and then, as $G$ is $(k-2)$-vertex-super-Hamiltonian, we can find a Hamiltonian cycle $C$ of $G-\mathcal{V}^{\prime}$ passing through $\mathcal{E}$, and then clearly the path obtained from $C$ by removing the vertex $v$ is a required Hamiltonian path of $G-\mathcal{V}$. We next consider $t_{1}=0$. If $t_{2} \leq k-2$, we still get the result directly from the fact that $G$ is $(k-2)$-vertex-super-Hamiltonian. We can thus suppose $t_{2}=k-1 \geq 1$. Pick $a b \in \mathcal{E}$ such that $a$ is a degree 1 vertex in the linear forest $F$. Since $G$ is $(k-2)$-vertex-super-Hamiltonian, there exist a Hamiltonian cycle $C$ of $G-\mathcal{V}=G$ passing through $\mathcal{E} \backslash\{a b\}$, say $\left\langle v_{0}, \ldots, v_{m}\right\rangle$, where $v_{0}=b$ and $v_{i}=a$ for $i \in[m]$. If $i=1$, the required Hamiltonian path can be chosen as $\left(v_{1}, v_{0}, v_{m}, v_{m-1}, \ldots, v_{2}\right)$; If $i=m$, we will instead find the Hamiltonian path $\left(v_{m}, v_{0}, v_{1}, v_{2}, \ldots, v_{m-1}\right)$. Now assume that $i \in\{2, \ldots, m-1\}$. As $F$ is a linear forest and $v_{0} v_{i} \in \mathcal{E}$, it is impossible that both $v_{0} v_{1}$ and $v_{0} v_{m}$ fall into $\mathcal{E}$. By symmetry, we 
assume that $v_{0} v_{1} \notin \mathcal{E}$. It is not hard to check that we can set the required Hamiltonian path of $G-\mathcal{V}=G$ that passes through $\mathcal{E}$ to be $\left(v_{1}, \ldots, v_{i}=a, b=v_{0}, v_{m}, v_{m-1}, \ldots, v_{i+1}\right)$, finishing the proof.

Proof of Theorem 9; We induct on $n=|V(G)|$. If $n=k+1$, we have $G=K_{k+1}$ and so the result is straightforward. We now assume $n>k+1$ and the result holds for smaller $n$. We may assume that $G$ is not a complete graph.

Choose $\pi_{1} \cdots \pi_{n}$ to be a $k$-thick Hamiltonian vertex ordering of $G$. Fix $\mathcal{V} \subseteq V(G)$ and $\mathcal{E} \subseteq E(G-\mathcal{V})$ such that the edge induced graph $G[\mathcal{E}]$ is a linear forest. Take integers $s$ and $t$ such that $1 \leq s<t \leq n$ and $\pi_{s}$ and $\pi_{t}$ fall into $V(G) \backslash(\mathcal{V} \cup \Im(\mathcal{E}))$ and do not lie in a common component of $G[\mathcal{E}]$. We assume that $|\mathcal{E}|+|\mathcal{V}| \leq k-3$ and we aim to show that $G-\mathcal{V}$ contains a spanning $\pi_{s}, \pi_{t}$-path passing through all the edges in $\mathcal{E}$.

Let $\alpha=\min \left\{i: \pi_{i} \in\left\{\pi_{s}\right\} \cup \mathcal{V} \cup \partial(\mathcal{E})\right\}, \mathcal{W}=\left\{\pi_{i}: \alpha<i \leq n\right\}$ and $H=G[\mathcal{W}]$.

CASE $1 . \Im(\mathcal{E})=\emptyset$, namely $\mathcal{E}$, which may be empty, consists of a set of independent edges.

Pick one endpoint from each edge in $\mathcal{E}$ and add them into $\mathcal{V}$ to form a new set $\overline{\mathcal{V}} \in\left(\begin{array}{c}V(G) \\ \leq k-3\end{array}\right)$. In many cases, we can arbitrarily choose one point between the two endpoints of an edge in $\mathcal{E}$ as a member of $\overline{\mathcal{V}}$. But in some cases, we need to be more careful with our choice in order to make the induction proof work. That is, in different subcases we encounter below, we may construct different $\overline{\mathcal{V}}$.

CASE 1.1. $\alpha=s$.

CASE 1.1.1. $\pi_{s} \notin \partial(\mathcal{E})$.

According to Lemma 97, there exist $r \in[s]$ such that $[s] \cap\left\{q: q>r+1, \pi_{q} \pi_{r} \in E(G)\right\}=\emptyset$ and a Hamiltonian path $Q$ of $G\left[\pi_{1}, \ldots, \pi_{s}\right]$ leading from $\pi_{s}$ to $\pi_{r}$. It remains to find a neighbor $\pi_{s^{\prime}}$ of $\pi_{r}$ in $\mathcal{W}$ as well as a spanning $\pi_{s^{\prime}}, \pi_{t}$-path of $H-\mathcal{V}$ passing through all the edges in $\mathcal{E}$.

If $G\left[\pi_{r}, \ldots, \pi_{n}\right]$ is a complete graph, the existence of such a path is self-evident. Otherwise, $\pi_{r}$ has at least $k-1$ neighbors inside $\mathcal{W}$ and so we can choose one of its neighbors from them, say $\pi_{s^{\prime}}$, such that $\pi_{s^{\prime}} \notin \overline{\mathcal{V}} \cup\left\{\pi_{t}\right\}$. Let us assume that $\pi_{t} \notin \overline{\mathcal{V}}$. Noting $\Im(\mathcal{E})=\emptyset$, we see that $\pi_{s^{\prime}}$ and $\pi_{t}$ do not appear in the same component of $G[\mathcal{E}]$. Either $H$ is a complete graph or we are allowed to apply the induction assumption on $H$, guaranteeing the existence of the wanted $\pi_{s^{\prime}}, \pi_{t}$-path of $H-\mathcal{V}$.

CASE 1.1.2. $\pi_{s} \in \partial(\mathcal{E})$.

Let $s^{\prime}$ be the unique number such that $\pi_{s} \pi_{s^{\prime}} \in \mathcal{E}$.

CASE 1.1.2.1. $s=1$.

Clearly, our task is now to find a spanning $\pi_{s^{\prime}}, \pi_{t}$-path of $H-\mathcal{V}$ which passes through all edges in $\mathcal{E} \backslash\left\{\pi_{1} \pi_{s^{\prime}}\right\}$ and this simply follows from the induction assumption.

CASE 1.1.2.2. $s \geq 2$.

By Lemma 97 there exist $r \in[s-1]$ such that $[s-1] \cap\left\{q: q>r+1, \pi_{q} \pi_{r} \in E(G)\right\}=\emptyset$ and a Hamiltonian path $Q$ of $G\left[\pi_{1}, \ldots, \pi_{s-1}\right]$ leading from $\pi_{s-1}$ to $\pi_{r}$.

If $G\left[\pi_{s-1}, \ldots, \pi_{n}\right]$ is a complete graph, by distinguishing whether or not $G\left[\pi_{r}, \ldots, \pi_{n}\right]$ is a complete graph, it is routine to confirm the existence of a spanning $\pi_{s}, \pi_{t}$-path of $G-\mathcal{V}$ which passes through all the edges in $\mathcal{E}$.

Suppose for the moment that $G\left[\pi_{s-1}, \ldots, \pi_{n}\right]$ is not a complete graph. Since $\pi$ is $k$-thick, both $\pi_{s-1}$ and $\pi_{r}$ have at least $k-1$ neighbors in $\mathcal{W}$. Considering that $|\overline{\mathcal{V}}| \leq k-3$, we can pick $\{u, w\} \in\left(\begin{array}{c}\mathcal{W} \backslash \overline{\mathcal{V}} \\ 2\end{array}\right)$ 
so that $u \pi_{r}, w \pi_{s-1} \in E(G)$. Note that $H$ is either a complete graph or has a $k$-thick Hamiltonian vertex ordering. We choose the set $\overline{\mathcal{V}}$ in a way to guarantee $\left\{\pi_{s}, \pi_{t}\right\} \cap \overline{\mathcal{V}}=\emptyset$, which then tells us that $\left(\mathcal{E} \backslash\left\{\pi_{s} \pi_{s^{\prime}}\right\}\right) \cup\{u w\}$ induces a linear forest in $H$ of which no component contains both $\pi_{t}$ and $\pi_{s^{\prime}}$. So, the induction assumption ensures that we can find a spanning $\pi_{s^{\prime}}, \pi_{t}$-path of $H-\mathcal{V}$ which traverses through all edges in $\left(\mathcal{E} \backslash\left\{\pi_{s} \pi_{s^{\prime}}\right\}\right) \cup\{u w\}$. Replacing $u w$ in this path with the $u, w$-path $\left(u, \pi_{r}\right) \underline{\pi_{r}} Q \underline{\pi_{s-1}}\left(\pi_{s-1}, w\right)$ and then adjoining it to $\pi_{s} \pi_{s^{\prime}}$ at $\pi_{s^{\prime}}$ gives the desired Hamiltonian path of $G-\mathcal{V}$.

CASE 1.2. $\pi_{\alpha} \in \mathcal{V}$.

In this case, we require that $\overline{\mathcal{V}}$ is chosen so that the following hold: If $\pi_{s} \in \partial(\mathcal{E})$, then $\pi_{s} \notin \overline{\mathcal{V}}$; If $\pi_{t} \in \partial(\mathcal{E})$, then $\pi_{t} \in \overline{\mathcal{V}}$.

CASE 1.2.1. $\alpha=1$.

The result is trivially true since $\pi_{2} \cdots \pi_{n}$ is a $k$-thick Hamiltonian vertex ordering of $G-\pi_{1}$.

CASE 1.2.2. $\alpha \geq 2$.

By Lemma 97, we can find a number $\beta \in[\alpha-1]$ such that $[\alpha-1] \cap\left\{q: q>\beta+1, \pi_{q} \pi_{\beta} \in E(G)\right\}=\emptyset$ as well as a spanning $\pi_{\alpha-1}, \pi_{\beta}$-path $Q$ of $G\left[\pi_{1}, \ldots, \pi_{\alpha-1}\right]$.

By further dividing into two subcases, depending on whether or not $G\left[\pi_{\beta}, \ldots, \pi_{n}\right]$ is a complete graph, the case that $G\left[\pi_{\alpha-1}, \ldots, \pi_{n}\right]$ is a complete graph can be easily disposed of.

We now turn to the case that $G\left[\pi_{\alpha-1}, \ldots, \pi_{n}\right]$ is not a complete graph. Recall that $\pi$ is a $k$-thick Hamiltonian vertex ordering of $G$ while $|\mathcal{W} \cap \overline{\mathcal{V}}|=\left|\overline{\mathcal{V}} \backslash\left\{\pi_{\alpha}\right\}\right| \leq k-4$. Accordingly, we can choose $\{u, w\} \in\left(\begin{array}{c}\mathcal{W} \backslash\left(\overline{\mathcal{V}} \cup\left\{\pi_{s}\right\}\right) \\ 2\end{array}\right)$ so that $u \pi_{\beta}, w \pi_{\alpha-1} \in E(G)$.

It is not hard to check that $u w \notin \mathcal{E}, H[\mathcal{E} \cup\{u w\}]$ is a linear forest, and more importantly, due to the requirement on $\overline{\mathcal{V}}$, the two vertices $\pi_{s}$ and $\pi_{t}$ from $V(H) \backslash(\mathcal{V} \cup \Im(\mathcal{E} \cup\{u w\}))$ cannot fall into the same component of $H[\mathcal{E} \cup\{u w\}]$. We can now conclude from our induction assumption that there exists a spanning $\pi_{s}, \pi_{t}$-path in $(H+u w)-\left(\mathcal{V} \backslash\left\{\pi_{\alpha}\right\}\right)$ passing through $\mathcal{E} \cup\{u w\}$. Replacing the edge $u w$ by $\left(u, \pi_{\beta}\right) \pi_{\beta} Q \pi_{\alpha-1}\left(\pi_{\alpha-1}, w\right)$ then gives rise to the required spanning $\pi_{s}, \pi_{t}$-path in $G-\mathcal{V}$.

CASE 1.3. $\alpha \neq s$ and $\pi_{\alpha} \in \partial(\mathcal{E})$. We suppose that $\alpha^{\prime}$ is the index such that $\pi_{\alpha} \pi_{\alpha^{\prime}} \in \mathcal{E}$.

It follows from Lemma 97 that there is $\beta \in[\alpha]$ such that $G\left[\pi_{1}, \ldots, \pi_{\alpha}\right]$ has a spanning $\pi_{\alpha}, \pi_{\beta}$-path $Q$ and that either $\pi_{\beta}$ has at least $k-1$ neighbors in $\mathcal{W}$ or $G\left[\pi_{\beta}, \ldots, \pi_{n}\right]$ is a complete graph. There is nothing to prove when $G\left[\pi_{\beta}, \ldots, \pi_{n}\right]$ is a complete graph and so we assume that $\pi_{\beta}$ has at least $k-1$ neighbors in $\mathcal{W}$.

CASE 1.3.1. $\alpha^{\prime}=s$.

We require $\pi_{s} \in \overline{\mathcal{V}}$ and $\pi_{t} \notin \overline{\mathcal{V}}$. Choose a neighbor $v$ of $\pi_{\beta}$ in $\mathcal{W} \backslash\left(\overline{\mathcal{V}} \cup\left\{\pi_{t}\right\}\right)$. Let $H^{\prime}=H+\pi_{s} v$. The induction assumption now dictates that $H^{\prime}-\mathcal{V}$ has a spanning $\pi_{s}, \pi_{t}$-path $R$ passing through $(\mathcal{E} \backslash$ $\left.\left\{\pi_{\alpha} \pi_{s}\right\}\right) \cup \pi_{s} v$. Replacing the edge $\pi_{s} v$ by the $\pi_{s}, v$-path $\left(\pi_{s}, \pi_{\alpha}\right) \underline{\pi_{\alpha}} Q \underline{\pi_{\beta}}\left(\pi_{\beta}, v\right)$, we turn the path $R$ into a required spanning $\pi_{s}, \pi_{t}$-path of $G-\mathcal{V}$ covering all edges in $\mathcal{E}$.

CASE 1.3.2. $\quad \alpha^{\prime}=t$.

Swapping the role of $\pi_{s}$ and $\pi_{t}$, we can proceed as in Case 1.3.1 and draw the required conclusion.

CASE 1.3.3. $\alpha^{\prime} \notin\{s, t\}$.

When choosing the set $\mathcal{V}$, we ensure that $\left\{\pi_{s}, \pi_{t}\right\} \cap(\partial \mathcal{E} \backslash \overline{\mathcal{V}})=\emptyset$. Take a neighbor $v$ of $\pi_{\beta}$ in $\mathcal{W} \backslash \overline{\mathcal{V}}$. Let $H^{\prime}$ stand for $H+\pi_{\alpha^{\prime}} v$. An application of the induction assumption on $H^{\prime}$ yields a spanning $\pi_{s}, \pi_{t}$-path $R$ of $H^{\prime}-\mathcal{V}$ passing through all edges of $\left(\mathcal{E} \backslash\left\{\pi_{\alpha} \pi_{\alpha^{\prime}}\right\}\right) \cup\left\{\pi_{\alpha^{\prime}} v\right\}$. To go from this path $R$ to a required 
spanning $\pi_{s}, \pi_{t}$-path of $G-\mathcal{V}$ containing all edges of $\mathcal{E}$, what is to be done is just to replace the edge $\pi_{\alpha^{\prime}} v$ by the path $\left(\pi_{\alpha^{\prime}}, \pi_{\alpha}\right) \underline{\pi_{\alpha}} Q \pi_{\beta}\left(\pi_{\beta}, v\right)$, finishing the proof.

CASE 2. $\Im(\mathcal{E}) \neq \emptyset$.

Analogous to the proof of Theorem 5 , this case follows from Case 1.

\subsubsection{Proofs of Theorems 10,12 and 13}

Proof of Theorem 10; Let $n=|V(G)|$ and let $\pi=\pi_{1} \cdots \pi_{n}$ be a $(2 k+2)$-thick Hamiltonian vertex ordering of $G$. Fix a subgraph $F$ of $G$ which is a linear forest of size not greater than $k$. We enumerate the components of $F$ as $\mathscr{O}_{1}, \ldots, \mathscr{O}_{\ell}$. Let $\mathcal{E}=E(F)$ and $\mathcal{V}=V(F) \backslash(\partial(\mathcal{E}) \cup \Im(\mathcal{E}))$. Take arbitrarily $\{s, t\} \in$ $\left(\begin{array}{c}{[n]} \\ 2\end{array}\right)$ such that $\pi_{s} \in(V(G) \backslash V(F)) \cup\left(V\left(\mathscr{O}_{1}\right) \backslash \Im\left(\mathscr{O}_{1}\right)\right)$ and $\pi_{t} \in(V(G) \backslash V(F)) \cup\left(V\left(\mathscr{O}_{\ell}\right) \backslash \Im\left(\mathscr{O}_{\ell}\right)\right)$. We require that $\pi_{s}$ and $\pi_{t}$ do not fall into the same component of $F$. We aim to find a spanning $\pi_{s}, \pi_{t}$-path of $G$ which has $F$ as a subgraph and traverses its components $\mathscr{O}_{1}, \ldots$, and $\mathscr{O}_{\ell}$ in this order. Note that, without loss of generality, we may assume that $\pi_{s}, \pi_{t} \notin \mathcal{V}$. Besides that, we can even assume that $s<t-$ Otherwise, consider $s^{\prime}=t, t^{\prime}=s, \mathscr{O}_{i}^{\prime}=\mathscr{O}_{\ell+1-i}$ for $i \in[\ell]$ and continue the proof for the new parameters $s^{\prime}, t^{\prime}$, and $\mathscr{O}_{i}^{\prime}$ for $i \in[\ell]$.

We carry out an induction on $n$. When $n \leq 2 k+3$, the graph $G$ is a complete graph and so the result is trivially true. We now assume that $n>2 k+3$ and the result holds for smaller $n$.

If $|\mathcal{E}|+|\mathcal{V}| \leq 1$, the result is straightforward from Theorem 9 We hereafter assume that $2 \leq|\mathcal{E}|+|\mathcal{V}| \leq$ $k$. Let $\alpha=\min \left\{i: \pi_{i} \in V(F)\right\}$, let $\mathcal{W}=\left\{\pi_{i}: \alpha+1 \leq i \leq n\right\}$ and let $j \in[\ell]$ be the index such that $\pi_{\alpha} \in V\left(\mathscr{O}_{j}\right)$.

CASE $1 . \quad \alpha<s$.

CASE 1.1. $\pi_{\alpha} \in \mathcal{V}$.

In this case,

$$
\begin{aligned}
|V(F)| & =\left|\left\{\pi_{\alpha}\right\}\right|+\left|\mathcal{V} \backslash\left\{\pi_{\alpha}\right\}\right|+2|\mathcal{E}|-|\Im(\mathcal{E})| \\
& \leq 1+2(|\mathcal{V}|-1)+2|\mathcal{E}| \\
& =1+2(|\mathcal{V}|+|\mathcal{E}|-1) \\
& \leq 2 k-1
\end{aligned}
$$

CASE 1.1.1. $\quad \alpha=1$.

Since $\pi$ is $(2 k+2)$-thick, $\pi_{1}$ has two neighbors, say $\pi_{\beta}$ and $\pi_{\gamma}$, which are outside of $V(F) \cup\left\{\pi_{s}, \pi_{t}\right\}$. Let $H$ denote the graph $G[\mathcal{W}]+\pi_{\beta} \pi_{\gamma}$. Let $F^{\prime}$ be the ordered linear forest obtained from the ordered linear forest $(F, \mathscr{O})$ by replacing $\mathscr{O}_{j}$ with $\left(\pi_{\beta}, \pi_{\gamma}\right)$.

Applying the induction assumption on $H$ yields a spanning $\pi_{s}, \pi_{t}$-path $P$ of $H$ passing through the ordered linear forest $F^{\prime}$. Replacing the edge $\pi_{\beta} \pi_{\gamma}$ by the path $\left(\pi_{\beta}, \pi_{1}, \pi_{\gamma}\right)$ on $P$, we yield the required spanning $\pi_{s}, \pi_{t}$-path of $G$ which traverses the ordered linear forest $F$, as required.

CASE 1.1.2. $\quad \alpha>1$

On account of Lemma 97, we can take a $\beta \in[\alpha-1]$ and a spanning $\pi_{\alpha}, \pi_{\beta}$-path $Q$ of $G\left[\pi_{1}, \ldots, \pi_{\alpha}\right]$ such that $\left\{\gamma: \gamma>\beta+1, \pi_{\beta} \pi_{\gamma} \in E(G)\right\} \subseteq\{\alpha+1, \ldots, n\}$. Recall that the ordering $\pi$ is $(2 k+2)$ thick. So, we know from Eq. (14) that either $G\left[\pi_{\beta}, \ldots, \pi_{n}\right]$ is a complete graph or $\pi_{\beta}$ has one neighbor $v \in \mathcal{W} \backslash\left\{V(F) \cup\left\{\pi_{s}, \pi_{t}\right\}\right\}$. 
The former case is quite trivial. For the latter case, letting $F^{\prime}$ be the ordered linear forest obtained from $(F, \mathscr{O})$ by replacing $\mathscr{O}_{j}$ with a new component $\left(\pi_{\alpha}, v\right)$, an application of the induction assumption on the graph $H=G\left[\pi_{\alpha}, \ldots, \pi_{n}\right]+\pi_{\alpha} v$ shows that $H$ has a spanning $\pi_{s}, \pi_{t}$-path $P$ passing through $F^{\prime}$. Replacing the edge $\pi_{\alpha} v$ by $Q \pi_{\beta}\left(\pi_{\beta}, v\right)$ in $P$ results in a spanning $\pi_{s}, \pi_{t}$-path of $G$ passing through the ordered linear forest $F$.

CASE 1.2. $\pi_{\alpha} \in \partial(\mathcal{E})$.

By Lemma 97, there exist $\beta \in[\alpha]$ and a spanning $\pi_{\alpha}, \pi_{\beta}$-path $Q$ of $G-\mathcal{W}$ such that $\left\{\pi_{\gamma}: \gamma>\right.$ $\left.\beta+1, \pi_{\beta} \pi_{\gamma} \in E(G)\right\} \subseteq \mathcal{W}$. Suppose that $\alpha^{\prime} \in[n]$ is the index such that $\pi_{\alpha} \pi_{\alpha^{\prime}} \in \mathcal{E}$.

CASE 1.2.1. $\left(N_{G}\left(\pi_{\beta}\right) \cap \mathcal{W}\right) \backslash\left\{V(F) \cup\left\{\pi_{s}, \pi_{t}\right\}\right\} \neq \emptyset$.

Pick $u \in\left(N_{G}\left(\pi_{\beta}\right) \cap \mathcal{W}\right) \backslash\left\{V(F) \cup\left\{\pi_{s}, \pi_{t}\right\}\right\}$ and let $H=G[\mathcal{W}]+\pi_{\alpha^{\prime}} u$. Note that $H$ is either a complete graph or contains a $(2 k+2)$-thick Hamiltonian vertex ordering $\pi_{\alpha+1} \cdots \pi_{n}$. In the graph $H$ we connect the path $\mathscr{O}_{j}-\pi_{\alpha}$ and the path $\left(\pi_{\alpha^{\prime}}, u\right)$ at their common endpoint $\pi_{\alpha^{\prime}}$ to form a new path which we denote by $\mathscr{O}_{j}^{\prime}$. Consider the ordered linear forest $F^{\prime}$ that is obtained from $(F, \mathscr{O})$ by replacing the component $\mathscr{O}_{j}$ by $\mathscr{O}_{j}^{\prime}$. We can find a spanning $\pi_{s}, \pi_{t}$-path $R$ of $H$ passing through the ordered linear forest $F^{\prime}$, which follows from the induction assumption on $H$ when $H$ is not a complete graph and is quite trivial when $H$ is complete. Substituting the path $\left(\pi_{\alpha^{\prime}}, \pi_{\alpha}\right) \pi_{\alpha} Q \pi_{\beta}\left(\pi_{\beta}, u\right)$ for the edge $\left(\pi_{\alpha^{\prime}}, u\right)$ in $R$, we

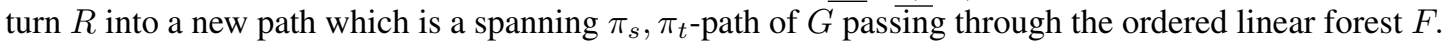

CASE 1.2.2. $\left(N_{G}\left(\pi_{\beta}\right) \cap \mathcal{W}\right) \backslash\left\{V(F) \cup\left\{\pi_{s}, \pi_{t}\right\}\right\}=\emptyset$.

CASE 1.2.2.1. $|\mathcal{W}| \geq 2 k+2$.

Since $|\mathcal{W}| \geq 2 k+2, \pi_{\beta} \cdots \pi_{n}$ is a $(2 k+2)$-thick Hamiltonian vertex ordering of $G\left[\pi_{\beta}, \ldots, \pi_{n}\right]$. Our assumption on $\pi_{\beta}$ then implies that

$$
\left|N_{G}\left(\pi_{\beta}\right) \cap \mathcal{W}\right| \geq \mathrm{d}_{G, \pi}(\beta)-1 \geq 2 k+1
$$

Further observe that

$$
N_{G}\left(\pi_{\beta}\right) \cap \mathcal{W} \subseteq\left(V(F) \backslash\left\{\pi_{\alpha}\right\}\right) \cup\left\{\pi_{s}, \pi_{t}\right\}
$$

and that

$$
|V(F)|=|\mathcal{V}|+2|\mathcal{E}|-|\Im(\mathcal{E})| \leq 2(|\mathcal{V}|+|\mathcal{E}|) \leq 2 k
$$

Putting together Eqs. [15), (16) and (17), we now find that $N_{G}\left(\pi_{\beta}\right) \cap \mathcal{W}=\left(V(F) \backslash\left\{\pi_{\alpha}\right\}\right) \cup\left\{\pi_{s}, \pi_{t}\right\}$, $|V(F)|=2 k,|\Im(\mathcal{E})|=|\mathcal{V}|=0, k=\ell$ and each $\mathscr{O}_{i}, i \in[\ell]$, is a length 1 path, say $\left(a_{i}, b_{i}\right)$, and $\left\{\pi_{s}, \pi_{t}\right\} \cap V(F)=\emptyset$. We assume that $\pi_{\alpha}=a_{j}$ and $\pi_{\alpha^{\prime}}=b_{j}$ for an index $j \in[\ell]=[k]$.

If $j<\ell$, let $u=a_{j+1} \in N_{G}\left(\pi_{\beta}\right)$, let $H=G[\mathcal{W}]+b_{j} u$ and let $F^{\prime}$ be the linear forest whose components are $\mathscr{O}_{1}, \ldots, \mathscr{O}_{j-1}, \mathscr{O}_{j}^{\prime}, \mathscr{O}_{j+2}, \ldots, \mathscr{O}_{\ell}$ in this order where $\mathscr{O}_{j}^{\prime}=\left(b_{j}, u, b_{j+1}\right)$; If $j=\ell$, let $u=\pi_{t} \in N_{G}\left(\pi_{\beta}\right)$, let $H=G[\mathcal{W}]+b_{j} u$ and let $F^{\prime}$ be the linear forest whose components are $\mathscr{O}_{1}, \ldots, \mathscr{O}_{\ell-1}, \mathscr{O}_{\ell}^{\prime}$ in this order where $\mathscr{O}_{\ell}^{\prime}=\left(b_{j}, u\right)$. By the induction hypothesis, $H$ has a spanning $\pi_{s}, \pi_{t}$-path $R$ which traverses the ordered linear forest $F^{\prime}$. Replacing the edge $b_{j} u$ in the path $R$ by the subpath $\left(b_{j}, a_{j}\right) a_{j} Q \pi_{\beta}\left(\pi_{\beta}, u\right)$ yields a new path which is a spanning $\pi_{s}, \pi_{t}$-path of $G$ passing through the ordered linear forest $\bar{F}$, as desired. 
CASE 1.2.2.2. $|\mathcal{W}| \leq 2 k+1$.

If $|\mathcal{W}| \leq 2 k+1$, taking into account of the fact that $\pi$ is $(2 k+2)$-thick, we see that $\mathcal{W}$ is a clique of $G$ and $N_{G}\left(\pi_{\beta}\right) \cap \mathcal{W}=\left(\left\{V(F) \cup\left\{\pi_{s}, \pi_{t}\right\}\right\}\right) \backslash\left\{\pi_{\alpha}\right\}$. Let

$$
\mathscr{O}_{i}^{\prime}=\left\{\begin{aligned}
\mathscr{O}_{i}, & \text { if } i \in[\ell] \backslash\{j\}, \\
\mathscr{O}_{i}-\pi_{\alpha}, & \text { if } i=j,
\end{aligned}\right.
$$

and let $F^{\prime}$ be the ordered linear forest of $G[\mathcal{W}]$ whose ordered components are given by $\mathscr{O}^{\prime}$. It is clear that $G[\mathcal{W}]$ has a spanning $\pi_{s}, \pi_{t}$-path $R$ passing the ordered linear forest $F^{\prime}$. Let $v$ be a vertex of $\mathcal{W} \backslash \mathscr{O}_{j}^{\prime}$ which is adjacent to $\pi_{\alpha^{\prime}}$ on the path $R$. We now replace the edge $\pi_{\alpha^{\prime}} v$ by the path $\left(\pi_{\alpha^{\prime}}, \pi_{\alpha}\right) \pi_{\alpha} Q \pi_{\beta}\left(\pi_{\beta}, v\right)$ and then obtain from $R$ a spanning $\pi_{s}, \pi_{t}$-path of $G$ passing through the ordered linear forest $F$, as wanted.

CASE 1.3. $\pi_{\alpha} \in \Im(\mathcal{E})$.

Let us assume that $\left\{\pi_{\alpha} \pi_{\alpha^{\prime}}, \pi_{\alpha} \pi_{\alpha^{\prime \prime}}\right\} \in\left(\begin{array}{c}\mathcal{E} \\ 2\end{array}\right)$. Noting that $\left|E\left(\mathscr{O}_{j}\right)\right| \geq 2$ and so $\left|E\left(\mathscr{O}_{j}\right)\right| \leq 2\left(\left|E\left(\mathscr{O}_{j}\right)\right|-1\right)$. Also note that $\left|E\left(\mathscr{O}_{i}\right)\right|+1 \leq 2\left|E\left(\mathscr{O}_{i}\right)\right|$ whenever $\left|E\left(\mathscr{O}_{i}\right)\right| \geq 1$. It then follows

$$
\begin{aligned}
|V(F)| & =\sum_{i=1}^{\ell}\left(\left|E\left(\mathscr{O}_{i}\right)\right|+1\right) \\
& \leq|\mathcal{V}|+2 \sum_{i \in[\ell] \backslash\{j\}}\left|E\left(\mathscr{O}_{i}\right)\right|+\left(2\left(\left|E\left(\mathscr{O}_{j}\right)\right|-1\right)+1\right) \\
& \leq 2(|\mathcal{V}|+|\mathcal{E}|)-1 \\
& \leq 2 k-1
\end{aligned}
$$

CASE 1.3.1. $\quad \alpha=1$.

Because the ordering $\pi$ is $(2 k+2)$-thick, either $G$ is a complete graph or $\pi_{2} \cdots \pi_{n}$ is a $(2 k+2)$-thick Hamiltonian vertex ordering of $G-\pi_{1}$. We need only consider the latter case.

We adopt the shorthand $H$ for $\left(G-\pi_{\alpha}\right)+\pi_{\alpha^{\prime}} \pi_{\alpha^{\prime \prime}}$. Note that $O^{\prime}=\left(\mathscr{O}_{j}-\pi_{\alpha}\right)+\pi_{\alpha^{\prime}} \pi_{\alpha^{\prime \prime}}$ is a path in $H$. Let $F^{\prime}$ be the ordered linear forest obtained from $F$ by substituting $O^{\prime}$ for $\mathscr{O}_{j}$.

We use the induction assumption on the graph $H$ and find a spanning $\pi_{s}, \pi_{t}$-path $P$ of $H$ passing through the ordered linear forest $F^{\prime}$. Replacing the edge $\pi_{\alpha^{\prime}} \pi_{\alpha^{\prime \prime}}$ by the path $\left(\pi_{\alpha^{\prime}}, \pi_{1}, \pi_{\alpha^{\prime \prime}}\right)$, we obtain from $P$ a required spanning $\pi_{s}, \pi_{t}$-path of $G$ passing through $F$.

CASE 1.3.2. $\alpha \geq 2$.

Owning to Lemma 97 we can get a $\beta \in[\alpha-1]$ and a spanning $\pi_{\alpha-1}, \pi_{\beta}$-path $Q$ of $G\left[\pi_{1}, \ldots, \pi_{\alpha-1}\right]$ such that $\left\{\gamma: \gamma>\beta+1, \pi_{\beta} \pi_{\gamma} \in E(G)\right\} \subseteq\{\alpha, \ldots, n\}$.

If $|\mathcal{W}| \leq 2 k+1$, we can finish the proof by following the strategy of the proof for Case 1.2.2.2. We thus assume below that $|\mathcal{W}| \geq 2 k+2$ and so $\pi_{\alpha} \cdots \pi_{n}$ is a $(2 k+2)$-thick Hamiltonian vertex ordering of $G\left[\pi_{\alpha}, \ldots, \pi_{n}\right]$. Choose one endpoint of the path $\mathscr{O}_{j}$, say $u$, such that $u \notin\left\{\pi_{s}, \pi_{t}\right\}$. By virtue of Eq. (18), it holds

$$
\left|\left(V(F) \cup\left\{\pi_{s}, \pi_{t}\right\}\right) \cap \mathcal{W}\right|=\left|\left(V(F) \backslash\left\{\pi_{\alpha}\right\}\right) \cup\left\{\pi_{s}, \pi_{t}\right\}\right| \leq 2 k .
$$

Henceforth, we can pick $\pi_{\gamma^{\prime}}$ from $\left(N_{G}\left(\pi_{\beta}\right) \cap \mathcal{W}\right) \backslash\left(\left\{\pi_{s}, \pi_{t}\right\} \cup V(F)\right)$ and then pick $\pi_{\gamma^{\prime \prime}}$ from $\left(N_{G}\left(\pi_{\alpha-1}\right) \cap\right.$ $\mathcal{W}) \backslash\left(\left\{\pi_{s}, \pi_{t}, \pi_{\gamma^{\prime}}\right\} \cup(V(F) \backslash\{u\})\right)$. 
Denote by $H$ the graph $G[\mathcal{W}]+\left\{\pi_{\alpha^{\prime}} \pi_{\alpha^{\prime \prime}}, \pi_{\gamma^{\prime}} \pi_{\gamma^{\prime \prime}}\right\}$. When $\pi_{\gamma^{\prime \prime}}=u$, we let $\mathscr{O}_{j}^{\prime}=\left(\left(\mathscr{O}_{j}-\pi_{\alpha}\right)+\right.$ $\left.\pi_{\alpha^{\prime}} \pi_{\alpha^{\prime \prime}}\right) \underline{u}\left(u, \pi_{\gamma^{\prime}}\right)$ and let $F^{\prime}$ be the ordered linear forest obtained from $F$ by substituting $\mathscr{O}_{j}^{\prime}$ for $\mathscr{O}_{j}$. When $\pi_{\gamma^{\prime \prime}} \neq u$, we let $\mathscr{O}_{j}^{\prime}=\left(\mathscr{O}_{j}-\pi_{\alpha}\right)+\pi_{\alpha^{\prime}} \pi_{\alpha^{\prime \prime}}$, let $O^{\prime}=\left(\pi_{\gamma^{\prime \prime}}, \pi_{\gamma^{\prime}}\right)$, and let $F^{\prime}$ be the ordered linear forest obtained from $F$ by substituting $\mathscr{O}_{j}^{\prime}$ for $\mathscr{O}_{j}$ and inserting a new component $O^{\prime}$ in any place of the component ordering.

We use the induction assumption on the graph $H$ and find a spanning $\pi_{s}, \pi_{t}$-path $P$ of $H$ passing through the ordered linear forest $F^{\prime}$. Replacing the edge $\pi_{\alpha^{\prime}} \pi_{\alpha^{\prime \prime}}$ by the path $\left(\pi_{\alpha^{\prime}}, \pi_{\alpha}, \pi_{\alpha^{\prime \prime}}\right)$ and the edge $\pi_{\gamma^{\prime}} \pi_{\gamma^{\prime \prime}}$ by the path $\left(\pi_{\gamma^{\prime \prime}}, \pi_{\alpha-1}\right) \pi_{\alpha-1} Q \pi_{\beta}\left(\pi_{\beta}, \pi_{\gamma^{\prime}}\right)$, we obtain from $P$ a required spanning $\pi_{s}, \pi_{t}$-path of $G$ traveling across $F$ in the required order.

CASE 2. $\alpha=s$.

First note that it holds $j=1$ in this case.

CASE 2.1. $\pi_{s} \notin \mathcal{V}$.

Let us assume that $\alpha^{\prime}$ is the index satisfying $\pi_{\alpha} \pi_{\alpha^{\prime}} \in \mathcal{E}$.

CASE 2.1.1. $\alpha=s=1$.

Let $F^{\prime}$ be the ordered linear forest obtained from $(F, \mathscr{O})$ by replacing $\mathscr{O}_{j}$ by $\mathscr{O}_{j}-\pi_{\alpha}$. We use the induction assumption on the graph $G[\mathcal{W}]$ and find a spanning $\pi_{\alpha^{\prime}}, \pi_{t}$-path $P$ of $G[\mathcal{W}]$ passing through the ordered linear forest $F^{\prime}$. Let $P^{\prime}=\left(\pi_{\alpha}, \pi_{\alpha^{\prime}}\right) \pi_{\alpha^{\prime}} P$. It is not difficult to check that $P^{\prime}$ is a required spanning $\pi_{s}, \pi_{t}$-path of $G$ passing through the ordered linear forest $F$.

CASE 2.1.2. $\alpha=s>1$.

It follows from Lemma 97 that there exist $\beta \in[\alpha-1]$ and a spanning $\pi_{\alpha-1}, \pi_{\beta}$-path $Q$ of $G\left[\pi_{1}, \ldots, \pi_{\alpha-1}\right]$ such that $\left\{\gamma: \gamma>\beta+1, \pi_{\beta} \pi_{\gamma} \in E(G)\right\} \subseteq\{\alpha, \ldots, n\}$.

If $|\mathcal{W}| \leq 2 k+1$, the claim will easily follow from the same argument as in Case 1.2.2.2. We thus assume that $|\mathcal{W}| \geq 2 k+2$ and so $\pi_{\alpha} \cdots \pi_{n}$ is a $(2 k+2)$-thick Hamiltonian vertex ordering of $G\left[\pi_{\alpha}, \ldots, \pi_{n}\right]$.

We assume that $\mathscr{O}_{j}$ is a $\pi_{\alpha}, u$-path in $G$ for some vertex $u$. Observe that $\left|\left(V(F) \cup\left\{\pi_{s}, \pi_{t}\right\}\right) \cap \mathcal{W}\right|=$ $\left|\left(V(F) \backslash\left\{\pi_{\alpha}\right\}\right) \cup\left\{\pi_{t}\right\}\right| \leq 2 k$. Therefore, we can find $\pi_{\gamma^{\prime}}$ from $\left(N_{G}\left(\pi_{\beta}\right) \cap \mathcal{W}\right) \backslash\left(\left\{\pi_{s}, \pi_{t}\right\} \cup V(F)\right)$ and find $\pi_{\gamma^{\prime \prime}}$ from $\left(N_{G}\left(\pi_{\alpha-1}\right) \cap \mathcal{W}\right) \backslash\left(\left\{\pi_{s}, \pi_{t}, \pi_{\gamma^{\prime}}\right\} \cup(V(F) \backslash\{u\})\right)$. Define $H$ to be $G[\mathcal{W}]+\pi_{\gamma^{\prime}} \pi_{\gamma^{\prime \prime}}$. When $\pi_{\gamma^{\prime \prime}}=u$, we let $\mathscr{O}_{j}^{\prime}=\left(\mathscr{O}_{j}-\pi_{\alpha}\right) \underline{u}\left(u, \pi_{\gamma^{\prime}}\right)$ and let $F^{\prime}$ be the ordered linear forest obtained from $F$ by substituting $\mathscr{O}_{j}^{\prime}$ for $\mathscr{O}_{j}$. Let $O^{\prime}=\left(\pi_{\gamma^{\prime \prime}}, \pi_{\gamma^{\prime}}\right)$. When $\pi_{\gamma^{\prime \prime}} \neq u$ and $\mathscr{O}_{j}=\mathscr{O}_{1}$ is just $\left\{\pi_{\alpha}, u\right\}$, let $F^{\prime}$ be the ordered linear forest obtained from $F$ by replacing $\mathscr{O}_{1}$ with $O^{\prime}$. When $\pi_{\gamma^{\prime \prime}} \neq u$ and $\mathscr{O}_{j}=\mathscr{O}_{1}$ contains vertices other than $\pi_{\alpha}$ and $u$, we let $\mathscr{O}_{j}^{\prime}=\mathscr{O}_{j}-\pi_{\alpha}$, and let $F^{\prime}$ be the ordered linear forest obtained from $F$ by substituting $\mathscr{O}_{j}^{\prime}$ for $\mathscr{O}_{j}$ and inserting a new component $O^{\prime}$ in any place of the component ordering.

We employ the induction hypothesis on the graph $H$ and find a spanning $\pi_{\alpha^{\prime}}, \pi_{t}$-path $P$ of $H$ traversing the ordered linear forest $F^{\prime}$. Replacing the edge $\pi_{\gamma^{\prime}} \pi_{\gamma^{\prime \prime}}$ by the path $\left(\pi_{\gamma^{\prime}}, \pi_{\beta}\right) \pi_{\beta} Q \pi_{\alpha-1}\left(\pi_{\alpha-1}, \pi_{\gamma^{\prime \prime}}\right)$, we obtain from $P \underline{\pi_{\alpha^{\prime}}}\left(\pi_{\alpha^{\prime}}, \pi_{\alpha}\right)$ a required spanning $\pi_{s}, \pi_{t}$-path of $G$ passing through the components of $F$ according to the given ordering $\mathscr{O}$, as wanted.

CASE 2.2. $\pi_{s} \in \mathcal{V}$.

From $2 \leq|\mathcal{E}|+|\mathcal{V}|$ we see that $\ell \geq 2$ and so we can find an $\alpha^{\prime} \in[n] \backslash\{s, t\}$ such that $\pi_{\alpha^{\prime}}$ is an endpoint of the path $\mathscr{O}_{2}$. Let $F^{\prime}=F+\pi_{\alpha} \pi_{\alpha^{\prime}}$ with ordered components $\mathscr{O}_{1}^{\prime}=\mathscr{O}_{1} \pi_{\alpha} \mathscr{O}_{2}$ and $\mathscr{O}_{i+1}^{\prime}=\mathscr{O}_{i+2}$ for $i \in[\ell-2]$. By Case 2.1, $G$ contains a spanning $\pi_{s}, \pi_{t}$-path passing through the components of $F^{\prime}$ according to its component ordering $\mathscr{O}^{\prime}$. It is clear that it is a required Hamiltonian path of $G$ in which the ordered linear forest $(F, \mathscr{O})$ embeds. 
CASE 3. $\alpha>s$.

Again, in view of the argument used in Case 1.2.2.2, we need only consider the case that $n-s \geq$ $2 k+2$. This means that $\pi_{s} \cdots \pi_{n}$ is a $(2 k+2)$-thick Hamiltonian vertex ordering of $G\left[\pi_{s}, \ldots, \pi_{n}\right]$. On account of Lemma 97, there exist $\beta \in[s]$ and a spanning $\pi_{s}, \pi_{\beta}$-path $Q$ of $G\left[\pi_{1}, \ldots, \pi_{s}\right]$ such that $\left\{\gamma: \gamma>\beta+1, \pi_{\beta} \pi_{\gamma} \in E(G)\right\} \subseteq\{s+1, \ldots, n\}$. Let $u$ be one endpoint of the path $\mathscr{O}_{1}$. Then we know that $\pi_{\beta}$ has a neighbor, say $v$, such that $v \in\left(\pi[s+1, n] \backslash\left\{V(F) \cup\left\{\pi_{s}, \pi_{t}\right\}\right\}\right) \cup\{u\}$. We use the induction assumption on the graph $G\left[\pi_{s+1}, \ldots, \pi_{n}\right]$ and find a spanning $v, \pi_{t}$-path $P$ of $G[\mathcal{W}]$ passing through the ordered linear forest $F$. Finally, we can check that $Q \pi_{\beta} v P$ is a required spanning $\pi_{s}, \pi_{t}$-path of $G$ passing through the ordered linear forest $F$.

Prior to the statement of Theorem 35, we have defined the join of two graphs. The next result is an observation on the join of a graph and a complete graph.

Lemma 99 For every graph $G$ and every positive integer $n$, it holds $\mathcal{H}\left(G \bigvee K_{n}\right) \geq \mathcal{H}(G)+n$.

Proof: If $G$ is a complete graph, it is clear that $\mathcal{H}\left(G \bigvee K_{n}\right)=\mathcal{H}(G)+n$. We hereafter assume that $G$ is not complete. Let us take $\pi \in V(G)$ ! such that $\mathcal{H}_{G}(\pi)=\mathcal{H}(G)$ and aim to find $\pi^{\prime} \in V\left(G \bigvee K_{n}\right)$ ! such that $\mathcal{H}_{G \bigvee K_{n}}\left(\pi^{\prime}\right) \geq \mathcal{H}(G)+n$.

If $\mathcal{H}(G) \geq 1$, the required ordering $\pi^{\prime}$ can be chosen to be $\pi \tau$ where $\tau$ is any element from $V\left(K_{n}\right)$ !.

We then turn to the case of $\mathcal{H}(G) \leq 0$. Since $G \neq K_{1}$, along the ordering $\pi$, we will read out of $\pi(G)$ paths (c.f. Eq. (2)), say $P_{1}, \ldots, P_{\pi(G)}$, where $P_{i}=\left(\pi_{t_{i}+1}, \ldots, \pi_{t_{i+1}}\right)$ for $i \in[\pi(G)], t_{1}=0, t_{\pi(G)+1}=$ $|V(G)|$. If $n \leq \pi(G)-1$, construct the vertex ordering $\pi^{\prime}$ of $G \bigvee K_{n}$ from $\pi$ by inserting a vertex from $K_{n}$ inbetween $\pi_{t_{i+1}}$ and $\pi_{t_{i+1}+1}$ for each $i \in[n]$; If $n \geq \pi(G)$, construct the vertex ordering $\pi^{\prime}$ of $G \bigvee K_{n}$ from $\pi$ by inserting a vertex from $K_{n}$ inbetween $\pi_{t_{i+1}}$ and $\pi_{t_{i+1}+1}$ for each $i \in[\pi(G)-1]$ and list the remaining $n+1-\pi(G)$ vertices from $K_{n}$ at the end of the sequence. It is routine to check that $\mathcal{H}_{G \bigvee K_{n}}\left(\pi^{\prime}\right)=\mathcal{H}(G)+n$, implying that $\mathcal{H}\left(G \bigvee K_{n}\right) \geq \mathcal{H}(G)+n$

Proof of Corollary 11: Let $G^{\prime}=G \bigvee K_{2}$. Lemma 99 tells us that $G^{\prime} \in \digamma^{2 k+2}$. By Theorem $10 G^{\prime}$ is $k$-ordered Hamiltonian-connected, from which we can deduce that $G$ is $k$-ordered traceable.

Proof of Theorem 12; Fix a subgraph $F$ of $G$ which is a linear forest of size at most $k$. Given any ordering $\mathscr{O}$ of the components of $F$, we need to show that there is a Hamiltonian cycle of $G$ which is a supergraph of $F$ and encounters the components of $F$ in that specified ordering $\mathscr{O}$.

Let $n=|V(G)|$. We prove the claim by induction on $n$. When $n \leq 2 k+2$, the graph $G$ is a complete graph and so the result trivially holds. We now assume that $n>2 k+2$ and the result holds for smaller $n$.

We put $\mathcal{E}=E(F)$ and $\mathcal{V}=V(F) \backslash(\partial(\mathcal{E}) \cup \Im(\mathcal{E}))$. If $F$ is a linear forest of size zero, namely $\mathcal{E} \cup \mathcal{V}=\emptyset$, the result is straightforward from Theorem 1 It remains to consider the case of $\mathcal{E} \cup \mathcal{V} \neq \emptyset$.

Let $\pi=\pi_{1} \cdots \pi_{n}$ be a $(2 k+1)$-thick Hamiltonian vertex ordering of $G$. Let $\alpha=\min \left\{i: \pi_{i} \in V(F)\right\}$ and $\mathcal{W}=\left\{\pi_{i}: \alpha<i \leq n\right\}$.

CASE 1. $\pi_{\alpha} \in \mathcal{V}$.

We can assume that $\alpha<n$ as otherwise any Hamiltonian cycle, whose existence is guaranteed by Theorem 1. will fulfil the requirement. On account of Lemma 97, there are $\beta \in[\alpha]$ and a spanning $\pi_{\alpha}, \pi_{\beta}$-path $Q$ of $G-\mathcal{W}$ such that $\left\{\gamma: \gamma>\beta+1, \pi_{\beta} \pi_{\gamma} \in E(\bar{G})\right\} \subseteq\{\alpha+1, \ldots, n\}$. Recall that the 
ordering $\pi$ is $(2 k+1)$-thick and note that $\left|V(F) \backslash\left\{\pi_{\alpha}\right\}\right| \leq 2(k-1)=2 k-2$. Accordingly, either $G\left[\pi_{\beta}, \ldots, \pi_{n}\right]$ is a complete graph or $\pi_{\beta}$ has one neighbor $v \in \mathcal{W} \backslash V(F)$.

The former case is quite trivial. To deal with the latter case, we consider the linear forest $F^{\prime}$ that has vertex set $V(F) \cup\{v\}$ and edge set $E(F) \cup\left\{\pi_{\alpha} v\right\}$. We utilize the induction assumption on the graph $H=G\left[\pi_{\alpha}, \ldots, \pi_{n}\right]+\pi_{\alpha} v$ and find that $H$ has a Hamiltonian cycle $C$ passing through the linear forest $F^{\prime}$ in the order inherited from $\mathscr{O}$ by substituting the component $\pi_{\alpha}$ with the component $\left(\pi_{\alpha}, v\right)$. Substituting the edge $\pi_{\alpha} v$ by $Q \pi_{\beta}\left(\pi_{\beta}, v\right)$ in $C$ results in a Hamiltonian cycle of $G$ with the components of $F$ appearing in the given order $\widetilde{\mathscr{O}}$.

CASE 2. $\pi_{\alpha} \in \partial(\mathcal{E})$. Let us assume that $\pi_{\alpha} \pi_{\alpha^{\prime}} \in \mathcal{E}$.

In light of Lemma 97, we can find $\beta \in[\alpha]$ and a spanning $\pi_{\alpha}, \pi_{\beta}$-path $Q$ of $G\left[\pi_{1}, \ldots, \pi_{\alpha}\right]$ such that $\left\{\gamma: \gamma>\beta+1, \pi_{\beta} \pi_{\gamma} \in E(G)\right\} \subseteq\{\alpha+1, \ldots, n\}$. Because $\pi$ is a $(2 k+1)$-thick ordering of $G$, we see that either $G\left[\pi_{\beta}, \ldots, \pi_{n}\right]$ is complete or we can choose $\beta^{\prime}$ so that $\pi_{\beta^{\prime}} \in \mathcal{W} \backslash V(F), \pi_{\beta^{\prime}} \pi_{\beta} \in E(G)$. The first case can be easily disposed of and so we focus attention on the second case. We now have a $\pi_{\alpha^{\prime}}, \pi_{\beta^{\prime}}$-path $R=\left(\pi_{\alpha^{\prime}}, \pi_{\alpha}\right) \underline{\pi_{\alpha}} Q \pi_{\beta}\left(\pi_{\beta}, \pi_{\beta^{\prime}}\right)$ in $G$ on the vertex set $\left\{\pi_{\alpha^{\prime}}, \pi_{\beta^{\prime}}, \pi_{1}, \ldots, \pi_{\alpha}\right\}$. Define $H$ to be $G[\mathcal{W}]+\pi_{\alpha^{\prime}} \pi_{\beta^{\prime}}$. Let $F^{\prime}$ be the linear forest obtained from $F$ by replacing the vertex $\pi_{\alpha}$ with $\pi_{\beta^{\prime}}$ and replacing the edge $\pi_{\alpha} \pi_{\alpha^{\prime}}$ with $\pi_{\beta^{\prime}} \pi_{\alpha^{\prime}}$. Naturally, we have an ordering $\mathscr{O}^{\prime}$ of the components of $F^{\prime}$ following the ordering $\mathscr{O}$.

By the induction assumption on the graph $H$, we can find a Hamiltonian cycle $C$ of $H$ with the components of $F^{\prime}$ appearing in the order $\mathscr{O}^{\prime}$. Substituting the edge $\pi_{\alpha^{\prime}} \pi_{\beta^{\prime}}$ by the path $R$, we obtain from $C$ a required Hamiltonian cycle of $G$ with the components of $F$ appearing in the given order $\mathscr{O}$.

CASE 3. $\pi_{\alpha} \in \Im(\mathcal{E})$. Let us assume that $\pi_{\alpha^{\prime}}$ and $\pi_{\alpha^{\prime \prime}}$ are two different vertices such that

$$
\left\{\pi_{\alpha} \pi_{\alpha^{\prime}}, \pi_{\alpha} \pi_{\alpha^{\prime \prime}}\right\} \subseteq \mathcal{E}
$$

CASE 3.1. $\quad \alpha=1$.

Because the ordering $\pi$ is $(2 k+1)$-thick, either $G$ is a complete graph or $\pi_{2} \cdots \pi_{n}$ is a $(2 k+1)$-thick Hamiltonian vertex ordering of $G-\pi_{1}$. We need only consider the latter case.

Let $H=\left(G-\pi_{1}\right)+\pi_{\alpha^{\prime}} \pi_{\alpha^{\prime \prime}}$ and let $F^{\prime}$ be the linear forest $\left(F-\pi_{1}\right)+\pi_{\alpha^{\prime}} \pi_{\alpha^{\prime \prime}}$. Applying the induction assumption on the graph $H$, we get a Hamiltonian cycle $C$ of $H$ with the components of $F^{\prime}$ appearing in the order coinciding with the order $\mathscr{O}$ of their natural counterparts in $F$. Substituting the edge $\pi_{\alpha^{\prime}} \pi_{\alpha^{\prime \prime}}$ by the path $\left(\pi_{\alpha^{\prime}}, \pi_{1}, \pi_{\alpha^{\prime \prime}}\right)$, we obtain from $C$ a required Hamiltonian cycle of $G$ in which the components of $F$ appear in the given order.

CASE 3.2. $\alpha \geq 2$.

In view of Lemma 97 there exist a $\beta \in[\alpha-1]$ and a spanning $\pi_{\alpha-1}, \pi_{\beta}$-path $Q$ of $G\left[\pi_{1}, \ldots, \pi_{\alpha-1}\right]$ such that $\left\{\gamma: \gamma>\beta+1, \pi_{\beta} \pi_{\gamma} \in E(G)\right\} \subseteq\{\alpha, \ldots, n\}$.

If $G\left[\pi_{\alpha-1}, \ldots, \pi_{n}\right]$ is a complete graph, we can complete the proof by considering two subcases, either $G\left[\pi_{\beta}, \ldots, \pi_{n}\right]$ is a complete graph or not. Consequently, it suffices for our purpose now to assume that $\pi_{\alpha-1} \cdots \pi_{n}$ is a $(2 k+1)$-thick Hamiltonian vertex ordering of $G\left[\pi_{\alpha-1}, \ldots, \pi_{n}\right]$. To proceed, we note that

$$
|V(F)|=|\mathcal{V}|+2|\mathcal{E}|-|\Im(\mathcal{E})| \leq 2(|\mathcal{V}|+|\mathcal{E}|)-|\Im(\mathcal{E})| \leq 2 k-1
$$

Henceforth,

$$
|\mathcal{W} \cap V(F)| \leq\left|V(F) \backslash\left\{\pi_{\alpha}\right\}\right| \leq 2 k-2
$$


This enables us to find two distinct vertices $\pi_{\gamma^{\prime}}$ and $\pi_{\gamma^{\prime \prime}}$ such that $\pi_{\gamma^{\prime}} \in\left(N_{G}\left(\pi_{\beta}\right) \cap \mathcal{W}\right) \backslash V(F)$ and $\pi_{\gamma^{\prime \prime}} \in\left(N_{G}\left(\pi_{\alpha-1}\right) \cap \mathcal{W}\right) \backslash V(F)$. Taking the union of $\left(F-\pi_{\alpha}\right)+\pi_{\alpha^{\prime}} \pi_{\alpha^{\prime \prime}}$ and the one edge path $\left(\pi_{\gamma^{\prime}}, \pi_{\gamma^{\prime \prime}}\right)$ yields a subgraph $F^{\prime}$ of $G$ which is a linear forest of the same size with $F$. Set $H$ to be $G[\mathcal{W}]+\left\{\pi_{\alpha^{\prime}} \pi_{\alpha^{\prime \prime}}, \pi_{\gamma^{\prime}} \pi_{\gamma^{\prime \prime}}\right\}$. By the induction assumption on the graph $H$, we find a Hamiltonian cycle $C$ of $H$ which contains $F^{\prime}$ as a subgraph and passes through the components of $F^{\prime}$ other than $\left(\pi_{\gamma^{\prime}}, \pi_{\gamma^{\prime \prime}}\right)$ in the order corresponding to the ordering of the corresponding components of $F$. Substituting the edge $\pi_{\alpha^{\prime}} \pi_{\alpha^{\prime \prime}}$ by the path $\left(\pi_{\alpha^{\prime}}, \pi_{\alpha}, \pi_{\alpha^{\prime \prime}}\right)$ and the edge $\pi_{\gamma^{\prime}} \pi_{\gamma^{\prime \prime}}$ by the path $\left(\pi_{\gamma^{\prime}}, \pi_{\beta}\right) \pi_{\beta} Q \pi_{\alpha-1}\left(\pi_{\alpha-1} \pi_{\gamma^{\prime \prime}}\right)$, we obtain from $C$ a required Hamiltonian cycle of $G$ with the components of $F$ appearing in the given order.

An $s, s$-pseudo-path in a graph $G$ is either a cycle in $G$ passing through $s$ of length at least 3 , or an edge of $G$ incident with $s$ or a path of length zero consisting of $s$ itself. For $s \neq t$, an $s, t$-pseudo-path in a graph $G$ is any $s, t$-path in $G$. The inner vertices of an $s, t$-pseudo-path is the set of vertices on this $s, t$-pseudo-path other than $s$ and $t$. A graph $G$ is almost rooted $k$-connected if the following holds:

(*) for any set $R=\left\{\left(s_{i}, t_{i}\right) \in V(G) \times V(G): i \in[k]\right\}$, we can find a pseudo-path system of $G$ rooted at $R$, namely a set of $s_{i}, t_{i}$-pseudo-paths $P_{i}$ in $G$ for $i \in[k]$ such that the inner vertices of these $P_{i}$ form a partition of $V(G) \backslash S$, where $S=\cup_{i \in[k]}\left\{s_{i}, t_{i}\right\}$.

Proof of Theorem 13; We will indeed prove a stronger claim.

CLAIM: Let $k$ be a positive integer and let $G$ be either a complete graph or a graph from $\digamma^{2 k+1}$. Then $G$ is almost rooted $k$-connected; That is, $\left(^{*}\right)$ holds.

We make an induction on $n=|V(G)|$. If $G$ is a complete graph, the claim is trivial. If $n \leq 2 k+2$, then $G$ must be a complete graph. Accordingly, we now assume that $n>2 k+2$.

Let $\pi=\pi_{1} \cdots \pi_{n}$ be a $(2 k+1)$-thick Hamiltonian vertex ordering of $G$. Let $S=\cup_{i \in[k]}\left\{s_{i}, t_{i}\right\}$, $\alpha=\min \left\{i: \pi_{i} \in S\right\}$ and $q=\left|\left\{i \in[k]: \pi_{\alpha} \in\left\{s_{i}, t_{i}\right\}\right\}\right|$. Without loss of generality, we assume that $s_{i}=\pi_{\alpha}$ for all $i \in[q]$ and $\pi_{\alpha} \notin \cup_{i \in[k] \backslash[q]}\left\{s_{i}, t_{i}\right\}$.

CASE $1 . \quad \alpha=n$.

Since $2 k+1>2$, Theorem 5 implies that $G$ is a Hamiltonian graph. Consequently, we can take $P_{1}$ to be a Hamiltonian cycle of $G$ and put $P_{1+i}, i \in[k-1]$, to be the length-zero path at vertex $\pi_{n}$, getting the required pseudo-path system.

CASE 2. $\alpha<n$.

By virtue of Lemma 97, there exist $\beta \in[\alpha]$ and a spanning $\pi_{\alpha}, \pi_{\beta}$-path $Q=\left(Q_{1}=\pi_{\alpha}, \ldots, Q_{\alpha}=\pi_{\beta}\right)$ of $G\left[\pi_{1}, \ldots, \pi_{\alpha}\right]$ such that $\left\{\gamma: \gamma>\beta+1, \pi_{\beta} \pi_{\gamma} \in E(G)\right\} \subseteq\{\alpha+1, \ldots, n\}$.

CASE 2.1. $s_{i}=t_{i}=\pi_{\alpha}$ for all $i \in[q]$.

For every $i \in[q-1]$, we set $P_{i}$ to be the length zero path consisting of the only vertex $\pi_{\alpha}$.

When $\alpha=1$, let $P_{q}$ be the length zero path consisting of one vertex $\pi_{1}$ and then apply the induction assumption on $G-\pi_{1}$ will conclude the proof.

When $\alpha>1$, we know that $\beta<\alpha$. If $\pi_{\beta} \pi_{\alpha} \in E(G)$, then $Q$ together with $\pi_{\beta} \pi_{\alpha}$ results in a cycle, which we use as $P_{q}$. The remaining pseudo-paths can now be obtained by applying induction on $G\left[\pi_{\alpha+1}, \ldots, \pi_{n}\right]$. If $\pi_{\beta} \pi_{\alpha} \notin E(G)$, we know that $\pi_{\beta}$ has at least $2 k$ neighbors among $\left\{\pi_{\alpha+1}, \ldots, \pi_{n}\right\}$ and so we can find $t$ such that $t \pi_{\beta} \in E(G)$ and $t \in\left\{\pi_{\alpha+1}, \ldots, \pi_{n}\right\} \backslash S$. Applying the induction hypothesis 
on $H=G\left[\pi_{\alpha}, \ldots, \pi_{n}\right]$, we see that $H$ has a pseudo-path system $\mathscr{P}$ rooted at $\left\{\left(s_{i}, t_{i}\right): i \in[k] \backslash[q]\right\} \cup$ $\left\{\left(\pi_{\alpha}, t\right)\right\}$. Replacing the $\pi_{\alpha}, t$-path in $\mathscr{P}$ by the cycle obtained from this path by adjoining the path $Q$ yields a new pseudo-path system $\mathscr{P}^{\prime}$. It is obvious that $\mathscr{P}^{\prime} \cup\left\{P_{i}: i \in[q-1]\right\}$ then becomes a required pseudo-path system for $G$.

CASE 2.2. There exists $i \in[q]$ such that $\pi_{\alpha}=s_{i} \neq t_{i}$.

For each $i \in[q]$ with $s_{i}=t_{i}=\pi_{\alpha}$, we can choose the path of length zero consisting of the single vertex $\pi_{\alpha}$. In this way, our consideration can be reduced to the case that $s_{i}=\pi_{\alpha} \neq t_{i}$ for all $i \in[q]$.

CASE 2.2.1. $n>\alpha>n-2 k$.

This means that $G\left[\pi_{\alpha}, \ldots, \pi_{n}\right]$ is a complete graph and so must be rooted $k$-connected. Accordingly, we can find an $s_{i}, t_{i}$-pseudo-path $P_{i}^{\prime}$ for each $i \in[k]$ such that the inner vertices of these $P_{i}^{\prime}$ form a partition of $\left\{\pi_{\alpha}, \ldots, \pi_{n}\right\} \backslash S$. Because $\pi$ is $(2 k+1)$-thick, we can now deduce that $N_{G}\left(\pi_{\beta}\right) \supseteq\left\{\pi_{\alpha+1}, \ldots, \pi_{n}\right\}$. Replacing the vertex $\pi_{\alpha}$ by the path $Q$ in $P_{1}^{\prime}$ and keeping those $P_{i}^{\prime}$ for $i \geq 2$ unchanged, we get the claimed $k$ pseudo-paths.

CASE 2.2.2. $\alpha \leq n-2 k$.

Since $\pi$ is a $(2 k+1)$-thick Hamiltonian vertex ordering of $G$ while $\left|\left\{\pi_{\alpha+1}, \ldots, \pi_{n}\right\}\right| \geq 2 k$, we can choose a set $W=\left\{s_{1}^{\prime}, \ldots, s_{q}^{\prime}\right\}$ of $q$ different vertices of $\left\{\pi_{\alpha+1}, \ldots, \pi_{n}\right\} \backslash S$ such that $s_{1}^{\prime} \pi_{\beta} \in E(G)$ and $W \backslash\left\{s_{1}^{\prime}\right\} \subseteq N_{G}\left(\pi_{\alpha}\right)$. By the induction assumption, $G\left[\pi_{\alpha+1}, \ldots, \pi_{n}\right]$ has a pseudo-path system $\mathscr{P}$ rooted at $\left\{\left(s_{i}^{\prime}, t_{i}\right): i \in[q]\right\} \cup\left\{\left(s_{i}, t_{i}\right): i \in[k] \backslash[q]\right\}$. Replacing the unique $s_{1}^{\prime}, t_{1}$-path in $\mathscr{P}$, say $P_{1}^{\prime}$, by the path obtained from $Q$ and $P_{1}^{\prime}$ by connecting them at $s_{1}^{\prime} \pi_{\beta}$ and replacing the unique $s_{i}^{\prime}, t_{i}$-path in $\mathscr{P}$ for each $i \in[q] \backslash\{1\}$, say $P_{i}^{\prime}$, by the path obtained from $P_{i}^{\prime}$ by adding the additional edge $\pi_{\alpha} s_{i}^{\prime}$, we derive the required pseudo-path system of $G$.

Proof of Corollary 14: Take $\pi_{1} \cdots \pi_{k} \in V(G)^{\underline{k}}$. As $2 k-1=2(k-1)+1$, Theorem 13 says that $G$ has a spanning $(k-1)$-path system rooted at $\left\{\left(\pi_{i}, \pi_{i+1}\right): i \in[k-1]\right\}$. Amalgamating the $k-1$ paths in this path system gives rise to a Hamiltonian path passing through $\pi_{1}, \ldots, \pi_{k}$ in this order, as wanted.

\subsubsection{Proofs of Theorems 15, 16, 17, 18, 19 and 21}

Lemma 100 For every graph $G$ and every positive integer $n$, it holds $\operatorname{sc}\left(G \bigvee K_{n}\right)=\operatorname{sc}(G)-n$.

Proof: When $G$ is a complete graph, the result is trivial. When $G$ is not a complete graph, adding a universal vertex to $G$ will cause the scattering number decrease by 1 as this universal vertex will appear in any scattering set of the new graph, which completes the proof.

Proof of Theorem 15: Note that $\operatorname{sc}\left(K_{n}\right)+\mathcal{H}\left(K_{n}\right)=\operatorname{sc}\left(K_{n}\right)+\kappa\left(K_{n}\right)=(3-n)+(n-1)=2$. So, we may assume below that $G$ is not a complete graph.

Choose a set $S \in\left(\begin{array}{c}V(G) \\ \kappa(G)\end{array}\right)$ fulfilling c $(G-S) \geq 2$. We then see that $\mathrm{sc}(G) \geq \mathrm{c}(G-S)-|S| \geq 2-\kappa(G)$, as desired.

It remains to show $\operatorname{sc}(G)+\mathcal{H}(G) \leq 2$. By Lemmas 99 and 100 we can and will assume that $\mathcal{H}(G)=$ $k \geq 1$. As a result of Theorem 7. the deletion from $G$ of any vertex subset of size at most $k-1$ results in a traceable graph and $\operatorname{sosc}(G) \leq 2-k=2-\mathcal{H}(G)$ follows. 
Proof of Theorem 16: Pick $G \in \mathcal{C}$. If $\operatorname{sc}(G) \leq 1$, we will have $\mathcal{H}(G) \geq 1$ and so, according to the definition of $\mathcal{H}(G), G$ is traceable. If $\operatorname{sc}(G) \leq 0$, we will have $\mathcal{H}(G) \geq 2$ and so, by Theorem $1 . G$ is Hamiltonian. If $\operatorname{sc}(G) \leq-1$, we will have $\mathcal{H}(G) \geq 3$ and so, taking Theorem 9 into consideration, $G$ is Hamiltonian-connected. This finishes the proof of the first three readings.

We now assume that $|V(G)| \geq 3, G-v$ is traceable, and hence $\mathcal{H}(G-v) \geq 1$, for every $v \in V(G)$, and try to show that $G$ is Hamiltonian. Firstly, the assumption implies the connectedness of $G$ and so Eq. (6) is valid. But Theorem 15 asserts

$$
\max _{v \in V(G)} \operatorname{sc}(G-v) \leq 2-\min _{v \in V(G)} \mathcal{H}(G-v) \leq 1 .
$$

Therefore, Eq. (6) now yields $\operatorname{sc}(G) \leq 0$ and then the assumption of $\operatorname{sc}(G)+\mathcal{H}(G)=2$ leads to $\mathcal{H}(G) \geq 2$. Finally, it follows from Theorem 5 that $G$ is Hamiltonian, completing the proof of iv).

The following proof of Theorem 17 indeed suggests an algorithm to find an optimal path cover of a forest. The algorithm is basically the same with the one presented by Franzblau and Raychaudhuri (36, Lemma 2, Lemma 3).

Proof of Theorem 17; Since a forest has no cycle, Theorem 1 asserts that $\mathcal{H}(G) \leq 1$. Making use of Eqs. (1) and (2), our task is to prove

$$
\pi(G) \leq \operatorname{sc}(G) .
$$

For any two graphs $H_{1}$ and $H_{2}$, it holds $\pi\left(H_{1} \cup H_{2}\right)=\pi\left(H_{1}\right)+\pi\left(H_{2}\right)$ and $\operatorname{sc}\left(H_{1} \cup H_{2}\right) \geq \operatorname{sc}\left(H_{1}\right)+$ $\operatorname{sc}\left(H_{2}\right)$. Therefore, we may even assume that $G$ is connected. Let $H$ be the minimal subtree of $G$ which contains all vertices of $G$ with degree at least 3 . If $G$ is a path (and hence $H$ is empty), we can easily verify Eq. (19). Otherwise, take $v \in V(H)$ which is either the only vertex of $H$ or a leaf of $H$. We can enumerate the components of $G-v$ as $G^{\prime}, P_{1}, \ldots, P_{s}$, where $s \geq 2$, and $G\left[P_{i} \cup\{v\}\right]$ are all paths for $i \in[s]$. Let $P$ be the path $G\left[P_{1} \cup\{v\} \cup P_{2}\right]$. Note that $\operatorname{sc}(G) \geq \operatorname{sc}(G-P)+\operatorname{sc}(P)=\operatorname{sc}(G-P)+1$ and $\pi(G) \leq \pi(G-P)+\pi(P)=\pi(G-P)+1$. The induction hypothesis gives $\pi(G-P) \leq \operatorname{sc}(G-P)$ and so Eq. 19 follows, as desired.

Proof of Theorem 18: We assume that $|V(G)|=n$. Consider the function $f$ of a real variable such that $f(x)=x(n-x)+\frac{x(x-1)}{2}$ for $x \in \mathbb{R}$. It is clear that

$$
f(\operatorname{dgn}(G)) \geq|E(G)| \geq f(\mathcal{H}(G)),
$$

where the first inequality already appeared in Lick and White (75, Proposition 3). Note that

$$
\max \{\operatorname{dgn}(G), \mathcal{H}(G)\} \leq n-1
$$

while $f(x)$ is an increasing function for $x \in\left(-\infty, n-\frac{1}{2}\right]$. This demonstrates

$$
\operatorname{dgn}(G) \geq \mathcal{H}(G) .
$$

If $G$ has an exact- $k$-thick Hamiltonian vertex ordering $\pi$, we surely have $\mathcal{H}(G) \geq k$ and $|E(G)|=$ $f(k)$. As $f(x)$ is an increasing function when $x<n-\frac{1}{2}$, further recalling $|E(G)| \geq f(\mathcal{H}(G)$ ) (Eq. (20), we get to

$$
\mathcal{H}(G)=k .
$$


Note that in the reversal of $\pi$, each vertex has at most $k$ neighbors appeared earlier. This says that $\operatorname{dgn}(G) \leq k$. This along with Eqs. 21] and 221 proves that $\operatorname{dgn}(G)=\mathcal{H}(G)=k$.

Conversely, let us assume that $\operatorname{dgn}(G)=\mathcal{H}(G)=k$. In light of Eq. 200, it holds

$$
|E(G)|=f(\mathcal{H}(G))=f(k) .
$$

Let $\pi$ be a $k$-thick Hamiltonian vertex ordering of $G$. According to Eq. 23), we see that $\pi$ is indeed an exact- $k$-thick Hamiltonian vertex ordering of $G$, showing that $G$ is an exact- $k$-thick Hamiltonian graph.

By Eq. (4) and Theorem 15, we have

$$
\operatorname{sc}(G)+\mathcal{H}(G) \leq 2 \leq \operatorname{sc}(G)+\kappa(G) \leq \operatorname{sc}(G)+\operatorname{dgn}(G) .
$$

Consequently, the relation $\operatorname{dgn}(G)=\mathcal{H}(G)$ will force $2-\operatorname{sc}(G)=\mathcal{H}(G)=\kappa(G)=\operatorname{dgn}(G)$, as desired.

Proof of Theorem 19: To prove the result, we need only consider the case that $G$ is connected. Suppose that we pick a simplicial vertex $v$ and a neighbor $u$ of $v$ in $G$. If there is any path containing $v$, we can add $u$ into it to make a new path where $u$ is adjacent to $v$ in the new path. Thus, $G$ has to be traceable or Hamiltonian provided $G-u$ is traceable or Hamiltonian, respectively. This consideration easily proves that $G$ is neither hypotraceable nor hypohamiltonian.

We now show that $G$ cannot be hypo-Hamiltonian-connected. Given any two vertices $w_{1}$ and $w_{2}$ of $G$, we need to verify that $G$ has a spanning $w_{1}, w_{2}$-path provided $G-u$ is Hamiltonian-connected for every $u \in V(G)$. If $G$ is a complete graph, the result is trivial. So, we assume that $G$ has a clique tree with at least two leaves, say $Q_{1}$ and $Q_{2}$. As discussed above, we only need to find a vertex $u \notin\left\{w_{1}, w_{2}\right\}$ which is a neighbor of a simplicial vertex in $G$. Let $S$ be the set of nonsimplicial vertices of $G$ that are contained in $Q_{1}$. If $|S|=1$, then deleting the only vertex in $S$ will disconnect the graph, a contradiction. If $S=\left\{x_{1}, x_{2}\right\}$, we can find that $G-x_{1}$ does not contain any Hamiltonian path with $x_{2}$ as one endpoint, a contradiction again. Therefore, we see that $|S| \geq 3$ and so any vertex in $S \backslash\left\{w_{1}, w_{2}\right\}$ can be chosen as $u$, finishing the proof.

Proof of Theorem 21: a) If $\mathcal{H}(G)=2$, the result follows from Corollary 2. We now assume that $\mathcal{H}(G) \leq 1$ and so, by Eq. (2), we can take a path cover of $G$ of size $\pi(G)=2-\mathcal{H}(G)$ in which the vertex $v$ appears on a path $P$. The path $P$ can be split into two disjoint paths where $v$ is the endpoint of one of them and thus we get a path cover of size at most $2-\mathcal{H}(G)+1$, showing that $\mathcal{H}_{G}(v) \in\{\mathcal{H}(G), \mathcal{H}(G)-1\}$, as desired.

b) Deleting an edge from a Hamiltonian graph surely yields a traceable graph. Thus, Theorem 1 settles the case of $\mathcal{H}(G)=2$. If $\mathcal{H}(G) \leq 1$, Eq. (2) implies that $G$ has a path cover of size $\pi(G)=2-\mathcal{H}(G)$. Let $\mathcal{E}$ be the set of edges appeared in this path cover and let $\mathcal{E}^{\prime}=\mathcal{E} \backslash\{e\}$. As $G\left[\mathcal{E}^{\prime}\right]$ corresponds to a path cover of $G-e$ of size at most $\pi(G)+1$, we use Eq. (2) again to deduce that $\mathcal{H}(G-e) \geq \mathcal{H}(G)-1$, completing the proof. 


\subsection{Interval graphs}

\subsubsection{Preliminaries}

A simple vertex in a graph is a simplicial vertex whose neighbors have closed neighborhoods that are linearly ordered by inclusion. As defined by Farber (35), a graph is strongly chordal if and only if every one of its induced subgraphs has a simple vertex.

Lemma 101 Let $G$ be a traceable graph. Let $v$ be a simple vertex of $G$ and let $w$ be a neighbor of $v$ in $G$ such that

$$
N_{G}[w]=\cap_{w^{\prime} \in N_{G}(v)} N_{G}\left[w^{\prime}\right] .
$$

Then both $G-v$ and $G-\{v, w\}$ are traceable.

Proof: Suppose that $P=\left(x_{1}, \ldots, x_{k}, v, y_{\ell}, \ldots, y_{1}\right)$ is a Hamiltonian path of $G$. It is easy to see that $\left(x_{1}\right.$, $\left.\ldots, x_{k}, y_{\ell}, \ldots, y_{1}\right)$ is a Hamiltonian path of $G-v$.

We next turn to show that $G-\{v, w\}$ is traceable. To this end, it suffices to find a neighbor $u$ of $v$ in $G$ such that $G-\{v, u\}$ is traceable, as Eq. (24) says that we can derive a Hamiltonian path of $G-\{v, w\}$ by replacing possibly $w$ with $u$ in a Hamiltonian path of $G-\{v, u\}$.

If $v$ is an endpoint of $P$, say $k=0$, then we can choose $u$ to be $y_{\ell}$. In case that $v$ is not an endpoint of $P$, since $v$ is simple, we may assume that $N_{G}\left[x_{k}\right] \subseteq N_{G}\left[y_{\ell}\right]$, we can choose $u=x_{k}$ and check that $\left(x_{1}, x_{2}, \ldots, x_{k-1}, y_{\ell}, y_{\ell-1}, \ldots, y_{1}\right)$ is a Hamiltonian path of $G-\left\{v, x_{k}\right\}$.

The following lemma and its generalizations appear in Arikati and Pandu Rangan (3), Damaschke (29), Hung and Chang (54), Isaak (57), Manacher et al. (80). It bears a striking resemblance to Damaschke et al. (30, Theorem 3).

Lemma 102 If an interval graph $G$ is traceable, then every normal vertex ordering of $G$ is a Hamiltonian vertex ordering.

Proof: Setting $n=|V(G)|$, we only need to consider the case of $n \geq 3$. Let $\pi_{1} \cdots \pi_{n}$ be a normal vertex ordering of $G$ with respect to an interval representation $\mathcal{I}$ and we intend to show that $\mathcal{H}_{G}(\pi) \geq 1$. Note that the connectedness of $G$ gives $\pi_{1} \pi_{2} \in E(G)$. We also check that $\pi_{1}$ is a simple vertex of $G$ and hence an application of Lemma 101 yields that both $G-\pi_{1}$ and $G-\left\{\pi_{1}, \pi_{2}\right\}$ are traceable.

If $r_{\mathcal{I}}\left(\pi_{2}\right)=\min \left\{r_{\mathcal{I}}\left(\pi_{i}\right): i \in\{2, \ldots, n\}\right\}$, then $\pi_{2} \cdots \pi_{n}$ is a normal vertex ordering of $G-\pi_{1}$. By the induction hypothesis, $\left(\pi_{2}, \ldots, \pi_{n}\right)$ is a Hamiltonian path of $G-\pi_{1}$, which implies that $\left(\pi_{1}, \ldots, \pi_{n}\right)$ is a Hamiltonian path of $G$.

Else, we have $\pi_{3} \pi_{1} \notin E(G), \pi_{3} \pi_{2} \in E(G)$ and $r_{\mathcal{I}}\left(\pi_{3}\right)=\min \left\{r_{\mathcal{I}}\left(\pi_{i}\right): i \in\{2, \ldots, n\}\right\}$. This says that $\pi_{3} \cdots \pi_{n}$ is a normal vertex ordering of $G-\left\{\pi_{1}, \pi_{2}\right\}$ and so the induction assumption guarantees that $\left(\pi_{3}, \ldots, \pi_{n}\right)$ is a Hamiltonian path of $G-\left\{\pi_{1}, \pi_{2}\right\}$. It is now immediate that $\left(\pi_{1}, \ldots, \pi_{n}\right)$ is a Hamiltonian path of $G$, finishing the proof.

Lemma 103 Manacher et al. (80. Lemma 2) Let $\pi$ be an almost normal vertex ordering of an $n$-vertex interval graph $G$ with respect to an interval representation $\mathcal{I}$. For any $i, j>1$, if $\ell_{\mathcal{I}}\left(\pi_{i}\right)<\ell_{\mathcal{I}}\left(\pi_{j}\right)$ and $r_{\mathcal{I}}\left(\pi_{i}\right)<r_{\mathcal{I}}\left(\pi_{j}\right)$, then $i<j$. In addition, if $\pi_{n} \pi_{n-1} \in E(G)$, then $\pi_{n}$ and $\pi_{n-1}$ belong to the rightmost clique of $G$ with respect to $\mathcal{I}$. 
Proof: We aim to derive a contradiction under the assumption of $i>j$. Since $\pi$ is an almost normal vertex ordering, the assumption of $i>j$ together with $r_{\mathcal{I}}\left(\pi_{i}\right)<r_{\mathcal{I}}\left(\pi_{j}\right)$ entails $\pi_{j-1} \pi_{j} \in E(G)$ and $\pi_{j-1} \pi_{i} \notin E(G)$. It is impossible that $r_{\mathcal{I}}\left(\pi_{j-1}\right)<\ell_{\mathcal{I}}\left(\pi_{i}\right)$ as it implies $r_{\mathcal{I}}\left(\pi_{j-1}\right)<\ell_{\mathcal{I}}\left(\pi_{i}\right)<\ell_{\mathcal{I}}\left(\pi_{j}\right)$ and so $\pi_{j-1} \pi_{j} \notin E(G)$. We now obtain from $\pi_{j-1} \pi_{i} \notin E(G)$ that $r_{\mathcal{I}}\left(\pi_{i}\right)<\ell_{\mathcal{I}}\left(\pi_{j-1}\right)$. We can continue like this to get $r_{\mathcal{I}}\left(\pi_{i}\right)<\ell_{\mathcal{I}}\left(\pi_{t}\right)$ for $t=j-2, \ldots, 1$. This contradicts the fact that $\pi_{1}$ appears in the leftmost clique in the given interval representation $\mathcal{I}$ of $G$.

To prove the second reading, it suffices to prove that there is no $k$ such that $r_{\mathcal{I}}\left(\pi_{n-1}\right)<\ell_{\mathcal{I}}\left(\pi_{k}\right)$ and there is no $k^{\prime}$ such that $r_{\mathcal{I}}\left(\pi_{n}\right)<\ell_{\mathcal{I}}\left(\pi_{k^{\prime}}\right)$. If either such $k$ or such $k^{\prime}$ exists, from the first assertion in the lemma we deduce either $k=n$ or $k^{\prime}>n$, respectively, both of which being absurd.

Lemma 104 Let $\mathcal{I}$ be an interval representation of a graph $G$. Let a and b be two different vertices of $G$ such that $r_{\mathcal{I}}(a)=\min \left\{r_{\mathcal{I}}(v): v \in V(G)\right\}$ and $\ell_{\mathcal{I}}(b)=\max \left\{\ell_{\mathcal{I}}(v): v \in V(G)\right\}$. Then $G$ is traceable if and only if $G$ has a Hamiltonian path which starts at $a$ and ends at $b$.

Proof: Only the forward direction needs to be addressed. We define an interval representation $\mathcal{I}^{\prime}$ of $G$ by setting $\mathcal{I}^{\prime}(b)=\left[\ell_{\mathcal{I}}(b)+1, \ell_{\mathcal{I}}(b)+2\right], \mathcal{I}^{\prime}(w)=\left[\ell_{\mathcal{I}}(w), \ell_{\mathcal{I}}(b)+1\right]$ for $w \in N_{G}(b)$ and $\mathcal{I}^{\prime}(z)=\mathcal{I}(z)$ for $z \notin N_{G}[b]$. By Lemma 102, each ordering $\pi$ constructed by the normal path algorithm $\operatorname{NP}\left(G, \mathcal{I}^{\prime}, a\right)$ is a Hamiltonian vertex ordering. Notice that $\ell_{\mathcal{I}^{\prime}}(z)<\ell_{\mathcal{I}^{\prime}}(b)$ and $r_{\mathcal{I}^{\prime}}(z)<r_{\mathcal{I}^{\prime}}(b)$ for every $z \in V(G) \backslash\{b\}$. It then follows from Lemma 103 that $\pi_{|V(G)|}=b$ and hence $\pi$ is a Hamiltonian vertex ordering of $V(G)$ which starts at $a$ and ends at $b$, completing the proof.

Lemma 105 Suppose that $G$ is a graph and $\mathcal{I}$ is one of its interval representations. Let $a$ and $b$ be a leftmost vertex and a rightmost vertex of $G$, respectively, with respect to the interval representation $\mathcal{I}$. Suppose that $G$ contains an a, b-path $P$ and $V(G) \backslash V(P)$ is the disjoint union of $T_{1}, \ldots, T_{\ell}, Q_{1}, \ldots, Q_{m}$ such that $G\left[T_{i} \cup\{a\}\right]$ contains a spanning path starting from a for each $i \in[\ell]$ and $G\left[Q_{i} \cup\{b\}\right]$ contains a spanning path starting from bor each $i \in[m]$. Then $G$ possesses a spanning $a, b$-path.

Proof: It is clear that $G\left[V(P) \cup T_{1}\right]$ contains a Hamiltonian path. In light of Lemma 104, $G\left[V(P) \cup T_{1}\right]$ has a Hamiltonian path $P^{\prime}$ connecting $a$ to $b$. Repeating this argument eventually absorbs all vertices on $T_{2}, \ldots, T_{\ell}, Q_{1}, \ldots, Q_{m}$ one by one into $P^{\prime}$ and thus yields a required Hamiltonian path of $G$.

The next result implies that every cocomparability graph is AT-free and so, naturally, the idea of its proof should be in the folklore.

Lemma 106 Let $G$ be the cocomparability graph of a poset $(S,<)$. Let $a, b, c$ be three elements of $V(G)$ and take an $a, b$-path $P$ in $G$. If $N_{G}[c] \cap V(P)=\emptyset$, then either $a<c, b<c$ or $c<a, c<b$.

Proof: Let $P=\left(v_{1}, \ldots, v_{k}\right)$. The assumption of $N_{G}[c] \cap V(P)=\emptyset$ means that $c$ is comparable with every vertex on $P$ in the poset. If there exists $v_{i}<c$ and $c<v_{j}$, then we can find integers $t$ and $t+1$ such that $v_{t}<c<v_{t+1}$ or $v_{t+1}<c<v_{t}$ and hence $v_{t} v_{t+1} \notin E(G)$, arriving at a contradiction. 


\subsubsection{Proof of Theorem 22}

To present a proof of Theorem 22, our first main result for interval graphs, we have to prepare three more lemmas.

Lemma 107 Let $\mathcal{I}$ be an interval representation of a graph $G$ and let a and $b$ be two vertices such that $r_{\mathcal{I}}(a)=\min \left\{r_{\mathcal{I}}(z): z \in V(G)\right\}$ and $\ell_{\mathcal{I}}(b)=\max \left\{\ell_{\mathcal{I}}(z): z \in V(G)\right\}$. Let $k$ be a positive integer and suppose that $G$ has a spanning $k$-rail between a and $b$. Take a subset $T$ of $N_{G}(a) \backslash\{b\}$ such that $|T| \leq k$. Then $G$ has a typical $k$-rail consisting of $k$ paths $P_{1}, \ldots, P_{k}$ such that $\left|V\left(P_{i}\right) \cap T\right| \leq 1$ for $i \in[k]$.

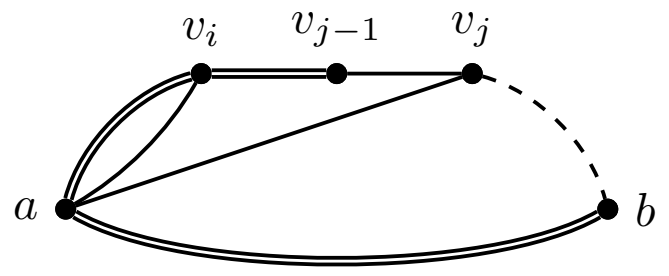

(a) Two neighbors of $a$ on one path.

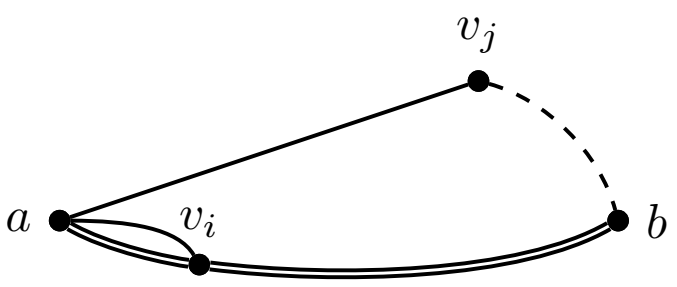

(b) Rearranging the two paths.

Fig. 6: Distributing neighbors to different paths in the proof of Lemma 107 .

Proof: The idea of the proof is depicted in Fig. 6. If the given spanning $k$-rail $\mathcal{T}$ between $a$ and $b$ does not satisfy the requirement, we will find two paths $Q_{1}=\left(a=v_{1}, v_{2}, \ldots, v_{m}=b\right)$ and $Q_{2}=(a=$ $\left.u_{1}, u_{2}, \ldots, u_{\ell}=b\right)$ among the $k$ paths of $\mathcal{T}$ such that $\left\{u_{2}, \ldots, u_{\ell-1}\right\} \cap T=\emptyset$ and there exist two indexes $i$ and $j$ such that $1<i<j<m$ and $v_{i}, v_{j} \in T$. We focus on the path $Q=\left(v_{j-1}, \ldots, v_{1}=a=\right.$ $\left.u_{1}, u_{2}, \ldots, u_{\ell}=b\right)$. In view of Lemma 104 , there is an $a, b$-path $Q_{2}^{\prime}$ whose set of vertices is the same as that of $Q$. Let $Q_{1}^{\prime}$ be the path $\left(a, v_{j}, v_{j+1}, \ldots, v_{m}=b\right)$. Now replace $Q_{1}$ and $Q_{2}$ by $Q_{1}^{\prime}$ and $Q_{2}^{\prime}$ in $\mathcal{T}$ to get a new spanning $k$-rail. We continue this process and will finally reach a desired typical $k$-rail of $G$.

Lemma 108 Let $G$ be a graph and $H$ a subgraph of $G$. Let $\mathcal{I}$ be an interval representation of $H$ and let $\widetilde{a}$ and $b$ be two vertices of $H$ such that $r_{\mathcal{I}}(\widetilde{a})=\min \left\{r_{\mathcal{I}}(z): z \in V(H)\right\}$ and $\ell_{\mathcal{I}}(b)=\max \left\{\ell_{\mathcal{I}}(z)\right.$ : $z \in V(H)\}$. Let $k$ be a positive integer and suppose that $H$ has a spanning $k$-rail between $\widetilde{a}$ and $b$. Take $a \in V(G) \backslash V(H)$ such that $a b \notin E(G)$ and $\left|N_{G}(a) \cap N_{H}(\widetilde{a})\right| \geq k-1$. Further assume that $G-(V(H) \backslash\{\widetilde{a}\})$ has a spanning $a, \widetilde{a}$-path $P$. Then $G$ has a spanning $k$-rail between $a$ and $b$.

Proof: In view of $a b \notin E(G)$ and $\left|N_{G}(a) \cap N_{H}(\widetilde{a})\right| \geq k-1$, it follows from Lemma 107 that we can assume that $H$ has a spanning $k$-rail between $\widetilde{a}$ and $b$ consisting of $P_{1}, \ldots, P_{k}$ such that for each $i \in[k-1], P_{i}$ contains a neighbor of $a$, say $v_{i}$, as an inner vertex. For each $i \in[k-1]$, let the subpath of $P_{i}$ from $v_{i}$ to $b$ be $Q_{i}$. Since $a v_{i} \in E(G)$, to obtain the asserted $k$-rail, we only need to find a Hamiltonian path from $a$ to $b$ in $G-\left(\cup_{i \in[k-1]} V\left(Q_{i}\right) \backslash\{b\}\right)$. Due to the existence of the path $P$, it remains to find a Hamiltonian path from $\widetilde{a}$ to $b$ in $H-\left(\cup_{i \in[k-1]} V\left(Q_{i}\right) \backslash\{b\}\right)$, which is guaranteed by Lemma 105 , ending the proof. 
Lemma 109 Let $k$ be a positive integer and let $G$ be an interval graph. If $G$ has a typical $(k+1)$-rail, then it has a typical $k$-rail.

Proof: This is direct from Lemma 105

It is now time to reap the benefits of our long sowing work.

Proof of Theorem 22, Let $n=|V(G)|$. To prove the theorem, let us establish the following string of implications.

a) $\Rightarrow$ b) This is straightforward.

b) $\Rightarrow$ c) Suppose that $\mathcal{I}$ is an interval representation of $G$ and $a, b$ are two vertices of $G$ such that $r_{\mathcal{I}}(a)=$ $\min \left\{r_{\mathcal{I}}(z): z \in V(G)\right\}$ and $\ell_{\mathcal{I}}(b)=\max \left\{\ell_{\mathcal{I}}(z): z \in V(G)\right\}$. Further suppose that $P_{1}, \ldots, P_{k}$ are $k$ distinct pairwise-internally-disjoint $a, b$-paths which cover $V(G)$. Let $P_{i}^{\prime}=V\left(P_{i}\right) \backslash\{a, b\}$ for every $i \in[k]$.

If $a b \in E(G)$, then $G$ is a complete graph and so the result is trivial. In the sequel, we suppose that $a b \notin E(G)$ and hence $P_{i}^{\prime} \neq \emptyset$ for every $i \in[k]$.

We shall proceed by induction on $k$. If $k=1$, there is nothing to prove. Suppose $k>1$ and the claim holds for each smaller $k$. Let $Z$ be a set of at most $k-1$ distinct vertices and we intend to show that $G-Z$ is traceable. We separate two cases.

CASE 1. $\{a, b\} \cap Z \neq \emptyset$.

In this case, $Z^{\prime}=Z \backslash\{a, b\}$ contains less than or equal to $k-2$ vertices. Recall from Lemma 109 that $G$ has a typical $(k-1)$-rail. Consequently, our induction hypothesis says that $G-Z^{\prime}$ is traceable. By Lemma 104. $G-Z^{\prime}$ has a spanning $a, b$-path, from which we can deduce that $G-Z$ is traceable.

CASE 2. $\{a, b\} \cap Z=\emptyset$.

If $Z=\emptyset$, we can get the result by invoking Lemma 109 . We now turn to the case of $Z \neq \emptyset$. Take $z \in Z$ and set $Z^{\prime}=Z \backslash\{z\}$. Without loss of generality, we assume that $V\left(P_{1}\right) \cap Z=\emptyset$. Note that $G^{\prime}=G-P_{1}^{\prime}$ has a spanning $(k-1)$-rail connecting the opposite pair $a$ and $b$. By the induction hypothesis, this implies that $G^{\prime}-Z^{\prime}$ has a Hamiltonian path. Lemma 104 then asserts that $G^{\prime}-Z^{\prime}$ has a Hamiltonian path connecting $a$ and $b$, say $\sigma$. Suppose $\sigma=\left(a=\sigma_{1}, \sigma_{2}, \ldots, \sigma_{h}=b\right), z=\sigma_{j}, 1<j<h$, and $P_{1}=(a=$ $\left.\beta_{1}, \beta_{2}, \ldots, \beta_{h^{\prime}}=b\right)$. Then $\left(\sigma_{j+1}, \ldots, \sigma_{h}=b=\beta_{h^{\prime}}, \beta_{h^{\prime}-1}, \beta_{h^{\prime}-2}, \ldots, \beta_{1}=a=\sigma_{1}, \sigma_{2}, \ldots, \sigma_{j-1}\right)$ is a Hamiltonian path of $G-Z$, completing the proof.

c) $\Rightarrow$ d) Trivial.

d) $\Rightarrow$ e) Let $\mathcal{I}$ be an interval representation of $G$, let $a$ be a leftmost vertex of $G$ with respect to $\mathcal{I}$, and let $\pi$ be an output of $\operatorname{NP}(G, \mathcal{I}, a)$. By the rule of the normal path algorithm, we see that a $k$-thick normal vertex ordering must be a Hamiltonian vertex ordering. It hence suffices to show that $\pi$ is $k$-thick.

We first exhibit that $\mathrm{d}_{G, \pi}(i) \geq k$ for each $i \in[n-k]$. If there exists $i \in[n-k]$ such that $\mathrm{d}_{G, \pi}(i)<k$, then there exists $S \in\left(\begin{array}{c}\left\{\pi_{i+1}, \ldots, \pi_{n}\right\} \\ k-1\end{array}\right)$ such that $N_{G}\left(\pi_{i}\right) \cap\left\{\pi_{i+1}, \ldots, \pi_{n}\right\} \subseteq S$. Letting $G^{\prime}=G-S$, we can choose an output of $\operatorname{NP}\left(G^{\prime},\left.\mathcal{I}\right|_{V\left(G^{\prime}\right)}, a\right)$, say $\xi$, such that $\xi_{p}=\pi_{p}$ for all $p \in[i]$. It is clear that $\xi_{i} \xi_{i+1} \notin E(G)$ and so $\xi$ is not a Hamiltonian vertex ordering of $G^{\prime}$. According to Lemma 102 , this means that $G^{\prime}$ is not traceable, contradicting statement d).

Our remaining task is to show that $G\left[\pi_{n-k+1}, \ldots, \pi_{n}\right]$ is a complete graph. If $k=n-1$, it follows from d) that $G=K_{k+1}$ and thus we are done. Assume now $k<n-1$. If $G\left[\pi_{n-k+1}, \ldots, \pi_{n}\right]$ were not 
a complete graph, then we can get two vertices $u$ and $w$ from $\left\{\pi_{n-k+1}, \ldots, \pi_{n}\right\}$ such that $u w \notin E(G)$. Without loss of generality, suppose that

$$
r_{\mathcal{I}}(u)<\ell_{\mathcal{I}}(w)
$$

Remember that $\mathrm{d}_{G, \pi}(n-k) \geq k$ and so

$$
N_{G}\left(\pi_{n-k}\right) \supseteq\left\{\pi_{n-k+1}, \ldots, \pi_{n}\right\} .
$$

Eq. 26], combined with Eq. 25], gives $r_{\mathcal{I}}(u)<r_{\mathcal{I}}\left(\pi_{n-k}\right)$. This and the rule of the normal path algorithm then lead to

$$
\pi_{n-k-1} u \notin E(G) \text {. }
$$

As we knew before that $\mathrm{d}_{G, \pi}(n-k-1) \geq k$, Eq. 27] implies

$$
\pi_{n-k-1} w \in E(G) \text {. }
$$

As a result of Eqs. 25), 27) and (28), it holds $r_{\mathcal{I}}(u)<\ell_{\mathcal{I}}\left(\pi_{n-k-1}\right)$. This leads to a contradiction with Lemma 103 .

e) $\Rightarrow \mathbf{f}) \Rightarrow \mathbf{g} \quad$ This is obvious.

g) $\Rightarrow$ a) There is nothing to prove when $n \leq 2$. We now consider the case of $n \geq 3$ under the hypothesis that the result holds for smaller $n$.

Suppose that $\mathcal{I}$ is an interval representation of $G$ and $a, b$ form a pair of opposite vertices of $G$ with respect to $\mathcal{I}$, say $r_{\mathcal{I}}(a)=\min \left\{r_{\mathcal{I}}(z): z \in V(G)\right\}$ and $\ell_{\mathcal{I}}(b)=\max \left\{\ell_{\mathcal{I}}(z): z \in V(G)\right\}$. We also assume that $G$ has a $k$-thick Hamiltonian vertex ordering $\pi_{1} \cdots \pi_{n}$. Our task is to establish the existence of a spanning $k$-rail of $G$ between $a$ and $b$. Note that we may assume that $a=\pi_{i}, b=\pi_{j}$ and $i<j$, as otherwise we may simply change the interval representation $\mathcal{I}$ to be $-\mathcal{I}$. We may also assume that $a b \notin E(G)$, as otherwise $G$ is complete and then the result is trivial.

CASE $1 . \quad i>1$.

Let $H=G\left[\pi_{i}, \ldots, \pi_{n}\right]$. From $a b \notin E(G)$ we can derive that $\pi_{i} \cdots \pi_{n}$ is a $k$-thick Hamiltonian vertex ordering of $H$. It then follows from the induction hypothesis that $H$ has a spanning $k$-rail between $a$ and $b$, which consists of $k$ paths, say $P_{1}, \ldots, P_{k}$. Due to the existence of the path $\left(\pi_{1}, \ldots, \pi_{i}\right) \pi_{i} P_{1}$, we deduce from Lemma 104 that $G\left[V\left(P_{1}\right) \cup\left\{\pi_{1}, \ldots, \pi_{i-1}\right\}\right]$ has a spanning $a, b$-path $P_{1}^{\prime}$. It is clear that $P_{1}^{\prime}, P_{2}, \ldots, P_{k}$ form a spanning $k$-rail of $G$ between $a$ and $b$.

CASE 2. $i=1$.

Let $\widetilde{a}$ be the vertex from $V(G) \backslash\left\{\pi_{1}\right\}$ such that $r_{\mathcal{I}}(\widetilde{a})=\min \left\{r_{\mathcal{I}}(z): z \in V(G) \backslash\left\{\pi_{1}\right\}\right\}$. Observe that $N_{G}(a) \subseteq N_{G}[\widetilde{a}]$. Since $\pi_{1} b=a b \notin E(G)$, we see that $\pi_{2} \cdots \pi_{n}$ is a $k$-thick Hamiltonian vertex ordering of $G-\pi_{1}$ and hence the induction assumption says that $G-\pi_{1}$ has a spanning $k$-rail between $\widetilde{a}$ and $b$. Applying Lemma 108 on $H=G-\pi_{1}$ yields the result that $G$ has a spanning $k$-rail between $a$ and $b$, as wanted.

b) $\Rightarrow \mathbf{h})$ Suppose that $\mathcal{I}$ is an interval representation of $G, a$ is a leftmost vertex and $b$ is a rightmost vertex with respect to $\mathcal{I}$. Further assume that $G$ has a spanning $k$-rail between $a$ and $b$ consisting of $k$ paths, say $P_{1}, \ldots, P_{k}$. For each $i \in[k-1]$, we can assume that the vertex adjacent to $b$ on $P_{i}$ is $b_{i} \notin\{a, b\}$. It is clear that $b, b_{1}, \ldots, b_{k-1}$ all fall in the rightmost clique with respect to $\mathcal{I}$ and so $P_{i}^{\prime}, i \in[k]$ give rise to a typical $k$-fan of $G$, where $P_{i}^{\prime}=P_{i}-b$ for $i \in[k-1]$ and $P_{k}^{\prime}=P_{k}$, as was to be shown. 
h) $\Rightarrow$ b) Let $\mathcal{I}$ be an interval representation of $G, a$ be a leftmost vertex and $U \in\left(\begin{array}{c}V(G) \backslash\{a\} \\ k\end{array}\right)$ be a subset of the rightmost clique of $G$ with respect to $\mathcal{I}$. Assume that we have a typical $k-(x, U)$-fan of $G$ consisting of $k$ paths $P_{1}, \ldots, P_{k}$ and we assume that $P_{k}$ contains a rightmost vertex $b \neq a$. We apply Lemma 104 on $P_{k}$ to get an $a, b$-path $P_{k}^{\prime}$ of $G$ satisfying $V\left(P_{k}^{\prime}\right)=V\left(P_{k}\right)$. For each $i \in[k-1]$, we assume that $P_{i}$ is an $a, b_{i}$-path and consider the path $P_{i}^{\prime}=P_{i} b_{i}\left(b_{i}, b\right)$. It is easy to check that $P_{1}^{\prime}, \ldots, P_{k}^{\prime}$ form the required typical $k$-rail of $G$ between $a$ and $b$, thus finishing the proof.

\subsubsection{Proofs of Theorems 23, 24, 27, 28, 29 and 30}

To prove Theorem 23 , we will need the following lemma, which, in some sense, is dual to Theorem 46. It is interesting that we will need this result again when proving Theorem 46.

Lemma 110 Let $k$ be a positive integer, let $G$ be an interval graph which is not $K_{k+1}$, and let $x$ be a simplicial vertex of $G$. If $G$ has a typical $k$-rail, then so does $G-x$.

Proof: Suppose that $\mathcal{I}$ is an interval representation of $G, a$ and $b$ are two vertices of $G$ such that $r_{\mathcal{I}}(a)=$ $\min \left\{r_{\mathcal{I}}(z): z \in V(G)\right\}$ and $\ell_{\mathcal{I}}(b)=\max \left\{\ell_{\mathcal{I}}(z): z \in V(G)\right\}$, and $\mathcal{T}$ is a spanning $k$-rail of $G$ between $a$ and $b$.

If $G$ is a complete graph, the result follows directly. So, we assume below that $G$ is not a complete graph and hence $a b \notin E(G)$. For any path

$$
P=\left(a=v_{1}, v_{2}, \ldots, v_{m}=b\right)
$$

of the given $k$-rail $\mathcal{T}$, as $a b \notin E(G)$, we will have $m \geq 3$.

CASE 1. $x \notin\{a, b\}$.

Assume that $x$ appears in a path $P=\left(a=v_{1}, v_{2}, \ldots, v_{m}=b\right)$ of the given $k$-rail $\mathcal{T}$, say $x=v_{i}$ for some $1<i<m$. We can replace the path $P$ in $\mathcal{T}$ by the path $\left(v_{1}, v_{2}, \ldots, v_{i-1}, v_{i+1}, v_{i+2}, \ldots, v_{m}\right)$ and then, as $a b \notin E(G)$, we obtain a required typical $k$-rail of $G-x$.

CASE 2. $x \in\{a, b\}$.

We may suppose $x=a$. Choose a vertex $a^{\prime} \in V(G) \backslash\{a\}$ such that $r_{\mathcal{I}}(a) \leq r_{\mathcal{I}}\left(a^{\prime}\right)=\min \left\{r_{\mathcal{I}}(z)\right.$ : $z \in V(G) \backslash\{a\}\}$. There is nothing to prove when $G-a$ is a complete graph. Accordingly, we may assume that $a^{\prime} \neq b$ and $a^{\prime} b \notin E(G)$. For any path $P$ in the given $k$-rail $\mathcal{T}$, say as displayed in Eq. 29], we can see that $a^{\prime} v_{2} \in E(G-x)$ and thus we can let $\widetilde{P}$ be the path $\left(a^{\prime}, v_{2}, \ldots, v_{m}\right)$ if $a^{\prime} \notin V(P)$, and let $\widetilde{P}$ be a spanning $a^{\prime}, b$-path for $G\left[v_{2}, \ldots, v_{m}\right]$ if $a^{\prime} \in V(P)$, as guaranteed by Lemma 104 Apparently, the set of paths $\{\widetilde{P}: P \in \mathcal{T}\}$ forms a typical $k$-rail of $G-x$.

Proof of Theorem 23: We first consider the backward direction. As $G\left[L_{s}\right]=G$ has a typical $k$-fan, we know from Theorem $22 \mathrm{~h}) \Rightarrow$ b) that $G$ has a typical $k$-rail.

Now, the backward direction. By Theorem $22 \mathrm{~b}) \Rightarrow \mathrm{h}$ ), it suffices to show that $G\left[L_{i}\right]$ has a typical $k$-rail for every $i \in[s]$. For $i=s, G\left[L_{i}\right]$ has a typical $k$-rail is merely our assumption. If $s>1, G[S]$ is not a complete graph for any $S \supsetneq L_{s-1}$ and $G\left[L_{s-1}\right]$ is obtained from $G\left[L_{s}\right]$ by chopping off all (simplicial) vertices from $C_{s} \backslash C_{s-1}$; Thus, Lemma 110 applies to say that $G\left[L_{s-1}\right]$ still has a typical $k$-rail. Using this argument repeatedly, we can conclude that $G\left[L_{i}\right]$ has a typical $k$-rail for every $i \in[s]$, as wanted. 
Let $P=\left(w_{0}, \ldots, w_{m}\right)$ be a path in a graph. For any two integers $i$ and $j$ such that $0 \leq i \leq j \leq$ $m$, we use the notation $\left[w_{i}, w_{j}\right]_{P}$ or $\left[w_{j}, w_{i}\right]_{P}$ for the path $\left(w_{i}, w_{i+1}, \ldots, w_{j}\right)$, we use the notation $\left[w_{i}, w_{j}\right)_{P}$ or $\left(w_{j}, w_{i}\right]_{P}$ for the path $\left(w_{i}, \ldots, w_{j-1}\right)$, we use the notation $\left(w_{i}, w_{j}\right]_{P}$ or $\left[w_{j}, w_{i}\right)_{P}$ for the path $\left(w_{i+1}, \ldots, w_{j}\right)$ and we use the notation $\left(w_{i}, w_{j}\right)_{P}$ or $\left(w_{j}, w_{i}\right)_{P}$ for the path $\left(w_{i+1}, \ldots, w_{j-1}\right)$.

Proof of Theorem 24: According to Theorem 21 a), we may assume that $\mathcal{H}(G)=k \geq 3$. We aim to show that $\mathcal{H}_{G}(u) \geq k-1$ holds for every $u \in V(G)$. Let $\mathcal{I}$ be an interval representation of $G$. Let $a$ and $b$ be an opposite pair of $G$ with respect to $\mathcal{I}$. We suppose that $a b \notin E(G)$ as otherwise $G$ is a complete graph and then everything is trivially true. It follows from g) $\Rightarrow$ e) in Theorem 22 that $\mathcal{H}_{G}(a)=\mathcal{H}_{G}(b)=k$. By g) $\Rightarrow$ a) in Theorem 22, there exists a spanning $k$-rail of $G$ between $a$ and $b$ which consists of $k$ paths, say $P_{1}, \ldots, P_{k}$. Therefore, without loss of generality, we assume that $u \in V\left(P_{1}\right) \backslash\{a, b\}$.

Let $S$ denote $V\left((a, u]_{P_{1}}\right)$. By the implication of $\left.\left.\mathrm{g}\right) \Rightarrow \mathrm{e}\right)$ in Theorem $22, G-S$ has an $\mathcal{H}(G-S)$-thick normal vertex ordering $\pi^{\prime}$ from $a$ to $b$. Let $\pi=[u, a]_{P_{1}} \underline{a} \pi^{\prime}$ and we will conclude the proof by showing that $\mathcal{H}_{G}(\pi) \geq k-1$.

First we check that $\mathcal{H}(G-S) \geq k-1$. In view of Theorem $22 \mathrm{~b}) \Rightarrow \mathrm{g}$ ), we merely need to find a spanning $(k-1)$-rail of $G-S$ between $a$ and $b$. But this is guaranteed by Lemma 105

Pick arbitrarily $x \in S$. By the definition of $a$ and $b$, Lemma 106 together with $a b \notin E(G)$ implies $N_{G}(x) \cap\left(V\left(P_{i}\right) \backslash\{a, b\}\right) \neq \emptyset$ for every $i \in[2, k]$. This gives $\left|N_{G}(x) \backslash S\right| \geq k-1$ and thus ends the proof.

Proof of Lemma 26: If $\mathcal{H}(G) \leq 2$, Theorem 21 a) implies $\mathcal{H}(G-v) \geq \mathcal{H}(G)-1$ for all $v \in V(G)$.

If $\mathcal{H}(G) \geq 2$, applying the $\mathrm{g}) \Rightarrow \mathrm{c}$ ) implication in Theorem 22 we know that $(G-v)-S$ is traceable for all $v \in V(G)$ and $S \in\left(\begin{array}{c}V(G-v) \\ \leq \mathcal{H}(G)-2\end{array}\right)$. By Theorem 22 c $) \Rightarrow \mathrm{g}$ ), this gives $\min _{v \in V(G)} \mathcal{H}(G-v) \geq \mathcal{H}(G)-1$.

Lemma 111 Let $G$ be an interval graph with at least two vertices and let $\min _{v \in V(G)} \mathcal{H}(G-v)=p$. If $p \geq 1$, then $\mathcal{H}(G) \geq p+1$.

Proof: Since $\mathcal{H}(G-v) \geq 1$ for all $v \in V(G)$ while interval graphs are all chordal, we deduce from Theorem 19 that $G$ is traceable. By g) $\Rightarrow$ c) in Theorem 22, from $\min _{v \in V(G)} \mathcal{H}(G-v)=p$ we can derive that $G-S$ is traceable for all nonempty set $S \in\left(\begin{array}{c}V(G) \\ \leq p\end{array}\right)$. Taken together, $G-S$ is traceable for all $S \in\left(\begin{array}{c}V(G) \\ \leq p\end{array}\right)$ and so the part of c) $\Rightarrow \mathrm{g}$ ) in Theorem 22 tells us that $\mathcal{H}(G) \geq p+1$, as wanted.

Proof of Theorem 27; Let $\min _{v \in V(G)} \mathcal{H}(G-v)=p$. It follows from Lemma 26 that

$$
\mathcal{H}(G) \leq p+1 .
$$

Since $G$ is traceable, Eq. (30) gives $p \geq 0$. If $p \geq 1$, Eq. (30) together with Lemma 111 entails Eq. (7). If $p=0$, Eq. (7) is guaranteed by Eq. (30) and the assumption of $\mathcal{H}(G) \geq 1$.

If $G$ is not a complete graph, we have $\mathcal{H}_{G}(v) \leq \mathcal{H}(G-v)$ for all $v \in V(G)$. In addition, Corollary 25 gives $\mathcal{H}(G)-1 \leq \mathcal{H}_{G}(v)$ for all $v \in V(G)$. This in conjunction with Eq. (7) implies Eq. [8), as desired. 
Proof of Theorem 28; For statement a), its backward direction comes from Lemma 111 and its forward direction follows from Lemma 26. Statement b) is immediate from Eq. (8) in Theorem 27

The subsequent two lemmas together demonstrates the equivalence between statement i) and statement iii) in Theorem 29 .

Lemma 112 Let $G$ be an interval graph and let $k$ be an integer such that $1<k<|V(G)|$. If $G$ is spanning $(k-1)$-fan-connected, then $G \in \digamma^{k}$.

Proof: Take $U \in\left(\begin{array}{c}V(G) \\ k-1\end{array}\right)$. Utilizing the implication of d) $\left.\Rightarrow \mathrm{g}\right)$ in Theorem 22 it is sufficient to illustrate that $G-U$ is traceable. Pick any interval representation $\mathcal{I}$ of $G$ and choose a leftmost vertex $a$ of $G-U$ with respect to $\mathcal{I}$. Because $G$ is spanning $(k-1)$-fan-connected, it has a spanning $(k-1)$ - $(a, U)$-fan. Thanks to Lemma 105 , the existence of this $(a, U)$-fan tells us that $G-U$ is traceable, as wanted.

Lemma 113 Let $k \geq 3$ be an integer. Every graph $G \in \digamma^{k}$ is spanning $(k-1)$-fan-connected.

Proof: We shall proceed by an induction on $k$.

If $k=3$, it is a consequence of Theorem 9 that $G$ is spanning 2-fan-connected.

We now turn to the case of $k>3$. Taking $x \in V(G)$ and $U \in\left(\begin{array}{c}V(G-x) \\ k-1\end{array}\right)$, we intend to find a spanning $(x, U)$-fan of $G$. Let $\mathcal{I}$ be an interval representation of $G$ and let $a$ and $b$ be an opposite pair of $G$ with respect to $\mathcal{I}$. By virtue of $\mathrm{g}$ ) implying a) in Theorem 22, there exists a spanning $k$-rail of $G$ between $a$ and $b$, say, consisting of $k$ distinct paths $P_{1}, \ldots, P_{k}$. As a result of $k-1>2$, we obtain $U \backslash\{a, b\} \neq \emptyset$ and so, without loss of generality, we may assume that $\left(V\left(P_{1}\right) \cap U\right) \backslash\{a, b\} \neq \emptyset$. By Lemma 106 $N_{G}[x] \cap V\left(P_{1}\right) \neq \emptyset$. Therefore, we can find a vertex $z \in N_{G}[x] \cap V\left(P_{1}\right)$ and a vertex $y \in\left(V\left(P_{1}\right) \cap U\right) \backslash\{a, b\}$ such that $V\left([z, y)_{P_{1}}\right) \cap U=\emptyset$. It follows from $x \notin U$ that $x \neq y$. Consequently, we may assume that either $x=z$ or $x \notin V\left([z, y]_{P_{1}}\right)$. Let

$$
S= \begin{cases}V\left([z, y]_{P_{1}}\right), & \text { if } x \notin V\left([z, y]_{P_{1}}\right) ; \\ V\left((z, y]_{P_{1}}\right), & \text { if } x=z .\end{cases}
$$

An application of Lemma 105 now tells us that $G-S$ has a typical $(k-1)$-rail and hence, taking into account Theorem 22 b) implying g), $G-S \in \digamma^{k-1}$. By the induction hypothesis, $G-S$ has a spanning $(k-2)-(x, U \backslash\{y\})$-fan, say $R$. Let

$$
P=\left\{\begin{aligned}
(x, z) \underline{z}[z, y]_{P_{1}}, & \text { if } x \neq z ; \\
{[z, y]_{P_{1}}, } & \text { if } x=z .
\end{aligned}\right.
$$

Adding $P$ into the $(k-2)$-fan $R$ yields a spanning $(k-1)-(x, U)$-fan of $G$, as was to be shown.

Proof of Theorem 29: The implication of iii) $\Rightarrow$ i) is guaranteed by Lemma 112 Taking $s=0$ and $s=k-3$ demonstrate ii) $\Rightarrow$ iii) and ii) $\Rightarrow$ iv), respectively. It remains to verify i) $\Rightarrow$ ii) and iv) $\Rightarrow$ i).

i) $\Rightarrow$ ii) Since $\digamma^{k} \supseteq \cdots \supseteq \digamma^{1}$, our task is to show that $G-S$ is spanning $(k-s-1)$-fan-connected. It follows from Corollary 25 that $G-S \in \digamma^{k-s}$ and so Lemma 113 gives the result. 
iv) $\Rightarrow$ i) Take any nonnegative integer $s \leq k-1$ and any $S \in\left({ }^{V(G)}\right)$. By the equivalence of c) and g) in Theorem 22, it suffices to show that $G-S$ is traceable. If $s \leq k-3$, then $G-S$ is Hamiltonian-connected and so is traceable. If $s \in\{k-2, k-1\}$, then we arbitrarily choose a $(k-3)$-subset $T$ of $S$. As $G-T$ is Hamiltonian-connected, $G-T$ contains a Hamiltonian path with the vertices from $S \backslash T$ as endpoints. This implies that $G-S$ is traceable, as asserted.

Proof of Theorem 30: As a consequence of Theorem 27, we can assume $k=t \geq 2$ and so what we really need to show is that $G \in \digamma^{k}$ if and only if $G$ is spanning $k$-rail-connected. The backward direction comes from the implication of $\mathrm{b}$ ) $\Rightarrow \mathrm{g}$ ) in Theorem 22. We subsequently turn attention to the forward direction.

Let $\mathcal{I}$ be an interval representation of $G$ and $a, b$ be two vertices of $G$ such that $r_{\mathcal{I}}(a)=\min \left\{r_{\mathcal{I}}(z)\right.$ : $z \in V(G)\}$ and $\ell_{\mathcal{I}}(b)=\max \left\{\ell_{\mathcal{I}}(z): z \in V(G)\right\}$. By the claim of $\left.\mathrm{g}\right) \Rightarrow$ a) in Theorem 22, we know that $G$ has a spanning $k$-rail between $a$ and $b$ in $G$. Letting $x$ and $y$ be any two vertices of $G$, our goal is to show the existence of a spanning $k$-rail of $G$ between $x$ and $y$.

It is clear that $G$ is spanning 2-rail-connected if and only if it is Hamiltonian if and only if it has a spanning 2-rail. So, the result for $k=2$ is straightforward.

We now try to prove the result for $k \geq 3$ under the assumption that the result is valid for $k-1$. If $G$ is a complete graph, then everything follows easily. Thus, let us assume that $a b \notin E(G)$ hereafter. We can also make the assumption that $\{a, b\} \neq\{x, y\}$. Suppose the spanning $k$-rail between $a$ and $b$ consists of $k$ distinct pairwise-internally-disjoint $a, b$-paths $P_{1}, \ldots, P_{k}$. Since $k \geq 3$, without loss of generality, we assume that $x, y \notin V\left(P_{k}\right) \backslash\{a, b\}$. By Lemma 106, there exist $u, v \in V\left(P_{k}\right) \backslash\{a, b\}$ such that $u x, v y \in E(G)$. Let $S=V\left([u, v]_{P_{k}}\right)$. It follows from Lemma 105 that $G-S$ has a spanning $(k-1)$-rail between $a$ and $b$ and so, by virtue of b) $\Rightarrow \mathrm{g}$ ) in Theorem 22, $G-S \in \digamma^{k-1}$. At this moment, we can apply the induction hypothesis to render a spanning $(k-1)$-rail of $G-S$ between $x$ and $y$. Adding the path $(x, u) \underline{u}[u, v]_{P_{k}} \underline{v}(v, y)$ into this $(k-1)$-rail, we obtain the required spanning $k$-rail of $G$ between $a$ and $b$.

\subsubsection{Proofs of Theorems 31 and 32}

The Hamiltonian thickness of a graph $G$ is the maximum height of its vertices. If we consider instead the minimum height, a trivial estimate is

$$
\min _{v \in V(G)} \mathcal{H}_{G}(v) \geq 2-|V(G)| .
$$

The next lemma provides a not so trivial estimate on the minimum height and can be thought of as a result about the fault-tolerance of the complete graph.

Lemma 114 Take two integers $n$ and $s$ such that $n \geq 2$ and $0 \leq s \leq\left(\begin{array}{l}n \\ 2\end{array}\right)$. Let $G$ be a graph with $n$ vertices and $\left(\begin{array}{l}n \\ 2\end{array}\right)-s$ edges. Then $\min _{u \in V(G)} \mathcal{H}_{G}(u) \geq n-s-2$ and there exists $\{u, w\} \in\left(\begin{array}{c}V(G) \\ 2\end{array}\right)$ such that $\min \left\{\mathcal{H}_{G}(u), \mathcal{H}_{G}(w)\right\} \geq n-s-1$.

Proof: We use induction on $n$. The claim is vacuously true when $n=2$. We now take $n>2$ and assume that the result holds for smaller $n$. Take $v \in V(G)$ so that $\operatorname{deg}_{G}(v)=\max _{w \in V(G)} \operatorname{deg}_{G}(w)$. Let $H=G-v$. 
CASE 1. $\operatorname{deg}_{G}(v)=n-1$.

Note that $H$ has $\left(\begin{array}{c}n-1 \\ 2\end{array}\right)-s$ edges. The induction assumption says that $\min _{u \in V(H)} \mathcal{H}_{H}(u) \geq n-s-3$ and there are at least two vertices $u$ and $w$ such that $\min \left\{\mathcal{H}_{H}(u), \mathcal{H}_{H}(w)\right\} \geq n-s-2$. For any $x \in$ $V(H)$, let $\pi$ be an ordering of $V(H)$ starting at $x$. If $\pi$ is Hamiltonian, $\mathcal{H}_{G}(\pi v)=\mathcal{H}_{H}(\pi)+1$; Otherwise, there exists $i \in[n-2]$ such that $\pi_{i} \pi_{i+1} \notin E(G)$ and it holds $\mathcal{H}_{G}\left(\pi_{1} \cdots \pi_{i} v \pi_{i+1} \cdots \pi_{n-1}\right)=\mathcal{H}_{H}(\pi)+1$. If $\tau$ is an $(n-s-2)$-thick Hamiltonian vertex ordering of $H$, it is clear that $v \tau$ is an $(n-s-2)$-thick Hamiltonian vertex ordering of $G$.

CASE 2. $\operatorname{deg}_{G}(v)=n-2$.

It is easy to see that $H$ cannot be a complete graph and so

$$
\mathcal{H}(H) \leq n-3
$$

holds.

If $n=3$, the graph $G$ has to be a graph with exactly one edge and the result is trivial. We now suppose $n \geq 4$. Note that $H$ has $\left(\begin{array}{c}n-1 \\ 2\end{array}\right)-(s-1)$ edges. By the induction assumption, $\min _{u \in V(H)} \mathcal{H}_{H}(u) \geq$ $n-s-2$ and we can get $\{u, w\} \in\left(\begin{array}{c}V(H) \\ 2\end{array}\right)$ such that $\min \left\{\mathcal{H}_{H}(u), \mathcal{H}_{H}(w)\right\} \geq n-s-1$.

Take $v^{\prime} \in N_{G}(v)$ and select an ordering $\pi=\pi_{1} \cdots \pi_{n-1}$ of $H$ such that $\pi_{1}=v^{\prime}$ and $\mathcal{H}_{H}(\pi)=$ $\mathcal{H}_{H}\left(v^{\prime}\right) \geq n-s-2$. It turns out that $\tau=v \pi_{1} \cdots \pi_{n-1}$ is an ordering of $G$ with $\mathcal{H}_{G}(\tau) \geq n-s-2$. For any ordering $\sigma=\sigma_{1} \cdots \sigma_{n-1}$ of $V(H)$, on account of Eq. (31) and our assumption that $n \geq 4$, it is easy to see that $\max \left\{\mathcal{H}_{G}(\widetilde{\sigma}), \mathcal{H}_{G}(\widehat{\sigma})\right\} \geq \mathcal{H}_{H}(\sigma)$, where $\widetilde{\sigma}=\sigma_{1} v \sigma_{2} \cdots \sigma_{n-1}$ and $\widehat{\sigma}=\sigma_{1} \cdots \sigma_{n-1} v$.

CASE 3. $\operatorname{deg}_{G}(v) \leq n-3$.

Note that $|E(H)|=\left(\begin{array}{c}n-1 \\ 2\end{array}\right)-\left(s-n+1+\operatorname{deg}_{G}(v)\right) \geq\left(\begin{array}{c}n-1 \\ 2\end{array}\right)-(s-2)$. The induction assumption says that $\min _{u \in V(H)} \mathcal{H}_{H}(u) \geq n-s-1$ and $H$ has at least two different vertices $u$ and $w$ such that $\min \left\{\mathcal{H}_{H}(u), \mathcal{H}_{H}(w)\right\} \geq n-s$. From $\max \left\{\operatorname{deg}_{G}(x): x \in V(G)\right\}=\operatorname{deg}_{G}(v) \leq n-3$ we deduce that $s \geq n$ and so $n-s-1 \leq-1$ and $n-s \leq 0$. For any ordering $\pi$ of $V(H)$ with $\mathcal{H}_{H}(\pi) \leq 0$, we can see that $\min \left\{\mathcal{H}_{G}(\pi v), \mathcal{H}_{G}(v \pi)\right\} \geq \mathcal{H}_{H}(\pi)-1$; For any ordering $\pi$ of $V(H)$ with $\mathcal{H}_{H}(\pi)>0$, it holds $\mathcal{H}_{G}(\pi v) \geq 0 \geq n-s-2$. Therefore, we can verify the result for $|V(G)|=n$ and complete the proof.

Lemma 115 Take two positive integers $k$ and $n$ such that $k<n$. Let $G$ be an n-vertex interval graph possessing a $k$-thick Hamiltonian vertex ordering $\pi$. Choose $\mathcal{E} \subseteq E(G)$ with $|\mathcal{E}|=s<k$ and let $G^{\prime}=G-\mathcal{E}$. Then $\min _{u \in V\left(G^{\prime}\right)} \mathcal{H}_{G^{\prime}}(u) \geq k-s-1$. Moreover, it holds $\mathcal{H}_{G^{\prime}}\left(\pi_{1}\right) \geq k-s$ when $n \geq k+2$.

Proof: If $n=k+1$, then $G$ is a complete graph and it is direct from Lemma 114 that $\min _{u \in V\left(G^{\prime}\right)} \mathcal{H}_{G^{\prime}}(u) \geq$ $n-s-2=k-s-1$.

We now assume $n \geq k+2$. By virtue of Theorem 24, it suffices to verify $\mathcal{H}_{G^{\prime}}\left(\pi_{1}\right) \geq k-s$. We do induction on $s$. When $s=0$, it holds $\mathcal{E}=\emptyset$ and so $\mathcal{H}_{G^{\prime}}\left(\pi_{1}\right)=\mathcal{H}_{G}\left(\pi_{1}\right) \geq k=k-s$, as desired. To proceed, suppose that $s \geq 1$ and that the result holds for smaller values of $s$. Let $i_{0}=\min \left\{i: \pi_{i} \in\right.$ $\partial(\mathcal{E}) \cup \Im(\mathcal{E})\}$.

CASE $1 . \quad i_{0} \geq n-k$.

The assumption of $n \geq k+2$ ensures the existence of $\pi_{n-k-1}$. Since $\mathcal{H}_{G}(\pi) \geq k$, we know that $G[\pi[n-k, n]]$ is a complete graph and $\left|N_{G}\left(\pi_{n-k-1}\right) \cap \pi[n-k, n]\right| \geq k$. By Lemma 114, there are at least two vertices $u$ and $w$ of $\pi[n-k, n]$ such that $\min \left\{\mathcal{H}_{G^{\prime}[\pi[n-k, n]]}(u), \mathcal{H}_{G^{\prime}[\pi[n-k, n]]}(w)\right\} \geq k+1-s-1=$ 
$k-s$. Without loss of generality, assume that $u \pi_{n-k-1} \in E(G)$ and $\tau$ is a $(k-s)$-thick Hamiltonian vertex ordering of $G^{\prime}[\pi[n-k, n]]$ with $\tau_{1}=u$. It follows from the minimality assumption on $i_{0}$ that $u \pi_{n-k-1} \notin \mathcal{E}$. In consequence, $\pi_{1} \cdots \pi_{n-k-1} u \tau_{2} \cdots \tau_{k+1}$ is a $(k-s)$-thick Hamiltonian vertex ordering of $G^{\prime}$ which starts at $\pi_{1}$.

CASE 2. $i_{0}<n-k$.

Note that $|\mathcal{E}|=s<k$ and $\pi_{i_{0}}$ has at least $k$ neighbors among $\pi\left[i_{0}+1, n\right]$. This gives the existence of $u \in \pi\left[i_{0}+1, n\right]$ so that $\pi_{i_{0}} u \in E(G) \backslash \mathcal{E}$.

Since $\pi_{i_{0}+1} \cdots \pi_{n}$ is a $k$-thick Hamiltonian vertex ordering of $G\left[\pi\left[i_{0}+1, n\right]\right]$, Corollary 25 dictates that $G\left[\pi\left[i_{0}+1, n\right]\right]$ has a $(k-1)$-thick Hamiltonian vertex ordering which starts at $u$. Recall that $i_{0}<n-k$ and so $n-i_{0} \geq(k-1)+2$. Notice additionally that $\left|\mathcal{E}^{\prime}\right| \leq s-1$, where $\mathcal{E}^{\prime}$ is the set of edges in $\mathcal{E}$ which are not incident to $\pi_{i_{0}}$. The induction assumption now implies that $\mathcal{H}_{G\left[\pi\left[i_{0}+1, n\right]\right]-\mathcal{E}^{\prime}}(u) \geq k-1-(s-1)=k-s$, which enables us obtain a $(k-s)$-thick Hamiltonian vertex ordering of $G\left[\pi\left[i_{0}+1, n\right]\right]-\mathcal{E}^{\prime}$ starting at $u$, say $\tau$. It is clear that $\pi_{1} \cdots \pi_{i_{0}} \tau_{1} \cdots \tau_{n-i_{0}}$ is a $(k-s)$-thick Hamiltonian vertex ordering of $G^{\prime}$ which starts at $\pi_{1}$, as desired.

Proof of Theorem 31: If $\mathcal{H}(G) \leq 2$, the result is a consequence of Theorem 21 b).

Suppose $\mathcal{H}(G)>2$. If $|\mathcal{E}|<\mathcal{H}(G)$, we can apply Lemmas 114 and 115 to get the conclusion. Otherwise, let $\mathcal{E}=\mathcal{E}_{1} \cup \mathcal{E}_{2}$ where $\left|\mathcal{E}_{1}\right|=\mathcal{H}(G)-1$ and $\left|\mathcal{E}_{2}\right|=|\mathcal{E}|-\mathcal{H}(G)+1$. By Lemmas 114 and 115 , the graph $G-\mathcal{E}_{1}$ contains a spanning subgraph $H$ with $\mathcal{H}(H)=\mathcal{H}(G)-(\mathcal{H}(G)-1)=1$. Making use of Theorem 21 b) again, the Hamiltonian thickness of $H-\left(\mathcal{E}_{2} \backslash E(H)\right)$ is at least $\mathcal{H}(H)-\left|\mathcal{E}_{2} \backslash E(H)\right|$. Finally, as $G-\mathcal{E}$ has $H-\left(\mathcal{E}_{2} \backslash E(H)\right)$ as a spanning subgraph, we obtain $\mathcal{H}(G-\mathcal{E}) \geq \mathcal{H}\left(H-\left(\mathcal{E}_{2} \backslash E(H)\right)\right) \geq$ $\mathcal{H}(H)-\left|\mathcal{E}_{2} \backslash E(H)\right| \geq \mathcal{H}(G)-|\mathcal{E}|$.

Proof of Theorem 32: The forward direction follows from the combination of Theorem 1. Corollary 25 and Theorem 31 The backward direction is guaranteed by Theorem 30 .

\subsubsection{Proofs of Theorems 33 and 35}

Lemma 116 For every interval graph $G$ and every positive integer $n$, it holds $\mathcal{H}\left(G \bigvee K_{n}\right)=\mathcal{H}(G)+n$.

Proof: Lemma 99 gives $\mathcal{H}\left(G \bigvee K_{n}\right) \geq \mathcal{H}(G)+n$. Corollary 25 implies $\mathcal{H}(G) \geq \mathcal{H}\left(G \bigvee K_{n}\right)-n$

Lemma 117 Let $G$ be a graph which is not a complete graph. For every $v \in V(G)$, it holds $\operatorname{sc}(G) \geq$ $\operatorname{sc}(G-v)-1$. If $v$ is a simplicial vertex of $G$, we even have $\operatorname{sc}(G) \geq \operatorname{sc}(G-v)$.

Proof: Let $G^{\prime}=G-v$.

We first assume that $G^{\prime}$ is a complete graph and hence $v$ must be simplicial. Let $\left|N_{G}(v)\right|=k$, where $0 \leq k \leq n-2$. It is not hard to check that $\operatorname{sc}(G)=2-k \geq 2-(n-2)=3-(n-1)=\operatorname{sc}\left(G^{\prime}\right)$.

Next consider the case that $G^{\prime}$ is not a complete graph. Take any scattering set $S^{\prime}$ of $G^{\prime}$. It follows that $\operatorname{sc}(G)+1 \geq \mathrm{c}\left(G-v-S^{\prime}\right)-\left|\{v\} \cup S^{\prime}\right|+1=\mathrm{c}\left(G^{\prime}-S^{\prime}\right)-\left|S^{\prime}\right|=\operatorname{sc}\left(G^{\prime}\right)$. In case that $v$ is simplicial in $G$, we have c $\left(G-S^{\prime}\right) \in\left\{\mathrm{c}\left(G^{\prime}-S^{\prime}\right), \mathrm{c}\left(G^{\prime}-S^{\prime}\right)+1\right\}$ and $\operatorname{sosc}(G) \geq \mathrm{c}\left(G-S^{\prime}\right)-\left|S^{\prime}\right| \geq$ $\mathrm{c}\left(G^{\prime}-S^{\prime}\right)-\left|S^{\prime}\right|=\operatorname{sc}\left(G^{\prime}\right)$. 
Proof of Theorem 33: By Theorem 15, it remains to show that the interval graph $G$ fulfils

$$
\operatorname{sc}(G)+\mathcal{H}(G) \geq 2 .
$$

Let $n=|V(G)|$ and let $\bar{n}$ be the number of vertices which is not universal in $G$. We finish the proof by an induction on $\bar{n}$.

When $\bar{n}=0$, namely when $G$ is a complete graph, we have $\mathcal{H}(G)=n-1$ and $\operatorname{sc}(G)=3-n$ and hence Eq. 32 holds. We now intend to verify Eq. 32 in the case that $\bar{n}>0$ under the assumption that Eq. (32) holds for smaller $\bar{n}$.

It follows from Lemmas 100 and 116 that $\operatorname{sc}(G)+\mathcal{H}(G)=\operatorname{sc}\left(G \bigvee K_{n}\right)+\mathcal{H}\left(G \bigvee K_{n}\right)$. Consequently, by adding enough number of universal vertices, Lemma 116 allows us to assume that

$$
\mathcal{H}(G) \geq 3 \text {. }
$$

By now, to prove Eq. (32) we need only consider the case that

$$
2-\operatorname{sc}(G)>3
$$

Let $\mathcal{I}$ be an interval representation of $G$, let $a$ be a leftmost vertex of $G$ with respect to $\mathcal{I}$, and let $\xi$ be the ordering of $V(G)$ according to the right-endpoint ordering of $\mathcal{I}$. Let $\pi$ be an output of $\operatorname{NP}(G, \xi, a)$, which, according to Eq. (33) and the implication of $\mathrm{g}) \Rightarrow \mathrm{e}$ ) in Theorem 22, must be a Hamiltonian vertex ordering of $G$. In particular, this gives

$$
\pi_{1} \pi_{2}, \pi_{2} \pi_{3} \in E(G)
$$

Since $G$ is not a complete graph and $\pi_{1}=a$ is a simplicial vertex of $G$, we see that $c\left(G-N_{G}\left(\pi_{1}\right)\right) \geq 2$. It then follows

$$
\left|N_{G}\left(\pi_{1}\right)\right|=\mathrm{c}\left(G-N_{G}\left(\pi_{1}\right)\right)-\left(\mathrm{c}\left(G-N_{G}\left(\pi_{1}\right)\right)-\left|N_{G}\left(\pi_{1}\right)\right|\right) \geq 2-\operatorname{sc}(G) .
$$

By Lemma $117 \operatorname{sc}(G) \geq \operatorname{sc}(G-a)$. Henceforth, the induction assumption says

$$
\mathcal{H}(G-a)=2-\operatorname{sc}(G-a) \geq 2-\operatorname{sc}(G) .
$$

To simplify the notation, let us write $G^{\prime}$ for $G-a=G-\pi_{1}$ and write $G^{\prime \prime}$ for $G-\left\{\pi_{1}, \pi_{2}\right\}$.

CASE 1. $r_{\mathcal{I}}\left(\pi_{2}\right)=\min \left\{r_{\mathcal{I}}\left(\pi_{i}\right): i \in[2, n]\right\}$ and so $\pi_{2} \cdots \pi_{n}$ is a normal vertex ordering of $G^{\prime}$.

By the equivalence of e) and g) in Theorem 22, we have $\mathcal{H}\left(G^{\prime}\right)=\mathcal{H}_{G^{\prime}}\left(\pi_{2} \cdots \pi_{n}\right)$. We can thus derive from Eqs. (34), 35), 36 and (37) that $\mathcal{H}_{G}(\pi) \geq 2-\operatorname{sc}(G)$, proving Eq. (32).

CASE 2. The graph $G^{\prime}$ is a complete graph.

We can surely modify the interval representation $\mathcal{I}$ a bit so that the condition in Case 1 holds and thus this case can be reduced to Case 1 .

CASE 3. $r_{\mathcal{I}}\left(\pi_{2}\right)>\min \left\{r_{\mathcal{I}}\left(\pi_{i}\right): i \in[3, n]\right\}$ and $G^{\prime}$ is not any complete graph.

In this case, we have $\pi_{1} \pi_{3} \notin E(G)$ and $r_{\mathcal{I}}\left(\pi_{3}\right)=\min \left\{r_{\mathcal{I}}\left(\pi_{i}\right): i \in[3, n]\right\}$. They justify the following sequence of reasoning:

- Since $\pi$ is a normal vertex ordering of $G, \mu=\pi_{3} \cdots \pi_{n}$ is a normal vertex ordering of $G^{\prime \prime}$; 
- Therefore, Theorem 22 implies $\mathcal{H}_{G^{\prime \prime}}(\mu)=\mathcal{H}\left(G^{\prime \prime}\right)$;

- It follows from Eq. (33) and Corollary 25 that $\mathcal{H}\left(G^{\prime \prime}\right) \geq \mathcal{H}(G)-2 \geq 1$;

- As $\pi_{1}$ is not universal in $G$, the induction assumption then yields

$$
1 \leq \mathcal{H}_{G^{\prime \prime}}(\mu)=\mathcal{H}\left(G^{\prime \prime}\right)=2-\operatorname{sc}\left(G^{\prime \prime}\right)
$$

- Noting that $\pi_{3} \in N_{G}\left(\pi_{2}\right) \backslash N_{G}\left(\pi_{1}\right)$ and that $\pi_{1}$ is the leftmost interval, we obtain $N_{G}\left(\pi_{1}\right) \subseteq$ $N_{G}\left(\pi_{3}\right) \subseteq N_{G}\left[\pi_{2}\right]$. This implies $\left|N_{G}\left(\pi_{2}\right) \cap \pi[3, n]\right| \geq\left|N_{G}\left(\pi_{1}\right) \cap \pi[2, n]\right|$, and hence, by Eq. [36,

$$
\left|N_{G}\left(\pi_{2}\right) \cap \pi[3, n]\right| \geq 2-\operatorname{sc}(G) .
$$

Combining Eqs. 35, 36, 38, and 39, once we can establish

$$
\operatorname{sc}\left(G^{\prime \prime}\right) \leq \operatorname{sc}(G)
$$

we will get $\mathcal{H}_{G}(\pi) \geq 2-\operatorname{sc}(G)$ and hence Eq. 32 will follow, as desired.

CASE 3.1. The graph $G^{\prime \prime}$ is a complete graph.

We observe that $\pi_{2}$ is simplicial in $G-\pi_{1}$ and $\pi_{1}$ is simplicial in $G$. Also, neither $G$ nor $G-\pi_{1}$ is a complete graph. Eq. (40) thus follows from Lemma 117.

CASE 3.2. The graph $G^{\prime \prime}$ is not a complete graph and so we can find a scattering set $S^{\prime \prime}$ of $G^{\prime \prime}$.

CASE 3.2.1. $N_{G}\left(\pi_{1}\right) \subseteq S^{\prime \prime} \cup\left\{\pi_{2}\right\}$.

This assumption tells us $\mathrm{c}\left(G-S^{\prime \prime}-\pi_{2}\right)=\mathrm{c}\left(G^{\prime \prime}-S^{\prime \prime}\right)+1 \geq 3$. Consequently, we get to $\operatorname{sc}\left(G^{\prime \prime}\right)=$ $\mathrm{c}\left(G^{\prime \prime}-S^{\prime \prime}\right)-\left|S^{\prime \prime}\right|=\mathrm{c}\left(G-S^{\prime \prime}-\pi_{2}\right)-1-\left|S^{\prime \prime}\right|=\mathrm{c}\left(G-S^{\prime \prime}-\pi_{2}\right)-\left|S^{\prime \prime} \cup\left\{\pi_{2}\right\}\right| \leq \operatorname{sc}(G)$, verifying Eq. 40.

CASE 3.2.2. $N_{G}\left(\pi_{1}\right) \backslash\left(S^{\prime \prime} \cup\left\{\pi_{2}\right\}\right) \neq \emptyset$.

Pick $u \in N_{G}\left(\pi_{1}\right) \backslash\left(S^{\prime \prime} \cup\left\{\pi_{2}\right\}\right)$. By the rule of the normal path algorithm, we know that

$$
\max \left\{\ell_{\mathcal{I}}(u), \ell_{\mathcal{I}}\left(\pi_{2}\right)\right\} \leq \min _{i \in[n]} r_{\mathcal{I}}\left(\pi_{i}\right)=r_{\mathcal{I}}\left(\pi_{1}\right)<r_{\mathcal{I}}\left(\pi_{3}\right)<r_{\mathcal{I}}\left(\pi_{2}\right) \leq r_{\mathcal{I}}(u) .
$$

This shows that

$$
N_{G}[u] \supseteq N_{G}\left[\pi_{2}\right] \supseteq N_{G}\left[\pi_{1}\right]
$$

and hence no two different connected components of $G^{\prime \prime}-S^{\prime \prime}$ can lie in the same connected component of $G-S^{\prime \prime}$ because of the adding of the two vertices $v_{1}$ and $v_{2}$. We thus conclude that

$$
\mathrm{c}\left(G^{\prime \prime}-S^{\prime \prime}\right) \leq \mathrm{c}\left(G-S^{\prime \prime}\right),
$$

from which we can derive

$$
\operatorname{sc}\left(G^{\prime \prime}\right)=\mathrm{c}\left(G^{\prime \prime}-S^{\prime \prime}\right)-\left|S^{\prime \prime}\right| \leq \mathrm{c}\left(G-S^{\prime \prime}\right)-\left|S^{\prime \prime}\right| \leq \operatorname{sc}(G)
$$

as asserted by Eq. (40). This ends the proof. 
Proof of Theorem 35: Both $\mathbf{d}) \Rightarrow \mathbf{e})$ and $\mathbf{e}) \Rightarrow \mathbf{a}$ ) are trivial. f) $\Leftrightarrow \mathbf{a}$ ) is implied by Theorem 33

a) $\Rightarrow$ b) This is due to Eq. (2) and $k \leq 0$.

b) $\Rightarrow$ c) Since the path cover number of $G$ is not greater than $2-k$, adding $1-k$ universal vertices will surely cause the existence of a Hamiltonian path.

c) $\Rightarrow$ d) By the equivalence of e) and g) in Theorem 22, all normal vertex orderings of $G \bigvee K_{1-k}$ are 1-thick Hamiltonian vertex orderings. This implies that all normal vertex orderings of $G$ are $k$-thick Hamiltonian vertex orderings.

\subsubsection{Proof of Theorem 36}

Lemma 118 Let $G$ be an interval graph and let $a^{\prime}$ and $b^{\prime}$ be an opposite pair of $G$. Let $k$ be a positive integer and assume that $G$ has a spanning $\left(\left\lfloor\frac{3 k}{2}\right\rfloor+1\right)$-rail between $a^{\prime}$ and $b^{\prime}$, say $\left\{P_{i}: i \in\left[\left\lfloor\frac{3 k}{2}\right\rfloor+1\right]\right\}$. Let $X=\left\{x_{i}: i \in[k]\right\} \in\left(\begin{array}{c}V(G) \backslash\left\{a^{\prime}, b^{\prime}\right\} \\ k\end{array}\right)$. Then $G$ has a path $P$ such that $x_{i}<_{P} x_{j}$ for every $i, j \in[k]$ with $i<j$ and that $G-V(P)$ has a Hamiltonian path from $a^{\prime}$ to $b^{\prime}$.

Proof: Without loss of generality, we assume that $n_{1} \geq \cdots \geq n_{\left\lfloor\frac{3 k}{2}\right\rfloor+1}$, where $n_{i}=\left|V\left(P_{i}\right) \bigcap X\right|$ for $i \in\left[\left\lfloor\frac{3 k}{2}\right\rfloor+1\right]$. Let $n_{0}=n_{1}+1$. Let $r$ and $s$ be the integers such that $n_{r}>1, n_{r+1} \leq 1, n_{r+s}>0$, $n_{r+s+1}=0$. Note that $k=|X|=\left|\cup_{i=1}^{r} V\left(P_{i}\right) \bigcap X\right|+\left|\cup_{i=1}^{s} V\left(P_{r+i}\right) \bigcap X\right|=\sum_{i=1}^{r} n_{i}+s \geq 2 r+s$. This then leads to

$$
\left\lfloor\frac{3 k}{2}\right\rfloor-r-s=\left\lfloor\frac{3 k}{2}\right\rfloor-r-\left(k-\sum_{i=1}^{r} n_{i}\right)=\left\lfloor\frac{k}{2}\right\rfloor-r+\sum_{i=1}^{r} n_{i} \geq \sum_{i=1}^{r} n_{i} .
$$

For any $i \in[k]$, we take $t_{i} \in\left[\left\lfloor\frac{3 k}{2}\right\rfloor+1\right]$ to be the number such that $x_{i} \in V\left(P_{t_{i}}\right)$. If $\left|V\left(P_{t_{i}}\right) \bigcap X\right|>1$, we choose the vertex $y_{i} \in\left(a^{\prime}, x_{i}\right]_{P_{t_{i}}}$ such that $\left[x_{i}, y_{i}\right]_{P_{t_{i}}} \cap X=\left\{x_{i}\right\}$ and $\left[x_{i}, y_{i}\right]_{P_{t_{i}}}$ contains as many as possible vertices, and we put $Q_{i}$ to be the $x_{i}, y_{i}$-path $\left[x_{i}, y_{i}\right]_{P_{t_{i}}}$, If $\left|V\left(P_{t_{i}}\right) \bigcap X\right|=1$, we let $x_{i}=y_{i}$ and let $Q_{i}$ be the path consisting of the vertex $x_{i}$ itself. We will construct the path $P$ in a greedy way and let it go through those paths $Q_{1}, \ldots, Q_{k}$ in this order and will walk from $x_{i}$ to $y_{i}$ when passing through $Q_{i}$.

To derive the path $P$, we will find a suitable connecting path $R_{i}$ in $G-\left\{a^{\prime}, b^{\prime}\right\}$ going from $y_{i}$ to $x_{i+1}$ for $i=1, \ldots, k-1$ one by one in this order. When $\left|V\left(P_{t_{i+1}}\right) \bigcap X\right|=1, R_{i}-y_{i}$ will be a subpath of $P_{t_{i+1}}$. When $\left|V\left(P_{t_{i+1}}\right) \cap X\right| \geq 2, R_{i}-\left\{y_{i}, x_{i+1}\right\}$ will be a subpath of $P_{t}$ for some $t \in\left\{r+s+1, \ldots,\left\lfloor\frac{3 k}{2}\right\rfloor\right\}$; Moreover, for every $t \in\left\{r+s+1, \ldots,\left\lfloor\frac{3 k}{2}\right\rfloor\right\}$, the path $P_{t}$ will intersect at most one such connecting path.

CASE 1. $\left|V\left(P_{t_{i+1}}\right) \cap X\right|=1$.

By Lemma 106, we can go from $y_{i}$ to one of its neighbors in $V\left(P_{t_{i+1}}\right) \backslash\left\{a^{\prime}, b^{\prime}\right\}$ and then walk along $P_{t_{i+1}}$ to $x_{i+1}$. In this way, we create a $y_{i}, x_{i+1}$-path $R_{i}$.

CASE 2. $\left|V\left(P_{t_{i+1}}\right) \bigcap X\right| \geq 2$.

By Eq. 441, we can find a $t \in\left\{r+s+1, \ldots,\left\lfloor\frac{3 k}{2}\right\rfloor\right\}$ such that $V\left(P_{t}\right) \cap\left(\cup_{s=1}^{i-1} V\left(R_{s}\right)\right)=\emptyset$. By Lemma 106 , we can find $\alpha_{i}, \beta_{i} \in V\left(P_{t}\right) \backslash\left\{a^{\prime}, b^{\prime}\right\}$ such that $\alpha_{i} y_{i}, \beta_{i} x_{i+1} \in E(G)$. The required $y_{i}, x_{i+1}$-path $R_{i}$ can now be formed by passing the edge $y_{i} \alpha_{i}$, then walking along $P_{t}$ from $\alpha_{i}$ to $\beta_{i}$ and finally using the edge $\beta_{i} x_{i+1}$ to get to the destination $x_{i+1}$. 
By examining the construction process of $P$, we see that $P_{\left\lfloor\frac{3 k}{2}\right\rfloor+1}$ has no common vertex with $P$ and for each $i \in\left[\left\lfloor\frac{3 k}{2}\right\rfloor\right]$, either $P_{i}$ has no common vertex with $P$ or $P_{i}\left[V\left(P_{i}\right) \backslash V(P)\right]$ consists of two subpaths of $P_{i}$, one with $a^{\prime}$ as an endpoint and the other with $b^{\prime}$ as an endpoint. We now apply Lemma 105 and find that $G-V(P)$ has a Hamiltonian path from $a^{\prime}$ to $b^{\prime}$, finishing the proof.

Proof of Theorem 36. Let $\mathcal{I}$ be an interval representation of $G$ and let $a$ and $b$ be two vertices of $G$ such that

$$
r_{\mathcal{I}}(a)=\min \left\{r_{\mathcal{I}}(z): z \in V(G)\right\} \text { and } \ell_{\mathcal{I}}(b)=\max \left\{\ell_{\mathcal{I}}(z): z \in V(G)\right\} .
$$

We introduce two new vertices $a^{\prime}$ and $b^{\prime}$ and let $G^{\prime}$ be the graph defined by

$$
V\left(G^{\prime}\right)=V(G) \cup\left\{a^{\prime}, b^{\prime}\right\}, E\left(G^{\prime}\right)=E(G) \cup\left\{a^{\prime} v: v \in N_{G}[a]\right\} \cup\left\{b^{\prime} v: v \in N_{G}[b]\right\},
$$

which has an interval representation $\mathcal{I}^{\prime}$ as specified by

$$
\mathcal{I}^{\prime}(v)=\left\{\begin{array}{l}
\mathcal{I}(v), \text { if } v \in V(G), \\
\mathcal{I}(a), \text { if } v=a^{\prime} \\
\mathcal{I}(b), \text { if } v=b^{\prime}
\end{array}\right.
$$

If $G$ is a complete graph, the two claims trivially hold. So, we assume below that $G$ is not a complete graph. To finish the proof, we take $X=\left\{x_{i}: i \in[k]\right\} \in\left(\begin{array}{c}V(G) \\ k\end{array}\right)$ and need to verify the following:

$\left.1^{\prime}\right)$ If $\mathcal{H}(G) \geq\left\lfloor\frac{3 k}{2}\right\rfloor+2$, then $G^{\prime}$ has a Hamiltonian path $P$ such that $x_{i}<_{P} x_{j}$ for all $i, j \in[k]$ with $i<j$;

$2^{\prime}$ ) If $\mathcal{H}(G) \geq\left\lfloor\frac{3 k}{2}\right\rfloor+3$, then $G^{\prime}$ has a Hamiltonian cycle $C$ such that $x_{1}, \ldots, x_{k}$ appear on $C$ in this ordering.

Indeed, noting that $a^{\prime} b^{\prime} \notin E\left(G^{\prime}\right)$ and both $a^{\prime}$ and $b^{\prime}$ are simplicial in $G^{\prime}$, we can simply remove $a^{\prime}$ and $b^{\prime}$ from the path/cycle of $G^{\prime}$ as asserted in $1^{\prime}$ ) or $2^{\prime}$ ) and then get the required path/cycle of $G$ as asserted in 1) or 2).

PROOF OF $1^{\prime}$ ): It is obvious that $\mathcal{H}\left(G^{\prime}\right) \geq \mathcal{H}(G) \geq\left\lfloor\frac{3 k}{2}\right\rfloor+2$. By Theorem $\left.\left.22 \mathrm{~g}\right) \Rightarrow \mathrm{a}\right), G^{\prime}$ possesses a typical $\left(\left\lfloor\frac{3 k}{2}\right\rfloor+2\right)$-rail $\left\{P_{i}: i \in\left[\left\lfloor\frac{3 k}{2}\right\rfloor+2\right]\right\}$ between $a^{\prime}$ and $b^{\prime}$. Suppose $V\left(P_{1}\right) \cap X=\emptyset$. Let $G^{\prime \prime}=G^{\prime}\left[V\left(G^{\prime}\right)-\left(V\left(P_{1}\right) \backslash\left\{a^{\prime}, b^{\prime}\right\}\right)\right]$. Then $G^{\prime \prime}$ has a typical spanning $\left(\left\lfloor\frac{3 k}{2}\right\rfloor+1\right)$-rail between $a^{\prime}$ and $b^{\prime}$. By Lemma 118 , there is a path $P^{\prime}$ of $G^{\prime \prime}$ such that $x_{i}<P^{\prime} x_{j}$ for all $i, j \in[k]$ satisfying $i<j$ and $G^{\prime \prime}-V\left(P^{\prime}\right)$ has a Hamiltonian path $Q^{\prime}$ from $a^{\prime}$ to $b^{\prime}$.

Pick an endpoint $u$ of $P^{\prime}$ and, by Lemma 106, we can choose $v \in V\left(Q^{\prime}\right)$ such that $v u \in E(G)$. Since $Q^{\prime}$ and $P_{1}$ are two paths from $a^{\prime}$ to $b^{\prime}, G^{\prime}\left[V\left(Q^{\prime}\right) \cup V\left(P_{1}\right)\right]$ has a Hamiltonian cycle and hence $G^{\prime}\left[V\left(Q^{\prime}\right) \cup V\left(P_{1}\right)\right]$ has a Hamiltonian path $Q^{\prime \prime}$ starting at $v$. It is now clear that the required path $P$ could be taken as $P^{\prime} \underline{u v} Q^{\prime \prime}$.

Proof of $\left.2^{\prime}\right)$ : Analogous to the proof of $1^{\prime}$ ), we have $\mathcal{H}\left(G^{\prime}\right) \geq \mathcal{H}(G) \geq\left\lfloor\frac{3 k}{2}\right\rfloor+3$ and so applying Theorem $22 \mathrm{~g}) \Rightarrow$ a) yields that $G^{\prime}$ possesses a typical $\left(\left\lfloor\frac{3 k}{2}\right\rfloor+3\right)$-rail $\left\{P_{i}: i \in\left[\left\lfloor\frac{3 k}{2}\right\rfloor+3\right]\right\}$ between $a^{\prime}$ and $b^{\prime}$. Suppose $V\left(P_{1}\right) \cap X=\emptyset$ and $V\left(P_{2}\right) \cap X=\emptyset$. Let $G^{\prime \prime}=G^{\prime}\left[V\left(G^{\prime}\right)-\left(V\left(P_{1}\right) \cup V\left(P_{2}\right) \backslash\left\{a^{\prime}, b^{\prime}\right\}\right)\right]$. Then $G^{\prime \prime}$ has a typical spanning $\left(\left\lfloor\frac{3 k}{2}\right\rfloor+1\right)$-rail between $a^{\prime}$ and $b^{\prime}$. By Lemma 118 , there is a path $P^{\prime}$ of 
$G^{\prime \prime}$, say $P^{\prime}=\left(w, p_{1}, \ldots, p_{s}, u\right)$, such that $x_{1}, \ldots, x_{k}$ appears on $P$ according to this ordering, and that $G^{\prime \prime}-V\left(P^{\prime}\right)$ has a Hamiltonian path $Q^{\prime}$ from $a^{\prime}$ to $b^{\prime}$. By Lemma 106, we can choose $w^{\prime} \in V\left(P_{1}\right) \backslash\left\{a^{\prime}, b^{\prime}\right\}$ and $u^{\prime} \in V\left(P_{2}\right) \backslash\left\{a^{\prime}, b^{\prime}\right\}$ so that $w w^{\prime} \in E(G)$ and $u u^{\prime} \in E(G)$.

Let $H=G^{\prime}\left[V\left(Q^{\prime}\right) \cup V\left(P_{1}\right) \cup V\left(P_{2}\right)\right]$. Note that both $V\left(P_{1}\right) \backslash\left\{a^{\prime}, b^{\prime}\right\}$ and $V\left(P_{2}\right) \backslash\left\{a^{\prime}, b^{\prime}\right\}$ are nonempty and so $Q^{\prime}, P_{1}$ and $P_{2}$ together form a typical 3-rail of $H$. Accordingly, we derive from Theorem 22 b) $\Rightarrow \mathrm{g}$ ) and Theorem 29 i) $\Rightarrow$ iv) that $H$ is Hamiltonian-connected. This means that we can find a Hamiltonian path $Q^{\prime \prime}$ of $H$ connecting $u^{\prime}$ and $w^{\prime}$, say $Q^{\prime \prime}=\left(u^{\prime}, q_{1}, \ldots, q_{t}, w^{\prime}\right)$. Now, setting $C=$ $\left\langle w, p_{1}, \ldots, p_{s}, u, u^{\prime}, q_{1}, \ldots, q_{t}, w^{\prime}\right\rangle$, we arrive at the required Hamiltonian cycle $C$, thereby finishing the proof.

\subsubsection{Proofs of Theorems 37, 40, 41, 42, and 43}

Proof of Theorem 37; i) If $G$ is not connected, we have $\operatorname{src}(G)=\operatorname{sfc}(G)=0$. If $G=K_{t}$ for $t \in[3]$, we have $\operatorname{src}(G)=\operatorname{sfc}(G)=t-1$. If $G$ is the path of length 2 , it holds $\operatorname{src}(G)=\operatorname{sfc}(G)=0$.

ii) By the equivalence between i) and iv) in Theorem 29, for the graph $G$ with at least 4 vertices, it holds $\mathcal{H}(G) \leq 2$ if and only if $G$ is not Hamiltonian-connected, which then amounts to saying that $\operatorname{src}(G)=\operatorname{sfc}(G)=0$.

iii) On account of $\mathcal{H}(G) \geq 3$, Theorem 29 i) $\Leftrightarrow$ iv) shows that $G$ is spanning 1-rail-connected while Theorem 30 implies that $G$ is spanning $q$-rail-connected for all $q \in\{2, \ldots, \mathcal{H}(G)\}$. Finally, the equivalence between a) and g) in Theorem 22 tells us that $G$ cannot be spanning $(\mathcal{H}(G)+1)$-rail-connected. This completes the proof of $\operatorname{src}(G)=\mathcal{H}(G)$.

It follows from Theorem 29 i) $\Rightarrow$ ii) that $\operatorname{sfc}(G) \geq \mathcal{H}(G)-1$. On the other hand, Theorem 29 iii) $\Rightarrow$ i) claims that $\operatorname{sfc}(G) \leq \mathcal{H}(G)-1$. Accordingly, we get to $\mathcal{H}(G)=\operatorname{sfc}(G)+1$.

Proof of Lemma 38: This follows from the equivalence of c) and g) in Theorem 22

Proof of Lemma 39: Li and Wu (73, Theorem 11) asserted that every connected rigid interval graph has a so-called consecutive ordering of its vertex set, which must correspond to a Hamiltonian path.

Proof of Theorem 40; Recall that every connected unit interval graph is a rigid interval graph; See Li and $\mathrm{Wu}(73)$, Panda and Das (89). Therefore, Lemma 39 tells us that unit interval graphs form a maroon class of interval graphs. The result is now a simple consequence of Lemma 38

Proof of Theorem 41; Matthews and Sumner (81, Theorem 10) showed that $\kappa(G)=2 \mathrm{t}(G)$ holds for every connected noncomplete $K_{1,3}$-free graph $G$ while Roberts (94) told us that every unit interval graph is $K_{1,3}$-free. Combining these with Eq. [10), Theorems 33 and 40 then conclude the proof. Note that we can avoid using Matthews and Sumner $\left(81\right.$, Theorem 10) by directly proving $2 \mathrm{t}(G)=\min _{C C^{\prime} \in E(T)}\left|C \cap C^{\prime}\right|$ where $T$ is a clique path of the connected noncomplete unit interval graph $G$.

Proof of Theorem 42; Let $G$ be a Hamiltonian-connected interval graph with $V(G)=[n]$. If $n=3$, then it is easy to check that $|E(G)|=3=3 n-6$. Suppose $n>3$. By the equivalence of i) and 
iv) in Theorem 29, $G$ must have a 3-thick Hamiltonian vertex ordering $\pi$. So, we obtain that $|E(G)|=$ $\sum_{i \in[n-1]} \mathrm{d}_{G, \pi}(i) \geq 3(n-3)+2+1=3 n-6$. It is obvious that for the equality to hold, $\pi$ must be an exact-3-thick Hamiltonian vertex ordering of $G$.

It remains to show that, for each integer $n \geq 3$, the graph with $n$ vertices as discussed above does exist. We define a unit interval graph $H$ with $|E(H)|=3 n-6$ by setting $V(H)=[n]$ and $N_{H}[i]=$ $[\max \{1, i-3\}, \min \{n, i+3\}]$ for each $i \in[n]$. We can check that either $H=K_{3}$ or $H \in \digamma^{3}$ and so, as a consequence of Theorem $9, H$ is Hamiltonian-connected, as required.

Proof of Theorem 43; Our task is to find a linear extension $\pi$ of $P$ such that $\mathcal{H}(G)=\mathcal{H}_{G}(\pi)$. Let $\mathcal{I}$ be an interval representation of $P$. We will check that the required $\pi$ could be any output of $\operatorname{NP}(G, \mathcal{I}, a)$ where $a$ is a vertex of $G$ such that $r_{\mathcal{I}}(a)=\min \left\{r_{\mathcal{I}}(v): v \in V(G)\right\}$.

The fact that $\mathcal{H}(G)=\mathcal{H}_{G}(\pi)$ follows from Theorem 22 e) $\Leftrightarrow \mathrm{g}$ ) when $\mathcal{H}(G) \geq 1$ and follows from Theorem 35 a) $\Leftrightarrow$ d) when $\mathcal{H}(G) \leq 0$. It is a consequence of Lemma 103 that $\pi$ is a linear extension of $P$.

\subsubsection{Proofs of Theorems 44,45 and 46}

Recall that we have defined $f_{G}(\pi)$ in Eq. (11) for any graph $G$ and any $\pi \in V(G)$ !.

Proof of Theorem 44: By Lemma 26, it holds $G-S \in \digamma^{\mathcal{H}(G)-|S|}$. It remains to show $\mathcal{H}(G-S) \leq$ $\mathcal{H}(G)-|S|$.

By the definition of normal vertex ordering, there is a normal vertex ordering $\pi^{\prime}$ of $G-S$ such that $\pi_{i}^{\prime}=\pi_{i}$ for every $i \in\left[f_{G}(\pi)\right]$. By the equivalence of e) and $\mathrm{g}$ ) in Theorem 22, we get

$$
\mathcal{H}(G-S)=\mathcal{H}_{G-S}\left(\pi^{\prime}\right)
$$

and

$$
\mathrm{d}_{G-S, \pi^{\prime}}(i)=\mathrm{d}_{G, \pi}(i)-|S|>\mathrm{d}_{G, \pi}\left(f_{G}(\pi)\right)-|S|=\mathcal{H}_{G}(\pi)-|S|=\mathcal{H}(G)-|S| \geq 0
$$

for $1 \leq i<f_{G}(\pi)$. This demonstrates $\mathcal{H}(G-S) \leq \mathcal{H}(G)-|S|$, as wanted.

Lemma 119 Let $G$ be an n-vertex interval graph with $\mathcal{H}(G)>0$ and let $\pi$ be a normal vertex ordering of $G$. Assume that $f_{G}(\pi)=t$. Then $\left\{\pi_{t+1}, \ldots, \pi_{n}\right\} \cap N_{G}\left(\pi_{t}\right)$ is a clique of $G$.

Proof: Suppose that $\pi$ is produced by the algorithm $\operatorname{NP}(G, \mathcal{I}, a)$, where $\mathcal{I}$ is an interval representation of $G$ and $r_{\mathcal{I}}(a)=\min \left\{r_{\mathcal{I}}(v): v \in V(G)\right\}$. By Theorem 22 $\left.\mathrm{f}\right) \Leftrightarrow \mathrm{g}$ ), we may suppose that $k=\mathcal{H}_{G}(\pi)=$ $\mathcal{H}(G)>0$. If $\left\{\pi_{t+1}, \ldots, \pi_{n}\right\} \cap N_{G}\left(\pi_{t}\right)$ were not a clique of $G$, then $t>1$ and there are two neighbors of $\pi_{t}$, say $\pi_{i}$ and $\pi_{j}$, such that $j>i>t$ and $\pi_{i} \pi_{j} \notin E(G)$. According to the rule of $\operatorname{NP}(G, \mathcal{I}, a)$, from $t>1$ we deduce that

$$
r_{\mathcal{I}}\left(\pi_{t}\right)=\min \left\{r_{\mathcal{I}}\left(\pi_{s}\right): t \leq s \leq n, \pi_{s} \pi_{t-1} \in E(G)\right\},
$$

and so we obtain from Lemma 103 that $N_{G}\left(\pi_{t-1}\right) \cap\left\{\pi_{t}, \ldots, \pi_{n}\right\} \subseteq N_{G}\left[\pi_{t}\right]$. As $\pi_{i} \pi_{j} \notin E(G)$ while $\pi_{j} \pi_{t} \in E(G)$, it follows from Lemma 103 that $r_{\mathcal{I}}\left(\pi_{i}\right)<\ell_{\mathcal{I}}\left(\pi_{j}\right)<r_{\mathcal{I}}\left(\pi_{t}\right)$ and hence Eq. (42) gives 
$\pi_{i} \in N_{G}\left(\pi_{t}\right) \backslash N_{G}\left(\pi_{t-1}\right)$. By now, we conclude that $\left|N_{G}\left(\pi_{t-1}\right) \cap \pi[t, n]\right| \leq\left|N_{G}\left(\pi_{t}\right) \cap \pi[t+1, n]\right|=k$, contradicting the fact that $f_{G}(\pi)=t$.

Proof of Theorem 45; i) $\Rightarrow$ ii) By Theorem $5, G$ is $(k-2)$-vertex-super-Hamiltonian and hence $(k-2)$ packable Hamiltonian.

ii) $\Rightarrow$ iii) $\Rightarrow$ iv) $\Rightarrow$ v) It is trivial.

v) $\Rightarrow$ i) Let $\mathcal{I}$ be an interval representation of $G$ and let $a$ be a vertex of $G$ such that $r_{\mathcal{I}}(a)=$ $\min \left\{r_{\mathcal{I}}(v): v \in V(G)\right\}$. Let $\pi$ be a Hamiltonian path constructed by the algorithm $\operatorname{NP}(G, \mathcal{I}, a)$. Taking $S=\emptyset$ in v) yields $\mathcal{H}(G)>0$. By Theorem $22 \mathrm{~g}) \Rightarrow \mathrm{e}), \mathcal{H}(G)=\mathcal{H}_{G}(\pi)$. Assume that $f_{G}(\pi)=t$. Then Lemma 119 asserts that $S=\left\{\pi_{t+1}, \ldots, \pi_{n}\right\} \cap N_{G}\left(\pi_{t}\right)$ is a clique of size $\mathcal{H}(G)$ in $G$. Applying Theorem 44 gives $\mathcal{H}(G-S)=0$ and so $G-S$ is not traceable. By the assumption of v), we get $\mathcal{H}(G)=|S| \geq k$, finishing the proof.

The equivalence among a), b), e) and f) in Theorem 22 paves the way for the following proof of Theorem 46

Proof of Theorem 46; Let $|V(G)|=n$. We shall perform induction on $n$. The base case of $n=2$ is trivial. We now assume $n>2$ and the result holds for smaller $n$.

Suppose that $\mathcal{I}$ is an interval representation of $G, a$ and $b$ are two vertices of $G$ such that $r_{\mathcal{I}}(a)=$ $\min \left\{r_{\mathcal{I}}(z): z \in V(G)\right\}$ and $\ell_{\mathcal{I}}(b)=\max \left\{\ell_{\mathcal{I}}(z): z \in V(G)\right\}$. Note that both $a$ and $b$ are simplicial vertices of $G$. We may assume hereafter that $G$ is not a complete graph (and so $a b \notin E(G)$ ) and $|S|<n-1$ as otherwise the result is obvious.

CASE 1. There exists a simplicial vertex $u$ of $G$ which does not fall into $S$.

By Lemma $110, G-u$ has a typical $k$-rail. We apply the induction assumption on the graph $G-u$ and the set $S \subsetneq V(G-u)$ and conclude the proof.

CASE 2. All simplicial vertices of $G$ are contained in $S$. In particular, this tells us $a, b \in S$.

By the implication of b) $\Rightarrow$ a) in Theorem 22, we know that there is a spanning $k$-rail between $a$ and $b$ in $G[S]$, say $\left\{P_{1}, \ldots, P_{k}\right\}$. It follows from $a b \notin E(G)$ that

$$
|S| \geq k+2 .
$$

CASE 2.1. $\quad N_{G}(a) \backslash S \neq \emptyset$ or $N_{G}(b) \backslash S \neq \emptyset$.

Without loss of generality, suppose $N_{G}(a) \backslash S \neq \emptyset$. Take any $x \in N_{G}(a) \backslash S$. It follows from $r_{\mathcal{I}}(a)=\min \left\{r_{\mathcal{I}}(z): z \in V(G)\right\}$ that $N_{G}[x] \supseteq N_{G}[a]$. Hence, assuming that $P_{1}=\left(a, q_{1}, q_{2}, \ldots, q_{s}, b\right)$, we know that $P_{1}^{\prime}=\left(a, x, q_{1}, q_{2}, \ldots, q_{s}, b\right)$ is a path from $a$ to $b$. Consequently, $\left\{P_{1}^{\prime}, P_{2}, \ldots, P_{k}\right\}$ is a typical $k$-rail of $G[S \cup\{x\}]$, completing the proof.

CASE 2.2. $N_{G}[a] \subseteq S$ and $N_{G}[b] \subseteq S$.

By the equivalence of b) and $\mathrm{f}$ ) in Theorem 22, our task is to find a vertex $x \in V(G) \backslash S$ and show the existence of a $k$-thick normal vertex ordering $\rho$ of $G[S \cup\{x\}]$.

Let $\pi$ be an output of $\operatorname{NP}(G, \mathcal{I}, a)$. By Theorem $22 \mathrm{~b}) \Rightarrow \mathrm{e})$, we have

$$
\mathcal{H}_{G}(\pi) \geq k
$$

and $\pi_{2} \in N_{G}\left[\pi_{1}\right]=N_{G}[a] \subseteq S$. 
CASE 2.2.1. $r_{\mathcal{I}}\left(\pi_{2}\right)=\min \left\{r_{\mathcal{I}}\left(\pi_{i}\right): i \in\{2, \ldots, n\}\right\}$.

By Lemma 110 , both $G-a$ and $G[S \backslash\{a\}]$ have typical $k$-rails. Using the induction hypothesis on $G-a$ and $S \backslash\{a\}$, we can find a vertex $x \in V(G-a) \backslash S$ such that $G\left[S^{\prime}\right]$ contains a typical $k$-rail where $S^{\prime}=(S \backslash\{a\}) \cup\{x\}$. We will be done if we can show that $G[S \cup\{x\}]=G\left[S^{\prime} \cup\{a\}\right]$ has a $k$-thick normal vertex ordering $\rho$.

Due to the equivalence of b) and e) in Theorem 22, we see that $G\left[S^{\prime}\right]$ has a $k$-thick normal vertex ordering $\rho^{\prime}$ starting from $\pi_{2}$. Owing to $N_{G}[a] \subseteq S$ as well as $\min \left\{\mathcal{H}_{G\left[S^{\prime}\right]}\left(\rho^{\prime}\right), \mathcal{H}_{G}(\pi)\right\} \geq k$, we find that $\rho=a \rho_{1}^{\prime} \cdots \rho_{\left|S^{\prime}\right|}^{\prime}$ will be a required $k$-thick normal vertex ordering of $G[S \cup\{x\}]$.

CASE 2.2.2. $r_{\mathcal{I}}\left(\pi_{2}\right)>\min \left\{r_{\mathcal{I}}\left(\pi_{i}\right): i \in\{2, \ldots, n\}\right\}$.

In this case, we can check that $r_{\mathcal{I}}\left(\pi_{3}\right)=\min \left\{r_{\mathcal{I}}\left(\pi_{i}\right): i \geq 2\right\}$ and $\pi_{1} \pi_{3} \notin E(G)$. This means that $\pi_{3}$ is the leftmost interval in $V\left(G-\pi_{1}\right)$ with respect to $\mathcal{I}, \pi_{3}$ is simplicial in $G$ and $\pi_{3} \in S$.

Let $\theta$ be an output of $\operatorname{NP}(G[S], \mathcal{I}, a)$. As $\left\{\pi_{1}, \pi_{2}, \pi_{3}\right\} \subseteq S$, we surely can require $\theta_{1}=\pi_{1}, \theta_{2}=\pi_{2}$ and $\theta_{3}=\pi_{3}$. From Theorem $\left.22 \mathrm{~b}\right) \Rightarrow \mathrm{e}$ ), we see that $\theta$ is a $k$-thick normal vertex ordering of $G[S]$. By Eq. (43), we have two cases to consider.

CASE 2.2.2.1. $|S|=k+2$.

Eq. (44) together with our assumption of $|S|<n-1$ tell us that $\mathrm{d}_{G, \pi}(3) \geq k$. Therefore, we can pick $x \in N_{G}\left(\pi_{3}\right) \backslash S$. Note that $(\{x\} \cup S) \backslash\left\{\pi_{1}\right\}$ is a clique in $G$. Accordingly, $\rho=\theta_{1} \cdots \theta_{k+2} x$ is a required $k$-thick normal vertex ordering of $G[S \cup\{x\}]$.

CASE 2.2.2.2. $|S| \geq k+3$.

In this case, both $G-\left\{\pi_{1}, \pi_{2}\right\}$ and $G\left[S \backslash\left\{\pi_{1}, \pi_{2}\right\}\right]$ have $k$-thick normal vertex orderings, which are obtained from $\pi$ and $\theta$ by deleting the first two terms there respectively. By virtue of the equivalence of b) and e) in Theorem 22, we can apply induction on $G-\left\{\pi_{1}, \pi_{2}\right\}$ and $G\left[S \backslash\left\{\pi_{1}, \pi_{2}\right\}\right]$ and get a vertex $x \in V(G) \backslash S$ such that $G[S \cup\{x\}]-\left\{\pi_{1}, \pi_{2}\right\}$ has a typical $k$-rail and hence, Theorem 22 b) $\Rightarrow$ e) applies, giving that $G[S \cup\{x\}]-\left\{\pi_{1}, \pi_{2}\right\}$ has a $k$-thick normal vertex ordering $\tau=\pi_{3} \tau_{2} \cdots \tau_{s}$ with respect to $\left.\mathcal{I}\right|_{(S \cup\{x\}) \backslash\left\{\pi_{1}, \pi_{2}\right\}}$, where $s=|S|-1$. To finish the proof, we let $\rho$ be the ordering $a \pi_{2} \tau_{1} \tau_{2} \cdots \tau_{s}=\pi_{1} \pi_{2} \tau_{1} \tau_{2} \cdots \tau_{s}=\pi_{1} \pi_{2} \pi_{3} \tau_{2} \cdots \tau_{s}$ and we intend to show that $\rho$ is a $k$-thick normal vertex ordering of $G[S \cup\{x\}]$.

Since $\pi$ and $\tau$ are normal vertex orderings with respect to $\mathcal{I}$ and $\left.\mathcal{I}\right|_{(S \cup\{x\}) \backslash\left\{\pi_{1}, \pi_{2}\right\}}$, respectively, what we really need to verify is $\mathcal{H}_{G}(\rho) \geq k$. This further reduces to checking the truth of the inequality

$$
\left|N_{G}\left(\rho_{i}\right) \cap\left\{\rho_{j}: s+2 \geq j \geq i+1\right\}\right| \geq k
$$

for $i \in\{1,2\}$, as we already know that $\tau$ is $k$-thick. Based on the fact that $\pi$ is $k$-thick, we can see that Eq. (45) holds for $i=1$ because $N_{G}\left[\pi_{1}\right] \subseteq S$, and Eq. (45] holds for $i=2$ because $N_{G}\left[\pi_{2}\right] \supseteq N_{G}\left[\pi_{1}\right] \cup\left\{\pi_{3}\right\}$ and $\pi_{1} \pi_{3} \notin E(G)$.

\subsubsection{Proofs of Theorems 52 and 53}

Proof of Theorem 52: By the rule of the normal path algorithm, the assumption of $\pi_{m-1} \pi_{m} \notin E(G)$ gives $\mathrm{d}_{G, \pi}(m-1)=0$ and so

$$
\pi_{m-1} \notin N_{G}[Q] .
$$

Consequently, according to Theorem $35 \mathrm{a}) \Leftrightarrow \mathrm{d}$ ), claim (II) directly follows from claim (I). 
We now try to prove (I). We use the shorthand $G^{\prime}$ for $G-N_{G}(Q)$. In view of the equivalence among Theorem 35 a), d) and e), we need to verify the following two points:

1) $\left\{\pi_{i-1} \pi_{i}, \pi_{i+1} \pi_{i}\right\} \subseteq E(G)$ and $\left\{\pi_{i-1}, \pi_{i+1}\right\} \cap N_{G}(Q)=\emptyset$ hold for every $\pi_{i} \in N_{G}(Q)$;

2) $\tau=\pi-N_{G}(Q)$ is a normal vertex ordering of $G^{\prime}$.

Suppose $\pi$ is an output of $\operatorname{NP}(G, \mathcal{I}, a)$ where $\mathcal{I}$ is an interval representation of $G$ and $a$ is a vertex of $G$ fulfilling $r_{\mathcal{I}}(a)=\min \left\{r_{\mathcal{I}}(v): v \in V(G)\right\}$. By Lemma 103 , we have

$$
\pi_{q} \in N_{G}(Q) \text { if and only if } r_{\mathcal{I}}\left(\pi_{q}\right) \geq \min \left\{\ell_{\mathcal{I}}\left(\pi_{j}\right): n \geq j \geq m\right\} \text { and } q \in[m-1] \text {; }
$$

Moreover, this combined with Lemma 103 again yields

$$
\pi_{p} \pi_{q} \in E(G)
$$

for those $p>q$ such that $\pi_{q} \in N_{G}(Q), \pi_{p} \notin N_{G}[Q]$.

To prove 1), it suffices to show for each $\pi_{i} \in N_{G}(Q)$ that $\left\{\pi_{i-1} \pi_{i}, \pi_{i+1} \pi_{i}\right\} \subseteq E(G)$ and that $\pi_{i+1} \notin$ $N_{G}(Q)$.

If $\pi_{i-1} \pi_{i} \notin E(G)$ or $i=1$, the rule of the normal path algorithm then asserts $r_{\mathcal{I}}\left(\pi_{i}\right)=\min \left\{r_{\mathcal{I}}\left(\pi_{j}\right)\right.$ : $n \geq j \geq i$. Noting that $\pi_{i} \in N_{G}(Q)$, it then follows from Eq. 47) that $\pi_{j} \in N_{G}(Q)$ for all $j \in$ $\{i, \ldots, m-1\}$, violating Eq. (46).

Since $\pi_{i} \in N_{G}(Q)$, Eq. (46) along with Eq. (48) implies

$$
\pi_{i} \pi_{m-1} \in E(G) \text {. }
$$

The rule of the normal path algorithm then shows that $\pi_{i+1} \pi_{i} \in E(G)$, as desired.

Finally, by Eqs. 46, 47) and (49), we get to

$$
r_{\mathcal{I}}\left(\pi_{i+1}\right) \leq r_{\mathcal{I}}\left(\pi_{m-1}\right)<\min \left\{\ell_{\mathcal{I}}\left(\pi_{j}\right): n \geq j \geq m\right\},
$$

and hence $\pi_{i+1} \notin N_{G}(Q)$ follows. This completes the proof of 1).

To prove 2), we will be done if we can check that $\tau$ is an output of $\operatorname{NP}\left(G^{\prime},\left.\mathcal{I}\right|_{V\left(G^{\prime}\right)}, a\right)$, that is, for each $i \in\left[\left|V\left(G^{\prime}\right)\right|-1\right], r_{\mathcal{I}}\left(\tau_{i+1}\right)=\min \left\{r_{\mathcal{I}}\left(\tau_{j}\right): \tau_{j} \in N_{G}\left(\tau_{i}\right) \cap \tau\left[i+1,\left|V\left(G^{\prime}\right)\right|\right]\right\}$ when $\mathrm{d}_{G^{\prime}, \tau}(i)>0$ and

$$
r_{\mathcal{I}}\left(\tau_{i+1}\right)=\min \left\{r_{\mathcal{I}}\left(\tau_{j}\right): j \in\left[i+1,\left|V\left(G^{\prime}\right)\right|\right]\right\}
$$

otherwise. We assume $\tau_{i}=\pi_{k}$. If $\tau_{i+1}=\pi_{k+1}$, then the claim is surely true. Consider the remaining possibility that $\tau_{i+1} \neq \pi_{k+1}$, namely

$$
\pi_{k+1} \in N_{G}(Q) .
$$

From Eqs. 47), 51] and the rule of the normal path algorithm, we find that

$$
N_{G}\left(\pi_{k}\right) \cap \pi[k+1, m] \subseteq N_{G}(Q),
$$

which implies $\mathrm{d}_{G^{\prime}, \tau}(i)=0$ and hence our goal is to establish Eq. 50.

Thanks to Eq. (51), applying 1) for $i=k+1$ yields

$$
\pi_{k+1} \pi_{k+2} \in E(G),
$$


as well as $\pi_{k+2} \notin N_{G}(Q)$ and so

$$
\pi_{k+2}=\tau_{i+1} .
$$

On the other hand, Eqs. (48) and (51) says that

$$
\pi[k+2, m] \backslash N_{G}(Q) \subseteq N_{G}\left(\pi_{k+1}\right) .
$$

Then,

$$
\begin{array}{rlrl}
r_{\mathcal{I}}\left(\tau_{i+1}\right) & =r_{\mathcal{I}}\left(\pi_{k+2}\right) \quad \text { (By Eq. [53]) } & \\
& =\min \left\{r_{\mathcal{I}}\left(\pi_{q}\right): q \in[k+2, n], \pi_{q} \pi_{k+1} \in E(G)\right\} & \text { (By Eq. (52)) } \\
& =\min \left\{r_{\mathcal{I}}\left(\pi_{q}\right): q \in[k+2, n], \pi_{q} \notin N_{G}(Q)\right\} & \text { (By Eqs. [47] and (54)) } \\
& =\min \left\{r_{\mathcal{I}}\left(\tau_{q}\right): q \in\left[i+1,\left|V\left(G^{\prime}\right)\right|\right]\right\} .
\end{array}
$$

This proves Eq. (50) and thus we are finished.

Lemma 120 Let $G$ be an interval graph with $n \geq 2$ vertices and let $\pi$ be a normal vertex ordering of $G$. Assume that $m=\max \left\{i \in\{2, \ldots, n\}: \pi_{i-1} \pi_{i} \notin E(G)\right\}$. Let $Q=\left\{\pi_{m}, \ldots, \pi_{n}\right\}$. If $N_{G}(Q) \neq \emptyset$ or $\mathcal{H}(G)<0$, then $G-N_{G}[Q]$ is a graph which is not a complete graph.

Proof: Let $\mathcal{I}$ be an interval representation of $G$ such that $\pi$ is a normal vertex ordering of $G$ with respect to $\mathcal{I}$. We distinguish two cases.

CASE $1 . \quad N_{G}(Q) \neq \emptyset$.

Take the minimum number $s$ such that $\pi_{s} \in N_{G}(Q)$. Note that Theorem 52 (II) implies $\pi_{m-1} \in$ $V(G) \backslash N_{G}[Q]$ and so $s<m-1$. By Eq. [47], $r_{\mathcal{I}}\left(\pi_{m-1}\right)<r_{\mathcal{I}}\left(\pi_{s}\right)$. According to the rule of the normal path algorithm, we now see that $s>1, \pi_{s-1} \pi_{s} \in E(G)$ and $\pi_{s-1} \pi_{m-1} \notin E(G)$. This gives $\left\{\pi_{s-1}, \pi_{m-1}\right\} \in\left(\begin{array}{c}V\left(G-N_{G}[Q]\right) \\ 2\end{array}\right)$ but $\pi_{s-1} \pi_{m-1} \notin E\left(G-N_{G}[Q]\right)$, implying that $G-N_{G}[Q]$ is not a complete graph.

CAse 2. $\mathcal{H}(G)<0$.

By Theorem 35 a) $\Leftrightarrow \mathrm{d}$ ), there exists $\ell \in[m-2]$ such that $\pi_{\ell} \pi_{\ell+1} \notin E(G)$. In the same way as we get Eq. (47), we can utilize Lemma 103 to derive that $\pi_{\ell}$ is to the left of $\pi_{m-1}$ and both of them are to the left of $Q$ with respect to the interval representation $\mathcal{I}$. This shows that $G-N_{G}[Q]$ is not a complete graph, as it contains both $\pi_{\ell}$ and $\pi_{m-1}$ but not has $\pi_{\ell} \pi_{m-1}$ as an edge.

Proof of Theorem 53: We induct on $n=|V(G)|$. If $n \leq 2$, the verification of the claim is straightforward. Suppose now $n>2$ and that the claim holds when $n$ is smaller. According to Theorem 22 e) $\Leftrightarrow \mathrm{g}$ ) and Theorem 35 a) $\Leftrightarrow d$ ), it holds

$$
\mathcal{H}(G)=\mathcal{H}_{G}(\pi) .
$$

To proceed, we will separate cases according to whether or not $\mathcal{H}(G)>0$ holds. 
CASE 1. $\mathcal{H}(G)=\mathcal{H}_{G}(\pi)>0$.

Let $S^{\prime}=\left\{\pi_{j}: \pi_{j} \in N_{G}\left(\pi_{f_{G}(\pi)}\right), j>f_{G}(\pi)\right\}$. From Theorem 44 and Eq. 55 we derive

$$
\mathcal{H}\left(G-S^{\prime}\right)=\mathcal{H}(G)-\left|S^{\prime}\right|=0 .
$$

Since $G$ is not a complete graph, $G-S^{\prime}$ cannot be a one vertex graph and so Eq. 56 means that $G-S^{\prime}$ is not any complete graph. Let $\tau$ be an output of $\mathrm{NP}\left(G-S^{\prime},\left.\mathcal{I}\right|_{V(G) \backslash S^{\prime}}, a\right)$ where $a$ is a vertex of $G-S^{\prime}$ satisfying $r_{\mathcal{I}}(a)=\min \left\{r_{\mathcal{I}}(v): v \in V\left(G-S^{\prime}\right)\right\}$. By induction hypothesis, the output of $S C^{\prime}\left(G-S^{\prime}, \tau, 0\right)$, say $S^{\prime \prime}$, is a scattering set of $G-S^{\prime}$. Using Theorem 33 and Eq. (56, we have $\mathrm{c}\left(G-S^{\prime}-S^{\prime \prime}\right)-\left|S^{\prime} \cup S^{\prime \prime}\right|=\left(\mathrm{c}\left(G-S^{\prime}-S^{\prime \prime}\right)-\left|S^{\prime \prime}\right|\right)-\left|S^{\prime}\right|=\operatorname{sc}\left(G-S^{\prime}\right)-\left|S^{\prime}\right|=2-\mathcal{H}\left(G-S^{\prime}\right)-\left|S^{\prime}\right|=$ $2-\mathcal{H}(G)=\operatorname{sc}(G)$. This proves that $S^{\prime} \cup S^{\prime \prime}$ is a scattering set of $G$, as desired.

CASE 2. $\mathcal{H}(G)=\mathcal{H}_{G}(\pi) \leq 0$.

Let $Q=\left\{\pi_{m}, \ldots, \pi_{n}\right\}$ where $m=\max \left\{i: 2 \leq i \leq n, \pi_{i-1} \pi_{i} \notin E(G)\right\}$. Note that $G$ is not any complete graph and so it has at least two vertices and so the existence of the number $m$ is guaranteed.

CASE 2.1. $\quad N_{G}(Q)=\emptyset$ and $\mathcal{H}_{G}(\pi)=0$.

Theorem 33 and Eq. (55) tell us that $\operatorname{sc}(G)=2-\mathcal{H}(G)=2-\mathcal{H}_{G}(\pi)=2$ and hence $\emptyset$ is a scattering set of $G$.

CASE 2.2. $N_{G}(Q) \neq \emptyset$ or $\mathcal{H}_{G}(\pi)<0$.

Since $N_{G}(Q) \neq \emptyset$ or $\mathcal{H}_{G}(\pi)<0$, it follows that

$$
\mathcal{H}(G)-\left|N_{G}(Q)\right|+1 \leq 0
$$

By Theorem 52 (II) and Eq. [57), we find that $\mathcal{H}\left(G-N_{G}[Q]\right)=\mathcal{H}(G)-\left|N_{G}(Q)\right|+1 \leq 0$ and $\pi-N_{G}[Q]$ is a normal vertex ordering of $G-N_{G}[Q]$. Surely, we then know from Theorem 35 a) $\Leftrightarrow$ d) that

$$
\mathcal{H}_{G-N_{G}[Q]}\left(\pi-N_{G}[Q]\right)=\mathcal{H}(G)-\left|N_{G}(Q)\right|+1 \leq 0 .
$$

As a consequence of Lemma $120, G-N_{G}[Q]$ is not any complete graph. By our induction hypothesis, the output of $S C^{\prime}\left(G-N_{G}[Q], \pi-N_{G}[Q], \mathcal{H}(G)-\left|N_{G}(Q)\right|+1\right)$, say $S$, is a scattering set of $G-N_{G}[Q]$. This means that

$$
\mathrm{c}\left(G-N_{G}[Q]-S\right)-|S|=\operatorname{sc}\left(G-N_{G}[Q]\right) .
$$

Accordingly, we arrive at

$$
\begin{array}{rlr}
\operatorname{sc}(G) & =2-\mathcal{H}(G) \quad \text { (By Theorem } 33 \\
& =2-\left(\mathcal{H}(G)-\left|N_{G}(Q)\right|+1\right)-\left|N_{G}(Q)\right|+1 \\
& =2-\mathcal{H}\left(G-N_{G}[Q]\right)-\left|N_{G}(Q)\right|+1 \quad \text { (By Theorem 52 (II)) } \\
& =\operatorname{sc}\left(G-N_{G}[Q]\right)-\left|N_{G}(Q)\right|+1 \quad \text { (By Theorem 33) } \\
& =\mathrm{c}\left(G-N_{G}[Q]-S\right)-|S|-\left|N_{G}(Q)\right|+1 \quad \text { (By Eq. 58) } \\
& =\mathrm{c}\left(G-S-N_{G}(Q)\right)-\left|S \cup N_{G}(Q)\right| . \quad\left(Q \text { is a component of } G-S-N_{G}(Q)\right)
\end{array}
$$

This proves that $S \cup N_{G}(Q)$ is a scattering set of $G$, finishing the proof. 


\subsubsection{Another proof of Theorem 33}

Having established Theorems 44 and 52 in last two subsections, we are ready to supply a new proof of Theorem 33. It is of some interest as it may suggest new approach in understanding the relationship between $\mathcal{H}(G)$ and $\operatorname{sc}(G)$ in more general contexts $(\$ 3.1 .2)$. We should point out that the proof of Theorem 52 involves the equivalence of a), d) and e) in Theorem 35 while our proof for that part of Theorem 35 is not relying on Theorem 33 namely no circular argument will be involved in the following proof.

Another proof of Theorem 33: By Theorem 15, we have $\operatorname{sc}(G)+\mathcal{H}(G) \leq 2$ and so our task is to show $\operatorname{sc}(G)+\mathcal{H}(G) \geq 2$. We shall proceed by induction on $|V(G)|$. If $|V(G)| \leq 2$, the result is easy to check. We now suppose $|V(G)|>2$ and divide the remaining proof into two cases.

CASE 1. $\mathcal{H}(G)>0$.

If $G$ is a complete graph, we have $\operatorname{sc}(G)=3-|V(G)|$ and $\mathcal{H}(G)=|V(G)|-1$ and so the result follows. We assume hereafter that $G$ is not a complete graph.

Let $T=\left\{\pi_{j}: \pi_{j} \in N_{G}\left(\pi_{f_{G}(\pi)}\right), j>f_{G}(\pi)\right\} \in\left(\begin{array}{c}V(G) \\ \mathcal{H}(G)\end{array}\right)$. By Theorem $44 . \mathcal{H}(G-T)=\mathcal{H}(G)-|T|=$ 0 . The induction hypothesis then gives

$$
\operatorname{sc}(G-T)=2-\mathcal{H}(G-T)=2 .
$$

Since $\mathcal{H}\left(K_{n}\right)=n-1$ and $G \neq K_{\mathcal{H}(G)+1}$, it also follow from $\mathcal{H}(G-T)=0$ that $G-T$ is not any complete graph. Take any scattering set of $G-T$, say $T^{\prime}$. We deduce from Eq. (59) that

$$
\operatorname{sc}(G) \geq \mathrm{c}\left(G-T-T^{\prime}\right)-\left|T \cup T^{\prime}\right|=\operatorname{sc}(G-T)-|T|=2-|T|=2-\mathcal{H}(G),
$$

as was to be shown.

CASE 2. $\mathcal{H}(G) \leq 0$.

Take any normal vertex ordering of $G$, say $\tau$. According to Theorem 35 a) $\Rightarrow$ d) and Eq. (2), $\pi(G)=$ $2-\mathcal{H}(G) \geq 2$ and there exist $t_{0}+1=1 \leq t_{1}<t_{2}<\cdots<t_{\pi(G)}=n$ such that $\left(\tau_{1}, \ldots, \tau_{t_{1}}\right)$, $\left(\tau_{t_{1}+1}, \ldots, \tau_{t_{2}}\right), \ldots,\left(\tau_{t_{\pi(G)-1}+1}, \ldots, \tau_{t_{\pi(G)}}=\tau_{n}\right)$ are $\pi(G)$ paths of $G$. Let $Q=\left\{\tau_{t_{\pi(G)-1}+1}, \ldots, \tau_{t_{\pi(G)}}\right\}$.

CASE 2.1. $N_{G}(Q) \neq \emptyset$.

Let $H=G-N_{G}(Q)$. It follows from Theorem $52(\mathrm{I})$ that $\mathcal{H}(H)=\mathcal{H}(G)-\left|N_{G}(Q)\right|<0$ and so we can take a scattering set of $H$, say $S$. We proceed as follows:

$$
\begin{aligned}
2 & \left.=2-\mathcal{H}(H)-\left|N_{G}(Q)\right|+\mathcal{H}(G) \quad \text { (By Theorem 52 }(\mathrm{I})\right) \\
& =\operatorname{sc}(H)-\left|N_{G}(Q)\right|+\mathcal{H}(G) \quad \text { (By induction hypothesis) } \\
& =\mathrm{c}(H-S)-|S|-\left|N_{G}(Q)\right|+\mathcal{H}(G) \\
& =\mathrm{c}\left(G-\left(S \cup N_{G}(Q)\right)\right)-\left|S \cup N_{G}(Q)\right|+\mathcal{H}(G) \\
& \leq \operatorname{sc}(G)+\mathcal{H}(G) .
\end{aligned}
$$

This verifies what we want.

CASE 2.2. $G-Q$ is traceable.

Since $G$ is the disjoint union of two traceable graphs $G-Q$ and $G[Q]$, Example 20 a) asserts $\operatorname{sc}(G)+$ $\mathcal{H}(G)=2+0=2$. 
CASE 2.3. $N_{G}(Q)=\emptyset$ and $G-Q$ is not traceable.

We have both $\mathcal{H}(G) \leq 0$ and $\mathcal{H}(G-Q) \leq 0$ and thus Eq. (2) tells us that $\pi(G)=2-\mathcal{H}(G)$ and $\pi(G-Q)=2-\mathcal{H}(G-Q)$. As $G-Q$ is not a complete graph, we can take a scattering set $S$ of $G-Q$. By induction assumption, it holds $2-\mathcal{H}(G-Q)=\operatorname{sc}(G-Q)$. We now have $2-\mathcal{H}(G)=\pi(G) \leq$ $1+\pi(G-Q)=3-\mathcal{H}(G-Q)=\operatorname{sc}(G-Q)+1=\mathrm{c}(G-Q-S)-|S|+1=\mathrm{c}(G-S)-|S| \leq \operatorname{sc}(G)$, finishing the proof.

\subsubsection{Proofs of Theorems 54, 55, 56 and 57}

Proof of Theorem 54; Let $n$ denote the number of vertices in the graph $G$ which are not universal and we will proceed by induction on $n$. If $n=0, G$ is a complete graph and we find that $\mathcal{H}(G)=\kappa(G)=$ $|V(G)|-1$ and $\alpha(G)=1$ and so the result holds. We now assume that $n>0$ (and so $\alpha(G) \geq 2$ ) and the result holds when $n$ is smaller.

Let $\mathcal{I}$ be an interval representation of $G$ and let $a \in V(G)$ be a leftmost vertex with respect to $\mathcal{I}$. Let $\pi$ be a normal vertex ordering produced by the algorithm $\operatorname{NP}(G, \mathcal{I}, a)$.

If $G-a$ is a complete graph, then $\mathcal{H}(G)=\left|N_{G}(a)\right|=\kappa(G)$ and $\alpha(G)=2$. This surely ensures the truth of the claim, as wanted.

We next consider the case that $G-a$ is not any complete graph and hence $\alpha(G-a) \geq 2$. Take any positive integer $t$. It is easy to see that $\alpha\left(G \bigvee K_{t}\right)=\alpha(G)$ and $\kappa\left(G \bigvee K_{t}\right)=\kappa(G)+t$; Lemma 116 says that $\mathcal{H}\left(G \bigvee K_{t}\right)=\mathcal{H}(G)+t$. Therefore, to finish the proof, we may even assume that $\kappa(G)>0$ and $\kappa(G)-\alpha(G)+2>0$. From $\kappa(G)>0$ it follows

$$
\pi_{1} \pi_{2} \in E(G) .
$$

Since $a$ is a leftmost vertex, $G$ is not a complete graph, we see that $\kappa(G-a) \geq \kappa(G)$. Applying the induction hypothesis on $G-a$, we now get

$$
\mathcal{H}(G-a) \geq \kappa(G-a)-\alpha(G-a)+2 \geq \kappa(G)-\alpha(G-a)+2 .
$$

CASE 1. $r_{\mathcal{I}}\left(\pi_{2}\right)=\min \left\{r_{\mathcal{I}}(v): v \in V(G-a)\right\}$.

Observe that $\pi^{\prime}=\pi_{2} \cdots \pi_{n}$ is a normal vertex ordering of $G-a$. By the statement that $\mathrm{g}$ ) implies e) in Theorem 22. Eq. 618 means that $\pi^{\prime}$ is a $(\kappa(G)-\alpha(G-a)+2)$-thick Hamiltonian vertex ordering of $G-a$. Note that we have Eq. 60, $\left|N_{G}(a)\right| \geq \kappa(G)$, and $\alpha(G) \geq \alpha(G-a) \geq 2$. Accordingly, $\pi$ is a $(\kappa(G)-\alpha(G)+2)$-thick Hamiltonian vertex ordering of $G$, which implies $\mathcal{H}(G)=\mathcal{H}_{G}(\pi) \geq$ $\kappa(G)-\alpha(G)+2$, as desired.

CASE 2. $r_{\mathcal{I}}\left(\pi_{2}\right)>\min \left\{r_{\mathcal{I}}(v): v \in V(G-a)\right\}$.

We have $\pi_{1} \pi_{3} \notin E(G)$ and $r_{\mathcal{I}}\left(\pi_{3}\right)=\min \left\{r_{\mathcal{I}}(v): v \in V(G-a)\right\}$. We can find a maximum stable set of $G-a$ which contains $\pi_{3}$ and so we can add $a$ into it to form a stable set of $G$. This tells us $\alpha(G)=\alpha(G-a)+1$. Combined with Eq. [61], this leads to $\mathcal{H}(G-a) \geq \kappa(G)-\alpha(G)+3$. By Theorem 24. there is a $(\kappa(G)-\alpha(G)+2)$-thick Hamiltonian vertex ordering of $G-a$ which starts at $\pi_{2}$, say $\tau$. Because of $\left|N_{G}(a)\right| \geq \kappa(G)$ and Eq. [60), $a \pi_{2} \tau_{2} \cdots \tau_{n-1}=a \tau_{1} \tau_{2} \cdots \tau_{n-1}$ is a $(\kappa(G)-\alpha(G)+2)$-thick Hamiltonian vertex ordering of $G$ and so $\mathcal{H}(G) \geq \kappa(G)-\alpha(G)+2$, completing the proof. 
Let $\mathcal{I}$ be an interval representation of a graph $G$. For any $u, v \in V(G)$, we write $\mathcal{I}(x)<\mathcal{I}(y)$ if $r_{\mathcal{I}}(x)<\ell_{\mathcal{I}}(y)$. For any two subsets $M$ and $N$ of $V(G)$, we write $M<_{\mathcal{I}} N$ if $\mathcal{I}(x)<\mathcal{I}(y)$ for every $x \in M$ and every $y \in N$.

Proof of Theorem 55: When $G$ is a complete graph, the assumption leads to $\mathcal{H}(G)=|V(G)|-1=$ $\kappa(G) \geq k+\alpha(G)=k+1$.

We now assume that $G$ is not any complete graph. By virtue of Theorem 33 , our task is to prove $\operatorname{sc}(G) \leq-k$.

Let $S$ be a scattering set of $G$. That is, $\operatorname{sc}(G)=p-|S|$, where $p=\mathrm{c}(G-S) \geq 2$. Let $\mathcal{I}$ be an interval representation of $G$ and list the connected components of $G-S$ as $C_{1}, \ldots, C_{p}$ so that $C_{1}<_{\mathcal{I}} \cdots<_{\mathcal{I}} C_{p}$. For each $v \in S$, we denote the number $\max \left\{i: v \in N_{G}\left(C_{i}\right)\right\}$ by $r(v)$ and denote the number $\min \{i$ : $\left.v \in N_{G}\left(C_{i}\right)\right\}$ by $\ell(v)$. It is easy to see that $v \in N_{G}\left(C_{j}\right)$ if and only if $j \in[\ell(v), r(v)]$. Because $G$ is a connected graph, we can find $v_{1}, \ldots, v_{q} \in S$ such that $r\left(v_{1}\right)<\cdots<r\left(v_{q-1}\right)<r\left(v_{q}\right)=p, v_{1}$ is an element from $N_{G}\left(C_{1}\right)$ so that $r\left(v_{1}\right)$ is as large as possible, and for each $i \in[q-1], v_{i+1}$ is an element from $N_{G}\left(C_{r\left(v_{i}\right)}\right)$ so that $r\left(v_{i+1}\right)$ is as large as possible.

Denote the set $N_{G}\left(C_{1}\right) \cap N_{G}\left(C_{2}\right)$ by $S_{1}$. Denote the set $N_{G}\left(C_{r\left(v_{i}\right)}\right) \cap N_{G}\left(C_{r\left(v_{i}\right)+1}\right)$ by $S_{i+1}$ for every $i \in[q-1]$. We make the convention that $r\left(v_{0}\right)$ stand for the number 1 . For each $i \in[q]$, we have

$$
r\left(v_{i}\right)-r\left(v_{i-1}\right)+1+k \leq \alpha_{G}\left(v_{i}\right)+k \leq \kappa_{G}\left(v_{i}\right) \leq\left|S_{i}\right| .
$$

Since $S_{i} \cap S_{j}=\emptyset$ holds for every $\{i, j\} \in\left(\begin{array}{c}{[q]} \\ 2\end{array}\right)$, it follows from Eq. [62] that $|S| \geq \sum_{i=1}^{q}\left|S_{i}\right| \geq$ $\sum_{i=1}^{q}\left(r\left(v_{i}\right)-r\left(v_{i-1}\right)+1+k\right)=p-1+q+k q \geq p+k$. This then implies $\operatorname{sc}(G)=p-|S| \leq$ $p-(p+k)=-k$, finishing the proof.

Proof of Theorem 56: By the implication of c) $\Rightarrow \mathrm{g}$ ) in Theorem 22, we can find $S \in\left(\begin{array}{c}V(G) \\ \mathcal{H}(G)\end{array}\right)$ such that $G-S$ is not traceable. This means that $G-(S \backslash\{u, v\})$ cannot have a spanning $u, v$-path and so $\widehat{c}_{G}^{1}(u, v) \leq|S \backslash\{u, v\}| \leq \mathcal{H}(G)$, as claimed.

Proof of Theorem 57: Since we always have $0 \leq \min \left\{\widehat{p}_{G}(u, v), \widehat{c}_{G}^{1}(u, v)\right\}$, the result is trivial when $\widehat{c}_{G}^{1}(u, v)=0$.

If $\widehat{c}_{G}^{1}(u, v)=1$, then $G$ has a spanning $u, v$-path and so $\widehat{p}_{G}(u, v) \geq 1=\widehat{c}_{G}^{1}(u, v)$.

If $\widehat{c}_{G}^{1}(u, v)=2$, then Theorem 56 says $\mathcal{H}(G) \geq 2$ and hence Theorem 1 applies to give $\widehat{p}_{G}(u, v) \geq$ $2=\widehat{c}_{G}(u, v)$.

We suppose that $\widehat{c}_{G}^{1}(u, v) \geq 3$ and hence, by Theorem 56. $\mathcal{H}(G) \geq 3$. By now, Theorem 37 iii) dictates that $\mathcal{H}(G)=\operatorname{src}(G)$, from which we can derive $\mathcal{H}(G) \leq \widehat{p}_{G}(u, v)$. Applying Theorem 56 now yields Eq. [12], as was to be shown.

4.2.12 Proofs of Theorems 61, 62, 63, 64, 66, 68 and 70

Proof of Theorem[61: Suppose $|V(G)|=n$. Take $i \in[n-1]$ and our task is to show that $\operatorname{dist}_{G}\left(\pi_{i}, \pi_{i+1}\right) \leq 3$. If $\operatorname{dist}_{G}\left(\pi_{i}, \pi_{i+1}\right) \leq 3$ would not hold, we can find $j \in[n]$ such that

$$
\min \left\{\operatorname{dist}_{G}\left(\pi_{i}, \pi_{j}\right), \operatorname{dist}_{G}\left(\pi_{j}, \pi_{i+1}\right)\right\} \geq 2
$$


and

$$
\operatorname{dist}_{G}\left(\pi_{i}, \pi_{i+1}\right)=\operatorname{dist}_{G}\left(\pi_{i}, \pi_{j}\right)+\operatorname{dist}_{G}\left(\pi_{j}, \pi_{i+1}\right) .
$$

Note that $\pi_{j}$ and $\pi_{i+1}$ cannot be separated by $N_{G}\left[\pi_{i}\right]$, as this means that we can find $x \in N_{G}\left[\pi_{i}\right]$ such that

$$
\operatorname{dist}_{G}\left(\pi_{i+1}, \pi_{j}\right)=\operatorname{dist}_{G}\left(\pi_{i+1}, x\right)+\operatorname{dist}_{G}\left(x, \pi_{j}\right)
$$

and so

$$
\begin{array}{rlr}
\operatorname{dist}_{G}\left(\pi_{i}, \pi_{i+1}\right) & \leq \operatorname{dist}_{G}\left(\pi_{i}, x\right)+\operatorname{dist}_{G}\left(x, \pi_{i+1}\right) \\
& \leq 1+\operatorname{dist}_{G}\left(x, \pi_{i+1}\right) & \text { (By } \left.x \in N_{G}\left[\pi_{i}\right]\right) \\
& \leq 1+\operatorname{dist}_{G}\left(\pi_{j}, \pi_{i+1}\right) & \text { (By Eq. 65) } \\
& <2+\operatorname{dist}_{G}\left(\pi_{j}, \pi_{i+1}\right) & \\
& \leq \operatorname{dist}_{G}\left(\pi_{i}, \pi_{i+1}\right), & \text { (By Eqs. 63) and 64) }
\end{array}
$$

which is surely absurd. By symmetry, we also know that $\pi_{j}$ and $\pi_{i}$ cannot be separated by $N_{G}\left[\pi_{i+1}\right]$.

If $j<i$, by the definition of path ordering, either $\pi_{j} \pi_{i+1} \in E(G)$ or $\pi_{j}$ and $\pi_{i+1}$ are separated by $N_{G}\left[\pi_{i}\right]$; If $j>i+1$, by the definition of path ordering, either $\pi_{j} \pi_{i} \in E(G)$ or $\pi_{j}$ and $\pi_{i}$ are separated by $N_{G}\left[\pi_{i+1}\right]$. As we have seen above, none of these four possibilities can actually occur, reaching a desired contradiction.

Proof of Theorem 62, We assume that $k \geq 3$ and that $\pi$ is a path ordering of $G$. Let $|V(G)|=n$ and take $i \in[n-1]$. To complete the proof, we assume that

$$
\left\{\pi_{i+1}, \ldots, \pi_{n}\right\} \backslash N_{G^{k}}\left(\pi_{i}\right) \neq \emptyset
$$

and aim to show that $\mathrm{d}_{G^{k}, \pi}(i) \geq(k-2) p=p$ when $k=3$ and that $\mathrm{d}_{G^{k}, \pi}(i) \geq(k-1) p$ when $k \geq 4$.

For each positive integer $r$, let $W^{r}=\left\{\pi_{\ell}: n \geq \ell>i\right.$, $\left.\operatorname{dist}_{G}\left(\pi_{i}, \pi_{\ell}\right)=r\right\}$. By Eq. (66), there exists an integer $j$ such that $n \geq j>i$ and $\operatorname{dist}_{G}\left(\pi_{i}, \pi_{j}\right)>k$. Our plan is to show that $W^{2}, \ldots, W^{k-1}$ are all $\pi_{i}, \pi_{j}$-separators in $G$ when $k \geq 3$ and that $W^{k}$ is also a $\pi_{i}, \pi_{j}$-separator in $G$ when $k \geq 4$. As $G$ is $p$-connected, this will give

$$
\mathrm{d}_{G^{k}, \pi}(i) \geq \sum_{r=2}^{k}\left|W^{r}\right| \geq \begin{cases}(k-1) p, & \text { if } k \geq 4 \\ (k-2) p, & \text { if } k=3\end{cases}
$$

as desired.

Pick arbitrarily $r \in\{2, \ldots, \min \{k, 2 k-4\}\}$ and let $H=G-W^{r}$. Suppose, by way of contradiction, we can find a shortest $\pi_{i}, \pi_{j}$-path in $H$, say $P=\left(x_{0}, \ldots, x_{t}\right)$ where $\pi_{i}=x_{0}$ and $\pi_{j}=x_{t}$. Note that

$$
t=\operatorname{dist}_{H}\left(\pi_{i}, \pi_{j}\right) \geq \operatorname{dist}_{G}\left(\pi_{i}, \pi_{j}\right)>k \geq r .
$$

Pick $\ell \in[n]$ so that $\pi_{\ell}=x_{r}$ and so

$$
\operatorname{dist}_{H}\left(\pi_{i}, \pi_{\ell}\right)=r
$$

and

$$
\operatorname{dist}_{H}\left(\pi_{\ell}, \pi_{j}\right)=\operatorname{dist}_{H}\left(\pi_{i}, \pi_{j}\right)-r=t-r \in[(t-k)+(k-r), t-2] \subseteq[1+k-r, t-2] .
$$

For every $v \in W^{1} \cup \cdots \cup W^{r-1}$, it is clear that $\operatorname{dist}_{H}\left(\pi_{i}, v\right)=\operatorname{dist}_{G}\left(\pi_{i}, v\right)$. Accordingly, from Eq. 67) and $H=G-W^{r}$, we conclude that

$$
\ell<i
$$


CASE 1. $r<k$.

By Eq. (68), $\operatorname{dist}_{H}\left(\pi_{\ell}, \pi_{j}\right) \geq 2$ and so $\pi_{\ell} \pi_{j} \notin E(G)$. Considering the path ordering $\pi$ and the three indices $\ell<i<j$, we now find that $N_{G}\left[\pi_{i}\right]$ will intercept the $\pi_{\ell}, \pi_{j}$-path $\left(x_{r}, x_{r+1}, \ldots, x_{t}\right)$ in $G$ (which is also a $\pi_{\ell}, \pi_{j}$-path in $\left.H\right)$. This implies $t=\operatorname{dist}_{H}\left(\pi_{i}, \pi_{j}\right) \leq 1+\operatorname{dist}_{H}\left(\pi_{\ell}, \pi_{j}\right)$, violating Eq. 687.

CASE 2. $r=k$.

Since $r \leq 2 k-4$, we have $k \geq 4$ in this case. Let $m$ be the index such that $\pi_{m}=x_{k-2}$. Because $t \geq k+1>k-2 \geq 2$ and $P$ is a shortest $\pi_{i}, \pi_{j}$-path in $H$, we obtain

$$
N_{G}\left[\pi_{j}\right] \cap\left\{x_{k-1}, \ldots, x_{1}\right\}=\emptyset=N_{G}\left[\pi_{i}\right] \cap\left\{x_{k-2}, \ldots, x_{t}\right\} .
$$

CASE 2.1. $m<i$.

We have $m<i<j$ now and $\left(x_{k-2}, x_{k-1}, \ldots, x_{t}\right)$ is a $\pi_{m}, \pi_{j}$-path in $G$. But then Eq. (69) tells us that $\pi$ cannot be a path ordering of $G$, which is a required contradiction.

CASE 2.2. $m>i$.

At this moment, we have $m>i>\ell, \pi_{m} \pi_{\ell}=x_{k-2} x_{k} \notin E(G)$, and that, by Eq. [69), $N_{G}\left[\pi_{i}\right]$ cannot intercept the path $\left(\pi_{m}=x_{k-2}, x_{k-1}, x_{k}=\pi_{\ell}\right)$, contradicting the assumption that $\pi$ is a path ordering of $G$.

Lemma 121 Let $P$ be a poset and $G$ be its cocomparability graph. If $\pi=\pi_{1} \cdots \pi_{n}$ is a linear extension of $P$, then every connected component of $G$ consists of a set of consecutive elements in $\pi$.

Proof: Otherwise, there exist $i<j<k$ such that $\pi_{i} \pi_{k} \in E(G)$ and $\pi_{i} \pi_{j}, \pi_{j} \pi_{k} \notin E(G)$, which contradicts the transitivity property of a poset.

Recall the definition of an ordinary ordering as illustrated before Theorem 63

Lemma 122 Let $P$ be a poset. Let $G$ be the cocomparability graph and let $\pi=\pi_{1} \cdots \pi_{n}$ be an ordinary ordering of $P$. If $G$ is connected, then $G\left[\pi_{i}, \ldots, \pi_{n}\right]$ is connected for every $i \in[n]$.

Proof: We prove the claim by induction on $i$. When $i=1$, the claim is direct from the assumption. Suppose we already know that $G\left[\pi_{i}, \ldots, \pi_{n}\right]$ is connected and we intend to show that $G\left[\pi_{i+1}, \ldots, \pi_{n}\right]$ is connected provided $i \leq n-1$. If this were not true, by Lemma 121 , we may assume that $G\left[\pi_{i+1}, \ldots, \pi_{j}\right]$ and $G\left[\pi_{j+1}, \ldots, \pi_{k}\right]$ are two connected components of $G\left[\pi_{i+1}, \ldots, \pi_{n}\right]$, where $i<j<k$. Because $G\left[\pi_{i}, \ldots, \pi_{n}\right]$ is connected, there exists $\ell$ such that $j+1 \leq \ell \leq k$ and $\pi_{i} \pi_{\ell} \in E(G)$. Since $\pi$ is an ordinary ordering of the poset $P$, it is a linear extension of $P$. Thus, by the transitivity of a poset and the fact that $G$ is the cocomparability graph of $P$, we obtain from $N_{G}\left(\pi_{\ell}\right) \cap\left\{\pi_{i+1}, \ldots, \pi_{j}\right\}=\emptyset$ that $\left\{\pi_{i+1}, \ldots, \pi_{j}\right\} \subseteq N_{G}\left(\pi_{i}\right)$. It then follows

$$
\pi_{i+1} \uparrow_{P} \supseteq\left\{\pi_{j+1}, \ldots, \pi_{n}\right\} \supsetneq\left\{\pi_{j+1}, \ldots, \pi_{n}\right\} \backslash\left\{\pi_{\ell}\right\} \supseteq \pi_{i} \uparrow_{P} .
$$

This shows that $\pi_{i}$ cannot be a bottom element in the subposet of $P$ induced by $\left\{\pi_{i}, \ldots, \pi_{n}\right\}$. This contradiction validates our claim, as wanted.

Proof of Theorem 63: Assume $\pi=\pi_{1} \cdots \pi_{n}$. By Lemma 122, we only need to show that $\operatorname{dist}_{G}\left(\pi_{i}, \pi_{i+1}\right)$ $\leq 2$ for every $i \in[n-2]$. 
Take $i \in[n-1]$. It follows from Lemma 122 that $G\left[\pi_{i}, \ldots, \pi_{n}\right]$ is connected. Henceforth, there exists $j>i$ such that $\pi_{i} \pi_{j} \in E(G)$. If $\pi_{i} \pi_{i+1} \in E(G)$, then $\operatorname{dist}_{G}\left(\pi_{i}, \pi_{i+1}\right)=1$. If $\pi_{i} \pi_{i+1} \notin E(G)$, then, as $\pi_{i} \cdots \pi_{n}$ is a linear extension of the subposet induced by $\left\{\pi_{i}, \ldots, \pi_{n}\right\}$, we will get $\pi_{i+1} \pi_{j} \in E(G)$, showing that $\operatorname{dist}_{G}\left(\pi_{i}, \pi_{i+1}\right)=2$.

Proof of Theorem 64: Assume that $|V(G)|=n$. If $n \leq 2$, the result is trivial. We hence only consider the case of $n \geq 3$. Let $P$ be a poset such that $G$ is the cocomparability graph of $P$ and let $\pi$ be an ordinary ordering of $P$. By Theorem $63, \pi$ is a 2-good vertex ordering of $G$, which implies that $\pi$ is a Hamiltonian vertex ordering of $G^{k}$. It remains to prove that $\mathrm{d}_{G^{k}, \pi}(i) \geq \min \{(k-1) p, n-i\}$ for all $i \in[n]$.

Fix an arbitrary element $i$ from $[n-1]$ and we intend to show that either $N_{G^{k}}\left(\pi_{i}\right) \supseteq\left\{\pi_{i+1}, \ldots, \pi_{n}\right\}$ or $\mathrm{d}_{G^{k}, \pi}(i) \geq(k-1) p$. Let $W=\left\{\pi_{j}: j \in[i]\right\}$. For any nonnegative integer $r$, let $W^{r}=\{v \in V(G)$ : $\left.\min \left\{\operatorname{dist}_{G}(v, u): u \in W\right\}=r\right\}$. Let $t=\max \left\{r: W^{r} \neq \emptyset\right\}$.

If $\pi_{\ell} \in N_{G}(W) \backslash N_{G}\left(\pi_{i}\right)$, then $\ell>i$ and there exists $j \in[i-1]$ such that $\pi_{j} \pi_{\ell} \in E(G)$. Because $\pi$ is a linear extension of $P$, it holds $\pi_{i} \pi_{j} \in E(G)$. This demonstrates that $N_{G^{2}}\left(\pi_{i}\right) \supseteq N_{G}(W)=W^{1}$ and so, for each $s \in\{2, \ldots, t+1\}$,

$$
N_{G^{s}}\left(\pi_{i}\right) \supseteq W^{1} \cup \cdots \cup W^{s-1} .
$$

If $t+1 \leq k$, then it follows from Eq. (70) that $N_{G^{k}}\left(\pi_{i}\right) \supseteq W^{1} \cup \cdots \cup W^{t}=\left\{\pi_{i+1}, \ldots, \pi_{n}\right\}$. We now assume that $t \geq k$. For every $r \in[k-1], W^{r}$ disconnects $W=W^{0}$ and $W^{k}$ in $G$ and hence, as $G$ is $p$-connected, we get $\left|W^{r}\right| \geq p$. Due to Eq. (70), we can now derive $\mathrm{d}_{G^{k}, \pi}(i) \geq(k-1) p$, as desired.

Proof of Theorem 66: Let $n=|V(G)|$. Assume that $\pi_{1} \cdots \pi_{n}$ is an ordering of $V(G)$ such that $\operatorname{dist}_{G}\left(\pi_{i}, \pi_{i+1}\right) \leq k$ and $G\left[\pi_{i}, \ldots, \pi_{n}\right]$ is connected for each $i \in[n-k]$. From $\operatorname{dist}_{G}\left(\pi_{i}, \pi_{i+1}\right) \leq k$ for all $i \in[n-k]$ and that $G\left[\pi_{n-k}, \ldots, \pi_{n}\right]$ is connected, we see that $\pi_{1} \cdots \pi_{n}$ is a Hamiltonian vertex ordering and $\left\{\pi_{n-k}, \ldots, \pi_{n}\right\}$ is a clique of $G^{k}$. Take $i \in[n-k]$. It remains to show that $\mid N_{G^{k}}\left(\pi_{i}\right) \cap$ $\left\{\pi_{i+1}, \ldots, \pi_{n}\right\} \mid \geq k$. But this follows from the fact that $G\left[\pi_{i}, \ldots, \pi_{n}\right]$ is a connected graph with at least $k+1$ vertices.

The latter part of our proof for Theorem 68 is analogous to the corresponding part of our proof for Theorem 64

Proof of Theorem 68: Let $P$ be an interval order such that $G$ is its cocomparability graph. Let $\pi=$ $\pi_{1} \cdots \pi_{n}$ be an ordinary ordering of $P$. Note that $n=|V(G)| \geq k p+1 \geq 3$. By Theorem 63, $\pi$ is a 2 -good vertex ordering of $G$ and hence a Hamiltonian vertex ordering of $G^{k}$. What remains to show is that $\mathrm{d}_{G^{k}, \pi}(i) \geq \min \{k p, n-i\}$ for all $i \in[n]$. It is clear that

$$
\mathrm{d}_{G^{k}, \pi}(i)=\cup_{q=1}^{k}\left|S_{q}\right|
$$

where $S_{q}=\left\{\pi_{t}: n \geq t>i, \operatorname{dist}_{G}\left(\pi_{i}, \pi_{t}\right)=q\right\}$ for $q \in[k]$. Also note that $G\left[\pi_{i}, \ldots, \pi_{n}\right]$ is a $k$ connected graph whenever $n-i \geq k$ as we obtain it from the $k$-connected interval graph $G$ by repeatedly removing bottom elements of the associated interval orders.

In view of Lemma 122, $G\left[\pi_{i}, \ldots, \pi_{n}\right]$ is connected for every $i \in[n]$. So, $\max _{j: i \leq j \leq n} \operatorname{dist}_{G}\left(\pi_{i}, \pi_{j}\right)$ is a finite number, which we denote by $D$. If $D \leq k, \mathrm{~d}_{G^{k}, \pi}(i)=\cup_{q=1}^{D}\left|S_{q}\right|=n-i$ and thus we are done. 
If $D>k, S_{1}, \ldots, S_{k}$ are all separating sets of $G\left[\pi_{i}, \ldots, \pi_{n}\right]$ and hence all have size at least $k$. It thus comes from Eq. (71) that $\mathrm{d}_{G^{k}, \pi}(i) \geq k p$, which ends the proof.

Proof of Theorem 70; If $\mathcal{H}(G)>0$, then $\beta=0$ and $G$ is traceable and hence $\operatorname{trn}(G)=n-1$. This means that both equalities in the claim hold true.

We continue to deal with the case of $\mathcal{H}(G) \leq 0$. Notice that we now have $\beta=\max \{0,1-\mathcal{H}(G)\}=$ $1-\mathcal{H}(G)$. Utilizing Eq. [2] in addition, we arrive at $\operatorname{trn}(G) \geq n-1+(\pi(G)-1)=n-2+(2-\mathcal{H}(G))=$ $n-1+\beta$. It remains to prove $\operatorname{trn}(G) \leq n-1+2 \beta$.

Let $\mathcal{I}$ be any interval representation of $G$. Assume that $\pi$ is an output of $\operatorname{NP}(G, \mathcal{I}, a)$ where $a$ is a leftmost vertex of $G$ with respect to $\mathcal{I}$. We claim that $\operatorname{dist}_{G}\left(\pi_{i}, \pi_{i+1}\right) \leq 3$ for each $i \in[n-1]$. This is guaranteed by Theorem 61 and the fact that $\pi$ is a path ordering of $G$. But let us present a more direct argument for this below. If the claim were not true, there is some $i \in[n-1]$ such that $\operatorname{dist}_{G}\left(\pi_{i}, \pi_{i+1}\right)>$ 3. Take any shortest path in $G$ from $\pi_{i}$ to $\pi_{i+1}$, say $P=\left(\tau_{1}, \ldots, \tau_{j}\right)$, where $j \geq 5, \tau_{1}=\pi_{i}$ and $\tau_{j}=\pi_{i+1}$. By Lemma 103 it holds $\mathcal{I}\left(\pi_{i}\right)<\mathcal{I}\left(\pi_{i+1}\right)$. Since $P$ is a shortest path, we further derive $\mathcal{I}\left(\pi_{i}\right)<\mathcal{I}\left(\pi_{k}\right)<\mathcal{I}\left(\pi_{i+1}\right)$ where $\pi_{k}=\tau_{3}$. By Lemma 103 again, this leads to $i<k<i+1$, which is impossible.

By the equivalence of a) and d) in Theorem 35, we get $\mathcal{H}_{G}(\pi)=\mathcal{H}(G)$ and $\mid\left\{i: \pi_{i} \pi_{i+1} \notin E(G), i \in\right.$ $[n-1]\} \mid=1-\mathcal{H}(G)=\beta$. This allows us to obtain $\operatorname{trn}(G) \leq \operatorname{dist}_{G}(\pi) \leq 3 \beta+(n-1-\beta)=n-1+2 \beta$, completing the proof.

\section{Acknowledgements}

We thank Andreas Dress, Ziqing Xiang, Xuding Zhu and Yinfeng Zhu for their help and encouragement. 


\section{Bibliography}

[1] A. Abueida and R. Srithran. Cycle extendability and Hamiltonian cycles in chordal graph classes. SIAM Journal on Discrete Mathematics, 20:669-681, 2006. ISSN 0895-4801. doi: 10.1137/S0895480104441267. URL http://dx.doi.org/10.1137/S0895480104441267

[2] M. Albert, R. Aldred, and D. Holton. On $3^{*}$-connected graphs. Australasian Journal of Combinatorics, 24: 193-208, 2001.

[3] S. Arikati and C. Pandu Rangan. Linear algorithm for optimal path cover problem on interval graphs. Information Processing Letters, 35:149-153, 1990. ISSN 0020-0190. doi: http://dx.doi.org/10. 1016/0020-0190(90)90064-5. URL http://www.sciencedirect.com/science/article/pii/ 0020019090900645

[4] K. Asdre and S. Nikolopoulos. The 1-fixed-endpoint path cover problem is polynomial on interval graphs. Algorithmica, 58:679-710, 2010. doi: 10.1007/s00453-009-9292-5. URL http://dx.doi.org/10. $1007 / \mathrm{s} 00453-009-9292-5$

[5] M. Baker and S. Norine. Riemann-Roch and Abel-Jacobi theory on a finite graph. Advances in Mathematics, 215:766-788, 2007. ISSN 0001-8708. doi: http://dx.doi.org/10.1016/j.aim.2007.04.012. URL http:// WwW.sciencedirect.com/science/article/pii/s0001870807001454

[6] J. Barnett. Early writings on graph theory: Hamiltonian circuits and the Icosian game. In B. Hopkins, editor, Resources for Teaching Discrete Mathematics: Classroom Projects, History Modules and Articles, pages 217223, Washington, D.C., 2009. Mathematical Association of America.

[7] D. Bauer, H. Broersma, J. van den Heuvel, N. Kahl, and E. Schmeichel. Toughness and vertex degrees. Journal of Graph Theory, 72:209-219, 2013. ISSN 1097-0118. doi: 10.1002/jgt.21639. URL http://dx.doi. org/10.1002/jgt.21639

[8] A. A. Bertossi. Finding Hamiltonian circuits in proper interval graphs. Information Processing Letters, 17:97101, 1983. ISSN 0020-0190. doi: http://dx.doi.org/10.1016/0020-0190(83)90078-9. URL http://www . sciencedirect.com/science/article/pii/0020019083900789

[9] J. Bondy and U. Murty. Graph Theory. Springer, 2008.

[10] H. Broersma, Z. Ryjáček, and P. Vrána. How many conjectures can you stand? A survey. Graphs and Combinatorics, 28:57-75, 2012. ISSN 0911-0119. doi: 10.1007/s00373-011-1090-6. URL http://dx . doi.org/10.1007/s00373-011-1090-6

[11] H. Broersma, J. Fiala, P. Golovach, T. Kaiser, D. Paulusma, and A. Proskurowski. Linear-time algorithms for scattering number and Hamiltonian-connectivity of interval graphs. In A. Brandstädt, K. Jansen, and R. Reischuk, editors, Proceedings of the 39th International Workshop on Graph-Theoretic Concepts in Computer Science (WG 2013), volume 8165 of Lecture Notes in Computer Science, pages 127-138. Springer Berlin Heidelberg, Luebeck, Germany, 2013. URL http://dx.doi.org/10.1007/978-3-642-45043-3_12

[12] F. Bullock, P. Dankelmann, M. Frick, M. A. Henning, O. R. Oellermann, and S. A. van Aardt. Hamiltonicity of $k$-traceable graphs. Electronic Journal of Combinatorics, 18:Paper \#P63, 13 p., 2011. URL http: //www . combinatorics.org/Volume_18/Abstracts/v18i1p63.html

[13] J. Chang, C. Ho, and M. Ko. Powers of asteroidal triple-free graphs with applications. Ars Combinatoria, 67: 161-173, 2003. 
[14] G. Chartrand, S. Kapoor, and D. R. Lick. n-Hamiltonian graphs. Journal of Combinatorial Theory, 9:308312, 1970. ISSN 0021-9800. doi: http://dx.doi.org/10.1016/S0021-9800(70)80069-2. URL http://www . sciencedirect.com/science/article/pii/s0021980070800692

[15] C. Chen and C.-C. Chang. Connected proper interval graphs and the guard problem in spiral polygons. In M. Deza, R. Euler, and I. Manoussakis, editors, Combinatorics and Computer Science, volume 1120 of Lecture Notes in Computer Science, pages 39-47. Springer Berlin Heidelberg, Brest, France, 1996. doi: 10.1007/ 3-540-61576-8_71.

[16] C. Chen and N. Quimpo. On strongly hamiltonian abelian group graphs. In K. L. McAvaney, editor, Combinatorial Mathematics VIII, Proceedings of the Eighth Australian Conference held at Deakin University, Geelong, August 25-29, 1980, volume 884 of Lecture Notes in Mathematics, pages 23-34. Springer Berlin Heidelberg, 1981. ISBN 978-3-540-10883-2. doi: 10.1007/BFb0091805. URL http://dx.doi.org/10.1007/ BFb0091805.

[17] C. Chen, C.-C. Chang, and G. J. Chang. Proper interval graphs and the guard problem. Discrete Mathematics, 170:223-230, 1997. ISSN 0012-365X. doi: http://dx.doi.org/10.1016/S0012-365X(96)00307-X. URL http: //www.sciencedirect.com/science/article/pii/s0012365X9600307X

[18] G. Chen, R. J. Gould, and F. Pfender. New conditions for $k$-ordered Hamiltonian graphs. Ars Combinatoria, 70, 2004.

[19] G. Chen, R. J. Faudree, R. J. Gould, and M. S. Jacobson. Cycle extendability of hamiltonian interval graphs. SIAM Journal on Discrete Mathematics, 20(3):682-689, 2006. doi: 10.1137/S0895480104441450. URL http://dx.doi.org/10.1137/s0895480104441450

[20] X. Chen, M. Szegedy, and L. Wang. Optimally balanced forward degree sequence. In L. Wang, editor, Computing and Combinatorics, volume 3595 of Lecture Notes in Computer Science, pages 680-689. Springer Berlin Heidelberg, 2005. ISBN 978-3-540-28061-3. doi: 10.1007/11533719_69. URL http://dx.doi. org/10.1007/11533719_69.

[21] Y. Chen, Z.-H. Chen, H.-J. Lai, P. Li, and E. Wei. On spanning disjoint paths in line graphs. Graphs and Combinatorics, 29(6):1721-1731, 2013. ISSN 0911-0119. doi: 10.1007/s00373-012-1237-0. URL http: //dx.doi.org/10.1007/s00373-012-1237-0

[22] M. Chrobak and D. Eppstein. Planar orientations with low out-degree and compaction of adjacency matrices. Theoretical Computer Science, 86(2):243-266, 1991. ISSN 0304-3975. doi: http://dx.doi.org/10. 1016/0304-3975(91)90020-3. URL http://www.sciencedirect.com/science/article/pii/ 0304397591900203

[23] V. Chvátal. Tough graphs and Hamiltonian circuits. Discrete Mathematics, 306(10-11):910-917, 2006. ISSN 0012-365X. doi: http://dx.doi.org/10.1016/j.disc.2006.03.011. URL http://www . sciencedirect. com/science/article/pii/s0012365X06001397. 35th Special Anniversary Issue.

[24] V. Chvátal and P. Erdôs. A note on Hamiltonian circuits. Discrete Mathematics, 2(2):111-113, 1972. ISSN 0012-365X. doi: http://dx.doi.org/10.1016/0012-365X(72)90079-9. URL http://www . sciencedirect.com/science/article/pii/0012365X72900799

[25] W. Cook. In Pursuit of the Traveling Salesman: Mathematics at the Limits of Computation. Princeton University Press, 2012. 
[26] D. G. Corneil, E. Kőhler, S. Olariu, and L. Stewart. Linear orderings of subfamilies of AT-free graphs. SIAM Journal on Discrete Mathematics, 20(1):105-118, 2006. doi: 10.1137/S0895480104445307. URL http: //dx.doi.org/10.1137/S0895480104445307

[27] D. G. Corneil, B. Dalton, and M. Habib. LDFS-based certifying algorithm for the minimum path cover problem on cocomparability graphs. SIAM Journal on Computing, 42(3):792-807, 2013. doi: 10.1137/11083856X. URL http://dx.doi.org/10.1137/11083856x.

[28] H. Coxeter. My graph. Proceedings of the London Mathematical Society, Third Series, 46:117-136, 1983.

[29] P. Damaschke. Paths in interval graphs and circular arc graphs. Discrete Mathematics, 112(1):49-64, 1993. ISSN 0012-365X. doi: http://dx.doi.org/10.1016/0012-365X(93)90223-G. URL http://www . sciencedirect.com/science/article/pii/0012365X9390223G

[30] P. Damaschke, J. S. Deogun, D. Kratsch, and G. Steiner. Finding Hamiltonian paths in cocomparability graphs using the bump number algorithm. Order, 8(4):383-391, 1991. ISSN 0167-8094. doi: 10.1007/BF00571188. URL http://dx.doi.org/10.1007/BF00571188.

[31] A. M. Dean, C. J. Knickerbocker, P. F. Lock, and M. Sheard. A survey of graphs Hamiltonian-connected from a vertex. In Y. Alavi, G. Chartrand, O. Oellermann, and A. Schwenk, editors, Graph Theory, Combinatorics, and Applications, Vol. 1, Proceedings of the Sixth Quadrennial International Conference on the Theory and Applications of Graphs held at Western Michigan University, Kalamazoo, Michigan, May 30-June 3, 1988, pages 297-313. Wiley, New York, 1991.

[32] J. S. Deogun, D. Kratsch, and G. Steiner. 1-Tough cocomparability graphs are Hamiltonian. Discrete Mathematics, 170(1-3):99-106, 1997. ISSN 0012-365X. doi: http://dx.doi.org/10.1016/0012-365X(95)00359-5. URL http://www.sciencedirect.com/science/article/pii/0012365X95003595

[33] G. Dirac. On rigid circuit graphs. Abhandlungen Aus Dem Mathematischen Seminar Der Universitat Hamburg, 25:71-76, 1961.

[34] P. Erdős and A. Hajnal. On chromatic number of graphs and set-systems. Acta Mathematica Academiae Scientiarum Hungarica, 17(1-2):61-99, 1966. ISSN 0001-5954. doi: 10.1007/BF02020444. URL http: //dx.doi.org/10.1007/BF02020444

[35] M. Farber. Characterizations of strongly chordal graphs. Discrete Mathematics, 43(2-3):173-189, 1983. ISSN 0012-365X. doi: http://dx.doi.org/10.1016/0012-365X(83)90154-1. URL http://www . sciencedirect.com/science/article/pii/0012365X83901541

[36] D. Franzblau and A. Raychaudhuri. Optimal Hamiltonian completions and path covers for trees, and a reduction to maximum flow. ANZIAM Journal, 44:193-204, 2002.

[37] T. Gaudin, J. Herz, and P. Rossi. Solution du probl'e me no. 29. Revue Française d'Informatique et de Recherche Opérationnelle, 8:214-218, 1964.

[38] V. Giakoumakis, F. Roussel, and H. Thuillier. Scattering number and modular decomposition. Discrete Mathematics, 165-166:321-342, 1997. ISSN 0012-365X. doi: http://dx.doi.org/10.1016/S0012-365X(96)00180-X. URL http://www.sciencedirect.com/science/article/pii/s0012365X9600180X

[39] V. Giakoumakis, F. Roussel, and H. Thuillier. On $P_{4}$-tidy graphs. Discrete Mathematics \& Theoretical Computer Science, 1(1):17-41, 1997. URL http://dmtcs.loria.fr/volumes/abstracts/ dm010102.abs.html 
[40] M. C. Golumbic. Algorithmic Graph Theory and Perfect Graphs, volume 57 of Annals of Discrete Mathematics. Elsevier Science B.V., Amsterdam, second edition, 2004. ISBN 0-444-51530-5. With a foreword by Claude Berge.

[41] R. Gould and T. Whalen. Subdivision extendibility. Graphs and Combinatorics, 23(2):165-182, 2007. ISSN 0911-0119. doi: 10.1007/s00373-006-0665-0. URL http://dx.doi.org/10.1007/ s00373-006-0665-0

[42] R. J. Gould. A look at cycles containing specified elements of a graph. Discrete Mathematics, 309(21): 6299-6311, 2009. ISSN 0012-365X. doi: http://dx.doi.org/10.1016/j.disc.2008.04.017. URL http://www . sciencedirect.com/science/article/pii/s0012365X08002318

[43] F. Harary and J. P. Hayes. Edge fault tolerance in graphs. Networks, 23(2):135-142, 1993. ISSN 1097-0037. doi: 10.1002/net.3230230207. URL http://dx.doi.org/10.1002/net.3230230207

[44] G. R. Hendry. Extending cycles in graphs. Discrete Mathematics, 85(1):59-72, $1990 . \quad$ ISSN 0012365X. doi: http://dx.doi.org/10.1016/0012-365X(90)90163-C. URL http://www . sciencedirect. com/science/article/pii/0012365X9090163C

[45] C.-W. Ho and R. Lee. Counting clique trees and computing perfect elimination schemes in parallel. Information Processing Letters, 31(2):61-68, 1989. ISSN 0020-0190. doi: http://dx.doi.org/10. 1016/0020-0190(89)90070-7. URL http://www.sciencedirect.com/science/article/pii/ 0020019089900707

[46] T.-Y. Ho, C.-K. Lin, J. J. Tan, D. F. Hsu, and L.-H. Hsu. On the extremal number of edges in Hamiltonian connected graphs. Applied Mathematics Letters, 23(1):26-29, 2010. ISSN 0893-9659. doi: http://dx. doi.org/10.1016/j.aml.2009.03.025. URL http://www.sciencedirect.com/science/article/ pii/S0893965909002882

[47] W. Hochstättler and G. Tinhofer. Hamiltonicity in graphs with few $P_{4}{ }^{\prime}$ s. Computing, 54(3):213-225, 1995. ISSN 0010-485X. doi: 10.1007/BF02253613. URL http://dx.doi.org/10.1007/BF02253613

[48] S.-Y. Hsieh, G.-H. Chen, and C.-W. Ho. Fault-free Hamiltonian cycles in faulty arrangement graphs. IEEE Transactions on Parallel and Distributed Systems, 10(3):223-237, Mar 1999. ISSN 1045-9219. doi: 10.1109/71.755822. URL http://ieeexplore.ieee.org/xpl/articleDetails.jsp? arnumber $=755822$

[49] D. F. Hsu. On container width and length in graphs, groups, and networks. IEICE Transactions Fundamentals of Electronics, Communications and Computer Sciences, E77-A(4):668-680, 1994. URL http://i-scover.ieice.org/iscover/page/ ARTICLE-666DDD00-8B85-A795-5C47-7E7600C7A02B.

[50] L.-H. Hsu and C.-K. Lin. Graph Theory and Interconnection Networks. CRC Press, Boca Raton, 2009.

[51] Z. Hu, F. Tian, and B. Wei. Hamilton connectivity of line graphs and claw-free graphs. Journal of Graph Theory, 50(2):130-141, 2005. ISSN 1097-0118. doi: 10.1002/jgt.20099. URL http://dx.doi.org/ $10.1002 / j g t .20099$

[52] P.-Y. Huang and L.-H. Hsu. The spanning connectivity of line graphs. Applied Mathematics Letters, 24(9): 1614-1617, 2011. ISSN 0893-9659. doi: http://dx.doi.org/10.1016/j.aml.2011.04.013. URL/http://www . sciencedirect.com/science/article/pii/s0893965911001741 
[53] W.-T. Huang, Y.-C. Chuang, J. Tan, and L.-H. Hsu. Fault-free Hamiltonian cycle in faulty Möbius cubes. Computación y Systemas, 4:106-114, 2000.

[54] R.-W. Hung and M.-S. Chang. Linear-time certifying algorithms for the path cover and Hamiltonian cycle problems on interval graphs. Applied Mathematics Letters, 24(5):648-652, 2011. ISSN 08939659. doi: http://dx.doi.org/10.1016/j.aml.2010.11.030. URL http://www.sciencedirect.com/ science/article/pii/s0893965910004490

[55] L. Ibarra. The clique-separator graph for chordal graphs. Discrete Applied Mathematics, 157(8):17371749, 2009. ISSN 0166-218X. doi: http://dx.doi.org/10.1016/j.dam.2009.02.006. URL http://www . sciencedirect.com/science/article/pii/s0166218X09000390

[56] K. Ioannidou, G. B. Mertzios, and S. D. Nikolopoulos. The longest path problem has a polynomial solution on interval graphs. Algorithmica, 61(2):320-341, 2011. ISSN 0178-4617. doi: 10.1007/s00453-010-9411-3. URL http://dx.doi.org/10.1007/s00453-010-9411-3.

[57] G. Isaak. Powers of Hamiltonian paths in interval graphs. Journal of Graph Theory, 28(1):31-38, 1998. ISSN 1097-0118. doi: 10.1002/(SICI)1097-0118(199805)28:1〈31::AID-JGT3〉3.0.CO;2-G. URL http: //dx.doi.org/10.1002/(SICI) 1097-0118(199805) 28:1<31::AID-JGT3>3.0.CO;2-G

[58] J. Jamrozik, R. Kalinowski, and Z. Skupień. A catalogue of small maximal nonhamiltonian graphs. Discrete Mathematics, 39(2):229-234, 1982. ISSN 0012-365X. doi: http://dx.doi.org/10.1016/0012-365X(82)90145-5. URL http://www.sciencedirect.com/science/article/pii/0012365X82901455

[59] S. Jo, J.-H. Park, and K.-Y. Chwa. Paired many-to-many disjoint path covers in faulty hypercubes. Theoretical Computer Science, 513:1-24, 2013. ISSN 0304-3975. doi: http://dx.doi.org/10.1016/j.tcs.2013.10.008. URL http://www.sciencedirect.com/science/article/pii/s0304397513007603.

[60] H. Jung. On a class of posets and the corresponding comparability graphs. Journal of Combinatorial Theory, Series B, 24(2):125-133, 1978. ISSN 0095-8956. doi: http://dx.doi.org/10.1016/0095-8956(78)90013-8. URL http://www.sciencedirect.com/science/article/pii/0095895678900138

[61] J. Karaganis. On the cube of a graph. Canadian Mathematical Bulletin, 11:295-296, 1968. URL http: //cms.math.ca/10.4153/CMB-1968-037-0

[62] J. M. Keil. Finding Hamiltonian circuits in interval graphs. Information Processing Letters, 20(4):201206, 1985. ISSN 0020-0190. doi: http://dx.doi.org/10.1016/0020-0190(85)90050-X. URL http://www . sciencedirect.com/science/article/pii/002001908590050X

[63] H. A. Kierstead, G. N. Sárközy, and S. M. Selkow. On k-ordered Hamiltonian graphs. Journal of Graph Theory, 32(1):17-25, 1999. ISSN 1097-0118. doi: 10.1002/(SICI)1097-0118(199909)32:1<17:: AID-JGT2)3.0.CO;2-G. URL http: // dx.doi.org/10.1002/(SICI) 1097-0118(199909) 32: $1<17:: \mathrm{AID}-\mathrm{JGT} 2>3.0 . \mathrm{CO} ; 2-\mathrm{G}$

[64] L. M. Kirousis and D. M. Thilikos. The linkage of a graph. SIAM Journal on Computing, 25(3):626-647, 1996. doi: 10.1137/S0097539793255709. URLhttp://dx.doi.org/10.1137/S0097539793255709.

[65] D. Kratsch and L. Stewart. Domination on cocomparability graphs. SIAM Journal on Discrete Mathematics, 6(3):400-417, 1993. doi: 10.1137/0406032. URL http://dx.doi.org/10.1137/0406032 
[66] D. Kratsch, T. Kloks, and H. Müller. Computing the toughness and the scattering number for interval and other graphs. Technical Report IRISA-PI-94-806, INRIA Rennes, 1994. URL http://hdl.handle.net/ $10068 / 56937$

[67] D. Kratsch, J. Lehel, and H. Müller. Toughness, hamiltonicity and split graphs. Discrete Mathematics, 150 (1-3):231-245, 1996. ISSN 0012-365X. doi: http://dx.doi.org/10.1016/0012-365X(95)00190-8. URL http: //www.sciencedirect.com/science/article/pii/0012365X95001908.

[68] H.-J. Lai and Y. Shao. On s-Hamiltonian line graphs. Journal of Graph Theory, 74(3):344-358, 2013. ISSN 1097-0118. doi: 10.1002/jgt.21713. URL http://dx.doi.org/10.1002/jgt.21713

[69] H.-J. Lai, Y. Liang, and Y. Shao. On $s$-Hamiltonian-connected line graphs. Discrete Mathematics, 308(18): 4293-4297, 2008. ISSN 0012-365X. doi: http://dx.doi.org/10.1016/j.disc.2007.07.120. URL http: //www . sciencedirect.com/science/article/pii/s0012365X0700670X

[70] D. Larman and P. Mani. On the existence of certain configurations within graphs and the 1-skeleons of polytopes. Proceedings of the London Mathematical Society, 20:144-160, 1974.

[71] J.-H. Lee and J.-H. Park. General-demand disjoint path covers in a graph with faulty elements. International Journal of Computer Mathematics, 89:606-617, 2012.

[72] J. Lehel. The path partition of cocomparability graphs. 1991.

[73] P. Li and Y. Wu. Maximal neighborhood search and rigid interval graphs. Journal of Graph Algorithms and Applications, 17(3):245-264, 2013. doi: 10.7155/jgaa.00293. URL http://jgaa.info/getPaper? id $=293$

[74] P. Li and Y. Wu. A linear time algorithm for the 1-fixed-endpoint path cover problem on interval graphs. submitted, 2014. URL http://math.s jtu.edu.cn/faculty/ykwu/data/Paper/20150909. $\mathrm{pdf}$

[75] D. Lick and A. White. k-Degenerate graphs. Canadian Journal of Mathematics, 22:1082-1096, 1970.

[76] C.-K. Lin, H.-M. Huang, and L.-H. Hsu. On the spanning connectivity of graphs. Discrete Mathematics, 307(2):285-289, 2007. ISSN 0012-365X. doi: http://dx.doi.org/10.1016/j.disc.2006.06.021. URL http: //wWw.sciencedirect.com/science/article/pii/s0012365X06004845

[77] C.-K. Lin, H.-M. Huang, J. J. Tan, and L.-H. Hsu. On spanning connected graphs. Discrete Mathematics, 308(7):1330-1333, 2008. ISSN 0012-365X. doi: http://dx.doi.org/10.1016/j.disc.2007.03.072. URL http: //www.sciencedirect.com/science/article/pii/s0012365x07002002

[78] C.-K. Lin, J. J. Tan, D. F. Hsu, and L.-H. Hsu. On the spanning fan-connectivity of graphs. Discrete Applied Mathematics, 157(7):1342-1348, 2009. ISSN 0166-218X. doi: http://dx.doi.org/10.1016/j.dam.2008.11.014. URL http://www.sciencedirect.com/science/article/pii/s0166218X08005064

[79] W. Mader. Homomorphieeigenschaften und mittlere kantendichte von graphen. Mathematische Annalen, 174: 265-268, 1967.

[80] G. K. Manacher, T. A. Mankus, and C. J. Smith. An optimum $\Theta(n \log n)$ algorithm for finding a canonical Hamiltonian path and a canonical hamiltonian circuit in a set of intervals. Information Processing Letters, 35 (4):205-211, 1990. ISSN 0020-0190. doi: http://dx.doi.org/10.1016/0020-0190(90)90025-S. URL http: //wWw.sciencedirect.com/science/article/pii/002001909090025S. 
[81] M. M. Matthews and D. P. Sumner. Hamiltonian results in $K_{1,3}$-free graphs. Journal of Graph Theory, $8(1)$ : 139-146, 1984. ISSN 1097-0118. doi: 10.1002/jgt.3190080116. URL http://dx.doi .org/10.1002/ jgt. 3190080116 .

[82] G. B. Mertzios and D. G. Corneil. A simple polynomial algorithm for the longest path problem on cocomparability graphs. SIAM Journal on Discrete Mathematics, 26(3):940-963, 2012. doi: 10.1137/100793529. URL http://dx.doi.org/10.1137/100793529.

[83] Š. Miklavič and P. Šparl. Hamilton cycle and Hamilton path extendability of Cayley graphs on abelian groups. Journal of Graph Theory, 70(4):384-403, 2012. ISSN 1097-0118. doi: 10.1002/jgt.20621. URL http: //dx.doi.org/10.1002/jgt.20621

[84] J. Moon. On a problem of Ore. The Mathematical Gazette, 49:40-41, 1965.

[85] L. Ng and M. Schultz. k-Ordered Hamiltonian graphs. Journal of Graph Theory, 24(1):45-57, 1997. ISSN 1097-0118. doi: 10.1002/(SICI)1097-0118(199701)24:1〈45::AID-JGT6 3.0.CO;2-J. URL http://dx . doi.org/10.1002/(SICI) 1097-0118(199701) 24:1<45::AID-JGT6>3.0.CO;2-J

[86] D. J. Oberly and D. P. Sumner. Every connected, locally connected nontrivial graph with no induced claw is Hamiltonian. Journal of Graph Theory, 3(4):351-356, 1979. ISSN 1097-0118. doi: 10.1002/jgt.3190030405. URL http://dx.doi.org/10.1002/jgt.3190030405

[87] O. Ore. Hamiltonian connected graphs. Journal de Mathématiques Pures et Appliquées, Neuviéme Série, 42: 21-27, 1963.

[88] K. Ozeki and T. Yamashita. Hamiltonian cycles and dominating cycles passing through a linear forest. Discrete Mathematics, 309(6):1584-1592, 2009. ISSN 0012-365X. doi: http://dx.doi.org/10.1016/j.disc.2008.02.031. URL http://www.sciencedirect.com/science/article/pii/s0012365X08001404

[89] B. Panda and S. K. Das. A parallel algorithm for generating bicompatible elimination orderings of proper interval graphs. Information Processing Letters, 109(18):1041-1046, 2009. ISSN 0020-0190. doi: http://dx. doi.org/10.1016/j.ipl.2009.06.011. URL http://www.sciencedirect.com/science/article/ pii/S002001900900194X

[90] J.-H. Park and I. Ihm. Single-source three-disjoint path covers in cubes of connected graphs. Information Processing Letters, 113(14-16):527-532, 2013. ISSN 0020-0190. doi: http://dx.doi.org/ 10.1016/j.ipl.2013.04.012. URL http://www.sciencedirect.com/science/article/pii/ S0020019013001294

[91] J.-H. Park, H.-C. Kim, and H.-S. Lim. Many-to-many disjoint path covers in hypercube-like interconnection networks with faulty elements. IEEE Transactions on Parallel and Distributed Systems, 17(3):227-240, 2006.

[92] L. Pósa. On the circuits of finite graphs. Magyar Tud. Akad. Mat. Kutató Int. Közl., 8:355-361, 1964.

[93] A. Raychaudhuri. On powers of interval and unit interval graphs. Congressus Numerantium, 59:235-242, 1987.

[94] F. S. Roberts. Indifference graphs. In F. Harary, editor, Proof Techniques in Graph Theory, pages 139-146. Academic Press, 1969.

[95] N. Robertson and P. Seymour. Graph minors XIII: the disjoint paths problem. Journal of Combinatorial Theory, Series B, 63(1):65-110, 1995. ISSN 0095-8956. doi: http://dx.doi.org/10.1006/jctb.1995.1006. URL http://www.sciencedirect.com/science/article/pii/s0095895685710064. 
[96] E. Sabir and E. Vumar. Spanning connectivity of the power of a graph and Hamilton-connected index of a graph. Graphs and Combinatorics, 30(6):1551-1563, 2014. ISSN 0911-0119. doi: 10.1007/ s00373-013-1362-4. URL http://dx.doi.org/10.1007/s00373-013-1362-4

[97] V. Saenpholphat, F. Okamoto, and P. Zhang. Measures of traceability in graphs. Mathematica Bohemica, 131 (1):63-84, 2006. URL http://www.emis.ams.org/journals/MB/131.1/mb131_1_7.pdf

[98] A. Saito. Chvátal-Erdös Theorem: Old theorem with new aspects. In H. Ito, M. Kano, N. Katoh, and Y. Uno, editors, Computational Geometry and Graph Theory, volume 4535 of Lecture Notes in Computer Science, pages 191-200. Springer Berlin Heidelberg, 2008. ISBN 978-3-540-89549-7. doi: 10.1007/ 978-3-540-89550-3_21. URL http://dx.doi.org/10.1007/978-3-540-89550-3_21

[99] C. Savage. A survey of combinatorial Gray codes. SIAM Review, 39(4):605-629, 1997. doi: 10.1137/ S0036144595295272. URL http://dx.doi.org/10.1137/S0036144595295272

[100] M. Sekanina. On an ordering of the set of vertices of a connected graph. Publication of the Faculty of Sciences of the University of Brně, 412:137-142, 1960.

[101] W.-K. Shih, T. C. Chern, and W.-L. Hsu. An $O\left(n^{2} \log n\right)$ algorithm for the Hamiltonian cycle problem on circular-arc graphs. SIAM Journal on Computing, 21(6):1026-1046, 1992. doi: 10.1137/0221061. URL http://dx.doi.org/10.1137/0221061

[102] B. A. Sørensen and C. Thomassen. On $k$-rails in graphs. Journal of Combinatorial Theory, Series B, 17 (2):143-159, 1974. ISSN 0095-8956. doi: http://dx.doi.org/10.1016/0095-8956(74)90082-3. URL http: //wWw.sciencedirect.com/science/article/pii/0095895674900823.

[103] H. Su, Y.-K. Shih, and S.-S. Kao. On the 1-fault hamiltonicity for graphs satisfying Ore's theorem. Information Processing Letters, 112(21):839-843, 2012. ISSN 0020-0190. doi: http://dx.doi.org/ 10.1016/j.ipl.2012.07.014. URL http://www.sciencedirect.com/science/article/pii/ S0020019012002025

[104] C. Thomassen. Hypohamiltonian and hypotraceable graphs. Discrete Mathematics, 9(1):91-96, 1974. ISSN 0012-365X. doi: http://dx.doi.org/10.1016/0012-365X(74)90074-0. URL http://www . sciencedirect.com/science/article/pii/0012365X74900740

[105] G. Wiener and M. Araya. On planar hypohamiltonian graphs. Journal of Graph Theory, 67(1):55-68, 2011. ISSN 1097-0118. doi: 10.1002/jgt.20513. URL http://dx.doi.org/10.1002/jgt.20513

[106] D. R. Woodall. List colourings of graphs. In J. Hirschfeld, editor, Surveys in Combinatorics, 2001 (Sussex), volume 288 of London Mathematical Society Lecture Note Series, pages 269-301. Cambridge University Press, 2001 .

[107] Y. Wu, Z. Xiang, and Y. Zhu. Hamiltonian thickness and fault-tolerant spanning rooted path systems of graphs. 2015. 